\title{
REVIEW OF THE ANOTYLUS CIMICOIDES SPECIES GROUP (COLEOPTERA: STAPHYLINIDAE: OXYTELINAE)
}

\author{
GYÖRGY MAKRANCZY \\ Department of Zoology, Hungarian Natural History Museum \\ H-1088 Budapest, Baross utca 13, Hungary; E-mail: makranczy.gyorgy@nhmus.hu
}

\begin{abstract}
As a result of the moving of Oxytelopsis Fauvel, 1895 into the mega-diverse Anotylus Thomson, 1859 as a species group (A. cimicoides species group) a number of actions were necessitated (treating homonymies and ending changes inflicted by different gender of genera), forcing the reexamination of the involved taxa. This review is focused on the Oriental region and the transitional Himalayan area (including the southern provinces of China). 15 species are described as new to science: Anotylus coonoor sp. n. (India: Tamil Nadu), A. ganapati sp. n. (Nepal: Eastern Region), A. gunung sp. n. (Thailand: Yala prov.), A. hartmanni sp. n. (Nepal: Western Region), A. ijen sp. n. (Indonesia/Java: Banyuwangi), A. jambi sp. n. (Indonesia/Sumatra: Jambi), A. kabasi sp. n. (Indonesia/Sumatra: N. Sum.), A. linaxi sp. n. (China: Yunnan), A. riedeli sp. n. (Indonesia/Java: Bandung), A. rurukan sp. n. (Indonesia/ Sulawesi: Tomohon), A. schawalleri sp. n. (Malaysia/Borneo: Sabah), A. schillhammeri sp. n. (Myanmar: Chin State), A. schuelkei sp. n. (China: Yunnan), A. tanator sp. n. (Indonesia/ Sulawesi: Tanah Toraja), A. topali sp. n. (Vietnam: Ninh Binh). The following new synonymies are proposed: A. chinensis (Bernhauer, 1938) = Oxytelopsis shibatai Ito, 1987, syn. n., A. cimicoides (Fauvel, 1895) $=$ Oxytelopsis taiwana Ito, 1987, syn. n., A. malaisei (Scheerpeltz, $1965)=$ Oxytelopsis gardneri Paulian, 1940 (preoccupied), syn. n., A. pseudopsinus (Fauvel, $1895)=$ Oxytelopsis rufotestacea Cameron, 1925, syn. n., Oxytelopsis brevipennis Bernhauer, 1926 (preoccupied), syn. n., Oxytelopsis chapmani Cameron, 1934, syn. n., Oxytelopsis nigripennis Cameron, 1934, syn. n., Oxytelopsis cassagnaui Coiffait, 1982, syn. n., and lectotypes are designated for the following nominal species: Oxytelopsis andrewesi Cameron, 1930, Oxytelopsis anguliceps Cameron, 1934, Oxytelopsis apicipennis Fauvel, 1895, Oxytelopsis chinensis Bernhauer, 1938, Oxytelopsis cimicoides Fauvel, 1895, Oxytelopsis lucidula Cameron, 1936, Oxytelopsis nigricans Cameron, 1933 and Oxytelopsis pseudopsina Fauvel, 1895. A neotype for Oxytelopsis franzi Coiffait, 1982 is designated from Nepal (Central, prov. Bagmati), very near its original type locality. All the treated species are illustrated with colour habitus images and line drawings of their male genitalia and terminalia, spermathecae (where available) are illustrated for the new species.
\end{abstract}

Key words: Coleoptera, Staphylinidae, Oxytelinae, Anotylus, new species, new synonyms, lectotypes, neotype, brachypterous endemics, key, Palaearctic and Oriental regions.

\section{INTRODUCTION}

Oxytelopsis Fauvel, 1895 used to be one of the most easily recognizable genera of the subfamily Oxytelinae. Their appearance is characterized by a wide, flattened pronotum with crenuliform edge, strong body sculpture that readily absorbs soil particles (possible form of camouflage). HAMmond 
(1975) already indicated the possible necessity of absorbing Oxytelopsis into Anotylus Thomson, 1859 in a broader concept. The revision of generic limits (MaKranczy 2006) brought a change in the status of Oxytelopsis, it became part of Anotylus (as the A. cimicoides group). There are groups of species with rather similar external appearances known, primarily in the tropical regions (Africa, Central and South America) with not particularly close relatedness to the A. cimicoides group. However, the former Oxytelopsis was not consistently used at the beginning and applied to some Afrotropical taxa also (with somewhat similar external appearance). Even more, still undescribed African species have confusingly similar habitus, yet entirely different terminalia and genitalia (MAKRANCZY 2006) - these will be dealt with in a separate publication - as well as some from the Neotropics.

The present study was originally started to mend a rather awful situation of most nominal taxa either being based on a single female specimen - these are lacking reliable diagnostic characters - or their type materials lost, damaged or untraceable. This account focuses on the male characters, as it is a necessary first step towards the better knowledge of this group to pin down the identities of names. There is no practical way to assess the extremely great external morphological variability, so the most characteristic specimens with the best expression of their unique traits are illustrated - but often within the same series much less characteristic specimens are present. The female genitalia do not offer much in terms of diagnosis in this particular group. It must also be noted that female specimens in this work were most often identified by the association with males; by external features neither the poorly developed males nor the females can be determined with absolute certainty at this point. However, in order to give the best possible information about the taxa introduced here, the spermathecae of the new species are illustrated where available.

Some of the species have fully developed wings and consequently wide distribution range, but others possess wings reduced to various extent and shortened elytra. In the broader Himalaya area (plus Sri Lanka) such brachypterous species have very narrow distributional ranges (Hammond 1975) and surprisingly, there can be more than one species in the same area in relatively close proximity (A. montanus and A. ceylonicus in the highest parts of Sri Lanka, A. franzi and A. hartmanni in the central part of Nepal, A. bengalensis and A. jaljulensis along the far eastern borders of Nepal). In Southeast Asia the larger islands have their species more or less endemic to them, as opposed to, for example, the recently revised Paraploderus Herman, 1970 (MaKRanczy 2016), but in several instances (e.g. A. kabasi and A. nigricans) a dubiously identified specimen from a neighbouring island is known and this must be further investigated.

Constructing an identification key is problematic, because the variability in both size and development of features is so great that it leaves no ground for a practically usable key. Even in the terminalia and genitalia variation is con- 
siderable. Oxytelines frequently have sexual dimorphism that involves great size difference in either head (+pronotum) or the whole body between the sexes, but here enormous differences can be encountered within the same sex of a particular taxon, even though the group is not one where "macrocephaly" (large-headed "major" male usually with swollen temples) is expressed. Dirt (soil particles) adhering to body surface also makes external characters difficult to see and is very tricky to remove (see below).

\section{MATERIAL AND METHODS}

The study was started many years ago with a vague hope that the bad state of the taxonomy in this group can somehow be improved; after all these years the author is the most surprised at how much could actually be achieved. The initial approach, however, was very conservative, this is the reason why the present writer's usual "revisional" identification labels (with thick black frame) were not used (instead only "non-revisional" determination labels with thin black frame were placed on previously unidentified specimens). One important issue was how to handle P. M. Hammond's unpublished type designations (specimens were labelled in the early 1970s) that remain unpublished to the present day - since this is almost complete, it was decided that a duplicate labelling is to be avoided; instead the unpublished designations are hereby validated, where necessary.

Abbreviations of collections used in the present study are: $\mathrm{AMNH}=$ American $\mathrm{Mu}-$ seum of Natural History, New York, USA (L. H. Herman); ANIC = Australian National Insect Collection, ACT, Australia (S. A. Slipinski, C: Lemann); BMNH = The Natural History Museum, London, UK (R. G. Booth); CNCI = Canadian National Collection of Insects, Ottawa, Canada (A. Davies); FMNH = Field Museum of Natural History, Chicago, USA (J. H. Boone); HNHM = Hungarian Natural History Museum, Budapest, Hungary; ISNB = Institut Royal des Sciences Naturelles de Belgique, Bruxelles, Belgium (Y. Gérard); NSMT = Department of Zoology, National Museum of Nature and Science, Tsukuba, Ibaraki, Japan (S. Nomura); IZAS = Institute of Zoology, Chinese Academy of Sciences, Beijing, China (L. Lü); MBBJ = Museum Zoologicum Bogoriense, Cibinong Science Center, Bogor, Indonesia; MHNG = Muséum d'Histoire Naturelle, Genève, Switzerland (G. Cuccodoro); MNHP = Muséum National d'Histoire Naturelle, Paris, France (A. Taghavian); MZLU = Museum of Zoology, Lund University, Lund, Sweden (C. Fägerström); NHMW = Naturhistorisches Museum Wien, Austria (H. Schillhammer); NHRS = Naturhistoriska Riksmuseet, Stockholm, Sweden (B. Viklund, N. Apelqvist); NIBR = National Institute of Biological Resources, Incheon, Korea (M.-J. Jeon); NMPC = National Museum (Natural History), Prague, Czech Republic (J. Hájek); RMNH = Nationaal Natuurhistorische Museum ("Naturalis") [formerly Rijksmuseum van Natuurlijke Historie], Leiden, The Netherlands (H. Huijbregts); SMNK = Staatliches Museum für Naturkunde, Karlsruhe, Germany (A. Riedel); SMNS = Staatliches Museum für Naturkunde, Stuttgart, Germany (W. Schawaller); NKME = Naturkundemuseum Erfurt, Erfurt, Germany (M. Hartmann); ZFMK = Zoologisches Forschungsmuseum "Alexander Koenig", Bonn, Germany (D. Ahrens); ZMHB = ZMHB = Museum für Naturkunde, Leibniz Institute for Evolution and Biodiversity Science, Berlin, Germany (J. Willers, J. Frisch); ZMUC = Zoological Museum, University of Copenhagen, Denmark (A. Solodovnikov); coll. Assing = private collection of Volker Assing, Hannover, Germany; coll. Kleeberg = private collection of Andreas Kleeberg, Berlin, Germany; coll. Schülke = private collection of Michael Schülke, Berlin, Germany, property of ZMHB; coll. 
Ryvkin = private collection of Alexandr Ryvkin, Moscow, Russia; coll. Shavrin = private collection of Alexey Shavrin, Daugavpils, Latvia.

For descriptions and measurements a Leica MZ 12.5 stereoscopic microscope was used. For the line drawings permanent preparations were made in Euparal mounting medium on plastic cards pinned with the specimens. The genital preparation techniques are detailed in Makranczy (2006). Drawing was done with a Jenalab (Carl Zeiss, Jena) compound microscope and attached drawing tube (camera lucida). For the colour habitus photographs a Nikon D4 camera with Mitutoyo PlanApo $5 \times$ or $10 \times$ ELDW lens was used and layers montaged with ZereneStacker. In the illustration of the genitalia and terminalia a basic protocol established in MAKRANCZY (2011) is followed: aedeagi and the most diagnostic parts of the terminalia are shown in full detail, while only outline sketches of some of the other important abdominal plates are provided with emphasis on the diagnostic details.

Measurements are defined as follows: $\mathrm{HW}=$ head width with eyes; TW = head width at temples; $\mathrm{PW}=$ maximum width of pronotum; $\mathrm{EW}=$ maximum width of elytra; $\mathrm{AW}=$ maximum width of abdomen; $\mathrm{HL}=$ head length at the middle-line from front margin of clypeus to the beginning of neck; $\mathrm{EL}=$ eye length; $\mathrm{TL}=$ length of temple to the side border of neck; PL = length of pronotum at the middle-line; SC = length of elytra from hind apex of scutellum; $\mathrm{FB}$ = forebody length (combined length of head, pronotum and elytra); $\mathrm{BL}=$ approximate body length. All were measured from dorsal view.

The label data when reproduced literally are between " ", the symbol " $\backslash$ " is a separator between each individual label, while ";" means line breaks. Text within square brackets [ ] is explanatory and was not included in the original labels. The label data are often supplemented from secondary sources (internet, collection lists, other articles) as well as information received directly from collectors. Geographical coordinates from Google Earth are in square brackets to distinguish them from primary data. As there was no GPS at the time of most of the collecting data included here, such values are to be considered "a best possible approximation".

\section{TAXONOMY}

Inside a genus with such immense species number some subdivision is necessary. However, creation of a stable system is almost impossible before the diversity of the genus is more properly mapped - this remains a dream for the time being. Therefore, it must be accepted that any division implemented is at best temporary and subject to further changes. P. M. Hammond started to create a species group system, but this work has remained incomplete and for the most part, unpublished. Apart from one species group he revised (HAMMOND 1976b) and several others hinted in various works (e.g. HAMmOND 1975, Hammond 1976a, Hammond et al. 1979) his system is accessible only by the current arrangement and labelling of Anotylus specimens in the respective boxes of BMNH. However, as there is rarely any explanation of the character states used to delimit the groups (probably still somewhat inconsistent), this is of little practical use for a scholar today.

At present the best (and perhaps only) option is to go along with Hammond's unfinished system and refine it later as necessary. Some groups are already clear, named and possible to define - taxonomic treatments of these 
may reduce the workload with the more problematic assemblages to a manageable amount. Fortunately, the Anotylus cimicoides group is one of those that stand reasonably well; the most striking feature of the group is an enlarged paraproct of tergites IX in the males - the expression of this trait, however, varies between species. The aedeagi have rather broad and parallel-sided parameres, although this character state may not be unique to this group and also shows a degree of variation among the species.

Key to species of the Anotylus cimicoides group

1 Large species (PL above $0.75 \mathrm{~mm}$, PW usually $>1.15 \mathrm{~mm}$ ), on whole forebody or parts of it short setation apparent

- $\quad$ Smaller species (PL below $0.75 \mathrm{~mm}$, PW usually $<1.15 \mathrm{~mm}$ ), extremely short setae on forebody only apparent under high magnification 3

2 Head less transverse, temple with insignificant broadening immediately behind eye. Habitus (Fig. 71), aedeagus (Figs 19-21) [N India to Yunnan and $\mathrm{N}$ Thailand] A.apicipennis

- Head much more transverse and trapezoid, temple broadening posteriorly. Habitus (Fig. 88), aedeagus (Figs 157-159) [Indonesia: Sumatra]

A. jambi

3 Rim from the anterior margin of head continuing in ridge at the inner edge of eye and passing along it. Neck separated by transversal (usually arched) occipital furrow, tactile setae (variously developed) present on dosal side of head

- If slight rim at the anterior margin of head can be observed, it never continues at the inner edge of eye. Neck only separated from vertex by contrast of sculpture (usually rough punctation of head transforming into microsculpture along indistinct line)

4 Forebody with very dense scuplture, dull. Vertex with well-developed tactile setae (dark and erect) situated postero-medially of each eye 5

- Forebody with less conspicuously dense sculpture, with some lustre. Vertex with less developed, inconspicuous tactile setae situated postero-mediad of each eye

5 Much larger species ( $\mathrm{PW}=0.99-1.14 \mathrm{~mm}$ ), pronotal side margins and hind margin forming an unbroken arch, posterior corners inconspicuous. Habitus (Fig. 76), aedeagus (Figs 61-63) [India: Tamil Nadu]

A. coonoor 
- Small species $(\mathrm{PW}=0.65-0.70 \mathrm{~mm}$ ), pronotal hind margin more gently arched, posterior corners regularly rounded. Habitus (Fig. 75), aedeagus (Figs 129-131) [India: Meghalaya and S Thailand]

A. gunung

6 Usually smaller species with temples around 1.3× length of eyes $\quad 7$

- Generally larger or more elongate species, temples around $1.5 \times$ length of eyes

7 Sculpture on vertex composed of irregular spaces bordered by ridges like intertwined roots (areolate). Mostly dark coloured, dark brown with black head. Habitus (Fig. 74), aedeagus (Figs 33-35) [Malaysia: Sabah]

A. borneensis

- Sculpture on vertex composed of semi-angular cavities divided by thin partitions (alveolate). Mostly lighter coloured, medium brown, reddish. Habitus (Fig. 73), aedeagus (Figs 234-236) [Malaysia: Sabah]

A. schawalleri

8 Large and rather dark coloured, head always black (HW $>0.70 \mathrm{~mm}$ ), sculpture on head mostly longitudinal (parallel) lines overlaying punctation. Habitus (Fig. 72), aedeagus (Figs 115-117) [N India and Nepal to S China, ?Vietnam]

A. ganapati

- Somewhat smaller species (HW $<0.70 \mathrm{~mm}$ ), dark brown or reddish, sculpture on vertex lacking such directionality, mostly like intertwined roots (overlaying punctation)

9 Smaller species (HW $<0.62 \mathrm{~mm}$ ), anterior pronotal corner sharper, anterior $1 / 3$ of lateral margin in ca. $80^{\circ}$ angle with horizontal axis. Habitus (Fig. 77), aedeagus (Figs 171-173) [China: Yunnan and N Thailand]

A. linaxi

- Larger species (HW $>0.62 \mathrm{~mm}$ ), anterior pronotal corner more rounded, anterior $1 / 3$ of lateral margin in ca. $70^{\circ}$ angle with horizontal axis $\quad 10$

10 In males apex of sternite VIII (Fig. 44) deeper incised on both sides, middle lobe larger, triangular. Habitus (Fig. 78), aedeagus (Figs 47-49) [India: Meghalaya, China: Sichuan, Jiangxi and Jiangsu, Japan] A. chinensis

- In males apex of sternite VIII (Fig. 210) with smaller incisions on sides of a smaller middle lobe. Habitus (Fig. 79), aedeagus (Figs 213-215) [China: Sichuan and Jiangxi]

A. reitteri

11 Dorsal side of abdomen shiny (except last two visible tergites) and covered with strong, large punctures with indistinct borders strongly punctate 
- $\quad$ Dorsal side of abdomen microsculptured (to variable degrees) and more shallowly/finely punctured

12 Temples slightly broadening behind eye. Head broader (TW $=0.57-0.68$ $\mathrm{mm})$, pronotum broader $(\mathrm{PW}=0.79-0.88 \mathrm{~mm}$ ) and less convex in middle. Habitus (Fig. 80), aedeagus (Figs 40-42) [Sri Lanka] A. ceylonicus

- Temples straight on a short distance behind eye, or gradually narrowing, arched towards neck. Head narrower $(\mathrm{TW}=0.55-0.61 \mathrm{~mm})$, pronotum narrower $(\mathrm{PW}=0.74-0.81 \mathrm{~mm}$ ). Habitus (Fig. 81), aedeagus (Figs 192194) [Sri Lanka]

A. montanus

13 Small species (TW < 0.55 mm). Habitus (Fig. 94), aedeagus (Figs 206-208) [Himalaya to the Philippines and the Greater Sunda Islands; Sri Lanka; New Guinea] A. pseudopsinus

- $\quad$ Larger species (TW $>0.55 \mathrm{~mm}$ ) 14

14 Antennomere 1 smooth. Most species with short elytra (PL > SC) 15

- Antennomere 1 at least finely sculptured. Species regularly with normally developed elytra $(\mathrm{PL}<\mathrm{SC})$ but in rare cases brachypterous morphs with shorter elytra may exist

15 Pronotal hind margin of pronotum before posterior corners, at most, shallowly incised

- $\quad$ Pronotal hind margin of pronotum before posterior corners extremely incised (species endemic to Java)

16 Temples large, more or less evenly rounded 17

- Temples more narrowly rounded or have peculiar shape

17 Lighter coloured, yellowish. Habitus (Fig. 84), aedeagus (Figs 248-250) [China: Yunnan]

A. schuelkei

- Darker coloured, predominantly dark brown or even blackish

18 Pronotal side zigzagged. Habitus (Fig. 83), aedeagus (Figs 241-243) [Myanmar: Chin State]

A. schillhammeri

- $\quad$ Pronotal side evenly rounded (but alatiform). Habitus (Fig. 82), aedeagus (Figs 136-138) [C Nepal]

A. hartmanni

19 Temples with rather sharp angle. Habitus (Fig. 85), aedeagus (Figs 26-28) [E Nepal, India: Darjeeling, ?Sikkim]

A. bengalensis

- $\quad$ Temples not with sharp angle, more or less rounded 
20 Temples broadening behind eye and narrowly rounded. Habitus (Fig. 86), aedeagus (Figs 108-110) [C Nepal]

A. franzi

- Temples slightly broadening behind eye and more broadly rounded. Habitus (Fig. 87), aedeagus (Figs 150-152) [E Nepal] A. jaljulensis

21 Smaller species (HW $<0.70 \mathrm{~mm}$ ). Habitus (Fig. 89), aedeagus (Figs 143145) [Indonesia: Java]

- $\quad$ Larger species (HW $>0.70 \mathrm{~mm}$ )

22 Anterior pronotal corners blunt, elytral length (SC) significantly smaller than pronotal length (PL). Habitus (Fig. 90), aedeagus (Figs 178-180) [Indonesia: Java]

A. lucidulus

- Anterior pronotal corners rather sharp, elytral length (SC) roughly equal to pronotal length (PL). Habitus (Fig. 91), aedeagus (Figs 220-222) [Indonesia: Java]

A. riedeli

23 Anterior margin of clypeal area distinctly concave. Habitus (Fig. 92), aedeagus (Figs 5-7) [S India]

A. andrewesi

- Anterior margin of clypeal area truncate or convex

24 Smaller species ( $\mathrm{HW}<0.70 \mathrm{~mm}$ ) with relatively large eyes (temples $1.00-$ $1.25 \times$ eyes) and temples broadening (often sharply angled or bulging) behind

- $\quad$ Species either larger $(\mathrm{HW}>0.70 \mathrm{~mm}$ ) or smaller eyed or temples not broadening behind

25 Frontline of head slightly broken near clypeal borders and antennal sockets, temples often slightly bulging right behind eye, about the same length as eyes. Habitus (Fig. 102), aedeagus (Figs 122-124) [Thailand, Vietnam and Malaysia to Sumatra, Java and the Philippines] A.genalis

- Outline of head characteristic helmet-shaped, temples often sharply angled, slightly longer than eyes. Habitus (Fig. 103), aedeagus (Figs 12-14) [Malaysia incl. Borneo and Indonesia: Sumatra, Java, Sulawesi]

A. anguliceps

26 Temples not or very slightly broadening behind eyes 27

- Temples significantly broadening behind eyes 30

27 Temples insignificantly broadening behind eyes 28 
- Temples only slightly broadening behind eyes, head transverse and slightly trapezoid quadrangular with equally broadly rounded anterior and posterior corners (species endemic to Sulawesi)

28 Widest point of pronotum at anterior $1 / 3$ or before. Anterior pronotal corner truncate in ca. $45^{\circ}$ angle with horizontal axis. Habitus (Fig. 93), aedeagus (Figs 262-264) [S China and Vietnam] A. topali

- Widest point of pronotum behind middle of side or after. Anterior pronotal corner narrowly rounded. Habitus (Fig. 95), aedeagus (Figs 185187) [N India and Nepal to S China and Vietnam]

A. malaisei

29 Abdomen with shallow but distinct puncation, fine coriaceous microsculpture apparent from tergite VI on. Habitus (Fig. 96), aedeagus (Figs 227-229) [Indonesia: N Sulawesi]

A. rurukan

- $\quad$ Abdomen with such extremely dense coriaceous microsculpture that not even traces of punctation observable, opaque. Habitus (Fig. 97), aedeagus (Figs 255-257) [Indonesia: S Sulawesi] A. tanator

30 Punctures on vertex with significant shiny interspaces (foveate). Habitus (Fig. 98), aedeagus (Figs 164-166) [Indonesia: Sumatra, Java] A. kabasi

- $\quad$ Punctures on vertex with less interspace (lacunose)

31 Very dark and robust ( $\mathrm{TW}=0.80-0.90 \mathrm{~mm}$ ). Habitus (Fig. 99), aedeagus (Figs 199-201) [Malaysia: Borneo and ?Indonesia: Sulawesi] A. nigricans

- $\quad$ Reddish or dark brown, usually smaller $(\mathrm{TW}=0.63-0.84 \mathrm{~mm})$

32 Male with a pair of significant knobs or blunt spines on hind margin of sternite VII apex. Hind margin of elytra insignificantly incised before outer corner. Slightly smaller $(\mathrm{HW}=0.62-0.75)$, temples (sometimes angled, sometimes rounded) shorter ( $T L=0.18-0.21)$. Habitus (Fig. 100), aedeagus (Figs 54-56) [Pakistan to China incl. Taiwan, Peninsular Malaysia and the Philippines]

A. cimicoides

- Male with a pair of barely visible protuberances near hind margin of sternite VII apex. Hind margin of elytra conspicuously incised before outer corner. Slightly larger $(\mathrm{HW}=0.69-0.81)$, temples (sometimes angled, sometimes rounded) longer ( $\mathrm{TL}=0.20-0.24)$. Habitus (Fig. 101), aedeagus (Figs 68-70) [Nepal and NE India to Vietnam] A. excisicollis 


\section{Anotylus andrewesi (Cameron, 1930) \\ (Figs 1-7, 92)}

Oxytelopsis andrewesi Cameron, 1930: 207; Herman 1970: 413; Coiffait 1984: 151; Herman 2001: 1412.

Examined type material - Oxytelopsis andrewesi Cameron - Lectotype $(\widehat{0}$, here designated): "Lecto-; type [lilac margined disc, curator label] \ Type [red margined disc, cu-

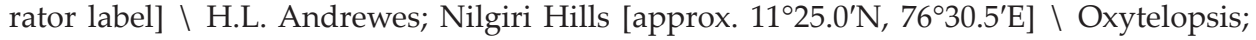
andrewesi; Type [underlined, in maroon] Cam. \ M. Cameron.; Bequest.; B.M. 1955-147. $\backslash$ Oxytelopsis; andrewesi Cam.; P.M. Hammond; det. 1970; Lectotype \ $\hat{0} \backslash$ Anotylus; andrewesi (Cameron); det. Makranczy, 2015" (BMNH); Paralectotypes (7): "Para-; lecto-; type [light blue margined disc, curator label] \ Nilgiris; S. India \M. Cameron.; Bequest.; B.M. 1955-147. \andrewesi [in pencil] \Oxytelopsis; andrewesi Cam.; P.M. Hammond; det. 1970; Paralectotype \Anotylus; andrewesi (Cameron); det. Makranczy, 2015" (1 ㅇ, $\mathrm{BMNH}$ ); "Para-; lecto-; type [light blue margined disc, curator label] \ Para-; type [yellow margined disc, curator label] \H.L. Andrewes; Nilgiri Hills \M. Cameron.; Bequest.; B.M. 1955-147. \Oxytelopsis; andrewesi Cam.; P.M. Hammond; det. 1970; Paralectotypes $\backslash$ Anotylus; andrewesi (Cameron); det. Makranczy, 2015” (2 O’, 4 क , BMNH).

Other material - INDIA: Kerala, Cardamom Hills, 5 km W Kumily, 1000 m, 6.XI.1972, leg. C. Besuchet, I. Löbl \& R. Mussard (12), tamisages en forêt (43, MHNG, 1, MNHP, 1, NIBR, 1, SMNS, 1, ZMHB, 1, CNCI, 1, ZMUC, 1, NMPC, 1, FMNH, 1, AMNH, 1, ISNB, 1, SDEI, 2, NHMW, 2, HNHM); Kerala, Cardamom Hills, entre Pambanar et Peermade, 950 m, 9.XI.1972, leg. C. Besuchet, I. Löbl \& R. Mussard (18), tamisages en forêt, près d'une rivière (2, MHNG); Kerala, Cardamom Hills, Muttapatti près de Munnar, 1700 m, 24.XI.1972, leg. C. Besuchet, I. Löbl \& R. Mussard (48), tamisages en forêt, au pied d'un groupe de fougères arborescentes (1, MHNG); Kerala, Nelliampathi Hills (NW Anaimalai Hills), Kaikatty, 900 m, 30.XI.1972, leg. C. Besuchet, I. Löbl \& R. Mussard (58), tamisages en forêt, dans un ravin (22, MHNG); Kerala, Kottiyoor, Wynaad Taluk, 650 m, 7.IV.1969, leg. Soans \& Brown (1, MHNG); Madras, Palni Hills, 7 km E Kodaikanal, 1750 m, 12.XI.1972, leg. C. Besuchet, I. Löbl \& R. Mussard (24), tamisages en forêt (11, MHNG); Madras, Anaimalai Hills, au-dessus d'Aliyar Dam, 550 m, 17.XI.1972, leg. C. Besuchet, I. Löbl \& R. Mussard (32), tamisages en forêt (2, MHNG); Madras, Nilgiri, 15 km E Coonoor, 900 m, 19.XI.1972, leg. C. Besuchet, I. Löbl \& R. Mussard (37), tamisages en forêt (1, MHNG); Madras, Nilgiri, 7 km E Coonoor, 1350 m, 19.XI.1972, leg. C. Besuchet, I. Löbl \& R. Mussard (38), tamisages en forêt (9, MHNG); Madras, Anaimalai Hills, Valparai, 1100 m [10¹9'03"N , 76 57'31"E], 20.XI.1972, leg. C. Besuchet, I. Löbl \& R. Mussard (39), tamisages dans une forêt avec caféiers (49, MHNG); Tamil Nadu, Anaimalai Hills, 700-1000 m, 17.I.1972, leg. R. Mussard (4) (4, MHNG); Madras, Amaravathi Dam, dans une vallée entre les Anaimalai Hills et les Palni Hills, 20 km S Udamalpet, 400 m, 26.XI.1972, leg. C. Besuchet, I. Löbl \& R. Mussard (53), au bord du lac (85, MHNG); Madras, Palni Hills, $16 \mathrm{~km}$ E de Kodaikanal [10¹7'28' N, 77³8’05”E], 1400 m, 15.XI.1972, leg. C. Besuchet, I. Löbl \& R. Mussard (28), tamisages en forêt (2, MHNG); Tamil Nadu, Kodaikanal, 1800 m, 8.I.1972, leg. R. Mussard (10) (2, MHNG); Tamil Nadu, Palni Hills, Perumalmalai, $10^{\circ} 16^{\prime}$ N, 77³3’E, 1600 m, 12.I.1999, leg. D. Boukal (61) (1, NHMW); Tamil Nadu, Nilgiri Hills, Thaishola, $1^{\circ} 14^{\prime} \mathrm{N}, 7^{\circ} 37^{\prime} \mathrm{E}, 2100 \mathrm{~m}$ 23.I.1999, leg. D. Boukal (81) (2, NHMW); Tamil Nadu, Shevaroy Hills, Yercaud, Kiliyur Falls env., $11^{\circ} 47^{\prime} \mathrm{N}, 78^{\circ} 12^{\prime} \mathrm{E}, 1400$ m, 25.I.1999, leg. D. Boukal (85) (1, NHMW, 1 ○̊, 1 ㅇ, HNHM). 


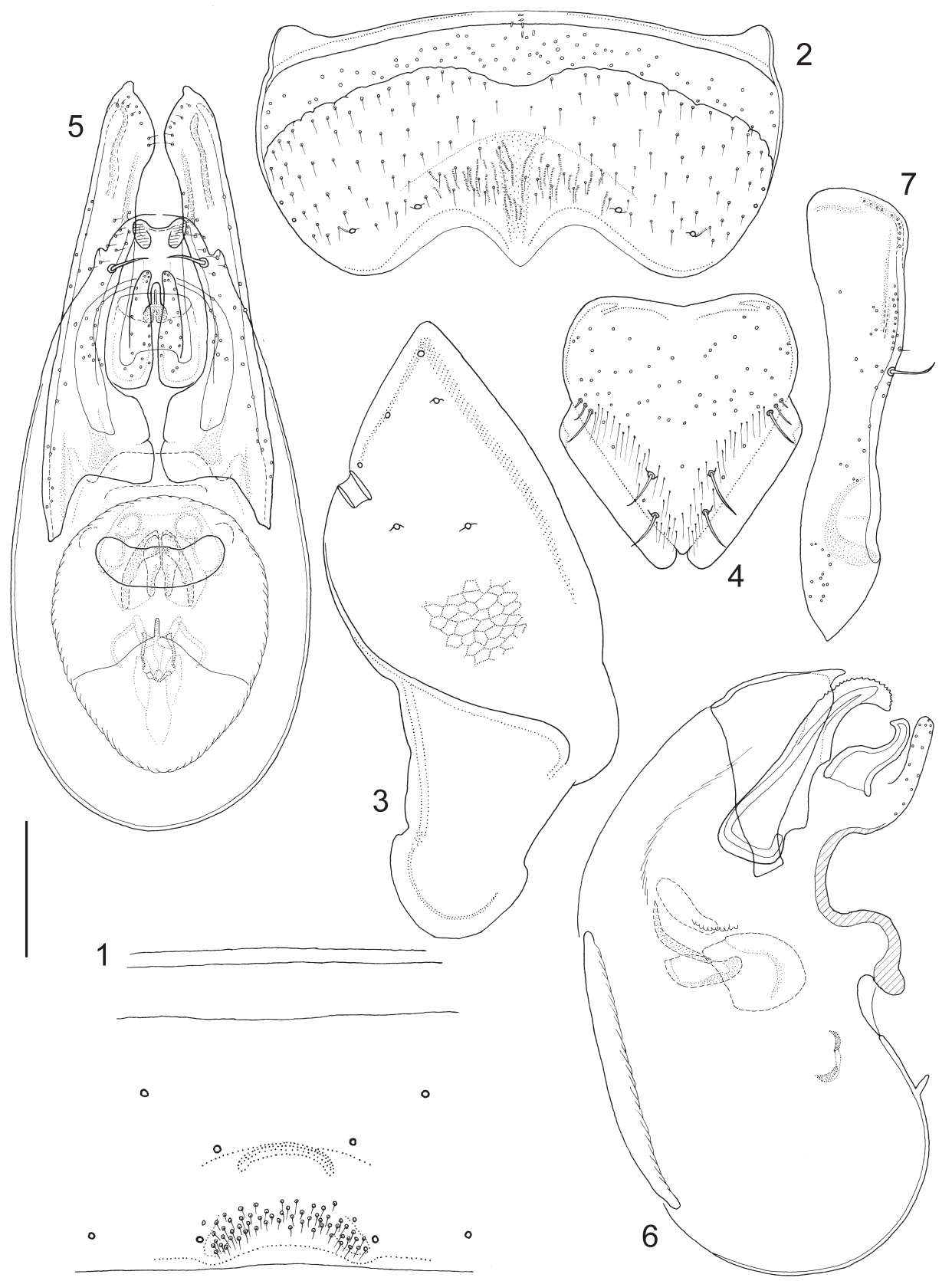

Figs 1-7. Anotylus andrewesi (Cameron, 1930), male. $1=$ median part of sternite VII, $2=$ sternite VIII, $3=$ tergite IX, $4=$ tergite $X, 5=$ aedeagus, frontal view, $6=$ median lobe, lateral view, 7 = paramere, lateral view. Scales: $0.10 \mathrm{~mm}$ for Figs 5-7, $0.12 \mathrm{~mm}$ for Figs 1, 3-4, 0.14 mm for Fig. 2 
Redescription - Measurements (in mm, n=10): HW $=0.61(0.58-0.64)$; TW $=0.58(0.55-$ $0.62) ; \mathrm{PW}=0.85(0.80-0.91) ; \mathrm{EW}=0.96(0.91-1.00) ; \mathrm{AW}=0.95(0.89-1.01) ; \mathrm{HL}=0.48(0.46-$ $0.51) ; \mathrm{EL}=0.14(0.13-0.15) ; \mathrm{TL}=0.18(0.17-0.19) ; \mathrm{PL}=0.48(0.45-0.50) ; \mathrm{SC}=0.58(0.54-0.62)$; $\mathrm{FB}=1.60$ (1.52-1.70); BL = 2.98 (2.78-3.30). Habitus as in Fig. 92. Forebody moderately shining despite sculpture, abdomen very finely microsculptured and with large but extremely shallow, obscured punctures, less lustrous. Elytra dark brown to black, head, pronotum, abdomen and legs dark brown, except explanate part of pronotum and transparent clypeal area lighter, mouthparts and antennomeres 2-4 medium brown, other antennomeres dark brown. Head with an extremely thin rim on anterior margin, latter rather distinctly concave in middle, slightly angled also at supraantennal prominences, these as moderately elevated oblique ridges, disc slightly impressed near eyes. Epistomal suture forming subcircular impression (similarly punctured inside), upper surface without tactile setae, eyes moderately small and somewhat bulging from sideline of head; in dorsal view temples (1.3× length of eye) alatiform, laterally rounded and posteriorly constricted to form distinct neck, however, dorsally separated only by rudimentary occipital furrow, more importantly by dorsum of neck being microsculptured rather than punctured. Antennomere 1 flattened club-shaped and lightly microsculptured, segment 2 elongate oval, segment 3 club-shaped, segment 4 almost round, segments 5-11 with basal dishes, segments 6-10 transverse (about $1.5 \times$ broader than long). Head and pronotum with mostly alveolate sculpture. Pronotum with lateral portion explanate and slightly up-turned, edge alatiform with sparse short setae; lateral margin unevenly arched with a number of small, irregularly distributed denticles, posterior margin rather strongly incised before corners; disc medially with two faint, posteriorly confluent longitudinal ridges, impressed alongside them, laterally impressed around middle of sides. Elytra with membranous posterior margin extending from sutural corner to 5/6 of hind margin. Elytral epipleural ridge present with up-turned lateral alatiform portions, disc longitudinally impressed alongside; shoulders well developed, surface foveolate, in between punctures torulose. Legs short, pro- and mesotibia with several spinulose rows, metatibia with longitudinal ctenidium of spinules in distal half. Abdomen with sides moderately arcuate, second segment with paratergites strongly broadening posteriorly (abdomen appearing constricted at base), those of segments III-VII thin, mesal paratergites broad; posterior edge of tergite VII with thin palisade fringe, median part of sternite VII as in Fig. 1, sternite VIII (Fig. 2), tergites IX (Fig. 3), $\mathrm{X}$ (Fig. 4) and aedeagus as in Figs 5-7.

Distribution - The currently known distribution is confined to more mountainous areas of southern India.

Remarks - The species is easily recognizable by the combination of the locality and the conspicuously concave frontal margin of the clypeal area.

\section{Anotylus anguliceps (Cameron, 1934)}

(Figs 8-14, 103)

Oxytelopsis anguliceps Cameron, 1934: 31; Cameron 1936: 30; Herman 1970: 413; Herman 2001: 1412.

Examined type material - Oxytelopsis anguliceps Cameron - Lectotype $(\hat{\sigma}$, here designated): “[on mounting card] $\hat{\partial} \backslash$ Lecto-; type [lilac margined disc, curator label] $\backslash$ Holo-; 


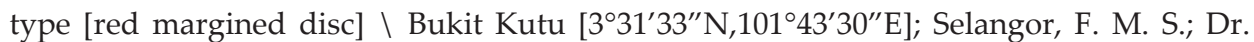
Cameron. \O.; anguliceps; Type [underlined, in maroon] Cam. \ M. Cameron.; Bequest.; B.M. 1955-147. \Oxytelopsis; anguliceps Cam.; P. M. Hammond; det. 1970; Lectotype \ Anotylus; anguliceps (Cameron); det. Makranczy, 2015" (BMNH); Paralectotypes (4): “[on mounting card] $\widehat{\partial} \backslash$ Para-; lecto-; type [light blue margined disc] \Bukit Kutu; Selangor, F. M. S.; Dr. Cameron \W. Steel coll.; B.M. 1969-552. \Oxytelopsis; anguliceps Cam.; P. M. Hammond; det. 1970; Paralectotype \Anotylus; anguliceps (Cameron); det. Makranczy, 2015” (1 ふ , BMNH); “[on mounting card] ô \ Para-; lecto-; type [light blue margined disc] $\backslash$ Bukit Kutu; Selangor, F. M. S.; Dr. Cameron \M. Cameron.; Bequest.; B.M. 1955-147 \ Oxytelopsis; anguliceps Cam.; P. M. Hammond; det. 1970; Paralectotype \Anotylus; anguliceps (Cameron); det. Makranczy, 2015” (1 $\widehat{\jmath}, \mathrm{BMNH})$; “[on mounting card] $q \backslash$ Para-; lecto-; type [light blue margined disc] \Rotting; Fruit \Bukit Kutu; Selangor, F. M. S.; Dr. Cameron \ M. Cameron.; Bequest.; B.M. 1955-147. \ Oxytelopsis; anguliceps Cam.; P. M. Hammond; det. 1970; Paralectotype \anguliceps [writing in red ink] \Anotylus; anguliceps (Cameron); det. Makranczy, 2015" (1 क, BMNH); Para-; lecto-; type [light blue margined disc] \Rotting; Fruit \Bukit Kutu; Selangor, F. M. S.; Dr. Cameron \M. Cameron.; Bequest.; B.M. 1955-147. \Oxytelopsis; anguliceps Cam.; P. M. Hammond; det. 1970; Paralectotype \Anotylus; anguliceps (Cameron); det. Makranczy, 2015" (1 §, BMNH).

Other material - MALAYSIA: Perak, Cameron Highlands, cascade Sungei Simei, 25.III.1977, leg. T. Jacoud (34i, 61i) (9, MHNG); Selangor F.M.S., Bukit Kutu, leg. M. Cameron (1, MHNG); W-, Lake Kenyir, 5 km SW dam, 50 km SW Kuala Terengganu 350 m, 7-12. VII.2001, leg. A Schulz \& K. Vock (1, SMNS); MALAYSIA/BORNEO: Sabah, Sepilok Forest Res. (near Sandakan), 8.VI.1968, leg. R.W. Taylor, ex humus (1, MHNG); Sabah, Mt. Kinabalu National Park, above Poring Hot Springs, 520 m, 15.VIII.1988, leg. A. Smetana (B115), sifting of layers of fermenting fruits (from a tree, Ficus sp.) on forest floor and debris under (12, FMNH, 1, ISNB, 1, SDEI, 1, NHMW, 1, MNHP, 1, MHNG, 1, HNHM); Sabah, Mt. Kinabalu National Park, Poring Hot Springs, 495 m, 21.VIII.1988, leg. A. Smetana (B136), sifting of layers of fermenting fruits (from a tree, Ficus sp.) on forest floor and debris under (2, FMNH), same but $485 \mathrm{~m}$ (B135) sifting of accumulated old fruits of Areca palms, including debris and humus underneath, open area (2, FMNH, 1, HNHM); Sabah, Mt. Kinabalu National Park, above Poring Hot Springs, 520 m, 22.VIII.1988, leg. A. Smetana (B139), sifting of layers of rotting fruits (Ficus sp.) on forest floor and debris under (2, FMNH); Sabah, Mt. Kinabalu National Park, Poring Hot Springs, 495 m, 23.VIII.1988, leg. A. Smetana (B140), sifting of layers of fallen fruits in primary tropical rain forest (20, FMNH, 1, NIBR, 1, IZAS, 1, ZMHB, 1 ऽ, 1, HNHM), same but $475 \mathrm{~m}$ (B141) sifting of old fruits of Ficus tree, accumulated on and around a humongous rotten tree on ground in rain forest (1, FMNH); Sabah, Mt. Kinabalu National Park, Poring Hot Springs, 495 m, 24.VIII.1988, leg. A. Smetana (B144), sifting of layers of fallen fruits in primary tropical rain forest (4, FMNH, 1, CNCI, 1, AMNH), same but 30.VIII.1988 (B161), sifting of three large fermenting fruits (cocoa nut size) and debris under them in rain forest (1, FMNH), same but $485 \mathrm{~m}, 25$. VIII.1988 (B147), sifting of accumulated old fruits of Areca palms, including debris and humus underneath, open area (1, FMNH); Sabah, Poring Hot Springs, 500 m env., forêt de Dipterocarpaceae, 7.V.1987, leg. D. Burckhardt \& I. Löbl (15a), tamisage dans la forêt (1, MHNG); INDONESIA/SUMATRA: West Sumatra, Bukittingi, Panti, 700 m, 25-26.X.1991, leg. A. Riedel, (1 ô, 2 , , SMNS, 1 ふ , ZMUC); INDONESIA/JAVA: 50 km S Surabaya, Tretes, Kekek Bodo WF, $800 \mathrm{~m}, 20 . I X .1995$, leg. H. Schillhammer (1a), sifted from very wet leaf litter at the base of a rock wall (4, NHMW); INDONESIA/SULAWESI: N-, Gunung Ambang NSG, 20.IV.1992, leg. S. Schödl (13) (2, NHMW); C-, Palu, Palolo, Kamarora trail to waterfall, 700 m, 23-27. VIII.1997, leg. A. Riedel (2, NHMW). 


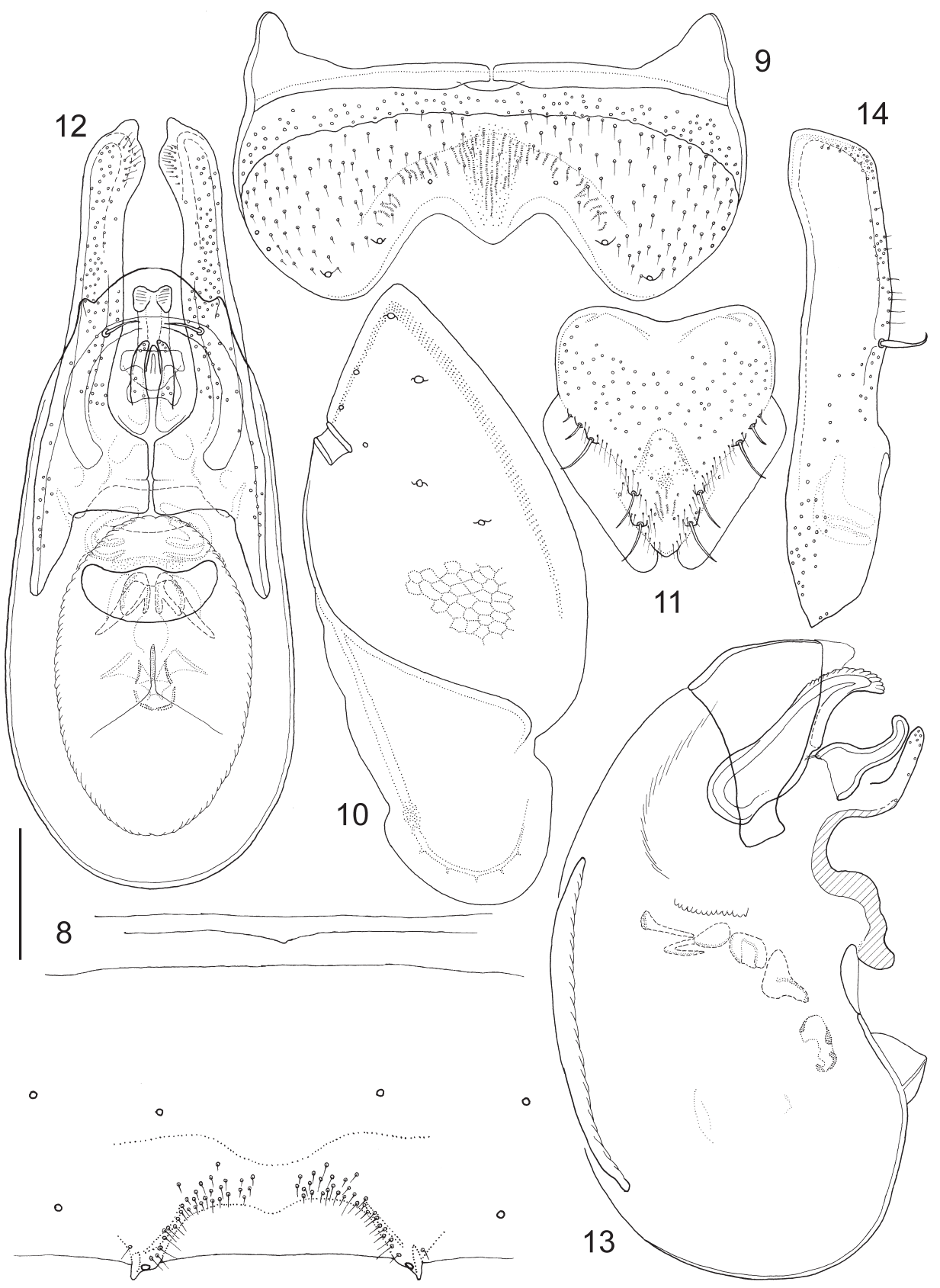

Figs 8-14. Anotylus anguliceps (Cameron, 1934), male. $8=$ median part of sternite VII, $9=$ sternite VIII, $10=$ tergite IX, 11 = tergite $X, 12$ = aedeagus, frontal view, $13=$ median lobe, lateral view, 14 = paramere, lateral view. Scales: $0.10 \mathrm{~mm}$ for Figs 12-14, $0.12 \mathrm{~mm}$ for Figs 8, 11, $0.135 \mathrm{~mm}$ for Fig. 10, $0.16 \mathrm{~mm}$ for Fig. 9 
Redescription - Measurements (in mm, $\mathrm{n}=10)$ : $\mathrm{HW}=0.63(0.60-0.68) ; \mathrm{TW}=0.64(0.60-$ $0.70) ; \mathrm{PW}=0.89$ (0.85-0.98); $\mathrm{EW}=1.00(0.95-1.11) ; \mathrm{AW}=0.99(0.91-1.15) ; \mathrm{HL}=0.48(0.46-$ $0.52) ; \mathrm{EL}=0.15(0.14-0.16) ; \mathrm{TL}=0.18(0.17-0.19) ; \mathrm{PL}=0.51(0.48-0.54) ; \mathrm{SC}=0.59(0.55-0.68)$; $\mathrm{FB}=1.66(1.58-1.82) ; \mathrm{BL}=3.23(2.96-3.42)$. Habitus as in Fig. 103. Forebody moderately shining despite sculpture, abdomen very finely microsculptured, densely but finely and shallowly punctured, less lustrous. Body reddish dark brown, except mouthparts and antennomeres 1-4 reddish medium brown. Head with anterior margin forming an unbroken arch with slight rim, supraantennal prominences as moderately elevated oblique ridges, disc slightly impressed near eyes, middle of vertex slightly elevated. Epistomal suture forming subcircular impression (less sculptured inside), upper surface without tactile setae, eyes rather large, moderately convex; in dorsal view temples (1.2× length of eye) alatiform, laterally bluntly or sharply angled and posteriorly constricted to form distinct neck, however, dorsal side not separated by occipital furrow, rather by dorsum of neck bearing microsculpture instead of punctation. Antennomere 1 flattened club-shaped and finely microsculptured, segment 2 broad at base and narrowing apically segment 3 stout club-shaped, segment 4 slightly transverse, segments 5-11 with basal dishes, segments 6-10 transverse (almost $1.5 \times$ broader than long). Head and pronotum with mostly alveolate sculpture. Pronotum with lateral portion explanate and slightly up-turned, edge alatiform with sparse short setae; lateral border uneven and with a number of small, irregularly distributed denticles, posterior margin slightly incised before corners; disc medially with two parallel and posteriorly almost confluent longitudinal ridges, posteriorly impressed alongside them, laterally strongly impressed around middle of sides. Elytra with membranous posterior margin extending from sutural corner to 5/6 of hind margin. Elytral epipleural ridge present with up-turned lateral alatiform portions, disc longitudinally impressed alongside; shoulders well developed, surface foveolate, in between punctures torulose. Legs short, pro- and mesotibia with several spinulose rows, metatibia with longitudinal ctenidium of spinules in distal half. Abdomen with sides gently arcuate, second segment with paratergites strongly broadening posteriorly (abdomen appearing constricted at base), those of segments III-VII thin, mesal paratergites broad; posterior edge of tergite VII with thin palisade fringe, median part of sternite VII as in Fig. 8, sternite VIII (Fig. 9), tergites IX (Fig. 10), X (Fig. 11) and aedeagus as in Figs 12-14.

Distribution - Currently known from Malaysia and Indonesia (Sumatra, Java, Sulawesi), appears to be rather widespread.

Remarks - The species seems to prefer rotting/fermenting fruits and soaking wet leaf litter.

\section{Anotylus apicipennis (Fauvel, 1895) (Figs 15-21, 71)}

Oxytelopsis apicipennis Fauvel, 1895: 200; Cameron 1930: 204; Herman 1970: 413; Coiffait 1984: 150; Herman 2001: 1413.

Examined type material - Oxytelopsis apicipennis Fauvel - Lectotype ( + , here designated): "Lecto-; type [lilac margined disc, curator label] \[Mt.] Carin; Asciuii Ghecù [19¹7.0’N, 96²4.5'E]; 1400-1500 m.; L. Fea. III-IV.[18]88. \ apicipennis; Fvl. \ Coll. et det. A. Fauvel; Oxytelopsis; apicipennis Fauv.; R.I.Sc.N.B. $17.479 \backslash$ Syntype $\backslash$ Oxytelopsis; api- 
cipennis Fvl.; P.M. Hammond; det. 1972; Lectotype $\backslash q \backslash$ Anotylus; apicipennis (Fauvel); det. Makranczy, 2015" (ISNB).

Other material - NEPAL: Dhaulagiri Himal, S-slope, N Banduk vill., 1900-2300 m, $28^{\circ} 27^{\prime} 22^{\prime \prime} \mathrm{N} 83^{\circ} 35^{\prime} 13^{\prime \prime} \mathrm{E}$ to $28^{\circ} 28^{\prime} 07^{\prime \prime} \mathrm{N} 83^{\circ} 35^{\prime} 10^{\prime \prime} \mathrm{E}, 06 . V .2009$, leg. J. Schmidt (7, NKME); Manaslu Mts., S of Bara Pokhari, 2300 m, 08.IV.2003, leg. J. Schmidt (1, NKME); Manaslu Mts., S Bara Pokhari, ca. $28^{\circ} 15^{\prime} \mathrm{N}, 8^{\circ} 25^{\prime}$ E, 2100 m, 29.IV.2005, leg. J. Schmidt (3, NKME); Manaslu Mts., Bara Pokhari Lekh above Bhachok Goan village, $28^{\circ} 14^{\prime} 28^{\prime \prime} \mathrm{N}, 84^{\circ} 24^{\prime} 32^{\prime \prime} \mathrm{E}$, 1600-1800 m, 29.IV.2005, leg. J. Schmidt (3, NKME); N Kathmandu, Shivapuri Lekh, upper Bagmati river valley, 1700-1800 m, 24.V.2005, leg. J. Schmidt (2, NKME); INDIA: West Bengal, Darjeeling distr., Algarah, 1800 m, 9.X.1978, leg. C. Besuchet \& I. Löbl (6), tamisage en forêt (1, MHNG); West Bengal, Darjeeling distr., entre Algarah et Labha, à 7 km d'Algarah, versant sud, 1900 m, 11.X.1978, leg. C. Besuchet \& I. Löbl (10), tamisage en forêt (5, MHNG, 1, HNHM); Meghalaya, Khasi Hills, au-dessus de Shillong, N-slope, 1850-1950 m, forêt primaire du Shillong Peak, 25.X.1978, leg. C. Besuchet \& I. Löbl (27), tamisage en forêt (4, MHNG); Meghalaya, Khasi Hills, Mawsynram-Balat, 1000 m, 27.X.1978, leg. C. Besuchet \& I. Löbl (30b), tamisage en forêt (1, MHNG); Meghalaya, Khasi Hills, Weiloi $\left[25^{\circ} 21^{\prime} \mathrm{N}\right.$, 913'ㄹ E], 1700 m, 27.X.1978, leg. C. Besuchet \& I. Löbl (31), tamisage en forêt (8, MHNG, 2, HNHM); Meghalaya, Khasi Hills, au-dessus de Shillong, 1850-1950 m, forêt primaire du Shillong Peak, 30.X.1978, leg. C. Besuchet \& I. Löbl (35b), tamisage en forêt (5, MHNG); Meghalaya, Garo Hills, au-dessus de Tura, 700-900 m, forêt du Tura Peak, 1.XI.1978, leg. C. Besuchet \& I. Löbl (37b), tamisage en forêt (3, MHNG); NE-, Arunachal Pr. (South Tibet), Etalin vicinity, $28^{\circ} 36^{\prime} 56^{\prime \prime} \mathrm{N}, 9^{\circ} 53^{\prime} 21^{\prime \prime} \mathrm{E}, 700 \mathrm{~m}, 15-25 . V .2012$, leg. L. Dembický, sifting + Winkler app. extraction (2, ZFMK); Assam, North Cachar Hills distr., Mt. Borail, Borail Peak, $25^{\circ} 06^{\prime} 43^{\prime \prime} \mathrm{N}, 93^{\circ} 03^{\prime} 11^{\prime \prime} \mathrm{E}, 1700 \mathrm{~m}, 19 . \mathrm{X} .2005$, leg. G. Cuccodoro \& A. Marletta (8a), sifting bamboo leaf litter in mountain rainforest (12, MHNG); MYANMAR: Shan State, ca. 35 km N Aungban, Mintaingbin Forest Camp, primary riverine forest, extending ca. $50-100 \mathrm{~m}$ from Mintaingbin river, peripheric area cultivated, $20^{\circ} 55.20^{\prime} \mathrm{N}, 96^{\circ} 33.60^{\prime} \mathrm{E}$, ca. $1320 \mathrm{~m}, 11-$ 23.VI.2004, leg. H. Schillhammer, H. Shaverdo, \& U Myint Hlaing (146a), FIT (1, NHMW); Mandalay Region, Mogok Township, S Panlin vill., Mt. Taung Mae, west slope, 1710-1750 $\mathrm{m}$, ca. $22^{\circ} 58^{\prime} 09^{\prime \prime} \mathrm{N}, 9^{\circ} 27^{\prime} 13^{\prime \prime} \mathrm{E}, 10-18$.VI.2014, leg. A. Brunke \& H. Schillhammer (MBS 201a), sifted from wet leaf litter (11, NHMW); Mandalay Region, ca. 7 km NW Mogok, above road Mogok-Bernardmyo, S Panlin village, west slope of Mt. Taung Mae, ca. $22^{\circ} 58^{\prime} 05.8^{\prime \prime} \mathrm{N}, 9^{\circ} 27^{\prime} 29^{\prime \prime} \mathrm{E}$, ca. 1870 m, 17-24.V.2016, leg. H. Schillhammer, A. Brunke, J. Jenkins Shaw, A. Jensen (212b), slightly disturbed primary forest, sifting of leaf litter $(4 \hat{\delta}, 1 \propto$, 16, NHMW, 2 ठ, HNHM); CHINA: Yunnan, E Kunming, Xiaobailong Forest Park, $24^{\circ} 55^{\prime} 43^{\prime \prime} \mathrm{N}, 103^{\circ} 05^{\prime} 22^{\prime \prime} \mathrm{E}, 2110 \mathrm{~m}, 10 . \mathrm{VIII} .2014$, leg. V. Assing (3) secondary pine forest, pine litter and litter at trail margin sifted (1, coll. Assing); Yunnan, mountains S Jianshui, $23^{\circ} 25^{\prime} 20^{\prime \prime} \mathrm{N}, 102^{\circ} 51^{\prime} 05^{\prime \prime} \mathrm{E}, 1890 \mathrm{~m}$, 22.VIII.2014, leg. V. Assing (18), subtropical broad-leaved forest, litter sifted (6, coll. Assing); Yunnan, W-, 60 km E Tongchong, 2300 m, 14-19.V.2006, [leg.] S. Murzin \& I. Shokhin (1, coll. Schülke); Yunnan, Baoshan Pref., Gaoligong Shan, nr. Xiaoheishan N. R., 35 km SE Tengchong, 2450'16"N, 9845'43”'E, 2110 m, 30.V/4.VI.2007, leg. D.W. Wrase (11), primary decid. forest, litter sifted (1, coll. Schülke); Yunnan, Baoshan Pref., Gaoligong Shan, nr. Xiaoheishan N. R., 35 km SE Tengchong, 2450'16" N, 98 $45^{\prime} 43^{\prime \prime}$, 2110 m, 30.V.2007, leg. M. Schülke (CH07-11), deciduous forest, litter sifted (1, coll. Schülke); Yunnan, Baoshan Pref., Gaoligong Shan, nr. Xiaoheishan N. R., 35 km SE Tengchong, $24^{\circ} 50^{\prime} 16^{\prime \prime} \mathrm{N}, 9^{\circ} 45^{\prime} 43^{\prime \prime} \mathrm{E}, 2110 \mathrm{~m}, 4 . V I .2007$, leg. M. Schülke (CH07-11B), deciduous forest, litter sifted (1, coll. Schülke); Yunnan, Baoshan Pref., mountain range $14 \mathrm{~km}$ E Tengchong, $25^{\circ} 00^{\prime} 28^{\prime \prime} \mathrm{N}, 98^{\circ} 38^{\prime} 07^{\prime \prime} \mathrm{E}, 1850 \mathrm{~m}, 1 . \mathrm{VI} .2007$, leg. M. Schülke (CH07-16), secondary mixed 
forest, litter sifted (1, coll. Schülke); Yunnan, Baoshan Pref., mountain range $25 \mathrm{~km} \mathrm{~S} \mathrm{Teng-}$ chong, 2448'28"N, 98³2'03"E, 1900 m, 2.VI.2007, leg. M. Schülke (CH07-17), devastated primary deciduous forest, litter, fungi sifted (2, coll. Schülke); Yunnan, Baoshan Pref., mountain range $22 \mathrm{~km} \mathrm{~S}$ Tengchong, $24^{\circ} 49^{\prime} 29^{\prime \prime} \mathrm{N}, 9^{\circ} 29^{\prime} 27^{\prime \prime} \mathrm{E}, 1750 \mathrm{~m}, 2$.VI.2007, leg. M. Schülke (CH07-18), secondary forest, litter, dead wood, sifted (2, coll. Schülke); Yunnan,

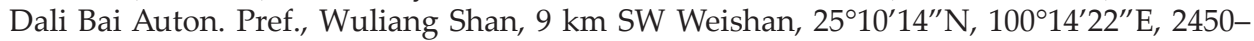
2500 m, 13.VI.2007, leg. M. Schülke (CH07-35), W slope, oaks and pines, sifted (2, coll. Schülke); Yunnan, Dali Bai Auton. Pref., Wuliang Shan, $9 \mathrm{~km} \mathrm{SW}$ Weishan, $25^{\circ} 10^{\prime} 14^{\prime \prime} \mathrm{N}$, 100¹4'22"E, 2450-2500 m, 13.VI.2007, leg. D.W. Wrase (35), W slope secondary oak/pine forest, pasture, under stones (1, coll. Schülke); Yunnan, Baoshan Pref., Gaoligong Shan, W

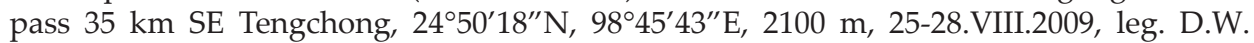
Wrase (06), devastated primary decid. forest, litter sifted (1, coll. Schülke); Yunnan, Baoshan Pref., Gaoligong Shan, W pass 35 km SE Tengchong, $24^{\circ} 50^{\prime} 18^{\prime \prime} \mathrm{N}, 98^{\circ} 45^{\prime} 43^{\prime \prime} \mathrm{E}, 2100$ m, 25.VIII.2009, leg. M. Schülke (CH09-06), devastated primary deciduous forest, litter, wood, mushrooms, sifted (1, coll. Schülke); Yunnan, Baoshan Pref., Gaoligong Shan, 65 km

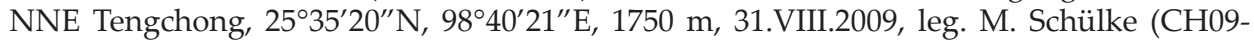
$10 \mathrm{~b})$, secondary mixed forest, stone debris, litter and moss, sifted (10, coll. Schülke); Yunnan, Baoshan Pref., mountain range 25 km S Tengchong, 24 $48^{\prime} 21^{\prime \prime} \mathrm{N}, 98^{\circ} 32^{\prime} 05^{\prime \prime} \mathrm{E}, 1900 \mathrm{~m}$, 30.VIII.2009, leg. M. Schülke (CH09-18), cleft with devastated primary forest, litter \& mushroom sifted (1, coll. Schülke); Yunnan, Baoshan Pref., Gao-ligong Shan, 78km N Teng-

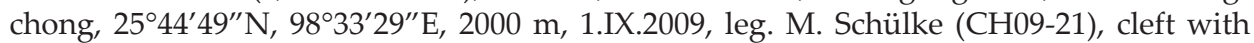
creek and forest remnant, litter \& dead wood sifted (2, coll. Schülke, 1, NMPC); Yunnan, Lincang Pref., Bangma Shan, 33 km SSW Lincang, 233' $41^{\prime \prime} \mathrm{N}, 100^{\circ} 00^{\prime} 27^{\prime \prime} \mathrm{E}, 2150 \mathrm{~m}, 11$. IX.2009, leg. D.W. Wrase (42), deciduous forest remnant, N slope, litter/moss sifted (1, coll. Schülke); Yunnan, Lincang Pref., Bangma Shan, 33km SSW Lincang, $23^{\circ} 35^{\prime} 41^{\prime \prime} \mathrm{N}$, $100^{\circ} 00^{\prime} 27^{\prime \prime} \mathrm{E}, 2150 \mathrm{~m}$, 11.IX.2009, leg. M. Schülke (CH09-42), deciduous forest remnant, N slope, litter and dead wood, sifted (3, coll. Schülke); Yunnan, Lincang Pref., Xue Shan, 48 km N Lincang, $24^{\circ} 19^{\prime} 03^{\prime \prime} \mathrm{N}, 100^{\circ} 07^{\prime} 13^{\prime \prime} \mathrm{E}, 2070 \mathrm{~m}$, 12.IX.2009, leg. M. Schülke (CH09-45), forest remnant, N slope, litter \& mushrooms sifted (17, coll. Schülke); THAILAND: Chiang Mai prov., Doi Suthep, versant sud, 1450 m, 4.XI.1985, leg. D. Burckhardt \& I. Löbl (5), tamisage de débris végétaux dans un ravin assez humide (15, MHNG); Chiang Mai prov., Doi Suthep, versant nord, 1550 m, 4.XI.1985, leg. D. Burckhardt \& I. Löbl (8), tamisage de feuilles mortes dans un petit ravin avec ruisselet (2, MHNG); Chiang Mai, Doi Suthep, versant nord, 1400 m, 5.XI.1985, leg. D. Burckhardt \& I. Löbl (11), ravin très humide, tamisage de feuilles mortes près d'un ruisseau (3, MHNG); Chiang Mai, Doi Inthanon, ravin en forêt, 1720 m, 7.XI.1985, leg. D. Burckhardt \& I. Löbl (15), tamisage de débris végétaux (3, MHNG); Chiang Mai, Doi Inthanon, ravin très humide, 1650 m, 7.XI.1985, leg. D. Burckhardt \& I. Löbl (16), tamisage de débris végétaux et de champignons près d'un ruisselet (54, MHNG); prov. Chiang Mai, Doi Inthanon, 1780 m, 17.XII.1986, leg. P. Schwendinger (1, MHNG); N-, CW Chiang Mai, Doi Inthanon, 1800 m, 14.V.2006, leg. R. Grimm (4, SMNS); Doi Inthanon, Maeaum, $18^{\circ} 32^{\prime} \mathrm{N}, 98^{\circ} 31^{\prime} \mathrm{E}, 1560 \mathrm{~m}, 18 . X \mathrm{XI} .2013$, leg. Ob (1), sifted near stream (1, coll. Assing); Doi Inthanon, Mae Aum, 18³1’ N, 98 $30^{\prime} \mathrm{E}, 1640$ m, 11.I.2014, leg. Ob (26), moist evergreen forest (1, coll. Assing); Doi Inthanon, Gate 2, 18 $8^{\circ} 32^{\prime} \mathrm{N}, 98^{\circ} 30^{\prime} \mathrm{E}, 1570 \mathrm{~m}$, 14.I.2014, leg. Ob (28), moist primary forest (1, coll. Assing); Doi Pha Hom Pok, Kiew Lom, $20^{\circ} 03^{\prime} \mathrm{N}, 9^{\circ} 09^{\prime} \mathrm{E}, 1935 \mathrm{~m}, 22 . \mathrm{I} .2014$, leg. Ob (55), leaf litter sifted (1, coll. Assing); Mae Hong Son prov., Ban Maeo Microwave, 1250 m, 15.XII.1990, leg. P. Schwendinger (1, MHNG). 


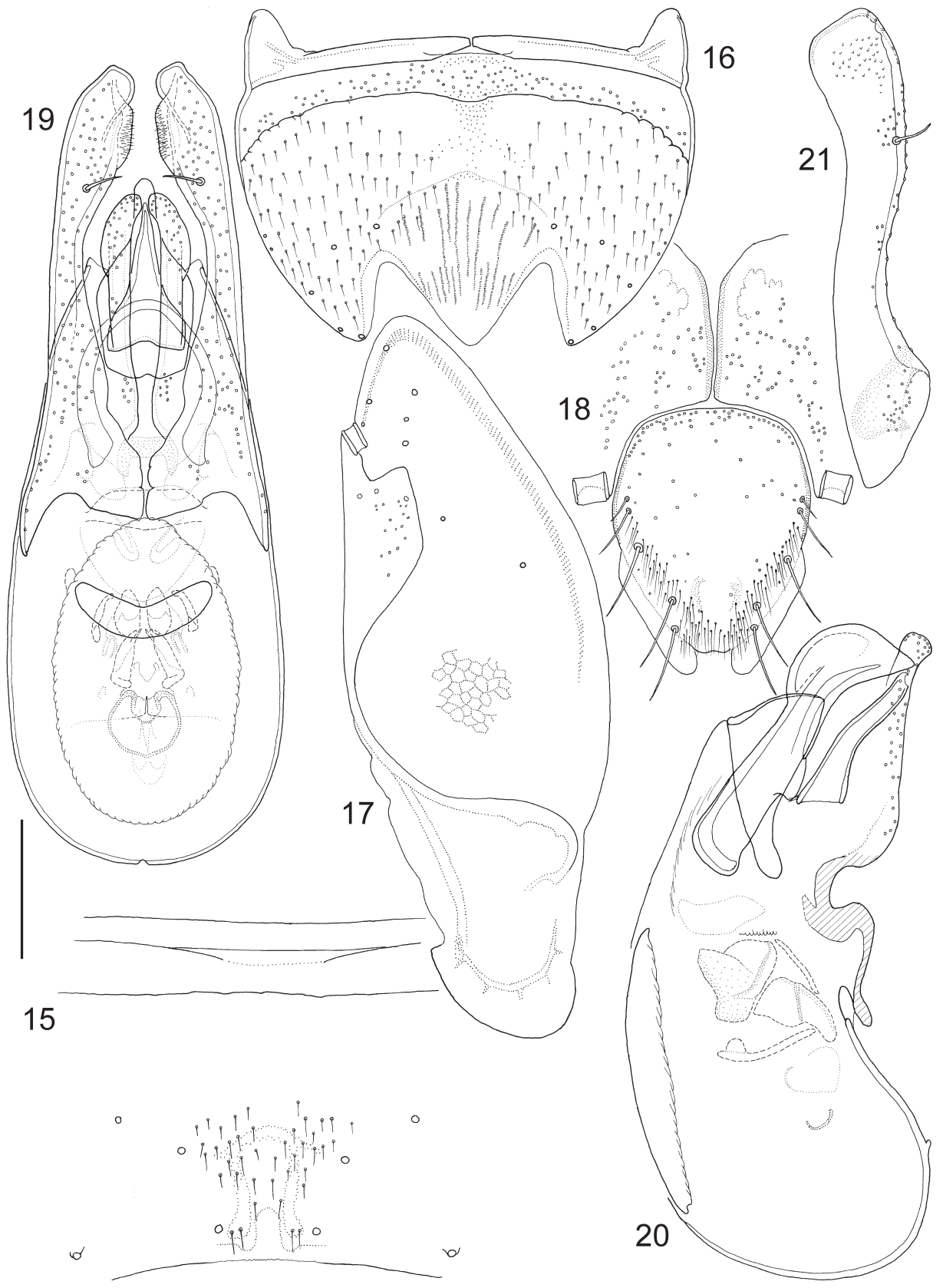

Figs 15-21. Anotylus apicipennis (Fauvel, 1895), male. 15 = median part of sternite VII, $16=$ sternite VIII, 17 = tergite IX, 18 = tergite $X, 19=$ aedeagus, frontal view, $20=$ median lobe, lateral view, 21 = paramere, lateral view. Scales: $0.175 \mathrm{~mm}$ for Fig. 18, $0.20 \mathrm{~mm}$ for Figs 15, 19-21, $0.22 \mathrm{~mm}$ for Fig. 17, $0.29 \mathrm{~mm}$ for Fig. 16 
Redescription - Measurements (in mm, $\mathrm{n}=10)$ : $\mathrm{HW}=0.93(0.86-0.96)$; $\mathrm{TW}=0.87(0.81-$ $0.92) ; \mathrm{PW}=1.22$ (1.14-1.27); $\mathrm{EW}=1.36$ (1.28-1.43); $\mathrm{AW}=1.37$ (1.28-1.43); $\mathrm{HL}=0.71$ (0.68$0.73) ; \mathrm{EL}=0.185(0.17-0.20) ; \mathrm{TL}=0.26(0.24-0.27) ; \mathrm{PL}=0.82(0.78-0.86) ; \mathrm{SC}=0.93(0.87-0.99)$; $\mathrm{FB}=2.58(2.48-2.69) ; \mathrm{BL}=5.30(4.92-5.46)$. Habitus as in Fig. 71. Forebody moderately shining despite sculpture, abdomen very finely microsculptured, less lustrous. Body reddish dark brown (or even blackish), except mouthparts reddish medium brown. Head with thin rim on anterior margin, latter truncate in middle, very slightly angled also at supraantennal prominences, these as elevated oblique ridges, disc impressed on small area near eyes. Epistomal suture forming subcircular impression (almost unpunctured inside), upper surface with weakly developed tactile setae (intratemporalis, situated postero-medially of each eye), eyes small (unusually situated posterior to middle of head length), slightly bulging from sideline of head; in dorsal view temples (1.4× length of eye) alatiform, laterally rounded and posteriorly constricted to form distinct neck, demarcated by occipital furrow, the ridge continues from inner side of eye to lateral part of anterior neck, then along (anteriad) the occipital furrow; the part of temple laterad ridge conspicuously setose. Antennomere 1 club-shaped, finely sculptured, segment 2 oval, segment 3 club-shaped, segment 4 small and isodiametrical, segments 5-11 with basal dishes, articles 6-10 isodiametrical (about as long as broad), segment 11 somewhat lighter at apex. Head and pronotum with mostly lacunose sculpture. Pronotum with lateral portion explanate and slightly up-turned, edge alatiform with very sparse short setae; lateral border evenly arched with marginal ridge and a number of small, regularly distributed denticles, posterior margin before corners concave but not incised; disc medially with two almost parallel longitudinal ridges nearly confluent posteriorly, impressed alongside them, laterally impressed near side margin. Elytra with membranous posterior margin extending from sutural corner to 5/6 of hind margin. Elytral epipleural ridge apparent as stronger sculpture lines in lateral alatiform portions, disc somewhat impressed in centre; shoulders well developed, surface foveolate, in between punctures torulose. Legs short, pro- and mesotibia with several spinulose rows, metatibia with longitudinal ctenidium of spinules in distal half. Abdomen with sides moderately arcuate, second segment with paratergites broadening posteriorly (abdomen appearing constricted at base), those of segments III-VII thin, mesal paratergites broad, posterior edge of tergite VII with thin palisade fringe, median part of sternite VII as in Fig. 15, sternite VIII (Fig. 16), tergites IX (Fig. 17), X (Fig. 18) and aedeagus as in Figs 19-21.

\section{Distribution - The distribution ranges from the Himalaya to northern Thailand.}

Remarks - The largest species of the species group, also notable as one with different shape of aedeagus and structure of terminalia from the rest of the species.

\section{Anotylus bengalensis (Biswas et Sengupta, 1980)} (Figs 22-28, 85)

Oxytelopsis bengalensis Biswas et Sengupta, 1980: 394; Herman 2001: 1413.

Examined material - INDIA: West Bengal, Darjeeling distr., Tigerhill, 2200-2300 m, 13.X.1978, leg. C. Besuchet \& I. Löbl (13), S-slope, sifted forest floor litter (57, MHNG, 2 §., HNHM, 1, FMNH); West Bengal, Darjeeling distr., Tonglu sommet et près du sommet, 
3100 m, 16.X.1978, leg. C. Besuchet \& I. Löbl (16b), tamisage dans une petite forêt et au pied d'arbustes dans le pâturage (1, MHNG); West Bengal, Darjeeling distr., Tonglu, Nslope, 2700 m, 16.X.1978, leg. C. Besuchet \& I. Löbl (17), tamisage en forêt (21, MHNG, 1, ZMUC, 1, SDEI, 1, NMPC, 1, AMNH); West Bengal, Darjeeling distr., Tigerhill, 2500-2600 m, 18.X.1978, leg. C. Besuchet \& I. Löbl (19), near summit, sifted forest floor litter (190, MHNG, 1, NIBR, 1, CNCI, 1, ISNB, 1, MNHP, 1, BMNH, 1, NHMW, 1 o, SMNS, 1 के, ZMHB, 1 ô, NKME); NEPAL: Ilam distr., nördl. Mai Pokhari, Tal der Gitang Khola, 2550 m, 28-31.III.1980, leg. J. Martens \& A. Ausobsky (121a), ursprünglicher Lithocarpus pachyphyllaWald, Berlese (3, SMNS, 1 q, HNHM).

Redescription - Measurements (in mm, $\mathrm{n}=10)$ : $\mathrm{HW}=0.63(0.60-0.66)$; $\mathrm{TW}=0.64(0.61-$ $0.67) ; \mathrm{PW}=0.84(0.80-0.90) ; \mathrm{EW}=0.84(0.79-0.89) ; \mathrm{AW}=0.90(0.85-0.95) ; \mathrm{HL}=0.47(0.44-$ $0.51) ; \mathrm{EL}=0.10(0.09-0.105) ; \mathrm{TL}=0.18(0.16-0.19) ; \mathrm{PL}=0.55$ (0.52-0.59); $\mathrm{SC}=0.36(0.34-0.39)$; $\mathrm{FB}=1.44$ (1.37-1.54); BL = 2.91 (2.79-3.09). Habitus as in Fig. 85. Forebody appear moderately shining because of rather light colouration and strong sculpture, abdomen very finely transverse coriaceous microsculptured and punctate with large but shallow punctures, yet shinier than forebody. Body slightly reddish medium brown, except clypeal area, explanate sides of pronotum, legs, mouthparts and antennomeres 1-4 light to medium brown, two blackish spots at the bases of supraantennal prominences near circular clypeal impression and two similar dark spots at hind vertex. Head with anterior margin possessing thin rim, evenly arched between supraantennal prominences but at the latter (elevated oblique ridges) more narrowly rounded, disc impressed near eyes. Epistomal suture forming subcircular impression (inside unsculptured), upper surface without tactile setae, eyes small and rather bulging; in dorsal view temples (1.8× length of eye) alatiform, laterally rather angled, posteriorly constricted (truncate) to form distinct neck, however, dorsal side not separated by occipital furrow, rather by dorsum of neck bearing microsculpture instead of punctation. Antennomere 1 stout club-shaped and smooth, segment 2 ovoid, segment 3 club-shaped, segment 4 small and isodiametricalal, segments $5-11$ with basal dishes, articles 6-10 slightly transverse (1.0-1.2× broader than long). Head and pronotum with mostly foveate sculpture. Pronotum with lateral portion explanate, edge with a few short setae, anterior corners broadly rounded, lateral margin almost evenly arched, hind margin before corners insignificantly concave; disc medially with a pair of shinier parallel longitudinal ridges, partly impressed alongside. Elytra with very thin membranous posterior margin extending from sutural corner to 3/4 of hind margin. Elytral epipleural ridge present with up-turned lateral alatiform portions, disc depressed; shoulders moderately developed, surface foveolate, in between punctures indistinctly microsculptured. Legs short, pro- and mesotibia with several spinulose rows, metatibia with longitudinal ctenidium of spinules in distal half. Abdomen with sides moderately arcuate, second segment with paratergites strongly broadening posteriorly (abdomen appearing constricted at base), those of segments III-VII thin, mesal paratergites broad; posterior edge of tergite VII with thin palisade fringe, median part of sternite VII as in Fig. 22, sternite VIII (Fig. 23), tergites IX (Fig. 24), X (Fig. 25) and aedeagus as in Figs 26-28.

\footnotetext{
Distribution - Only known from Darjeeling in India and the immediately neighbouring area of Nepal.

Remarks - No type material was made available for study, nevertheless, the description compared to abundant material from the type locality leaves no doubt about the correct interpretation of the taxon.
} 


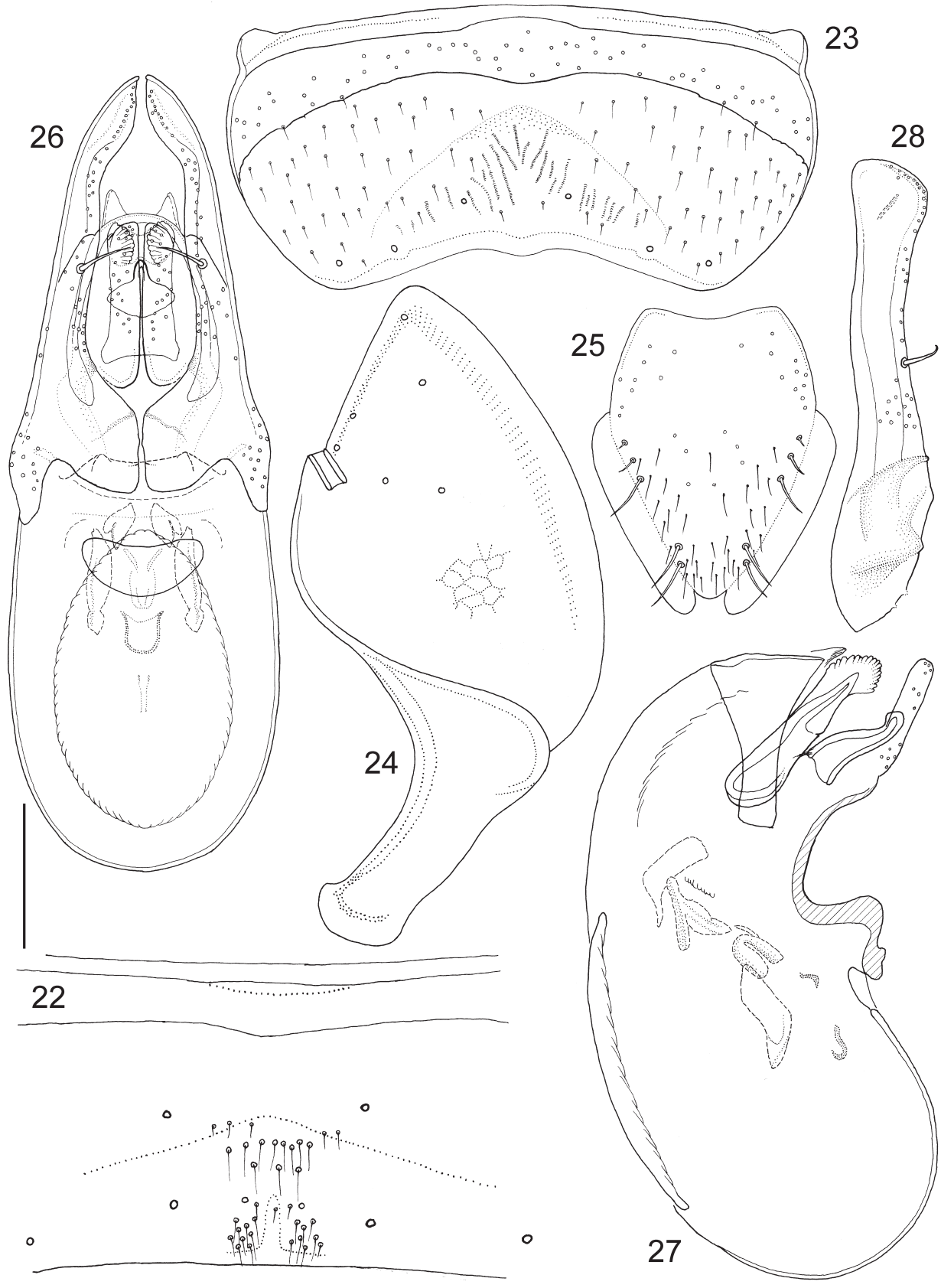

Figs 22-28. Anotylus bengalensis (Biswas et Sengupta, 1980), male. 22 = median part of sternite VII, 23 = sternite VIII, 24 = tergite IX, 25 = tergite $X, 26$ = aedeagus, frontal view, $27=$ median lobe, lateral view, 28 = paramere, lateral view. Scales: $0.10 \mathrm{~mm}$ for Figs 26-28, 0.115 $\mathrm{mm}$ for Fig. 22, $0.13 \mathrm{~mm}$ for Figs 24-25, $0.14 \mathrm{~mm}$ for Fig. 23 


\section{Anotylus borneensis (Cameron, 1928)}

(Figs 29-35, 74)

Oxytelopsis borneensis Cameron, 1928: 401; Herman 1970: 413; Hammond 1984: 200, HerMAN 2001: 1413.

Examined type material - Oxytelopsis borneensis Cameron - Holotype (†): “Type [red margined disc, curator label] \Mt Pais [=Poi, $\left.01^{\circ} 48^{\prime} \mathrm{N}, 109^{\circ} 41^{\prime} \mathrm{E}\right]$; Borneo $\backslash$ Type; Oxytelopsis; borneensis; Dr. Cameron \M. Cameron.; Bequest.; B.M. 1955-147." (BMNH).

Other material - MALAYSIA/BORNEO: Sabah, Sepilok [Forest Reserve, 0551'N, $118^{\circ} 00^{\prime}$ E, near Sandakan], IV-V. 1982, leg. M. Horák (1 O', MHNG); Sabah, Sapulut env., ca. 100 km E Keningau, 23.VI.1998, leg. F. Čiampor \& J. Kodada (1 + , NHMW); Sabah, Mt. Kinabalu National Park Headquarters, 1560-1660 m, river Silau Silau, 24.IV.1987, leg. A. Smetana (B2), sifting of old flood debris (1 $\left.\delta^{\lambda}, \mathrm{FMNH}\right)$; Sabah, Crocker Range N. P., NW Keningau, 900-1200 m, 18.XI.1996, leg. D. Grimm, at light (1 + , SMNS).

Redescription - Measurements (in mm, n=5): HW $=0.55(0.51-0.60)$; TW $=0.52(0.49-$ $0.58) ; \mathrm{PW}=0.73(0.68-0.80) ; \mathrm{EW}=0.83(0.75-0.90) ; \mathrm{AW}=0.82(0.75-0.88) ; \mathrm{HL}=0.39(0.38-$ $0.42) ; \mathrm{EL}=0.13(0.12-0.14) ; \mathrm{TL}=0.16(0.15-0.17) ; \mathrm{PL}=0.45$ (0.43-0.49); $\mathrm{SC}=0.54(0.49-0.60)$; $\mathrm{FB}=1.43$ (1.36-1.58); $\mathrm{BL}=2.66$ (2.48-2.88). Habitus as in Fig. 74. Forebody weakly shining despite sculpture, abdomen with punctures of varying sizes, rather dense but shallow, finely and indistinctly microsculptured at places, yet more lustrous than forebody. Head dark brown, pronotum and abdomen plus legs reddish medium to dark brown, elytra occasionally lighter, medium brown around posterior suture towards shoulders. Mouthparts and antennae reddish medium brown. Head with anterior margin possessing rather strong rim, almost truncate in front, laterally straight to supraantennal prominences (moderately elevated oblique ridges), disc slightly impressed near eyes. Epistomal suture forming subcircular impression (much less punctate inside), upper surface with weak tactile setae (intratemporalis) situated postero-medially of each eye; eyes medium sized and slightly bulging, in dorsal view temples (1.2× length of eye) alatiform, laterally evenly rounded and posteriorly constricted to form distinct neck separated by weak occipital furrow. Antennomere 1 flattened club-shaped, strongly sculptured, segment 2 basally broad subtriangular, segment 3 incrassate, segment 4 transverse discoid with rim, segments 5-11 with basal dishes, articles 5-10 transverse (about 1.5-1.7× broader than long). Head with areolate-rugulose sculpture, pronotum alveolate. Pronotum with lateral portion explanate, edge alatiform with sparse short setae; lateral margin unevenly arched with a number of small, irregularly distributed denticles, posterior corners not incised, disc medially with two weak parallel longitudinal ridges, pronotal sides weakly impressed around middle. Elytra without membranous posterior margin. Elytral epipleural ridge apparent as stronger sculpture lines in lateral alatiform portions, disc with very slight to no impression in centre; shoulders well developed, surface obscurely foveolate and in between punctures torulose. Legs short, pro- and mesotibia with several spinulose rows, metatibia with longitudinal ctenidium of spinules in distal half. Abdomen with sides moderately/rather arcuate, second segment with paratergites broadening posteriorly (abdomen appearing constricted at base), those of segments III-VII thin, mesal paratergites broad, posterior edge of tergite VII with thin palisade fringe, median part of sternite VII as in Fig. 29, sternite VIII (Fig. 30), tergites IX (Fig. 31), X (Fig. 32) and aedeagus as in Figs 33-35. 


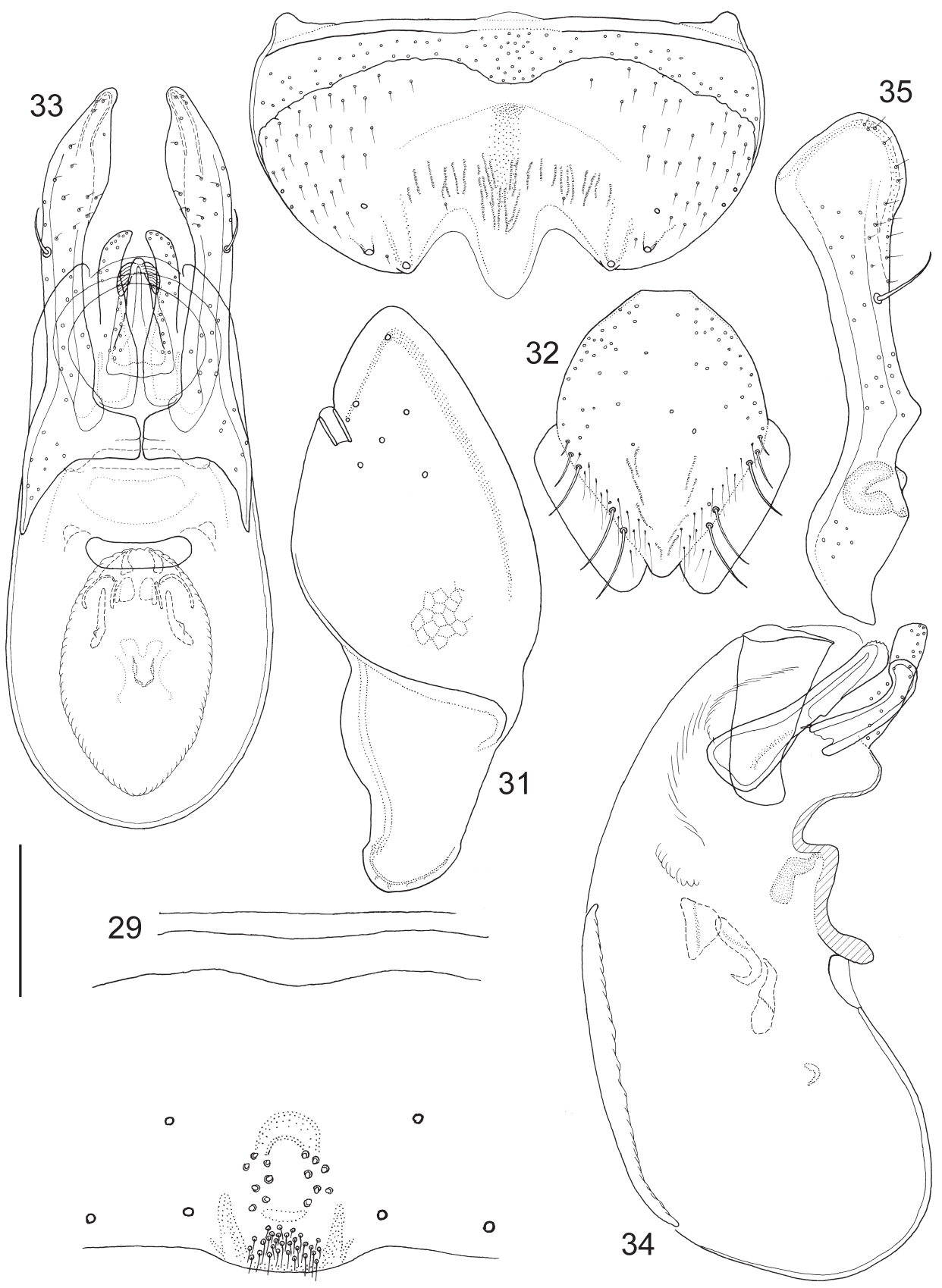

Figs 29-35. Anotylus borneensis (Cameron, 1928), male. 29 = median part of sternite VII, $30=$ sternite VIII, $31=$ tergite IX, 32 = tergite $X, 33$ = aedeagus, frontal view, $34=$ median lobe, lateral view, 35 = paramere, lateral view. Scales: $0.10 \mathrm{~mm}$ for Figs 32-35, $0.12 \mathrm{~mm}$ for Figs 29, 31, $0.15 \mathrm{~mm}$ for Fig. 30 
Distribution - Besides the holotype (at that locality a single specimen was collected), only a couple of exemplars have become subsequently known, all from Sabah (N Borneo).

Remarks - The holotype is from Mt. Poi, undoubtedly a rare species. The only specimen for which bionomics is recorded was found by sifting of old flood debris.

\section{Anotylus ceylonicus (Hammond, 1975) \\ (Figs 36-42, 80)}

Oxytelopsis ceylonicus Hammond, 1975: 170; Herman 2001: 1413.

Examined type material - Oxytelopsis ceylonicus Hammond - Holotype (ð): “Holo-; type [red margined disc] \Ceylan [Central]; Hakgala 28.I.[19]70; 1700-1800 m; Mussard Besuchet Löbl; [on the back, in pencil] 30c [tamisages dans la forêt vierge au-dessus du jardin botanique, à $1800 \mathrm{~m}] \backslash$ Oxytelopsis; ceylonicus sp.n.; P.M. Hammond; det. 1971; Holotype ${ }^{1 \prime \prime}$ (MHNG); Paratypes (29): same data as holotype (1 +, MHNG); SRI LANKA: Central prov., Horton Plains, 12 mi SSE Nuwara-Eliya, 7000 ft, 19.III.1962, leg. Brinck, Andersson, Cederholm - Lund University Ceylon Expedition (163), jungle, sieved in debris (3

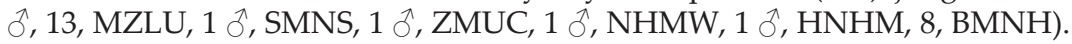

Redescription - Measurements (in mm, $\mathrm{n}=10)$ : $\mathrm{HW}=0.65(0.60-0.69) ; \mathrm{TW}=0.62(0.57-$ $0.68) ; \mathrm{PW}=0.84(0.79-0.88) ; \mathrm{EW}=0.88(0.83-0.95) ; \mathrm{AW}=0.96(0.90-1.03) ; \mathrm{HL}=0.53(0.49-$ $0.57) ; \mathrm{EL}=0.13(0.12-0.14) ; \mathrm{TL}=0.21(0.19-0.23) ; \mathrm{PL}=0.52(0.47-0.56) ; \mathrm{SC}=0.40(0.36-0.42)$; FB = 1.51 (1.39-1.59); BL = 3.40 (2.96-3.84). Habitus as in Fig. 80. Forebody somewhat shining despite sculpture, abdomen on segments II-VI without (so shinier than forebody), but segments VII-X with coriaceous microsculpture, former roughly punctured with indistinct borders, latter with less conspicuous punctation, less lustrous. Forebody reddish dark brown, supraantennal area and explanate part of pronotum lighter, abdomen blackish dark brown. Mouthparts and antennomeres 1-4 reddish medium brown, rest of antennomeres plus legs reddish medium to dark brown. Head with anterior margin possessing extremely thin rim, somewhat unevenly arched, anterior clypeal rim very slightly projecting in middle, supraantennal prominences as moderately elevated oblique ridges, disc impressed near eyes. Epistomal suture forming circular impression (inside finer punctured), upper surface without tactile setae, eyes small and slightly bulging; in dorsal view temples (1.6× length of eye) moderately alatiform, laterally weakly rounded (somewhat angled) and posteriorly constricted to form distinct neck, however, dorsal side not separated by occipital furrow, rather by dorsum of neck bearing microsculpture instead of punctation. Antennomere 1 club-shaped and rather smooth, segment 2 ovoid, segment 3 club-shaped, segment 4 small and isodiametrical, segments 5-11 with basal dishes, articles 6-10 transverse (about $1.8 \times$ broader than long), segments $9-10$ bulkier than preceding. Head and pronotum with mostly lacunose sculpture. Pronotum with lateral portion explanate and slightly up-turned, edge with sparse short setae, lateral border with several rather prominent angles, gradually transforming into smaller teeth posteriorly, hind margin before corners with shallow incision; disc medially rather convex, parallel longitudinal ridges rather obscured, laterally broadly impressed along sides. Elytra without membranous posterior margin (completely reduced). Elytral epipleural ridge present with up-turned lateral 


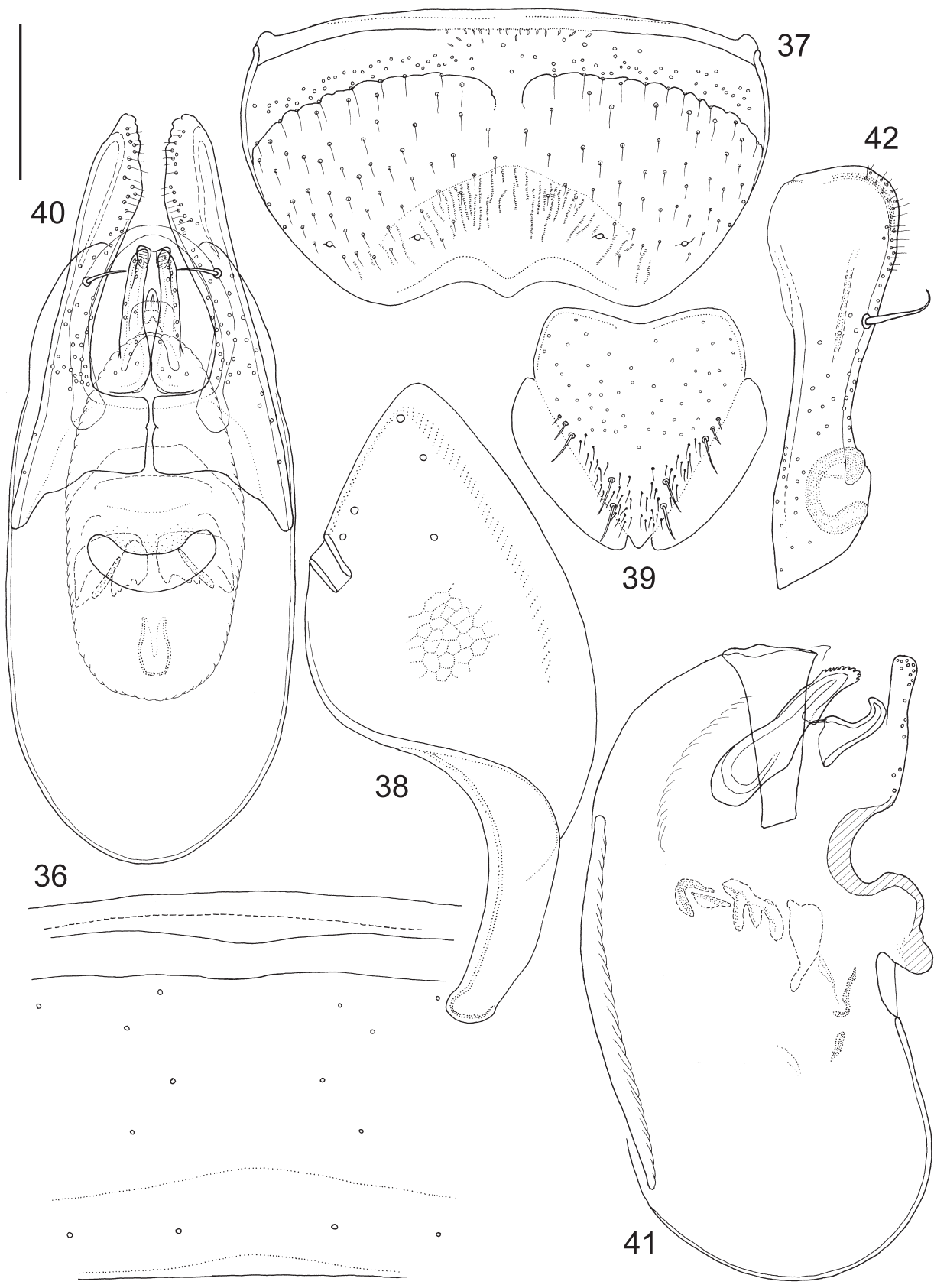

Figs 36-42. Anotylus ceylonicus (Hammond, 1975), male. $36=$ median part of sternite VII, 37 = sternite VIII, 38 = tergite IX, $39=$ tergite $X, 40=$ aedeagus, frontal view, $41=$ median lobe, lateral view, 42 = paramere, lateral view. Scales: $0.10 \mathrm{~mm}$ for Figs $40-42,0.14 \mathrm{~mm}$ for Fig. 38, $0.155 \mathrm{~mm}$ for Figs 36, 39, $0.17 \mathrm{~mm}$ for Fig. 37 
alatiform portions, disc depressed; shoulders moderately developed, surface foveolate, in between punctures indistinctly sculptured. Legs short, pro- and mesotibia with several spinulose rows, metatibia with longitudinal ctenidium of spinules in distal half in distal $2 / 3$. Abdomen with sides very weakly arcuate, second segment with paratergites strongly broadening posteriorly (abdomen appearing constricted at base), those of segments III-VII thin, mesal paratergites broad; posterior edge of tergite VII with thin palisade fringe, median part of sternite VII as in Fig. 36, sternite VIII (Fig. 37), tergites IX (Fig. 380), X (Fig. 39) and aedeagus as in Figs 40-42.

\title{
Distribution - The species is only known from the higher altitude areas of Ceylon (Sri Lanka).
}

Remarks - No more material has become known since the description.

\author{
Anotylus chinensis (Bernhauer, 1938) \\ (Figs 43-49, 78)
}

Oxytelopsis chinensis Bernhauer, 1938: 20; Herman 1970: 413; Herman 2001: 1413.

Oxytelopsis shibatai Ito, 1987: 75, syn. nov.; Herman 2001: 1415.

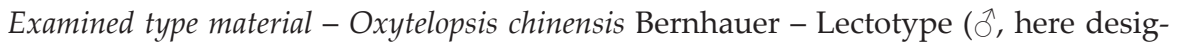
nated): "Para-; lecto-; type [light blue margined disc, curator label] $\backslash$ Para-; type [yellow margined disc, curator label] \Cotypus \Nordwestl. [sic] China; Chinkiang [sic] [see remark]; Col. Reitter \Oxytelopsis; chinensis; Brnh. Cotyp. \Brit. Mus; 1946-126. \Oxytelopsis; chinensis Bernh.; P.M. Hammond; det. 1970; Paralectotype [sic] \Anotylus; chinensis (Bernhauer); det. Makranczy, 2015" (BMNH), Paralectotype (): "Nordwestl. China; Chinkiang; Col. Reitter \Oxytelopsis; chinensis; Brnh. Typ. \ chinensis; Brnh. Typus; Oxytelopsis \Chicago NHMus; M. Bernhauer; Collection \ Lecto-; type [lilac margined disc, curator label] \Oxytelopsis; chinensis Bnh; P.M. Hammond; det. 1974; Lectotype [sic] $q \backslash$ FMNH-INS; 0000063473 \Photographed; D. Le 2011; EMu catalog" (FMNH), Paralectotype (+): "Para-; lecto-; type [light blue margined disc, curator label] \ Nordwestl. China; Chinkiang; Col. Reitter \ chinensis; Brnh. Cotypus; Oxytelopsis \Chicago NHMus; M. Bernhauer; Collection \Oxytelopsis; chinensis Bnh.; P.M. Hammond; det. 1974 \&; Paralectotype \Syntype; teste D. J. Clarke; GDI Imaging Project" (FMNH); Oxytelopsis shibatai Ito - Paratype (ठ): "Imoyama; Yamato; 2.VI.1984; Ito \Paratype [red card] \Oxytelopsis; shibatai; T. Ito; Det. Tateo Ito, 1987” (coll. Ito), Paratype (): “Imoyama; Yamato; 2.VI.1984; Ito \Paratype [red card] \Oxytelopsis; shibatai; T. Ito; Det. Tateo Ito, 1987" (coll. Ito).

Other material - CHINA: Sichuan, W-, Ya'an prefecture, Fulin Co., Daxiang Ling, road between Hanyuanjie and Siping, $51 \mathrm{~km}$ NNE Shimian, 29 $39^{\prime} \mathrm{N}, 102^{\circ} 37^{\prime} \mathrm{E}, 2300 \mathrm{~m}$, 10.VII.1999, leg. M. Schülke, Ufer, Gesiebe (1 + , coll. Schülke); Jiangxi prov., Jinggangshan Mts., Xiangzhou (forested valley $\mathrm{S}$ of the village), $26^{\circ} 35.5^{\prime} \mathrm{N}, 114^{\circ} 16.0^{\prime} \mathrm{E}, 374 \mathrm{~m}, 26 . \mathrm{IV} .2011$, leg. M. Fikaček \& J. Hájek (MF08), accumulation of moist leaf litter along the stream and on the steep slope above the stream in the sparse secondary forest, sifting $(1 \hat{0}, 1 q \mathrm{NMPC}, 1$ + , SMNS); INDIA: Meghalaya, Khasi Hills, en-dessus de Pynursla, 1100 m, 29.X.1978, leg. C. Besuchet \& I. Löbl (34), tamisage en forêt, dans un petit ravin (1 §̊, MHNG).

Redescription - Measurements (in $\mathrm{mm}, \mathrm{n}=7)$ : HW $=0.66(0.62-0.72)$; $\mathrm{TW}=0.64(0.59$ $0.70) ; \mathrm{PW}=0.90$ (0.85-0.99); $\mathrm{EW}=1.00$ (0.91-1.09); $\mathrm{AW}=1.03$ (0.95-1.10); $\mathrm{HL}=0.51$ (0.48$0.56) ; \mathrm{EL}=0.14(0.13-0.15) ; \mathrm{TL}=0.21(0.20-0.23) ; \mathrm{PL}=0.57(0.53-0.62) ; \mathrm{SC}=0.66(0.59-0.71)$; 


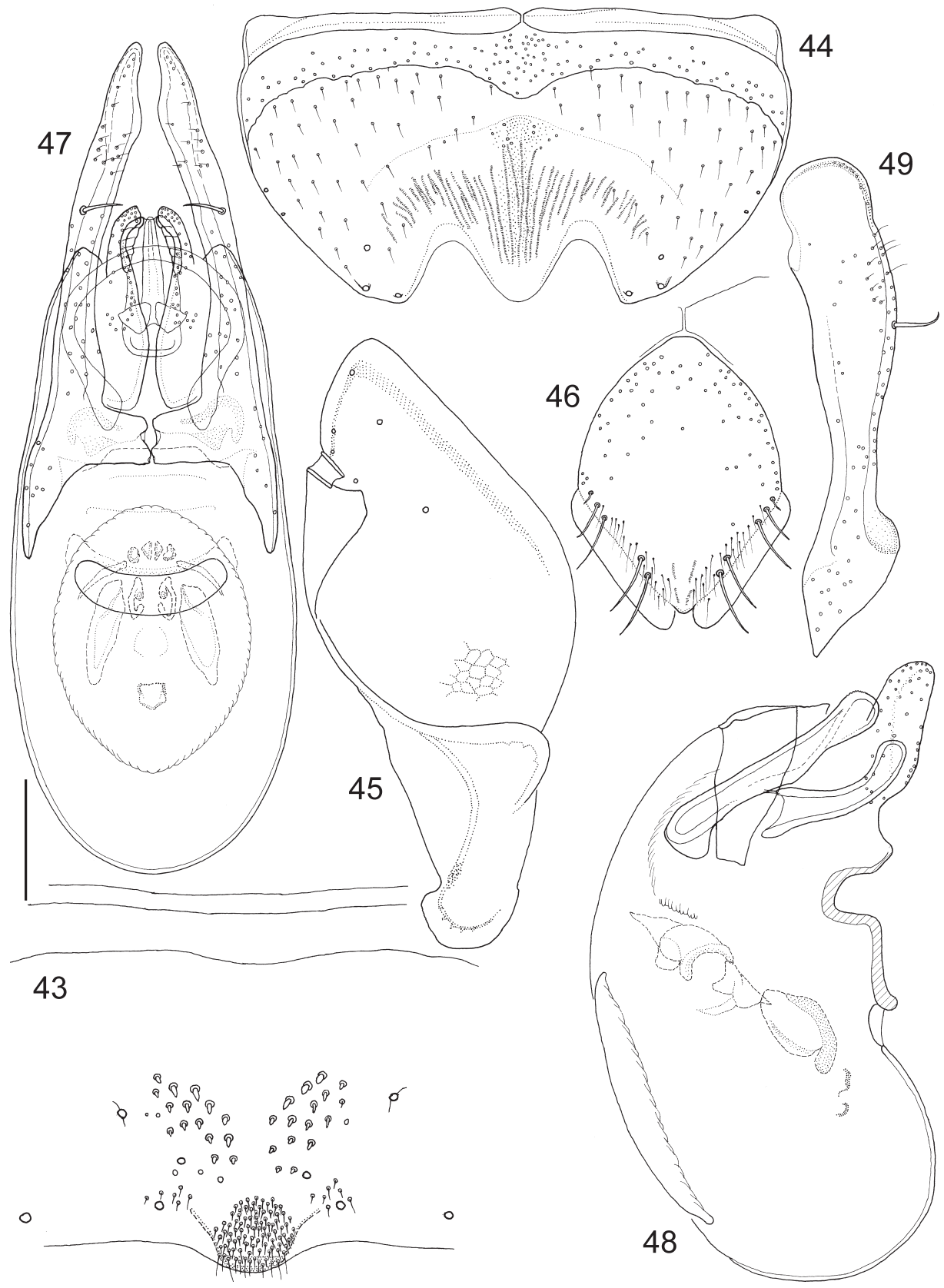

Figs 43-49. Anotylus chinensis (Bernhauer, 1938), male. $43=$ median part of sternite VII, $44=$ sternite VIII, $45=$ tergite IX, 46 = tergite $X, 47$ = aedeagus, frontal view, $48=$ median lobe, lateral view, 49 = paramere, lateral view. Scales: $0.10 \mathrm{~mm}$ for Figs $47-49,0.11 \mathrm{~mm}$ for Figs 43, 46, $0.14 \mathrm{~mm}$ for Figs 44-45 
FB $=1.80$ (1.69-1.95); BL = 3.50 (3.25-3.81). Habitus as in Fig. 78. Forebody weakly shining because of sculpture, abdomen very finely microsculptured, finely and loosely punctured, somewhat more lustrous. Body reddish medium to dark brown, head (except clypeal area) often darker, blackish dark brown, middle of tergite bases often blackish as well as most of antennomere 1. Head with anterior margin possessing rim (weaker in middle), evenly arched but slightly angled at supraantennal prominences, these as moderately elevated oblique ridges, rim continuing posteriad along inner border of eyes, disc slightly impressed beside it. Epistomal suture forming circular impression (less sculptured inside), upper surface with weak tactile setae (intratemporalis) situated postero-medially of each eye. Eyes medium-sized and slightly bulging, in dorsal view temples (1.5× length of eye) alatiform, laterally rounded and posteriorly constricted to form distinct neck, separated by transversal, weak occipital furrow. Antennomere 1 flattened club-shaped and strongly microsculptured, segment 2 oval but broad at base, segment 3 club-shaped, segment 4 small and slightly transverse, segments 5-11 with basal dishes, articles 6-10 transverse (about $1.5 \times$ broader than long), apex of segment 11 lighter. Head with areolate-rugose, pronotum lacunose sculpture. Pronotum with lateral portion explanate and slightly up-turned, edge alatiform with very sparse short setae; lateral border unevenly arched with thin marginal ridge and a number of small, irregularly distributed denticles, posterior margin evenly arched; disc medially with two nearly parallel and posteriorly confluent longitudinal ridges, posteriorly impressed alongside them, laterally somewhat impressed towards side margin. Elytra with membranous posterior margin extending from sutural corner to 3/4 of hind margin. Elytral epipleural ridge apparent as stronger sculpture lines in lateral alatiform portions, disc with very slight to no impression in centre; shoulders well developed, surface obscurely foveolate and in between punctures torulose. Legs short, pro- and mesotibia with several spinulose rows, metatibia with longitudinal ctenidium of spinules in distal half. Abdomen with sides moderately arcuate, second segment with paratergites broadening posteriorly (abdomen slightly constricted at base), those of segments III-VII thin, mesal paratergites broad; posterior edge of tergite VII with thin palisade fringe, median part of sternite VII as in Fig. 43, sternite VIII (Fig. 44), tergites IX (Fig. 45), X (Fig. 46) and aedeagus as in Figs 47-49.

Distribution - The species is known from several localities in Japan (see Іто 2007).

Remarks - The locality "Chin-kiang, NW China" is not precise. The real locality is now considered to be in Jiangsu not Xinjiang and could be Zhenjiang; similar fauna to those from this locality is known from other places in Jiangsu. P. Hammond's labelling of the type specimens of "Oxytelopsis chinensis" is very strange; regardless of what might have caused this erroneous action, the male syntype must be chosen as lectotype of $O$. chinensis. A habitus photo of "Oxytelopsis shibatai" can be found in Iто (2007: 173, fig. 1).

\section{Anotylus cimicoides (Fauvel, 1895)}

$$
\text { (Figs 50-56, 100) }
$$

Oxytelopsis cimicoides Fauvel, 1895: 200; Cameron 1930: 205; Herman 1970: 413; Coiffait 1984: 150, Herman 2001: 1413.

Oxytelopsis taiwana Ito, 1987: 77, syn. nov.; Herman 2001: 1415. 
Examined type material - Oxytelopsis cimicoides Fauvel - Lectotype ( $\partial$, here designated): “Lecto-; type [lilac margined disc, curator label] \[Mt.] Carin; Asciuii Ghecù [19 $17^{\circ} \mathrm{N}$, 96² 2 `. $^{\prime}$ E]; 1400-1500 m.; L. Fea. III-IV.[18]88. \ cimicoides; Fvl. \Coll. et det. A. Fauvel; Oxytelopsis; cimicoides Fauv.; R.I.Sc.N.B. $17.479 \backslash$ G. Fagel det., 195; Anotylopsis; gen. nov. \Syntype \Oxytelopsis; cimicoides Fvl.; P.M. Hammond; det. 1972; Lectotype \ $\hat{\sigma}$ $\backslash$ Anotylus; cimicoides (Fauvel); det. Makranczy, 2015" (ISNB); Paralectotypes (3): "Para-; lecto-; type [light blue margined disc] \Carin; Asciuii Ghecù; 1400-1500 m.; L. Fea. III-IV. [18]88. \ Coll. et det. A. Fauvel; Oxytelopsis; cimicoides Fauv.; R.I.Sc.N.B. 17.479 \G. Fagel det., 19; Anotylopsis; gen. nov. \Syntype \Oxytelopsis; cimicoides Fvl.; P.M. Hammond; det. 1972; Paralectotype \Anotylus; cimicoides (Fauvel); det. Makranczy, 2015" (2 §̃, 1 ㅇ, ISNB); Oxytelopsis taiwana Ito - Paratype (ठ): “Funchiifo; Taiwan; 24.III.1970; T. Kobayashi [on yellowish card] \Paratype [red card] \Oxytelopsis; taiwanus; T. Ito; Det. Tateo Ito, 1987" (coll. Ito), Paratype (+): "Mt. Yangming; Taiwan; 30.IV.1982; T. Ito [on yellowish card] \Paratype [red card] \Oxytelopsis; taiwanus; T. Ito; Det. Tateo Ito, 1987" (coll. Ito).

Other material - PAKISTAN: Punjab, Murree, 2100 m, 05.VI.1983, leg. C. Besuchet \& I. Löbl (40), tamisage de feuilles mortes dans une forêt de pins et feuilles (4, MHNG); $\mathrm{N}$-, Punjab, Murree, 1950 m, 23.IV.1984, leg. S. Vít, litière du Quercus (1, MHNG); NEPAL: E-, Rolwaling Himal, Tama Koshi Tal, N Chetchet, 16.V.2000, leg. A. Kleeberg (1 , coll. Kleeberg); Tama Koshi valley, unterh. Simigaon, 1300 m, 03.VI.2000, leg. A. Kleeberg (1 q, coll. Kleeberg); Tama Koshi valley, Chet Chet, 1300 m, 03.VI.2000, leg. J. Schmidt (2 ô, coll.

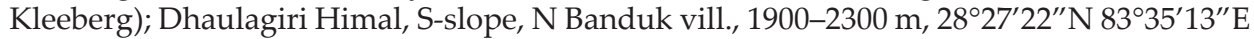
to $28^{\circ} 28^{\prime} 07^{\prime \prime} \mathrm{N} 83^{\circ} 35^{\prime} 10^{\prime \prime} \mathrm{E}, 06 . V .2009$, leg. J. Schmidt (1, NKME); Manaslu Mts., S of Bara Pokhari, 2300 m, 08.IV.2003, leg. J. Schmidt (26, NKME); Manaslu Mts., S of Bara Pokhari, 2300 m, 07.IV.2003, leg. J. Schmidt (3, NKME); Manaslu Mts., S Bara Pokhari, ca. $28^{\circ} 15^{\prime} \mathrm{N}$, $84^{\circ} 25^{\prime}$ E, 2100 m, 29.IV.2005, leg. J. Schmidt (6, NKME); Manaslu Mts., Dudh Pokhari Lekh below Helam Pokhari, 2000 m, 22.IV.2003, leg. J. Schmidt, sifting forest litter (1, NKME); Lalitpur distr., 2 km S Godavari, 1700 m, 12.IX.1983, leg. A. Smetana \& I. Löbl (1), en amont d'un ruisseau, ravin boisé, tamisage de feuilles mortes et de bois pourri (2, MHNG); Lalitpur distr., 2 km S Godavari, 1700 m, 20.X.1983, leg. A. Smetana \& I. Löbl (39), ravin boisé, tamisage de feuilles mortes (9, MHNG, 1 ऽ, HNHM); distr. Kathmandu, Godawari, 1600 $\mathrm{m}$, forêt au-dessus du jardin botanique, 31.III.1984, leg. I. Löbl (1), tamisage de feuilles mortes près d'un ruisseau, humide (1, MHNG); prov. Bagmati, Phulchauki au sud de Kathmandu, 1700 m, 10.V.1981, leg. I. Löbl (67), tamisage de feuilles mortes dans un ravin boisé (2, MHNG); INDIA: Kumaon (Uttar Pradesh = Uttarakhand), Bhim Tal, env. 1500 m, 4.X.1979, leg. I. Löbl (1), lisière d'une forêt secondaire, versant est, assez sec, tamisage (1, MHNG); Kumaon (Uttar Pradesh), Rangarh, env. 2000 m, ravin boisé, 9.X.1979, leg. I. Löbl (6b), tamisage des feuilles mortes (40, MHNG); Kumaon (Uttar Pradesh), Ramgarh [29 $\left.25^{`} \mathrm{~N}, 7^{\circ} 33^{`} \mathrm{E}\right], 2250 \mathrm{~m}$, leg. 9.X.1979, leg. I. Löbl (7), crête; tamisage au pied de rhododendrons, assez sec (4, MHNG); Kumaon (Uttar Pradesh), Chaubattia près Ranikhet

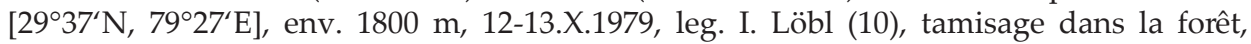
feuilles mortes, fougères et mousses (8, MHNG); Kumaon (Uttar Pradesh), Chaubattia près Ranikhet, ravin, 1950 m, 14.X.1979, leg. I. Löbl (11), l’herbe et feuilles mortes au bord d'un ruisseau, tamisage (4, MHNG); Garhwal (Uttar Pradesh), au-dessus de Mussoorie, $1700 \mathrm{~m}$, versant sud, ravin dans la foret, 19.X.1979, leg. I. Löbl (15), tamisage, assez humide (8, MHNG); Meghalaya, Garo Hills, au-dessus de Tura, 700-900 m, forêt du Tura Peak, 1. XI.1978, leg. C. Besuchet \& I. Löbl (37b), tamisage en forêt (4, MHNG); Meghalaya, West Garo Hills distr., trail Tura - Tura Peak summit, $25^{\circ} 30^{\prime} 28^{\prime \prime} \mathrm{N}, 90^{\circ} 13^{\prime} 54^{\prime \prime} \mathrm{E}, 650 \mathrm{~m}$, 14.X.2004, leg. G. Cuccodoro, C. Carlton, R. Leschen \& D. Errne (2a), sifting leaf litter in forest near stream (2, MHNG); Meghalaya, West Garo Hills distr., trail Tura - Tura Peak summit, aban- 
doned upper portion of trail, $25^{\circ} 30^{\prime} 12^{\prime \prime} \mathrm{N}, 90^{\circ} 14^{\prime} 07^{\prime \prime} \mathrm{E}, 800 \mathrm{~m}, 14 . X .2004$, leg. G. Cuccodoro, C. Carlton, R. Leschen \& D. Errne (2b), sifting leaf litter in forest (8, MHNG); Meghalaya, 1 $\mathrm{km}$ E of Tura, 300-600 m, $25^{\circ} 30^{\prime} \mathrm{N}, 90^{\circ} 14^{`} \mathrm{E}, 2-5 . V .2002$, leg. M. Trýzna \& P. Benda (3, coll. Schülke); West Bengal, Darjeeling distr., Sukna, 200 m, 7.X.1978, leg. C. Besuchet, I. Löbl (2b), tamisage en forêt (1, MHNG); N-, Assam, Umrongso env. 700 m, $25^{\circ} 27^{`} \mathrm{~N}, 92^{\circ} 43^{`} \mathrm{E}, 3-8$. VI.2002, leg. M. Trýzna \& P. Benda (3, coll. Schülke); NE-, Assam/Arunachal Pr. border, Bhalukpong, $27^{\circ} 00^{\prime} 48^{\prime \prime} \mathrm{N}, 92^{\circ} 39^{\prime} 08^{\prime \prime} \mathrm{E}, 150 \mathrm{~m}, 1-8 . V .2012$, leg. L. Dembický, sifting + Winkler app. extraction (1, ZFMK); NE-, Arunachal Pr., Etalin vicinity, $28^{\circ} 36^{\prime} 56^{\prime \prime} \mathrm{N}, 9^{\circ} 53^{\prime} 21^{\prime \prime} \mathrm{E}, 700$ m, 15-25.V.2012, leg. L. Dembický, sifting + Winkler app. extraction (7, ZFMK, 1, HNHM); MYANMAR: Shan State, ca. $35 \mathrm{~km}$ N Aungban, Mintaingbin Forest Camp, primary riverine forest, extending ca. 50-100 m from Mintaingbin river, peripheric area cultivated, $20^{\circ} 55.20^{\prime} \mathrm{N}, 96^{\circ} 33.60^{\prime} \mathrm{E}$, ca. $1320 \mathrm{~m}, 11-23 . \mathrm{VI} .2004$, leg. H. Schillhammer, H. Shaverdo, \& U Myint Hlaing (146a), FIT (1, NHMW); Mandalay Reg., Mogok Township, S Panlin vill., Mt. Taung Mae, west slope, 1710-1750 m, ca. 22 $58^{\prime} 09^{\prime \prime} \mathrm{N}, 9^{\circ} 27^{\prime} 13^{\prime \prime} \mathrm{E}, 10 .-18 . V I .2014$, leg. A. Brunke \& H. Schillhammer (MBS 201a), sifted (4, NHMW); Mandalay Region, ca. 7 km NW Mogok, above road Mogok-Bernardmyo, S Panlin village, west slope of Mt. Taung Mae, ca. $22^{\circ} 58^{\prime} 05.8^{\prime \prime} \mathrm{N}, 9^{\circ} 27^{\prime} 29^{\prime \prime} \mathrm{E}$, ca. $1870 \mathrm{~m}, 17-24$.V.2016, leg. H. Schillhammer, A. Brunke, J. Jenkins Shaw, A. Jensen (212b), slightly disturbed primary forest, sifting of leaf litter (1 NHMW); CHINA: Sichuan, S-, env. Xichang, 1600 m, 28.VII.1996, leg. S. Kurbatov, litter (3, MHNG); Sichuan, S-, Jinping Shan, E slope, Luning env., 1600 m, 14-20.VI.2004, leg. R. Fabbri, mixed subtropical shrubs (1, coll. Schülke); Sichuan, S-, Xichang, monastery, 1800-1900 m, 17-20.VI.2004, leg. R. Fabbri, primary deciduous forest (1, coll. Schülke); Sichuan, Qingcheng Shan, Rückseite, 3053`56“N, 103³3`01“E, 650-700 m, 18.V.1997, leg. M. Schülke (2), small remaining spots of subtropical broadleaved forest and field edges, sifted from litter and moss (25, coll. Schülke), same but 30 $53^{\prime} 57^{\prime \prime} \mathrm{N}, 103^{\circ} 32^{\prime} 23^{\prime \prime} \mathrm{E}, 3-4$.VI.1997, (17) (2, coll. Schülke); Sichuan, C-, Wenjiang distr., Dujiangyan Co., Qingcheng Shan, 56 km NW Chengdu, 3054'N, 1033'ㄹ, 975 m, 18.VI.1999, leg. M. Schülke, Bachufer, Waldrest (21, coll. Schülke), same but Unkrauthaufen (3, coll. Schülke); Yunnan, E Kunming, Xiaobailong Forest Park, $24^{\circ} 55^{\prime} 43^{\prime \prime} \mathrm{N}, 103^{\circ} 05^{\prime} 22^{\prime} \mathrm{E}, 2110 \mathrm{~m}, 10$. VIII.2014, leg. V. Assing (3), secondary pine forest, pine litter and litter at trail margin sifted (18, coll. Assing); Yunnan, NE Kunming, secondary pine forest, with scattered old alder, $25^{\circ} 09^{\prime} 07^{\prime \prime} \mathrm{N}, 102^{\circ} 53^{\prime} 46^{\prime \prime} \mathrm{E}, 2280$ $\mathrm{m}$, 11.VIII.2014, leg. V. Assing (4), leaf litter, sifted (13, coll. Assing); Yunnan, NE Kunming, mixed forest, $2^{\circ} 08^{\prime} 35^{\prime \prime} \mathrm{N}, 102^{\circ} 53^{\prime} 49^{\prime \prime} \mathrm{E}, 2320 \mathrm{~m}, 13 . \mathrm{VIII} .2014$, leg. V. Assing (6), mixed forest with alder, oak, and pine, litter and mushrooms sifted (1, coll. Assing); Yunnan, mountain

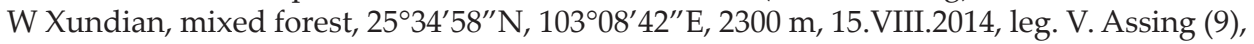
mixed forest with alder, pine, shrub undergrowth, litter, twigs, and roots of herbs sifted (2, coll. Assing); Yunnan, mountain NW Wuding, mixed forest, $25^{\circ} 36^{\prime} 53^{\prime \prime} \mathrm{N}, 102^{\circ} 18^{\prime} 59^{\prime \prime} \mathrm{E}, 2190$ $\mathrm{m}, 17$. VIII.2014, leg. V. Assing (10), degraded mixed forest with alder, oak, and pine, litter, mushrooms, and dead wood sifted (11, coll. Assing); Yunnan, Wuding, Lion Mountain, $25^{\circ} 31^{\prime} 59^{\prime \prime} \mathrm{N}, 102^{\circ} 22^{\prime} 36^{\prime \prime} \mathrm{E}, 2200 \mathrm{~m}, 17 . \mathrm{VIII} .2014$, leg. V. Assing (11), stream valley with deciduous forest, moist litter sifted (14, coll. Assing); Yunnan, mountain WNW Wuding, mixed forest, $25^{\circ} 38^{\prime} 45^{\prime \prime} \mathrm{N}, 102^{\circ} 06^{\prime} 55^{\prime \prime} \mathrm{E}, 2390 \mathrm{~m}$, 18.VIII.2014, leg. V. Assing (12), mixed forest margin with alder and pine, litter sifted (1, coll. Assing); Yunnan, mountain SE Gejiu, graveyard with pine, $23^{\circ} 18^{\prime} 27^{\prime \prime} \mathrm{N}, 103^{\circ} 11^{\prime} 41^{\prime \prime} \mathrm{E}, 2400 \mathrm{~m}, 20 . \mathrm{VIII} .2014$, leg. V. Assing (13), pine litter and herb roots sifted (3, coll. Assing); Yunnan, valley S Gejiu, road margin, $23^{\circ} 08^{\prime} 38^{\prime \prime} \mathrm{N}, 103^{\circ} 11^{\prime} 42^{\prime \prime} \mathrm{E}, 1010 \mathrm{~m}, 21$.VIII.2014, leg. V. Assing (15), sifted from litter and soil (1, coll. Assing); Yunnan, mountains S Jianshui, 2325'20”N, 102 $51^{\prime} 05^{\prime \prime} \mathrm{E}, 1890 \mathrm{~m}, 22$. VIII.2014, leg. V. Assing (18), subtropical broad-leaved forest, litter sifted (6, coll. Assing); 


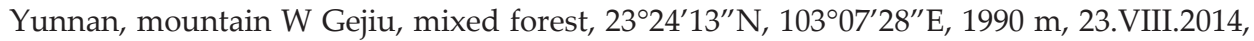
leg. V. Assing (20), litter and various debris sifted (10, coll. Assing); Yunnan, SE Pingbian, Dawei Shan, 22 $54^{\prime} 31^{\prime \prime} \mathrm{N}, 103^{\circ} 41^{\prime} 44^{\prime \prime} \mathrm{E}, 2100 \mathrm{~m}, 27 . \mathrm{VIII} .2014$, leg. V. Assing (22), primary subtropical broad-leaved forest, litter sifted (3, coll. Assing); Yunnan, mountain W Yuxi, secondary mixed forest, $24^{\circ} 27^{\prime} 11^{\prime \prime} \mathrm{N}, 102^{\circ} 29^{\prime} 58^{\prime \prime} \mathrm{E}, 2250 \mathrm{~m}, 31 . \mathrm{VIII} .2014$, leg. V. Assing (23), litter, roots, and moss sifted (1, coll. Assing); Yunnan, N-, Lijiang Naxi Auton. Co., 3 km NW Yongsheng, $53 \mathrm{~km}$ WSW Lijiang, SE slope, $26^{\circ} 41.8^{\prime} \mathrm{N}, 100^{\circ} 43.1^{\prime} \mathrm{E}, 1950-2000 \mathrm{~m}$, 14.VIII.2003, leg. M. Schülke (C03-03), secondary broadleaved forest (13, coll. Schülke); Yunnan prov., Shanzi env., Jizu Shan Mt., 2756.8’ N, 100²3.9’ E, 2000 m, 22-24.VI.2007, leg. J. Hájek \& J. Růžička (CH44), sifted detritus and leaves near trunk bases, dense mixed forest (with dominant Pinus, Quercus and Rhododendron) near stream (1, coll. Schülke); Yunnan prov., Shanzi env., Jizu Shan Mt., along path to summit, $27^{\circ} 57.7-8^{\prime} \mathrm{N}, 100^{\circ} 22.1$ 23.6'E, 2180-2580 m, 22-24.VI.2007, leg. J. Hájek \& J. Růžička (CH45-47), sifted detritus and leaves, dense mixed (wet) forest (dominant Pinus, Quercus and Rhododendron) near stream (1, coll. Schülke); Zhejiang, Tianmu Shan, pass $25 \mathrm{~km} \mathrm{NNW}$ Linan, $30^{\circ} 25^{\prime} 40^{\prime \prime} \mathrm{N}, 119^{\circ} 35^{\prime} 30^{\prime \prime} \mathrm{E}$, 620-820 m, 16.VI.2007, leg. D.W. Wrase (37), creek valley, bamboo, mixed forest, litter, sifted (1, coll. Schülke); CHINA/TAIWAN: Taipei Co., Beitou township, TianMu Park, 30.XII.2008, leg. S. Vít (1), compost (34, MHNG); Taipei Co., Beitou township, Jiantan metro station, Jiantan Shan Hiking Trail, 02.I.2009, leg. S. Vít (2), dead stump and bark (12, MHNG), same but (3), decaying bole (5, MHNG), same but (4), dead tree trunk and bark (7, MHNG), same but (5), dead stump and bark (16, MHNG); Taipei Co., Beitou township, Shipai metro station, Mt. Samau (S), TianMu Gudao Hiking Trail, 3.I.2009, leg. S. Vít (6), upper layer of forest litter (12, MHNG), same but (7) old forest litter (4, MHNG), same but (8) hollow tree base (2, MHNG); Taipei Co., Beitou township, Shipai metro station, Veteran Hospital, Junjian Yan Hiking Trail, 6.I.2009, leg. S. Vít (9), fern litter (1, MHNG), same but (10) dead stump (4, MHNG); Chiayi Co., Alishan Natural Scenic Area, Road 18, km 84, 2200 m, 7.I.2009, leg. S. Vít (12), sifting forest litter (92, MHNG), same but 8.I.2009 (14) decaying stump (resinferous) (8, MHNG); Miaoli Co., Nanjhuang township, E-Nanjhuang, Road 21, km 3, 25.X.2010, leg. S. Vít (1), forest litter (19, MHNG); Miaoli Co., Nanjhuang township, E-Nanjhuang, Road 21, km 2, 25.X.2010, leg. S. Vít (2), upper stratum of gully litter (2, MHNG); Miaoli Co., Nanjhuang township, E-Nanjhuang, Road 124, km 37, 26.X.2010, leg. S. Vít (5), putres. bundle (1, MHNG); Taoyuan Co., DaSi township, Shihmen Reservoir Area, After Bay Hilly Side, 25.XI.2010, leg. S. Vít (9), deep gully litter (13, MHNG), Kaohsiung Co., Shanping Work Station, $22^{\circ} 58.040^{\prime} \mathrm{N}, 120^{\circ} 41.143^{\prime} \mathrm{E}, 823 \mathrm{~m}, 28 . \mathrm{IX} .2007$, leg. D. Rédei \& J.-F. Tsai (9), swept (1, HNHM), Kaohsiung Hsien, near Liukuei, Shanping LTER Site, 1.IV.2003, leg. L. Papp \& M. Földvári, swept (1 万人, HNHM); Pingtung Co., 10km E of Mutan, 400 m, 7-8.IV.1997, leg. G. Csorba \& L. Ronkay, at light (1, HNHM); THAILAND: Chiang Mai prov., Doi Suthep, versant nord, 1550 m, 4.XI.1985, leg. D. Burckhardt \& I. Löbl (8), tamisage de feuilles mortes dans un petit ravin avec ruisselet (6, MHNG); Chiang Mai prov., Doi Suthep, versant nord, 1050 m, 5.XI.1985, leg. D. Burckhardt \& I. Löbl (10), ravin très humide, tamisage de bois pourri de écorces et champignons (1, MHNG); Chiang Mai prov., Doi Suthep, versant nord, 1400 m, 5.XI.1985, leg. D. Burckhardt \& I. Löbl (11), ravin très humide, tamisage de feuilles mortes près d'un ruisseau (17, MHNG); Doi Suthep, Tham Ryssie, 1150 m, 2.XI.1996, leg. P. Schwendinger (1, MHNG); Phetchaburi prov., Kaeng Krachan National Park, 450 m, à 35 km de "Headquarters", 19.XI.1985, leg. D. Burckhardt \& I. Löbl (26), tamisage de débris végétaux dans la forêt (1, MHNG); Chanthaburi prov., Khao Sabap Nat. Park, environs de Phliu Waterfalls, 150-300 m, 23-24. XI.1985, leg. D. Burckhardt \& I. Löbl (27a), tamisage de débris végétaux et de mousses à 
proximité de cours d'eau (1, MHNG); Nakhon Ratchasima prov., NE Bangkok, Khao Yai Nat. Park, aux environs de "Headquarters", 750-850 m, 26.XI.-3.XII.1985, leg. D. Burckhardt \& I. Löbl (28b), tamisage de débris végétaux (21, MHNG, 1 ð, HNHM); MALAYSIA:

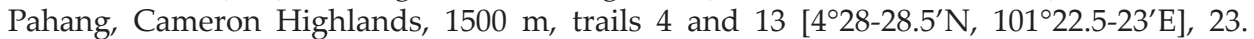
III.1993, leg. I. Löbl \& F. Calame (15), tamisage de feuilles mortes et de racines près d'un tronc (3, MHNG); Pahang, Cameron Highlands, Gunung Jasar, trail 11, $1550 \mathrm{~m}\left[4^{\circ} 28^{\prime 2} 28^{\prime \prime} \mathrm{N}\right.$, 101²1'47"E], 24.III.1993, leg. I. Löbl \& F. Calame (18b), tamisage de feuilles mortes (7, MHNG); Pahang, Cameron Highlands, Tanah Rata, 4300 ft, 7.VIII.1972, leg. T. Jacoud (6, MHNG); Ringlet, 1250 m, 26.III.1993, leg. I. Löbl \& F. Calame (20), tamisage de feuilles pourries et du bois dans un ravin et à la lisière d'un forêt dégradées (3, MHNG); prov. Pahang, Cameron Highlands, Gunung Jasar, ca. 1500 m, 24-25.I.1992 leg. H. Schillhammer (5c), sifted, Junglewalk Nr. 11, small stream, 0.5-0.7 m wide, partly flowing underground, (1 $\widehat{\jmath}$, NHMW), Pahang, Cameron Highlands, Tahah Rata, Gunung Jasar, 1400-1500 m, 2025.I.1995, leg. S. Bečváŕ jr. \& sn. (1, NMPC); Pahang, Cameron Highlands, Tanah Rata, edge of degraded rainforest, 21.III.-2.IV.1995, leg. O. Merkl (72), at light (10, HNHM); Pahang, Cameron Highlands, 2 km S Tanah Rata on Tapah Road, 29.III.1995, leg. O. Merkl \& I. Szikossy (93), at light (2, HNHM); Perak, Maxwell Hill, 1100 m, 21.XI.1999, leg. G. Cuccodoro \& I. Löbl (11a), evergreen broadleaf montane rainforest, vegetable refutes around guesthouse (9, MHNG), same but (11b), forest leaf litter and vegetational debris along road (2, MHNG); Perak, Maxwell Hill, 950 m, 22.XI.1999, leg. G. Cuccodoro \& I. Löbl (12), evergreen broadleaf montane rainforest, forest leaf litter and vegetational debris (1, MHNG); Perak, Maxwell Hill, Gunung Hijau, near summit, 1250 m, 23.XI.1999, leg. G. Cuccodoro \& I. Löbl (13), evergreen broadleaf montane rainforest, forest leaf litter and vegetational debris (4, MHNG); Perak, Maxwell Hill, 1150 m, 24.XI.1999, leg. G. Cuccodoro \& I. Löbl (14c), evergreen broadleaf montane rainforest, vegetational debris along road (5, MHNG), same but 25.XI.1999 (15a) (4, MHNG); PHILIPPINES/MINDANAO: Mt. Agtuuganon, 1050 m, 28.V-7.VI.1996, leg. Mey (1 ô, 1 + , ZMHB).

Redescription - Measurements (in mm, $\mathrm{n}=10)$ : $\mathrm{HW}=0.69(0.62-0.75)$; $\mathrm{TW}=0.71(0.63-$ $0.79) ; \mathrm{PW}=1.01$ (0.90-1.09); $\mathrm{EW}=1.14$ (1.02-1.25); $\mathrm{AW}=1.10$ (0.97-1.22); $\mathrm{HL}=0.53$ (0.48$0.57) ; \mathrm{EL}=0.15$ (0.14-0.16); $\mathrm{TL}=0.20$ (0.18-0.21); $\mathrm{PL}=0.59$ (0.53-0.65); $\mathrm{SC}=0.69$ (0.60-0.77); $\mathrm{FB}=1.87(1.68-2.04) ; \mathrm{BL}=3.44(3.05-3.76)$. Habitus as in Fig. 100. Forebody moderately shining despite sculpture, abdomen very finely microsculptured and shallowly punctured, less lustrous. Body reddish dark brown but clypeal area lighter, reddish (abdomen occasionally also lighter); mouthparts and antennae medium brown. Head with anterior margin forming an arch more or less truncate in middle, an extremely thin rim, supraantennal prominences as moderately elevated oblique ridges, disc slightly impressed near eyes, middle of vertex slightly elevated. Epistomal suture forming subcircular impression (almost unpunctured inside), upper surface without tactile setae, eyes medium large, moderately convex; in dorsal view temples (1.3× length of eye) alatiform, laterally angled or more rounded and posteriorly constricted to form distinct neck, however, dorsal side not separated by occipital furrow, rather by dorsum of neck bearing microsculpture instead of punctation. Antennomere 1 flattened club-shaped and finely microsculptured, segment 2 oval, segment 3 club-shaped, segment 4 almost round, segments $5-11$ with basal dishes, segments 6-10 transverse (almost $2 \times$ broader than long). Head and pronotum with mostly lacunose sculpture. Pronotum with lateral portion explanate and slightly up-turned, edge alatiform with sparse short setae; lateral border uneven and with a number of small, irregularly distributed denticles, posterior margin incised before corners; disc medially with two parallel and posteriorly almost confluent longitudinal ridges, posteriorly impressed 


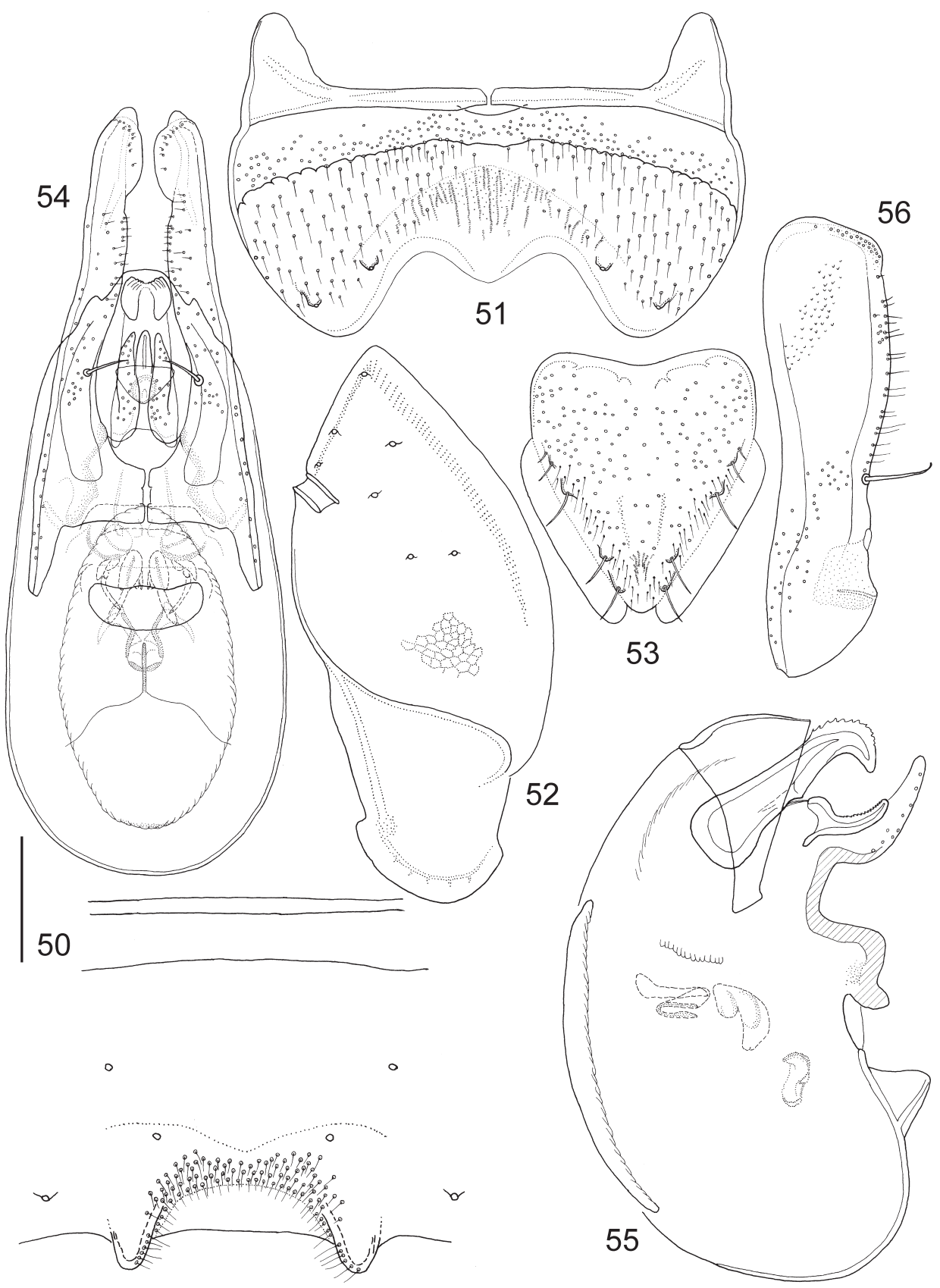

Figs 50-56. Anotylus cimicoides (Fauvel, 1895), male. $50=$ median part of sternite VII, $51=$ sternite VIII, $52=$ tergite IX, 53 = tergite $X, 54=$ aedeagus, frontal view, $55=$ median lobe, lateral view, 56 = paramere, lateral view. Scales: $0.10 \mathrm{~mm}$ for Figs 54-56, $0.13 \mathrm{~mm}$ for Figs 50, 53, $0.14 \mathrm{~mm}$ for Fig. 52, $0.16 \mathrm{~mm}$ for Fig. 51 
alongside them, laterally impressed around middle of sides. Elytra with membranous posterior margin extending from sutural corner to $4 / 5$ of hind margin. Elytral epipleural ridge present with up-turned lateral alatiform portions, disc longitudinally impressed alongside; shoulders well developed, surface foveolate, in between punctures torulose. Legs short, pro- and mesotibia with several spinulose rows, metatibia with longitudinal ctenidium of spinules in distal half. Abdomen with sides gently arcuate, second segment with paratergites strongly broadening posteriorly (abdomen appearing constricted at base), those of segments III-VII thin, mesal paratergites broad; posterior edge of tergite VII with thin palisade fringe, median part of sternite VII as in Fig. 50, sternite VIII (Fig. 51), tergites IX (Fig. 52), X (Fig. 53) and aedeagus as in Figs 54-56.

Distribution - The distribution ranges from the whole Himalaya all the way to peninsular Malaysia, southern China and the Philippines.

Remarks - The confusing external similarity to A. excisicollis and the great variability makes most previous records of this species rather unreliable.

\section{Anotylus coonoor sp. n.}

(Figs 57-63, 76, 265)

Type material - Holotype (đ̋): INDIA: Madras [=Tamil Nadu], Nilgiri, Hulical près de Coonoor $\left[11^{\circ} 20^{\prime} \mathrm{N}, 76^{\circ} 46^{\prime} \mathrm{E}\right]$, sur la rive droite de Coonoor river, $1600 \mathrm{~m}$, 22.XI.1972, leg. C. Besuchet, I. Löbl \& R. Mussard (44), tamisages en forêt, dans un ravin (MHNG); Paratypes (2): same data as holotype (1 3 , NHMW); Madras, Nilgiri, $6 \mathrm{~km}$ E Coonoor, $1400 \mathrm{~m}$, 22.XI.1972, leg. C. Besuchet, I. Löbl \& R. Mussard (42), tamisages en forêt près d'une rivière (1, MHNG).

Description - Measurements (in $\mathrm{mm}, \mathrm{n}=3)$ : $\mathrm{HW}=0.76(0.73-0.78) ; \mathrm{TW}=0.80(0.76-$ $0.83) ; \mathrm{PW}=1.07$ (0.99-1.14); $\mathrm{EW}=1.13$ (1.09-1.18); $\mathrm{AW}=1.19$ (1.13-1.25); $\mathrm{HL}=0.59$ (0.56$0.62) ; \mathrm{EL}=0.15(0.14-0.16) ; \mathrm{TL}=0.23(0.20-0.25) ; \mathrm{PL}=0.62(0.59-0.64) ; \mathrm{SC}=0.66(0.62-0.70)$; $\mathrm{FB}=1.96(1.87-2.01) ; \mathrm{BL}=3.81$ (3.68-4.00). Habitus as in Fig. 76. Forebody rather dull for dense sculpture, abdomen densely punctured and weakly microsculptured, more lustrous. Head and first antennomeres blackish dark brown, rest of body reddish dark brown, anterior margin of head often lighter, reddish. Head with anterior margin possessing marked rim (weaker in middle), evenly arched but slightly angled at supraantennal prominences, these as moderately elevated oblique ridges, rim continuing posteriad along inner border of eyes, disc slightly impressed beside it. Epistomal suture forming obsolete circular impression (sculpture inside not differing), upper surface with tactile setae (intratemporalis) situated postero-medially of each eye. Eyes medium-sized and slightly bulging, in dorsal view temples (1.5× length of eye) alatiform, laterally rounded and posteriorly constricted to form distinct neck, separated by transversal occipital furrow. Antennomere 1 flattened club-shaped and strongly microsculptured, segment 2 basally broad ovoid, segment 3 clubshaped, segment 4 small and slightly transverse, segments 5-11 with basal dishes, articles 6-10 transverse (about 1.4 $\times$ broader than long). Head and pronotum with lacunose sculpture, in midline slightly transitional to costulate. Pronotum with lateral portion explanate, edge alatiform with very sparse short setae; lateral border almost evenly arched with thin marginal ridge and a number of small, irregularly distributed denticles, posterior margin evenly arched, posterior corners inconspicuous; disc medially with two posteriorly confluent longitudinal ridges, slightly impressed alongside them, laterally somewhat impressed 


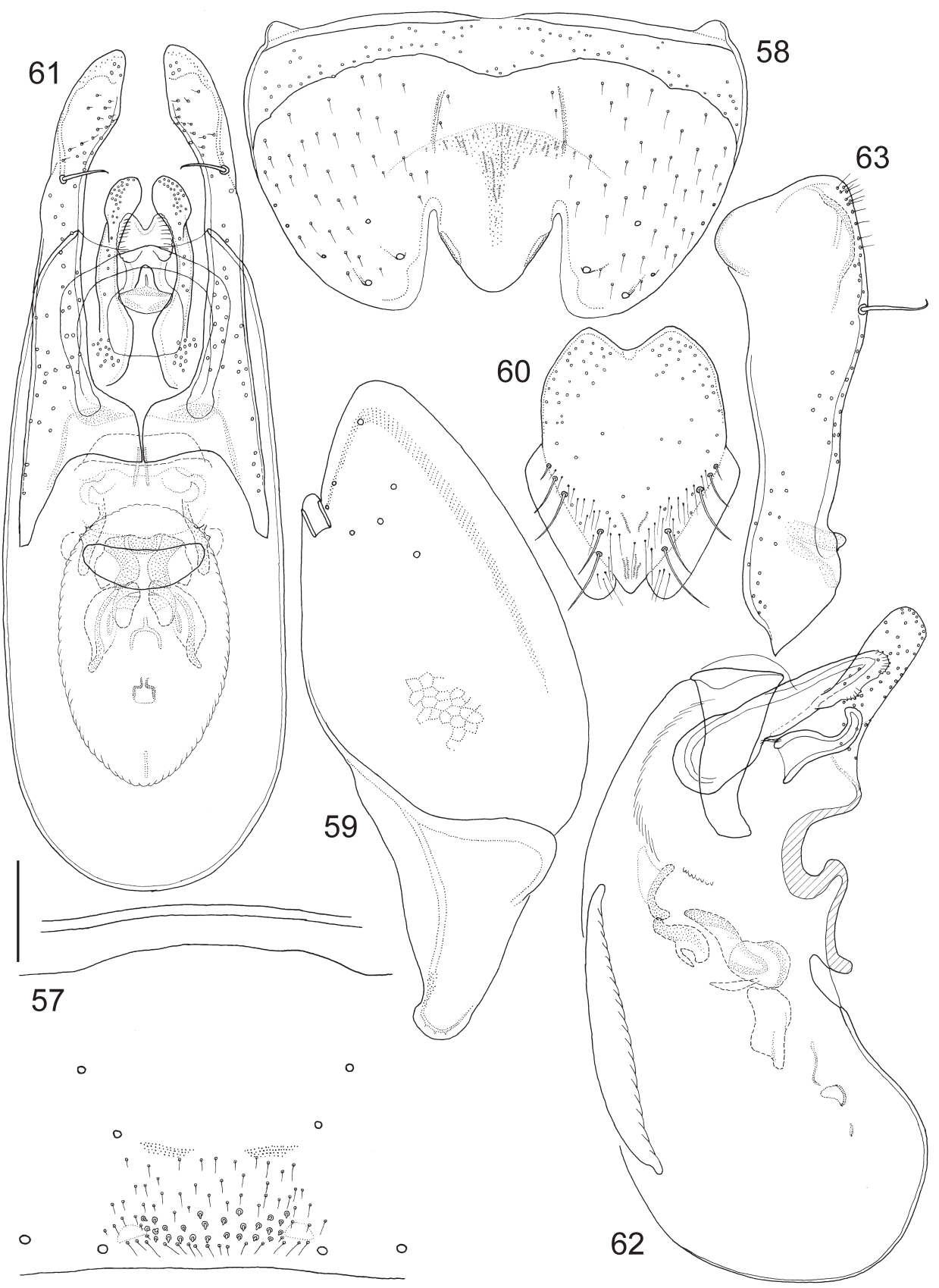

Figs 57-63. Anotylus coonoor sp. n., male. $57=$ median part of sternite VII, $58=$ sternite VIII, 59 = tergite IX, 60 = tergite $X, 61$ = aedeagus, frontal view, 62 = median lobe, lateral view, 63 = paramere, lateral view. Scales: $0.10 \mathrm{~mm}$ for Figs 61-63, $0.115 \mathrm{~mm}$ for Figs 57, 59-60, $0.13 \mathrm{~mm}$ for Fig. 58 
near side margin. Elytra without membranous posterior margin. Elytral epipleural ridge apparent as stronger sculpture lines in lateral alatiform portions, disc with very slight to no impression in centre; shoulders well developed, surface obscurely foveolate, elevations of interspaces conflent as short longitudinal ridges. Legs short, pro- and mesotibia with several spinulose rows, metatibia with longitudinal ctenidium of spinules in distal half. Abdomen with sides moderately arcuate, second segment with paratergites broadening posteriorly (abdomen slightly constricted at base), those of segments III-VII thin, mesal paratergites broad; posterior edge of tergite VII with thin palisade fringe, median part of sternite VII as in Fig. 57, sternite VIII (Fig. 58), tergites IX (Fig. 59), X (Fig. 60) and aedeagus as in Figs 61-63, spermatheca as in Fig. 265.

Etymology - The name derives from the populated place Coonoor near the type locality (noun in apposition).

Distribution - Only known from a small area in southern India.

Remarks - This species is most closely related to A. gunung, both with rather conspicuous, dark, stout and erect tactile setae on head and pronotum.

\section{Anotylus excisicollis (Bernhauer, 1938) \\ (Figs 64-70, 101)}

Oxytelopsis excisicollis Bernhauer, 1938: 21; Herman 1970: 413; Herman 2001: 1413.

Examined type material - Oxytelopsis excisicollis Bernhauer - Holotype ( + ): “Nitou Tatsienlu [=Kangding Co.]; Szechuan China; Em.[merich] Reitter \ Reitteri Brh.; det. Bernhau[er] \excisicollis; Brnh. Typus; Un.[icus] Oxytelopsis \Chicago NHMus; M. Bernhauer; Collection \Holo-; type [red margined disc, curator label] \Oxytelopsis; excisicollis Bnh; P.M. Hammond; det. 1974; Holotype $ᄋ$ \FMNH-INS; $0000063474 \backslash$ Photographed; D. Le 2011; EMu catalog" (FMNH).

Other material - INDIA: West Bengal, Darjeeling distr., Mahanadi près de Kurseong, versant sud, 1200 m, 6.X.1978, leg. C. Besuchet \& I. Löbl (1), tamisage en forêt (17, MHNG); West Bengal, Darjeeling distr., entre Algarah et Labha, à $7 \mathrm{~km}$ d'Algarah, versant sud, 1900 m, 11.X.1978, leg. C. Besuchet \& I. Löbl (10), tamisage en forêt (4, MHNG); West Bengal, Darjeeling distr., entre Ghoom et Lopchu, à $13 \mathrm{~km}$ de Ghoom, versant nord, $2000 \mathrm{~m}$, 12.X.1978, leg. C. Besuchet \& I. Löbl (12), tamisage en forêt (1, MHNG); West Bengal, Darjeeling distr., Algarah, 1800 m, 9.X.1978, leg. C. Besuchet \& I. Löbl (6), tamisage en forêt (9, MHNG); West Bengal, Darjeeling distr., entre Algarah et Labha, à 7 km d'Algarah, versant sud, 1900 m, 11.X.1978, leg. C. Besuchet \& I. Löbl (10), tamisage en forêt (12, MHNG); West Bengal, Darjeeling distr., Mahanadi près de Kurseong, versant sud, 1200 m, 19.X.1978, leg. C. Besuchet \& I. Löbl (20), tamisage en forêt (2, MHNG); Meghalaya, Khasi Hills, au-dessus de Shillong, N-slope, 1850-1950 m, forêt primaire du Shillong Peak, 25.X.1978, leg. C. Besuchet \& I. Löbl (27), tamisage en forêt (3, MHNG); Meghalaya, Khasi Hills, en-dessus de Cherrapunjee, 1200 m, 26.X.1978, leg. C. Besuchet \& I. Löbl (28b), tamisage en forêt, au pied de rochers (14, MHNG); Meghalaya, Khasi Hills, ente Mawsynram et Balat, à $16 \mathrm{~km}$ de Mawsynram, 1000 m, 27.X.1978, leg. C. Besuchet \& I. Löbl (30b), tamisage en forêt, dans un

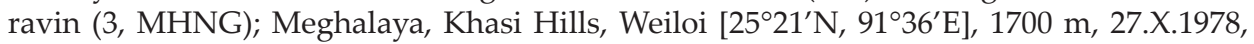


leg. C. Besuchet \& I. Löbl (31), tamisage en forêt (2, MHNG); Meghalaya, West Garo Hills distr., Mt. Nokrek National Park, trail Tura peak - Mt. Nokrek, $25^{\circ} 30^{\prime} 02^{\prime \prime} \mathrm{N}, 90^{\circ} 14^{\prime} 54^{\prime \prime} \mathrm{E}$, 1200 m, 17.X.2004, leg. G. Cuccodoro, C. Carlton, R. Leschen \& D. Errne (5a), sifting broadleaf litter in forest (7, MHNG); Meghalaya, West Garo Hills distr., Tura Peak, near summit, $25^{\circ} 30^{\prime} 16^{\prime \prime} \mathrm{N}, 90^{\circ} 14^{\prime} 38^{\prime \prime} \mathrm{E}, 1200$ m, 17.X.2004, leg. G. Cuccodoro, C. Carlton, R. Leschen \& D. Errne (5b), sifting leaf litter and vegetational debris at base of big tree in forest (2, MHNG);

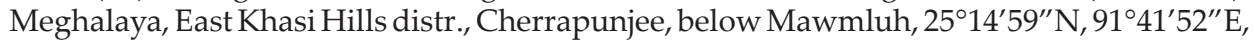
1200 m, 24.X.2004, leg. G. Cuccodoro, C. Carlton, R. Leschen \& D. Errne (12a), in forest, sifting broadleaf litter (1, MHNG); Assam, North Cachar Hills distr., North Cachar Hills,

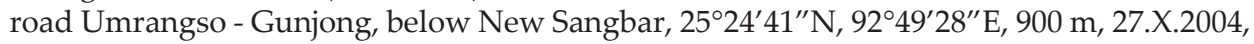
leg. G. Cuccodoro, C. Carlton, R. Leschen \& D. Errne (15b), sifting leaf litter and flood debris in dry bed of temporary stream in patch of mixed Quercus forest (2, MHNG); NEPAL: prov. Bagmati, Dobate Ridge NE Barahbise, 2700 m, 2.V.1981, leg. I. Löbl \& A. Smetana (54), tamisage de feuilles mortes et de mousses dans une chênaie (2 ô, MHNG); Vallée d'Arun au-dessous de Num, près du pont principal, 1100 m, ravin boisé latéral, leg. I. Löbl \& A. Smetana (34), tamisage de feuilles mortes accumulées sur les roches (1 q, MHNG); Vallée d'Arun au-dessous de Num, près du pont principal, $1050 \mathrm{~m}$, forêt hygrophile à palmiers, 22.IV.1984, leg. I. Löbl \& A. Smetana (35c), tamisage de feuilles mortes et bois pourri (1 +, MHNG); Manaslu Mts., S of Bara Pokhari, 2000 m, 7.IV.2003, leg. J. Schmidt (2, NKME); Manaslu Mts., S of Bara Pokhari, 2300 m, 8.IV.2003, leg. J. Schmidt (9, NKME, 1 ô, HNHM); Manaslu Mts., S of Bara Pokhari, ca. $28^{\circ} 15^{`} \mathrm{~N}, 84^{\circ} 25^{`} \mathrm{E}, 2100$ m, 29.IV.2005, leg. J. Schmidt (1, NKME); MYANMAR: Sagaing Division, Alaungdaw Katthapa NP, ca. 1.5 km SW Log Cabin Camp, along Pagoda Road, $22^{\circ} 18.902^{\prime}$ N 94²8.060'E, ca. 400 m, 6.V.2003, leg. H. Schillhammer et al. (108), semi-evergreen forest close to Pagoda Stream; sifting of moist leaf litter in small ravines (2 $\widehat{\partial}, 5, \mathrm{NHMW})$; Sagaing Division, Alaungdaw Katthapa NP, around Ku Mara monastery, $22^{\circ} 18.560^{\prime} \mathrm{N}, 9^{\circ} 27.679^{\prime} \mathrm{E}$, ca. 400 m, 8.V.2003, leg. H. Schillhammer et al. (117), sifting of moist leaf litter among stones in dried-up stream-bed, shaded by large bushes (11, NHMW, 1 ڤึ, HNHM); CHINA: Sichuan, S-, Ya'an Prefecture, Shimian Co., Xiaoxiang Ling, side-valley above Nanya Cun near Caluo, $11 \mathrm{~km}$ S Shimian, ca. 1250 m, 7.VII.1999, leg. M Schülke, Rinde, Pilze, Streu (1 §, 2 \& , 2, coll. Schülke), same but leg. D. W. Wrase (5, coll. Schülke, 1 స, HNHM); Yunnan, Dali Bai Auton. Pref., Wuliang

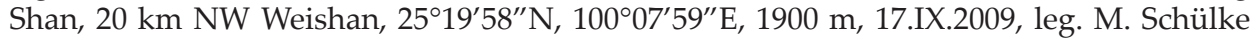
(CH09-58), creek valley, litter \& old flood debris sifted (1, coll. Schülke); Yunnan, Gaoligong Shan, pass SW Baoshan, 4-6.VI.2006, leg. I. Jeniš (1 $\hat{\jmath}$, coll. Assing, $1 \hat{\jmath}$, HNHM); Yunnan, Baoshan Pref., Gaoligong Shan, nr. Xiaoheishan N. R., 35 km SE Tengchong, $24^{\circ} 50^{\prime} 16^{\prime \prime} \mathrm{N}, 98^{\circ} 45^{\prime} 43^{\prime \prime} \mathrm{E}, 2110 \mathrm{~m}, 30 . \mathrm{V} / 4 . \mathrm{VI} .2007$, leg. D.W. Wrase (11), primary decid. forest, litter, sifted (3, coll. Schülke); Yunnan, Baoshan Prefecture, Gaoligong Shan, E pass,

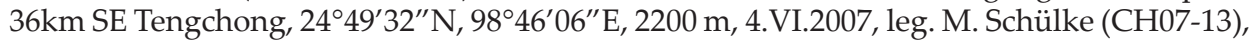
deciduous forest, litter, wood, fungi sifted (2, coll. Schülke); Yunnan, Baoshan Pref., mountain range $14 \mathrm{~km}$ E Tengchong, $25^{\circ} 00^{\prime} 28^{\prime \prime} \mathrm{N}, 98^{\circ} 38^{\prime} 07^{\prime \prime} \mathrm{E}, 1850 \mathrm{~m}, 1 . \mathrm{VI} .2007$, leg. D.W. Wrase (16), secondary mixed forest, field edge, litter, debris sifted (10, coll. Schülke); Yunnan, Baoshan Pref., Gaoligong Shan, W pass 32 km SE Tengchong, 2451'11"N, 9844'27' E, 1600 m, 28.VIII.2009, leg. M. Schülke (CH09-14), cleft with devastated primary forest, litter and mushrooms sifted (1, coll. Schülke), same but leg. D.W. Wrase (14) (1, coll. Schülke); Yunnan, 100 km W Kunming, Diaolin Nat. Res., 22.V.-2.VI.1993, leg. E. Jendek \& O. Šauša (3, NHMW); Yunnan, Kunming, Western Hills, $25^{\circ} 04^{\prime} \mathrm{N}, 102^{\circ} 41^{\prime} \mathrm{E}, 1800$ m, 24.VII.1996, leg. A. Smetana, J. Farkač, P. Kabátek (C68), mixed secondary forest, sifting of still green leaves and underlying detritus under branches of freshly cut large shrub (22, coll. Smetana, 
NSMT, 3 ô, CNCI); Yunnan, N-, Nujiang Lisu Aut. Pr., Gongshan Co., Gaoligong Shan, above ranger station, $27^{\circ} 47.65^{\prime} \mathrm{N}, 98^{\circ} 35.41^{\prime} \mathrm{E}, 2000 \mathrm{~m}, 19 . \mathrm{VI} .2005$, leg. A. Smetana (C166), sifting of moss, leaf litter and other debris accumulated between large rocks in disturbed broadleaved forest ( 1 + , CNCI); Fujian prov., Wuyi Shan Nat. Res., Masu, 1100 m, 6. VI.2001, leg. P. Hlaváč \& J. Cooter (1, HNHM); Jiangxi prov., Wuyi Shan Nat. Res., Huangganshan, 1800-2050 m, 5.VI.2001, leg. P. Hlaváč \& J. Cooter (1, SDEI, 1, FMNH, 1, ZMUC, 1, ISNB, 1, AMNH, 1, NIBR, 1, NHMW, 1, CNCI, 1, MNHP, 1, MHNG, 1 , , HNHM, 1 $\lesssim, 1$

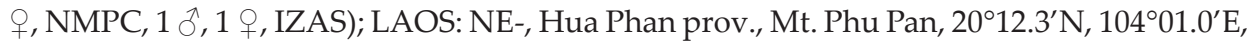
1500-1900 m, 17.V.-3.VI.2007, leg. C. Holzschuh (1 q, NHMW); Phongsaly prov., env. Phongsaly, $21^{\circ} 41.2^{\prime} \mathrm{N}, 102^{\circ} 06.6^{\prime} \mathrm{E}, 1500 \mathrm{~m}, 28 . V .-20 . V I .2003$, leg. C. Holzschuh (3 o, NHMW); THAILAND: Tak prov., Taksin Maharat National Park, 1000 m [16 $51^{\circ} 34^{\prime \prime} \mathrm{N}$, 9849'51"E], 9.II.1993, leg. P. Schwendinger (3, MHNG, 1, HNHM); Tak prov., road from Mac Sot to Umphang, mountains N Umphang, 1250 m, 10.II.1993, leg. P. Schwendinger, evergreen hill forest (1, MHNG); Chiang Mai prov., Doi Suthep, versant sud, 1450 m, 4. XI.1985, leg. D. Burckhardt \& I. Löbl (5), tamisage de débris végétaux dans un ravin assez humide (5, MHNG); Chiang Mai prov., Doi Suthep, versant nord, 1400 m, 5.XI.1985, leg. D. Burckhardt \& I. Löbl (11), ravin très humide, tamisage de feuilles mortes près d'un ruisseau (4, MHNG); Chiang Mai prov., Doi Inthanon, 1650 m, 7.XI.1985, leg. D. Burckhardt \& I. Löbl (16), ravin très humide, tamisage de débris végétaux et de champignons près d'un ruisselet (37, MHNG); Chiang Mai prov., Doi Inthanon, 2450 m, 9.XI.1985, leg. D. Burckhardt \& I. Löbl (19), tamisage de feuilles mortes à la lisière de la forêt, sur une pente raide (6, MHNG); Chiang Mai prov., Doi Inthanon, 1780 m, 3.III.1987, leg. P. Schwendinger (7,

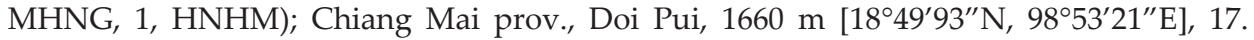
XI.1986, leg. P. Schwendinger (1, MHNG); Chiang Mai prov., Fang distr., Doi Angkhang, 10 km W Fang, 1500 m, 20.III.1987, leg. P. Schwendinger (3, MHNG); Chiang Mai prov., Fang distr., Doi Angkhang, 10 km W Fang, 1460 m, 21.V.1986, leg. P. Schwendinger (2, MHNG); Mae Hong Son [bordering Mae Taeng?] prov., Doi Chang, 20 km E Pai, $1930 \mathrm{~m}$ [19²1'17"N , 98 35'28"E], 4.VI.1986, leg. P. Schwendinger, tamisage (2, MHNG); Mae Hong Son prov., Doi Chang, 20 km E Pai, 1950 m [19²1'17”N, 98³5’43”E], 10.IV.1987, leg. P. Schwendinger (1, MHNG); Mae Hong Son prov., Ban Maeo Microwave, 1250 m, 15. XII.1990, leg. P. Schwendinger (2, MHNG); VIETNAM: N-, 6 km NW Sa Pa, 22²1'39"N, $103^{\circ} 47^{\prime} 19^{\prime \prime} \mathrm{E}, 1810 \mathrm{~m}, 7 . \mathrm{VIII} .2015$, leg. V. Assing (8), sifted from litter and roots under bushes and small trees at the margin of a pasture (1, coll. Assing); N-, $10 \mathrm{~km} \mathrm{NW} \mathrm{Sa} \mathrm{Pa,}$ $22^{\circ} 22^{\prime} 26^{\prime \prime} \mathrm{N}, 103^{\circ} 45^{\prime} 27^{\prime \prime} \mathrm{E}, 1850 \mathrm{~m}, 8$.VIII.2015, leg. V. Assing (9), sifted from roots and moist leaf litter in a steep stream valley with deciduous trees (5, coll. Assing); Ninh Binh, Cuc Phuong, 18.X.1986, leg. F. Mészáros, J. Oláh \& T. Vásárhelyi (80), singling (2, HNHM); N-,

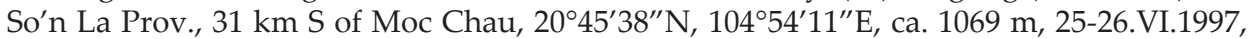
leg. C. L. Li (1 3 , coll. Schülke); Ha Tinh pr., Raò An, 13 km W Huang Son, rt. 8, 7 km S Nuòc Sôt village, $18^{\circ} 21^{\prime} \mathrm{N}, 1^{\circ} 5^{\circ} 5^{\prime} \mathrm{E}, 220$ m, 26.IV.1998, leg. L. Herman, litter near stream (1, AMNH), same but 300-350 m, 1.V.1998, litter near forest stream (1, AMNH).

Redescription - Measurements (in mm, $\mathrm{n}=10)$ : $\mathrm{HW}=0.76(0.69-0.81)$; $\mathrm{TW}=0.80(0.73-$ $0.84) ; \mathrm{PW}=1.06$ (0.99-1.19); $\mathrm{EW}=1.15$ (1.09-1.38); $\mathrm{AW}=1.19$ (1.09-1.35); $\mathrm{HL}=0.58$ (0.52$0.62) ; \mathrm{EL}=0.15(0.14-0.17) ; \mathrm{TL}=0.23(0.20-0.24) ; \mathrm{PL}=0.62$ (0.55-0.72); $\mathrm{SC}=0.67$ (0.62-0.84); $\mathrm{FB}=1.96(1.79-2.24) ; \mathrm{BL}=3.77$ (3.27-4.00). Habitus as in Fig. 101. Forebody moderately shining despite sculpture, abdomen very finely microsculptured and shallowly punctured, less lustrous. Body reddish dark brown but clypeal area lighter, reddish (abdomen occasionally also lighter); mouthparts and antennae medium brown. Head with anterior margin forming an arch more or less truncate in middle, an extremely thin rim, supraan- 


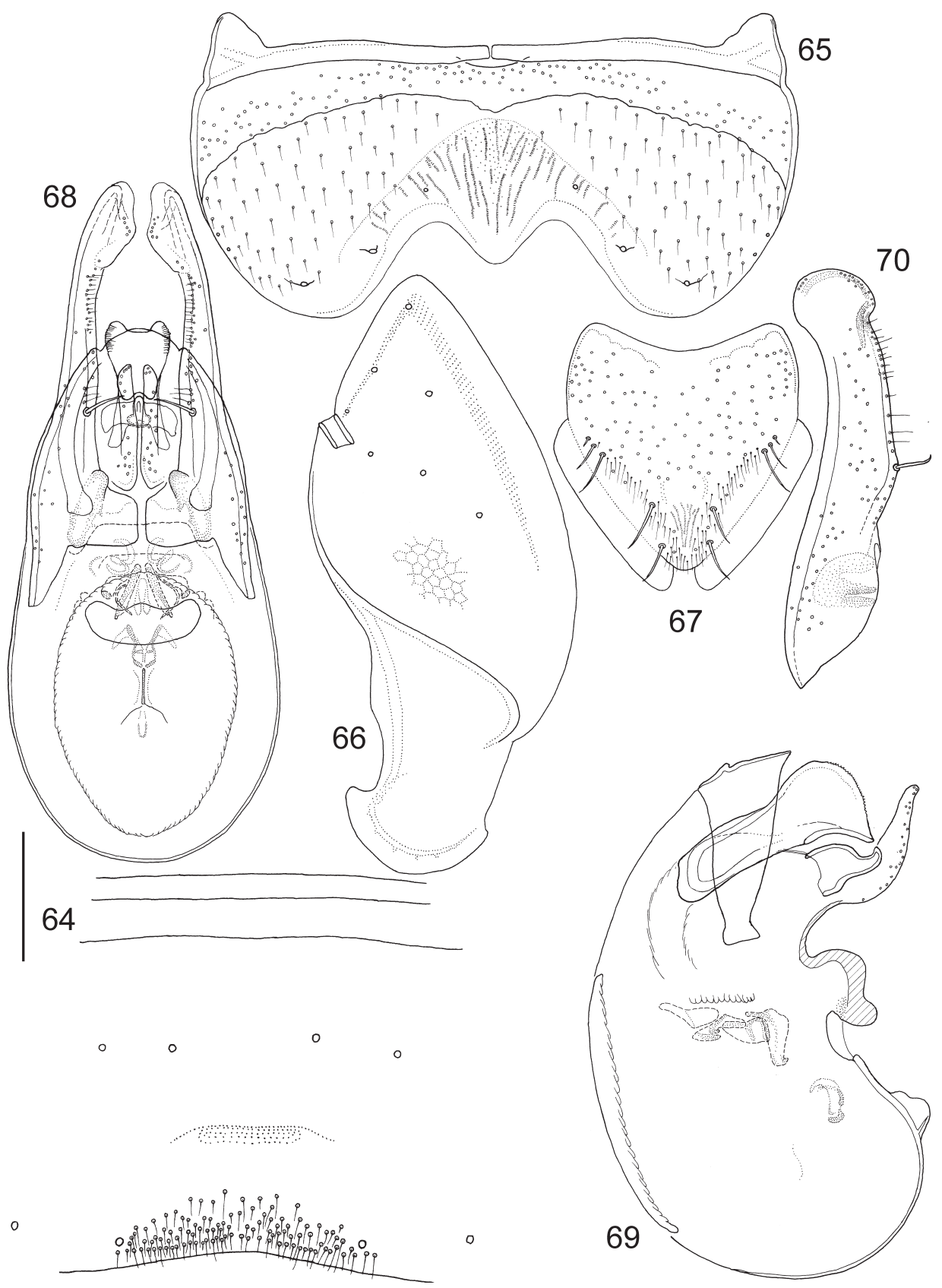

Figs 64-70. Anotylus excisicollis (Bernhauer, 1938), male. $64=$ median part of sternite VII, $65=$ sternite VIII, $66=$ tergite IX, $67=$ tergite $X, 68=$ aedeagus, frontal view, $69=$ median lobe, lateral view, 70 = paramere, lateral view. Scales: $0.10 \mathrm{~mm}$ for Figs $68-70,0.125 \mathrm{~mm}$ for Fig. 64, $0.15 \mathrm{~mm}$ for Figs 65-67 
tennal prominences as moderately elevated oblique ridges, disc slightly impressed near eyes, middle of vertex slightly elevated. Epistomal suture forming subcircular impression (less punctured inside), upper surface without tactile setae, eyes medium large, moderately convex; in dorsal view temples (1.5× length of eye) alatiform, laterally angled or more rounded and posteriorly constricted to form distinct neck, however, dorsal side not sep-
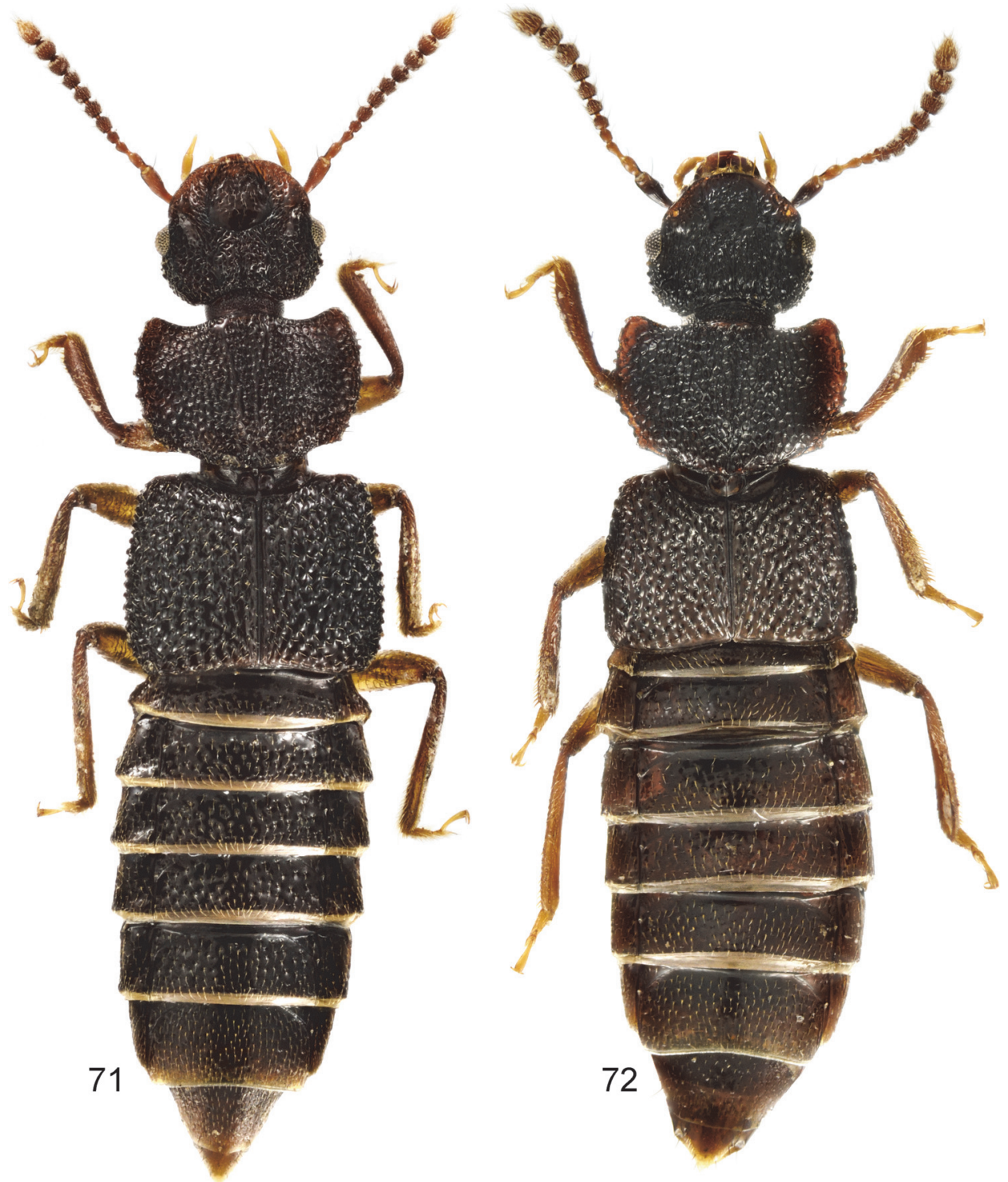

Figs 71-72. Habitus of Anotylus species. $71=$ A. apicipennis (Fauvel, 1895), $72=$ A. ganapati sp. n. 


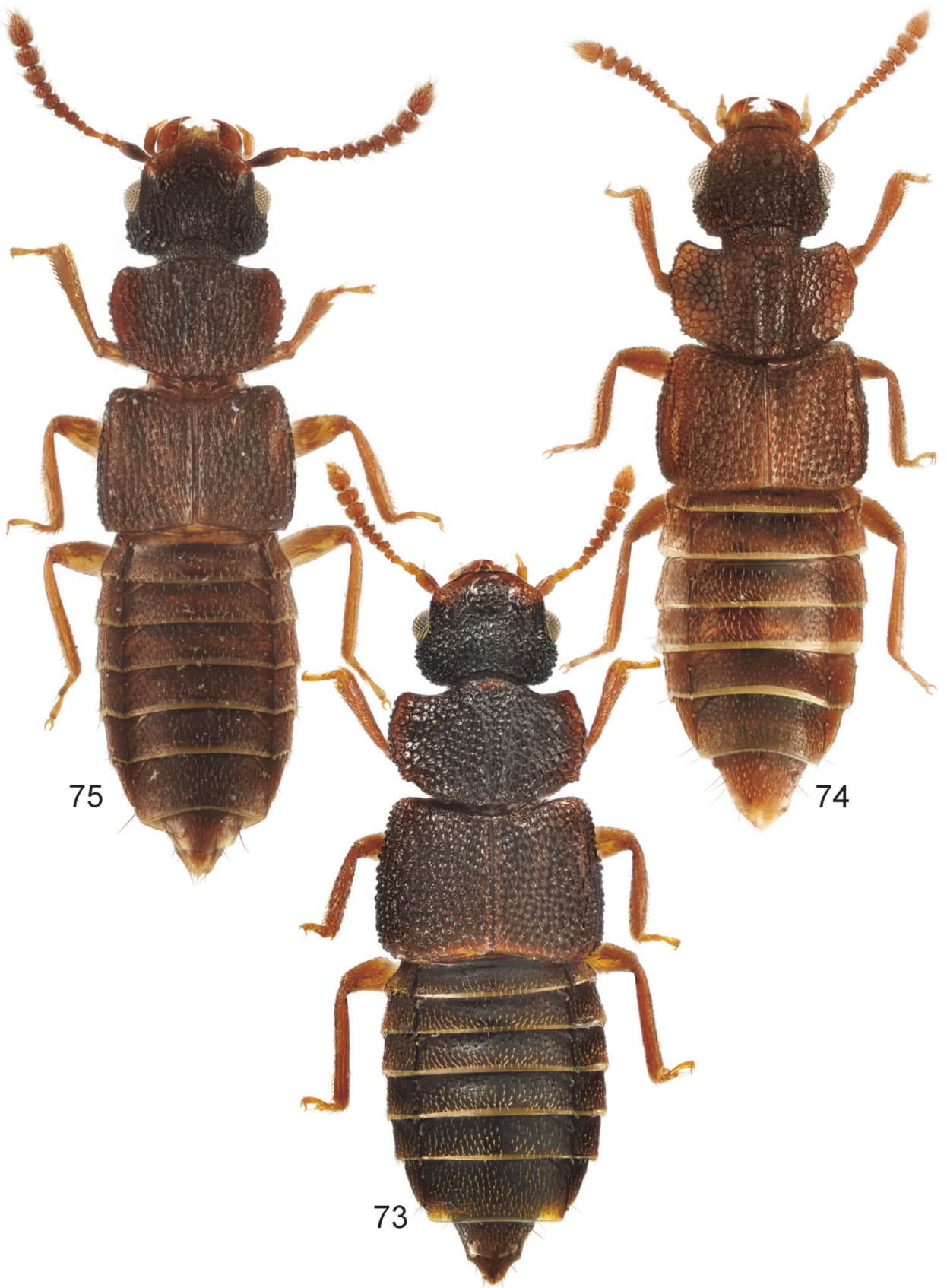

Figs 73-75. Habitus of Anotylus species. $73=$ A. schawalleri sp. n., $74=$ A. borneensis (Cameron, 1928), $75=$ A. gunung sp. n. 
arated by occipital furrow, rather by dorsum of neck bearing microsculpture instead of punctation. Antennomere 1 flattened club-shaped and finely microsculptured, segment 2 oval, segment 3 club-shaped, segment 4 almost round, segments 5-11 with basal dishes, segments 6-10 transverse (almost $2 \times$ broader than long). Head and pronotum with mostly lacunose sculpture. Pronotum with lateral portion explanate and slightly up-turned, edge alatiform with sparse short setae; lateral border uneven and with a number of small, irregularly distributed denticles, posterior margin incised before corners; disc medially with two parallel and posteriorly almost confluent longitudinal ridges, posteriorly impressed alongside them, laterally impressed around middle of sides. Elytra with membranous posterior margin extending from sutural corner to 5/6 of hind margin, a small incision before outer corner. Elytral epipleural ridge present with up-turned lateral alatiform portions,

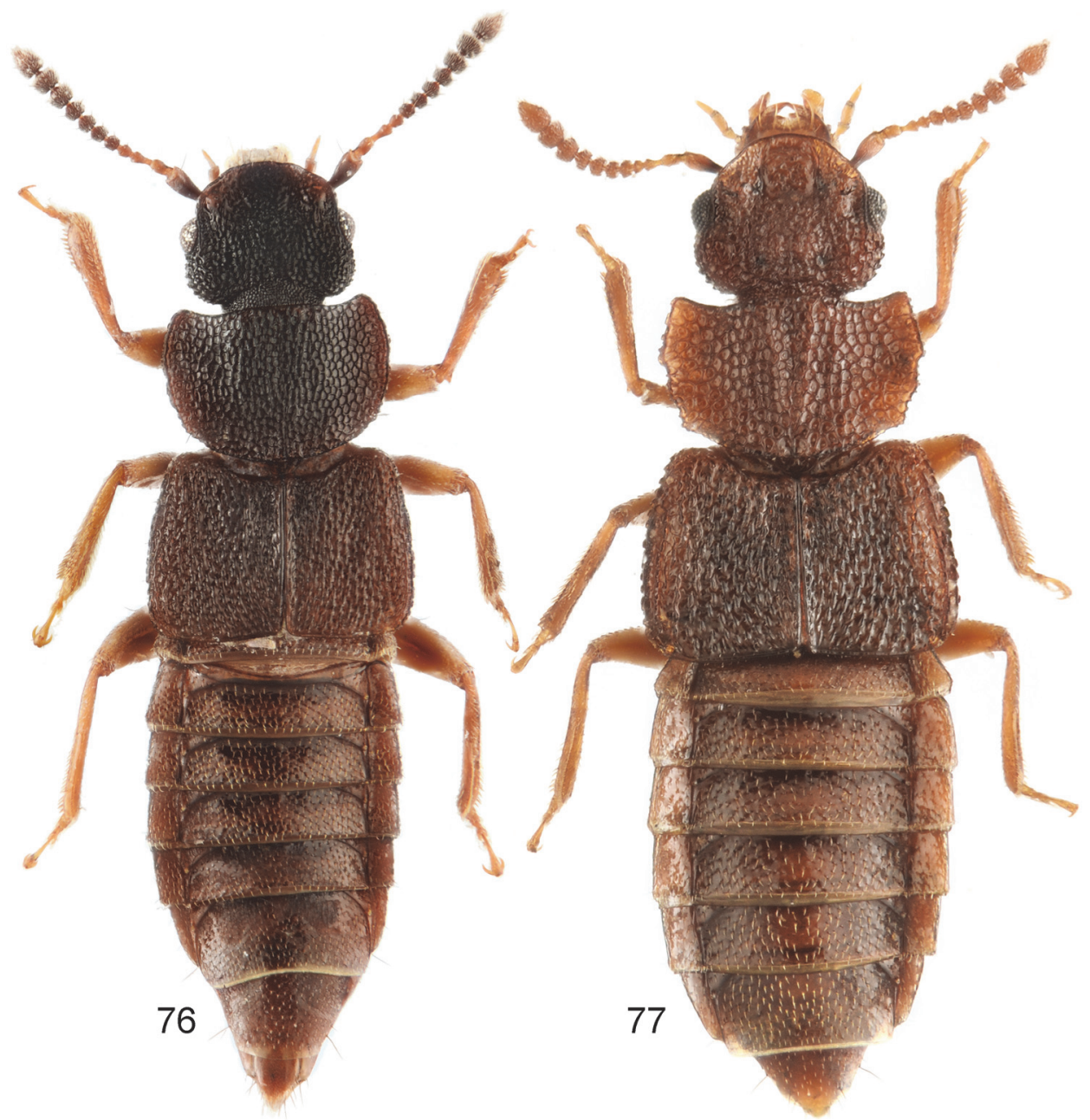

Figs 76-77. Habitus of Anotylus species. $76=$ A. coonoor sp. n., $77=$ A. linaxi sp. $\mathrm{n}$. 
disc longitudinally impressed alongside; shoulders well developed, surface foveolate, in between punctures torulose. Legs short, pro- and mesotibia with several spinulose rows, metatibia with longitudinal ctenidium of spinules in distal half. Abdomen with sides gently arcuate, second segment with paratergites strongly broadening posteriorly (abdomen appearing constricted at base), those of segments III-VII thin, mesal paratergites broad; posterior edge of tergite VII with thin palisade fringe, median part of sternite VII as in Fig. 64, sternite VIII (Fig. 65), tergites IX (Fig. 66), X (Fig. 67) and aedeagus as in Figs 68-70.

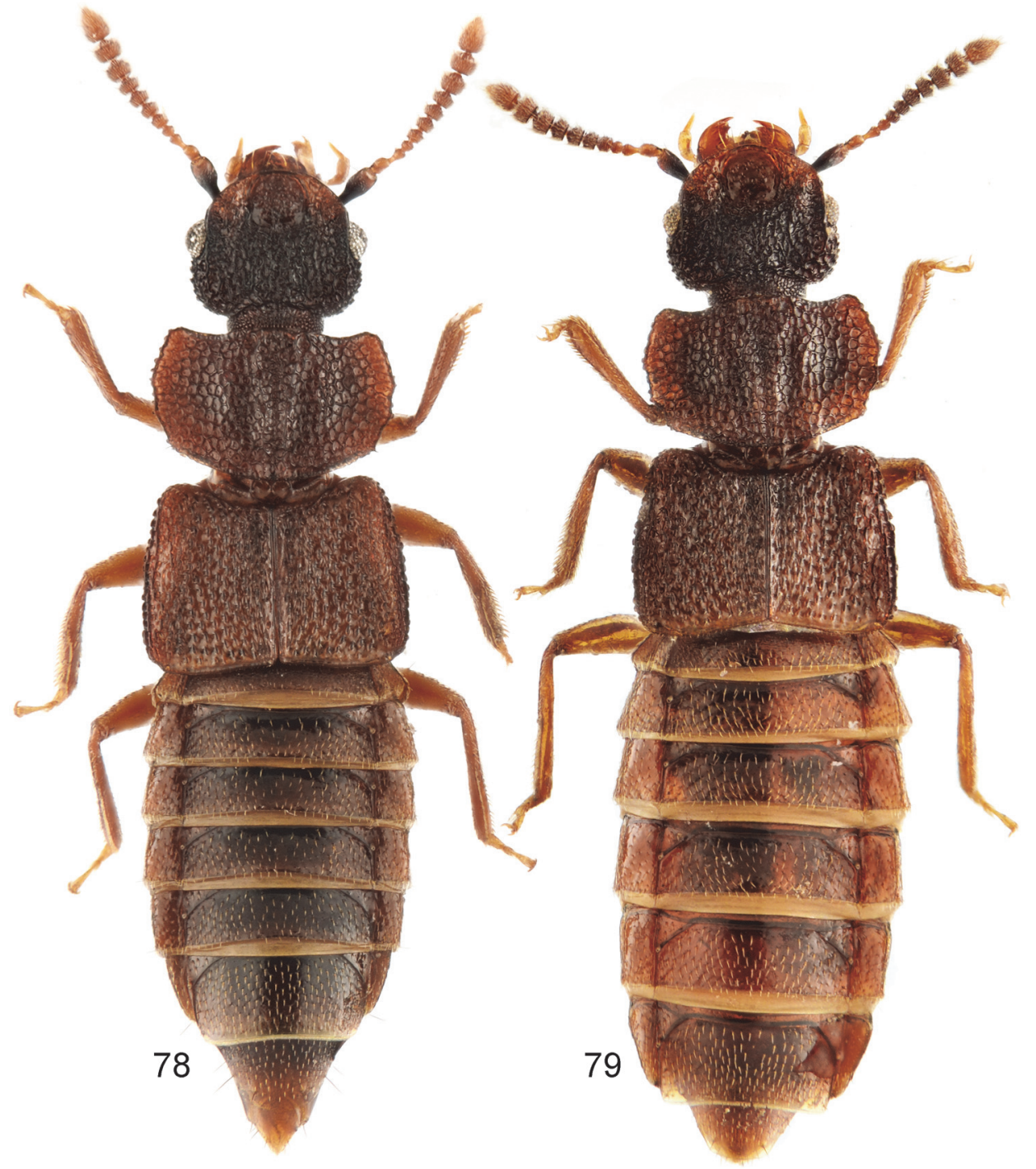

Figs 78-79. Habitus of Anotylus species. $78=$ A. chinensis (Bernhauer, 1938), $79=$ A. reitteri (Bernhauer, 1934) 
Distribution - The currently known distribution is the Indian Himalaya, Nepal, southern China, Myanmar, N Thailand, Laos and Vietnam.

Remarks - The type material is a single female and it is, of course, impossible to test it against differentiation of species based on male characters. For this reason the identity may require reinvestigation in the future.

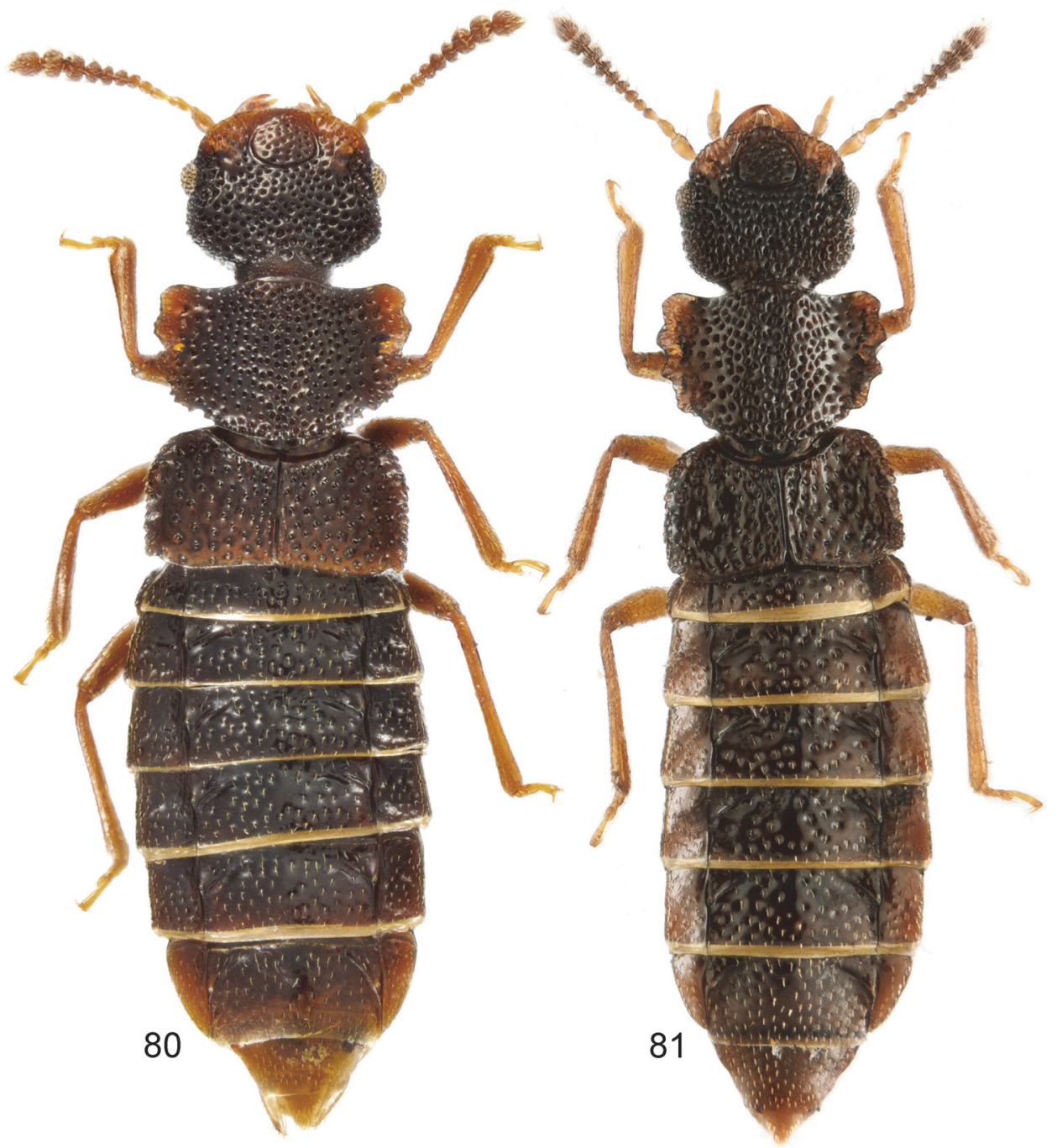

Figs 80-81. Habitus of Anotylus species. $80=$ A. ceylonicus (Hammond, 1975), $81=$ A. montanus (Hammond, 1975) 


\section{Anotylus franzi (Coiffait, 1982) \\ (Figs 86, 104-110)}

Oxytelopsis franzi Coiffait, 1982: 162; Coiffait 1984: 150; Herman 2001: 1413.

Examined type material - Oxytelopsis franzi Coiffait - Neotype ( $\hat{\sigma}$, here designated): "NEPAL: (prov. Bagmati); below Thare Pati; 3300 m, 10.V.[19]81; [leg. I.] Löbl \& [A.] Smetana; [on the backside:] 18b [tamisage de mousses, de fougères et de feuilles mortes d'érables sur une pente raide près d'un ruisseau] \Neotypus; Oxytelopsis; franzi Coiffait; des. Makranczy, $2015 \backslash$ Anotylus; franzi (Coiffait); det. Makranczy, 2015" (NHMW).

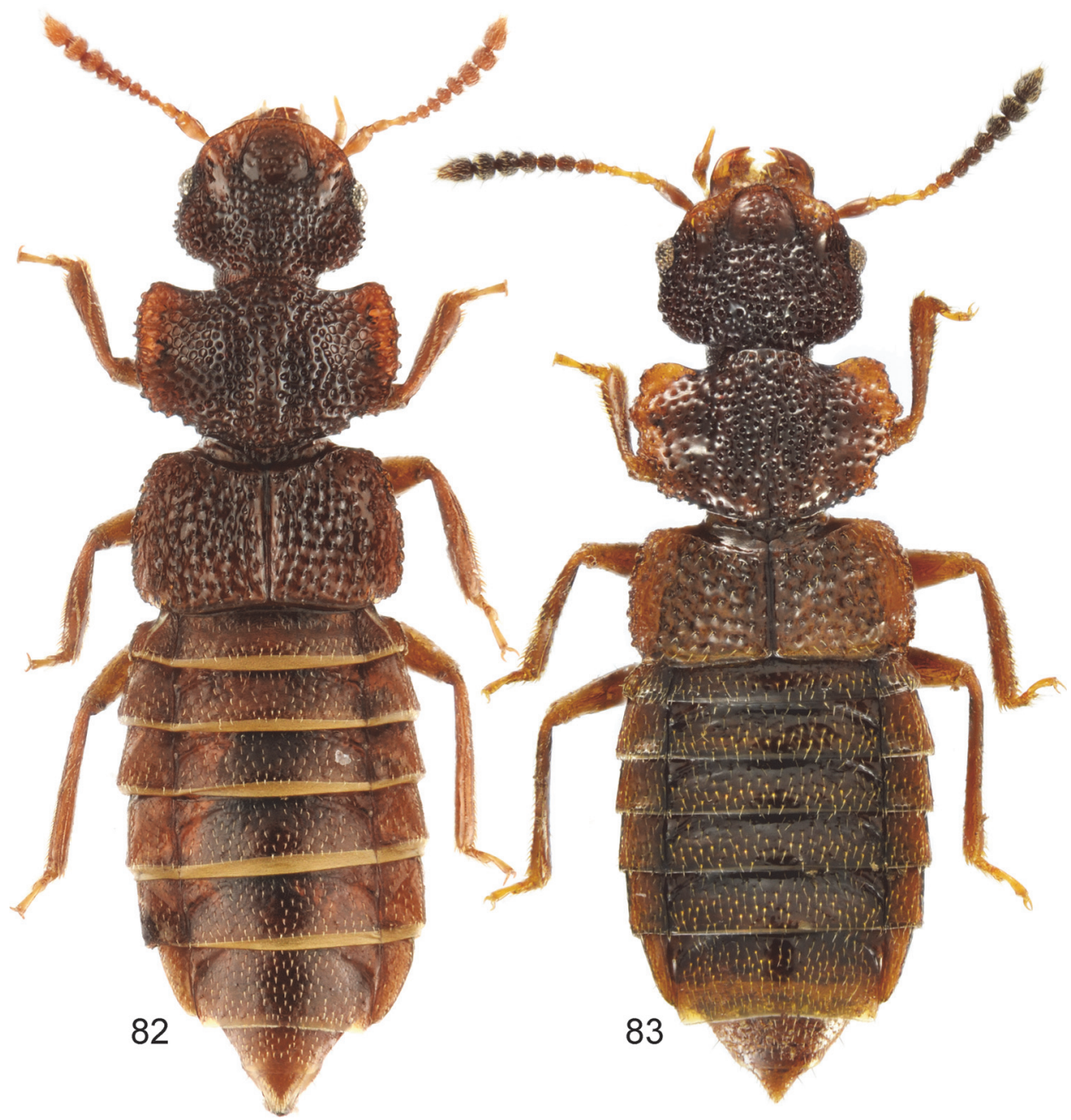

Figs 82-83. Habitus of Anotylus species. $82=$ A. hartmanni sp. n., $83=$ A. schillhammeri sp. $\mathrm{n}$. 
Other material - NEPAL: prov. Bagmati, Burlang Bhanjyang, 2600 m, 5.IV.1981, leg. I. Löbl \& A. Smetana $(7 \mathrm{~b})$, tamisage de mousses et de feuilles mortes près d'une source (2, MHNG, 1, ISNB); prov. Bagmati, au-dessus de Gul Bhanjyang, 2600 m, 6.IV.1981, leg. I. Löbl \& A. Smetana (9), vieille chênaie sur le versant nord, pente raide, tamisage de feuilles mortes, mousses et bois pourri, au pied des arbres (13, MHNG); prov. Bagmati, Malemchi, 2900 m, 14.V.1981, leg. I. Löbl \& A. Smetana (23), tamisage de branches pourries, feuilles mortes, herbe et mousses au pied d'une muraille près du village (1, MHNG); prov. Bagma-

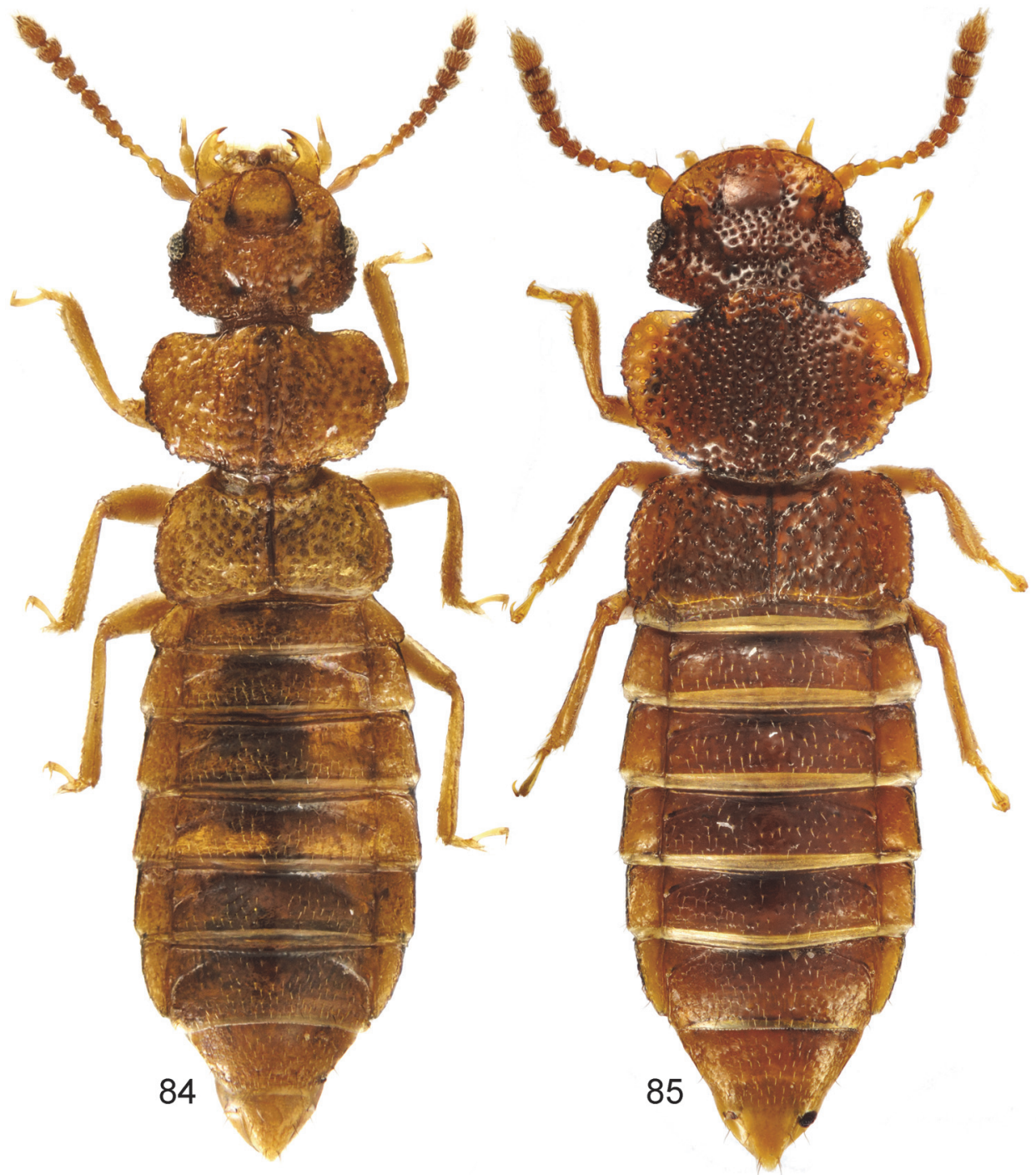

Figs 84-85. Habitus of Anotylus species. $84=$ A. schuelkei sp. n., $85=$ A. bengalensis (Biswas et Sengupta, 1980) 
ti, Malemchi, 2800 m, 14.V.1981, leg. I. Löbl \& A. Smetana (24), tamisage de branches pourries, feuilles mortes, herbe et mousses au pied d'une muraille près du village $(9, \mathrm{MHNG}$, 1, CNCI, 1, NIBR, 1, ZMHB, 1, HNHM); prov. Bagmati, Malemchi, 2900 m, 18.V.1981, leg. I. Löbl \& A. Smetana (30), mousses sur les tsuga, tamisage; tamisage de bois pourri dans un chêne creux $(9, \mathrm{MHNG})$; prov. Bagmati, Tarke Ghyang [ $\left.28^{\circ} 00.0^{\prime} \mathrm{N}, 85^{\circ} 33.5^{\prime} \mathrm{E}\right], 2650 \mathrm{~m}$, 19.IV.1981, leg. I. Löbl \& A. Smetana (32a), tamisage de feuilles mortes et de mousses dans un ravin près d'un ruisseau (4, MHNG, 1 , NKME, 1, SDEI); prov. Bagmati, above Shermathang, 2900 m, 26.IV.1981, leg. I. Löbl \& A. Smetana (47c), tamisage de feuilles mortes et fougères entre les roches dans une forêt de chênes-rhododendrons (4, MHNG, 1, BMNH, 1 , MNHP, 1, ZMUC, 1, FMNH, 1, NMPC, 1, SMNS, 1, AMNH, 1 §̊, 1, HNHM).

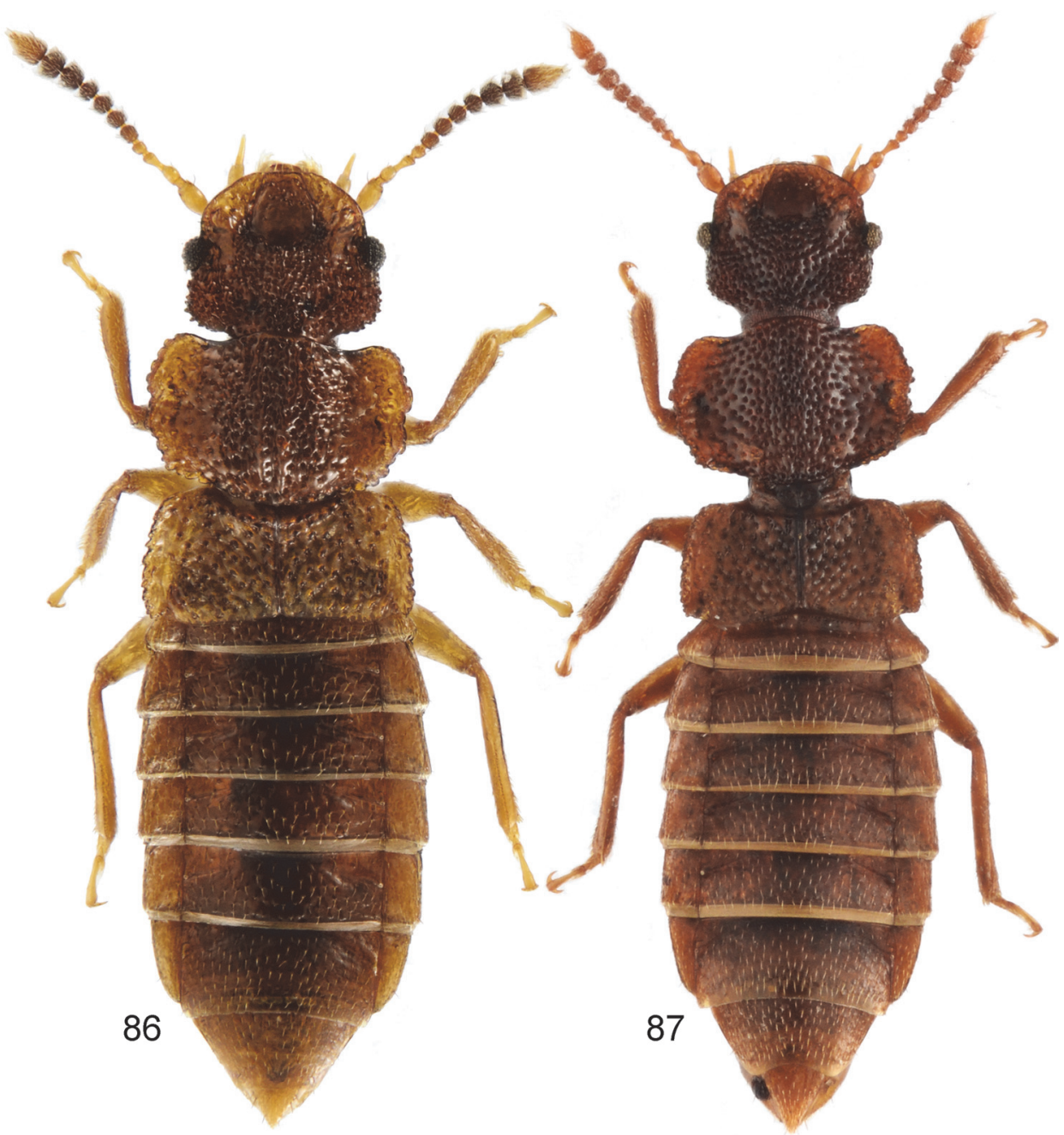

Figs 86-87. Habitus of Anotylus species. $86=$ A. franzi (Coiffait, 1982), $87=$ A. jaljulensis

(Coiffait, 1984) 
Redescription - Measurements (in mm, $\mathrm{n}=10)$ : $\mathrm{HW}=0.60(0.58-0.61) ; \mathrm{TW}=0.58$ (0.56-0.60); $\mathrm{PW}=0.79(0.75-0.84) ; \mathrm{EW}=0.81(0.78-0.84) ; \mathrm{AW}=0.86(0.82-0.91) ; \mathrm{HL}=0.63$ (0.46-0.49); $\mathrm{EL}=0.105(0.10-0.11) ; \mathrm{TL}=0.19(0.18-0.20) ; \mathrm{PL}=0.52(0.50-0.54) ; \mathrm{SC}=0.38$ $(0.36-0.39) ; \mathrm{FB}=1.42$ (1.38-1.45); $\mathrm{BL}=2.90$ (2.75-3.06). Habitus as in Fig. 86. Forebody moderately shining despite sculpture, abdomen with coriaceous microsculpture, moderately finely and shallowly punctured, less lustrous. Body reddish medium to dark brown except anterior margin of head, lateral margin of pronotum, antennomeres 1-4 and mouthparts medium brown, apex of last antennomere occasionally also lighter. Head with anterior margin truncate in middle, oblique to supraantennal tubercles (as moderately elevated oblique ridges) where gently angled again, possessing slight rim (obsolete at front), disc slightly impressed near eyes. Epistomal suture forming subcircular impression (almost unpunctured inside), upper surface without tactile setae, eyes small and extremely bulging

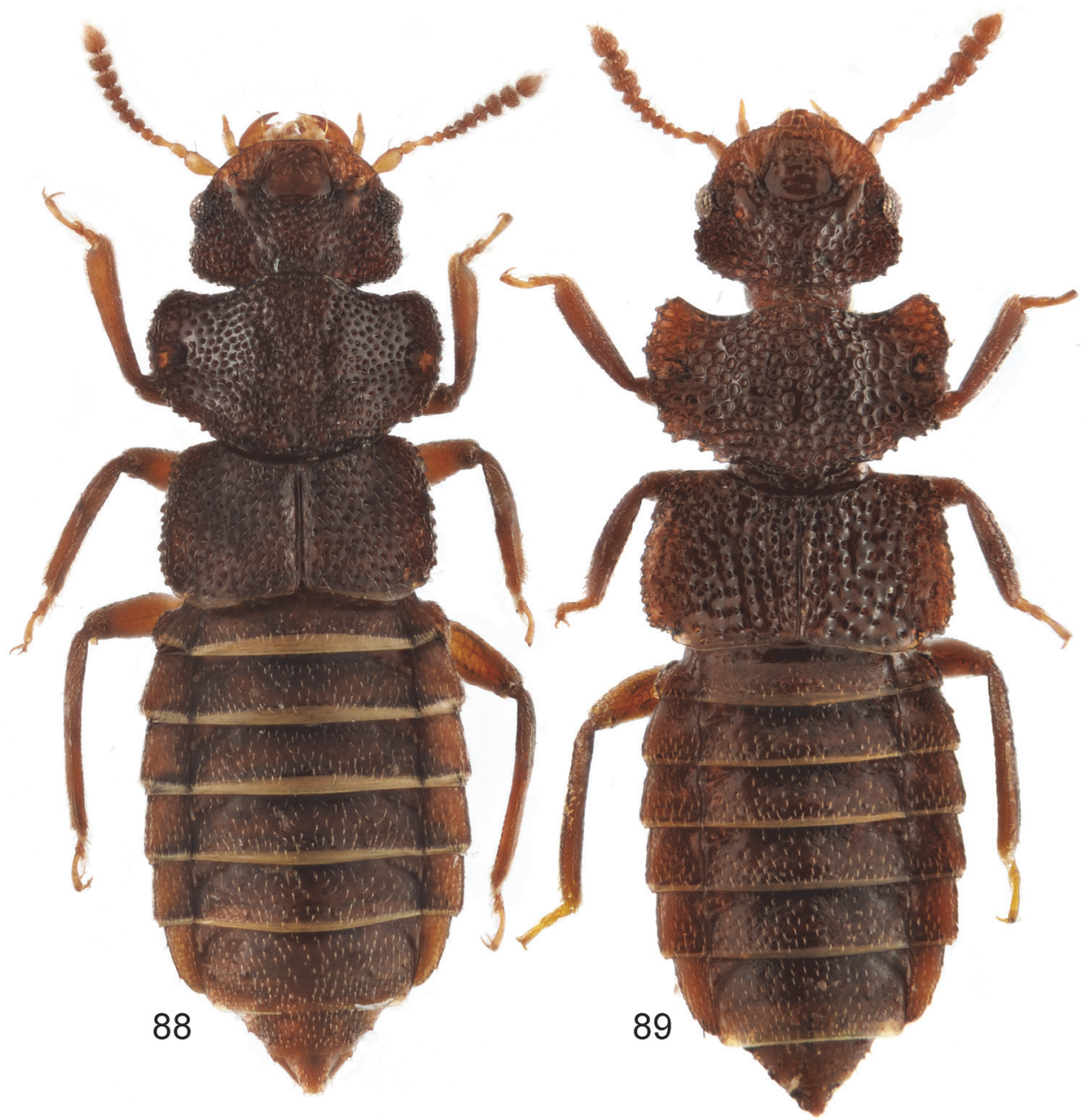

Figs 88-89. Habitus of Anotylus species. $88=$ A. jambi sp. n., $89=$ A. ijen sp. n. 
(like half sphere); in dorsal view temples (1.8× length of eye) alatiform, laterally slightly broadening and angled, posteriorly constricted to form distinct neck, however, dorsal side not separated by occipital furrow, rather by dorsum of neck bearing microsculpture instead of punctation. Antennomere 1 flattened club-shaped and smooth, segment 2 basally broad ovoid, segment 3 club-shaped, segment 4 small and isodiametrical, segments 5-11 with basal dishes, articles 6-10 slightly transverse (about $1.3 \times$ broader than long). Head and pronotum with mostly lacunose sculpture. Pronotum with lateral portion explanate and slightly up-turned, edge with a few short setae, lateral border almost evenly arched, without posterior corners, with a number of small, irregularly distributed tubercles; disc medially rather convex, with two faint, parallel longitudinal ridges, laterally impressed around middle of sides. Elytra with very thin membranous posterior margin extending

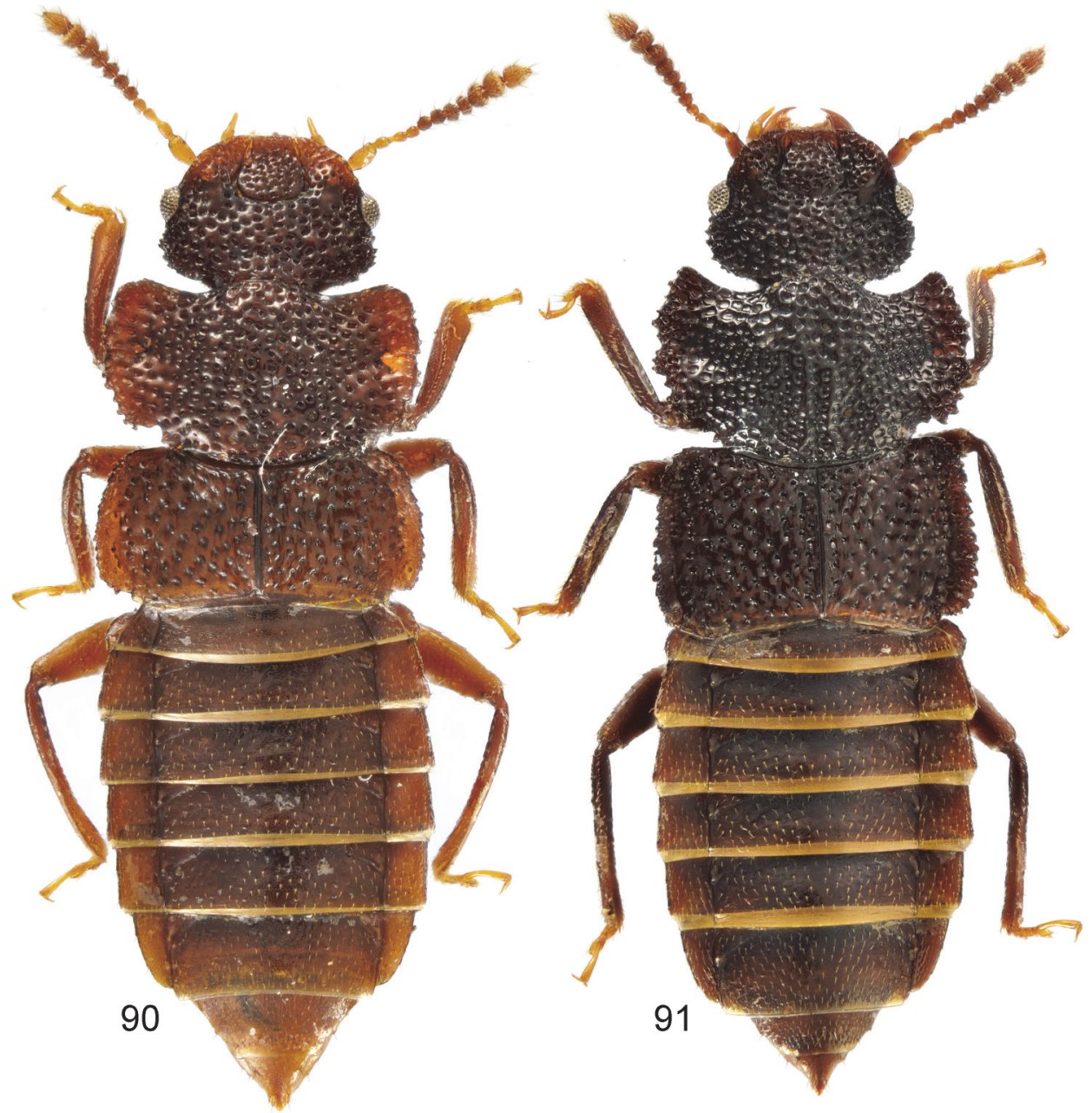

Figs 90-91. Habitus of Anotylus species. $90=$ A. lucidulus (Cameron, 1936), $91=$ A. riedeli sp. $\mathrm{n}$. 
from sutural corner to lateral 3/4 of hind margin. Elytral epipleural ridge present with upturned lateral alatiform portions, disc depressed; shoulders moderately developed, surface foveolate, in between punctures torulose. Legs short, pro- and mesotibia with several spinulose rows, metatibia with longitudinal ctenidium of spinules in distal half in distal half. Abdomen with sides weakly arcuate, second segment with paratergites strongly broadening posteriorly (abdomen appearing constricted at base), those of segments III-VII thin, mesal paratergites broad. Posterior edge of tergite VII with thin palisade fringe, median part of sternite VII as in Fig. 104, sternite VIII (Fig. 105), tergites IX (Fig. 106), X (Fig. 107) and aedeagus as in Figs 108-110.

Distribution - Only known from a rather narrow area around the original type locality in central Nepal.

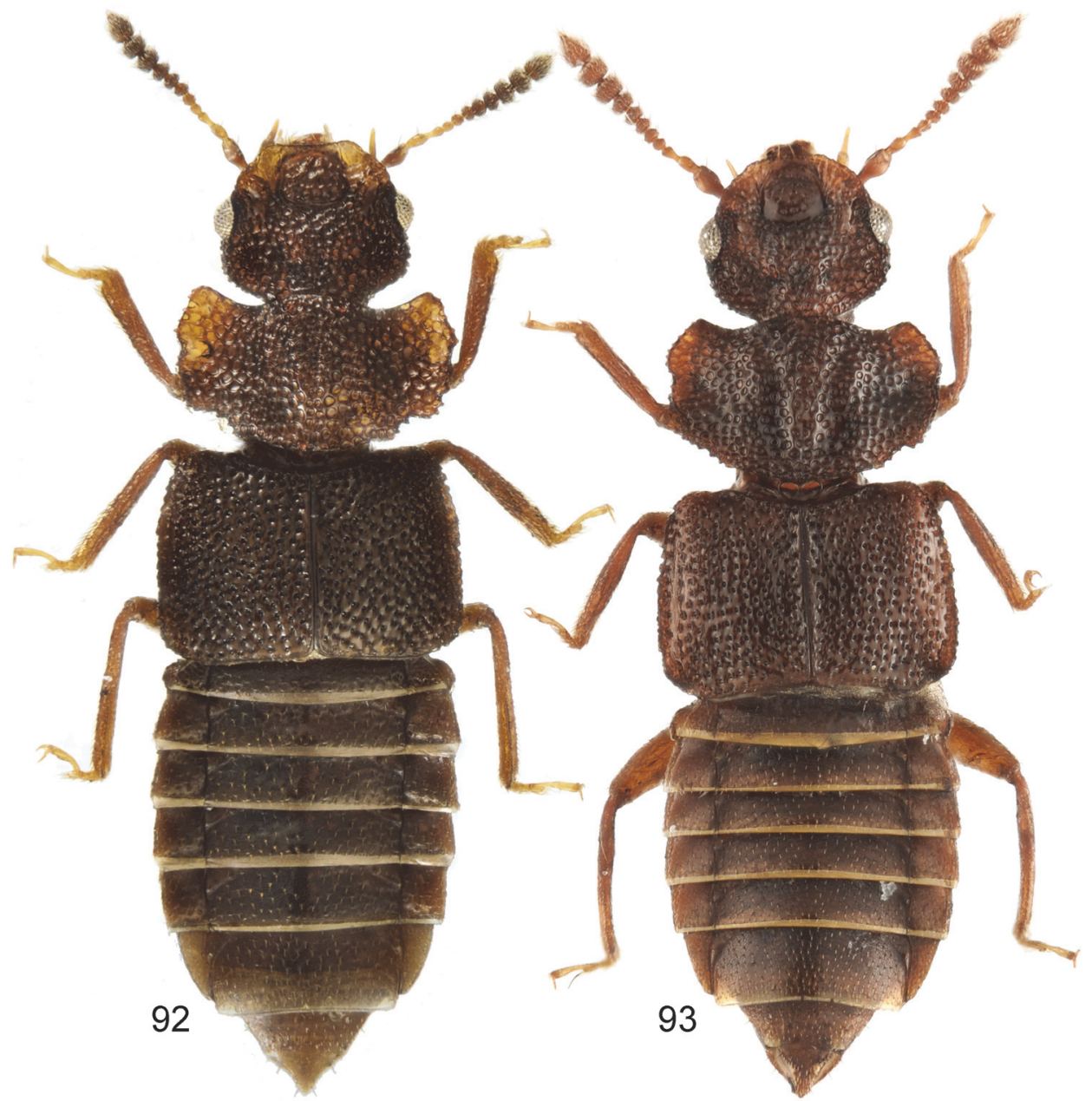

Figs 92-93. Habitus of Anotylus species. $92=$ A. andrewesi (Cameron, 1930), $93=$ A. topali sp. $\mathrm{n}$. 
Remarks - The original material of Oxytelopsis franzi is missing. Neither the holotype from "Népal (central), zw.[ischen] Mulk[h]arka und Tare Pati [Thare Pati, $\left.28^{\circ} 00^{\prime} 16^{\prime \prime} \mathrm{N}, 8^{\circ} 29^{\prime} 40^{\prime \prime} \mathrm{E}\right]^{\prime \prime}$ in H. Franz's collection (NHMW), nor the supposedly female paratype in the collection of $\mathrm{H}$. Coiffait (MNHP) are found in the depositories indicated in the description. The list of the author's type specimens (Coiffart 1986) does not even include Oxytelopsis franzi. Therefore a neotype must be designated to clarify the taxonomic status of the taxon - luckily, a suitable specimen exists from almost exactly the original

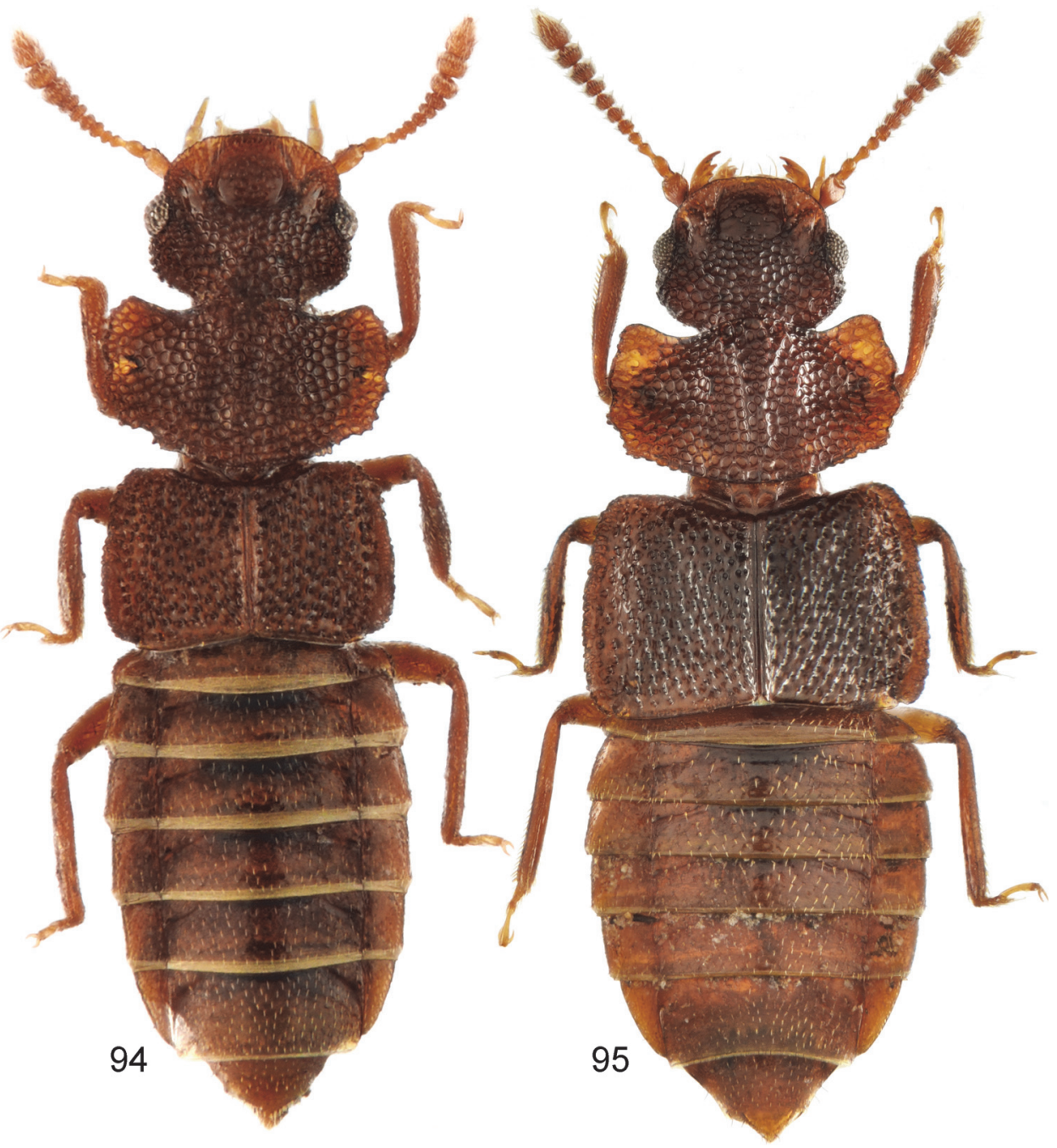

Figs 94-95. Habitus of Anotylus species. 94 = A. pseudopsinus (Fauvel, 1895), $95=$ A. malaisei (Scheerpeltz, 1965) 
type locality and therefore there is no doubt about the correct interpretation of the name.

\section{Anotylus ganapati sp. $\mathrm{n}$. \\ (Figs 72, 111-117, 266)}

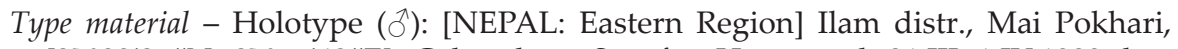
$2100 \mathrm{~m}\left[27^{\circ} 00^{\prime} 25^{\prime \prime} \mathrm{N}, 8^{\circ} 55^{\prime} 48^{\prime \prime} \mathrm{E}\right]$, Gebüsch an Seeufer, Hauswand, 31.III.-1.IV.1980, leg. J. Martens \& A. Ausobsky (117), Wald, Gesiebe (SMNS); Paratypes (90): Ilam distr., Mai

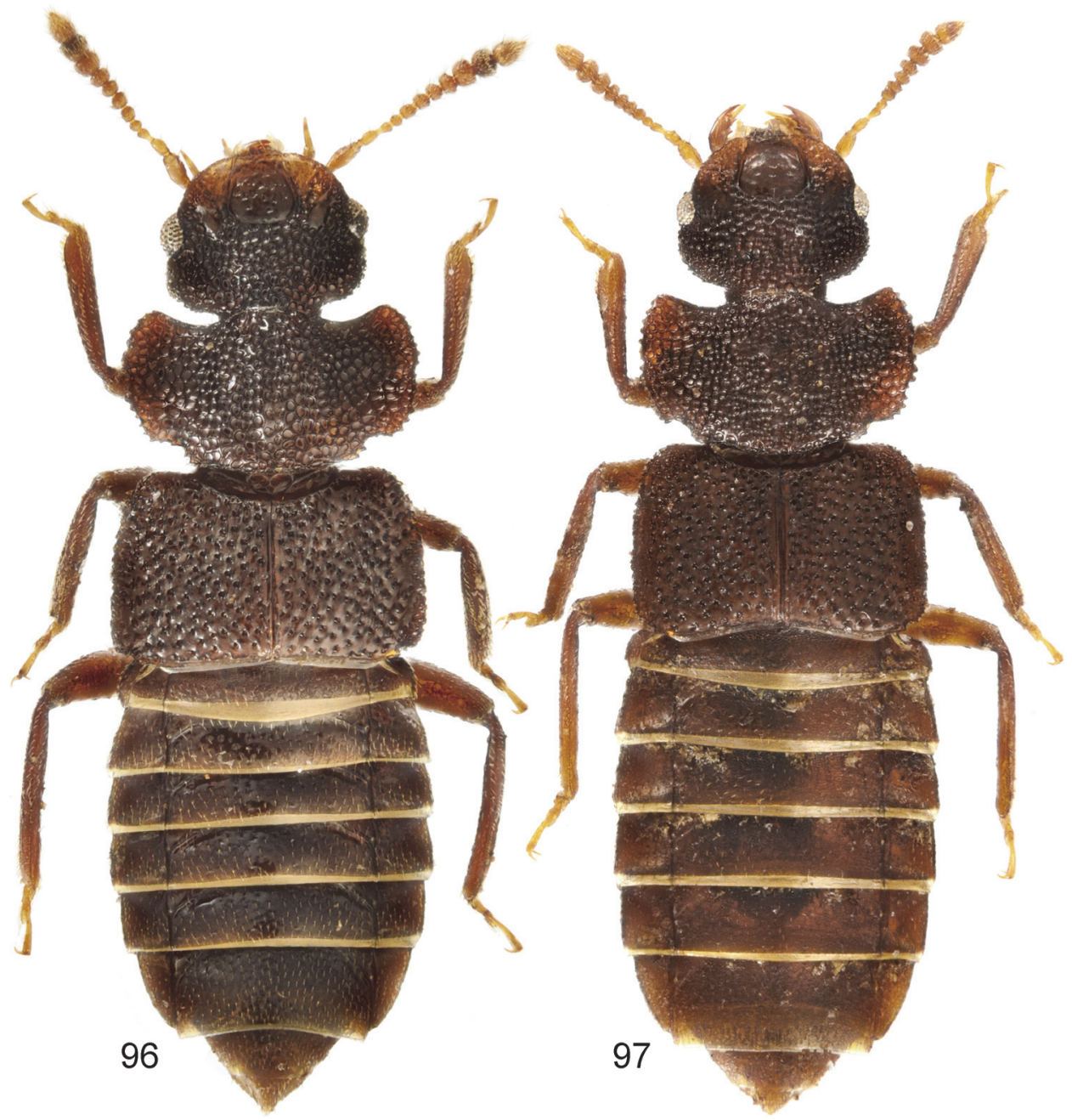

Figs 96-97. Habitus of Anotylus species. $96=$ A. rurukan sp. n., $97=$ A. tanator sp. $\mathrm{n}$. 
Pokhari, 2150-2250 m, 23-25.VIII.1983, leg. J. Martens \& B. Daams (254a), Berlese (1, SMNS); (Sankhuwasawa distr.) Kosi, forêt NE Kuwapani, ravin, 2350 m, 5.IV.1984, leg. I. Löbl \& A. Smetana (5), tamisage de feuilles mortes et humus à proximité d'une source (1, MHNG); (Sankhuwasawa distr.) Kosi, forêt NE Kuwapani, ravin, 2250 m, 6.IV.1984, leg. I. Löbl \& A. Smetana (6), tamisage de feuilles mortes le long d'un tronc abattu (3, MHNG); Kosi, Induwa Khola valley, 2000 m, 16.IV.1984, leg. I. Löbl \& A. Smetana (24a), tamisage mousses et feuilles mortes sur un terrain marécageux (1, MHNG, 1 ग , HNHM); Kosi, Induwa Khola valley, 2100 m, 17.IV.1984, leg. I. Löbl \& A. Smetana (27), tamisage mousses

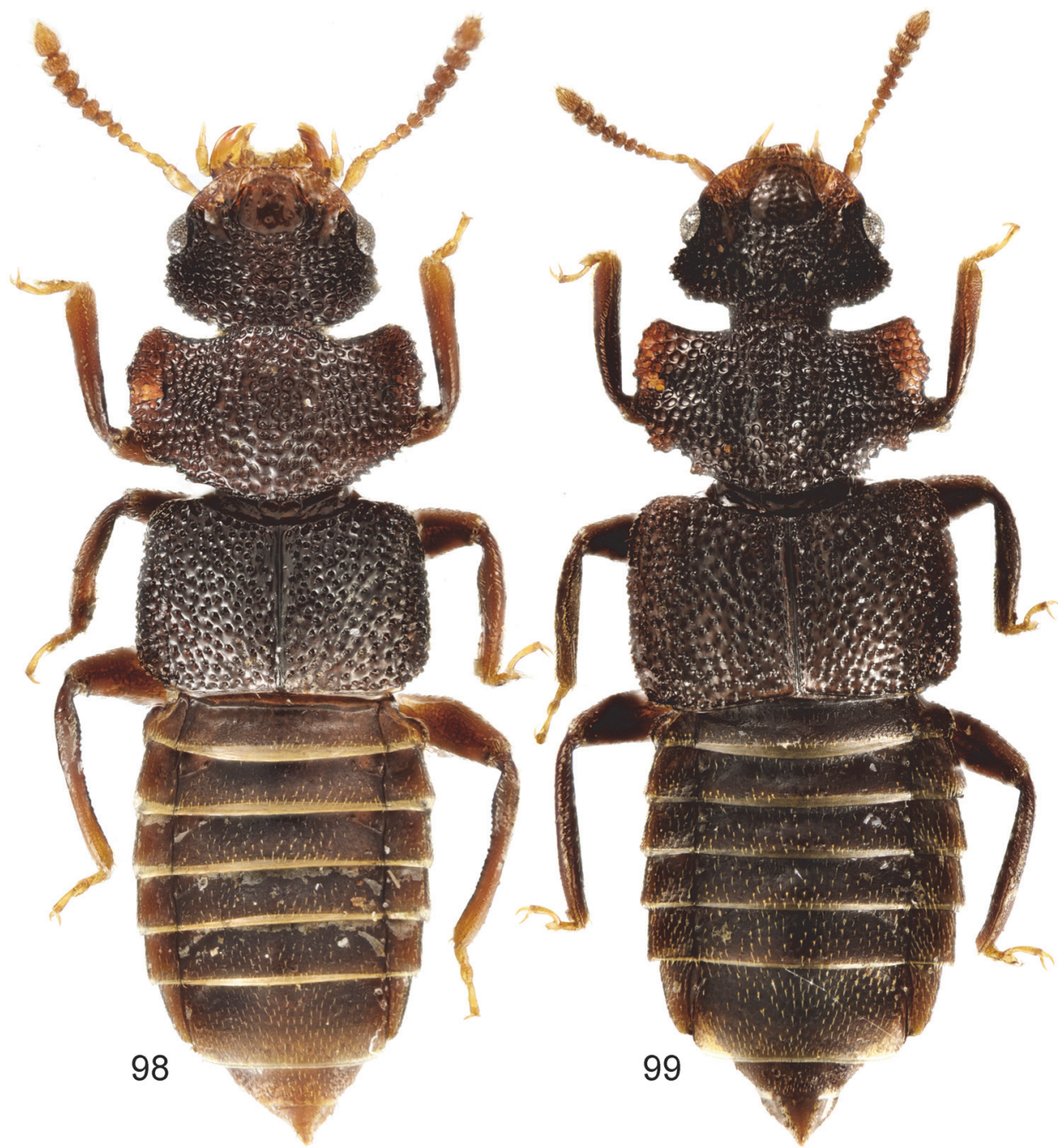

Figs 98-99. Habitus of Anotylus species. $98=$ A. kabasi sp. n., $99=$ A. nigricans (Cameron, 1933) 
et feuilles mortes au pied d'une falaise $(1 \hat{\delta}, 2$ + , MHNG, $1 \hat{\delta}, \mathrm{BMNH}, 1 \hat{\sigma}, \mathrm{CNCI}, 1 \hat{\delta}$, ZMHB); Kosi, Induwa Khola valley, 2000-2600 m, 16-18.IV.1984, leg. I. Löbl \& A. Smetana (30a), sous les pierres (2, MHNG); Kosi, forêt NE Kuwapani, 2250 m, 24.IV.1984, leg. I. Löbl \& A. Smetana (37), tamisage de branches pourries et mousses au-dessus d'un arbre couché (4, MHNG); Khandbari distr., Forest above Ahale, 2400 m, 25.III.1982, leg. A. \& Z. Smetana, original Quercus and Lauraceae forest, sifting of layers of moist and mouldy leaf litter, moss and detritus on and around fallen, rotting big trees (1, MHNG); Manaslu Mts., Dudh Pokhari Lekh, upper Dordi Khola Valley, 2300-2600 m, 15-17.IV.2003, leg. J. Schmidt (1, NKME); Manaslu Mts., Dudh Pokhari Lekh below Helam Pokhari, 2000 m, 22.IV.2003,

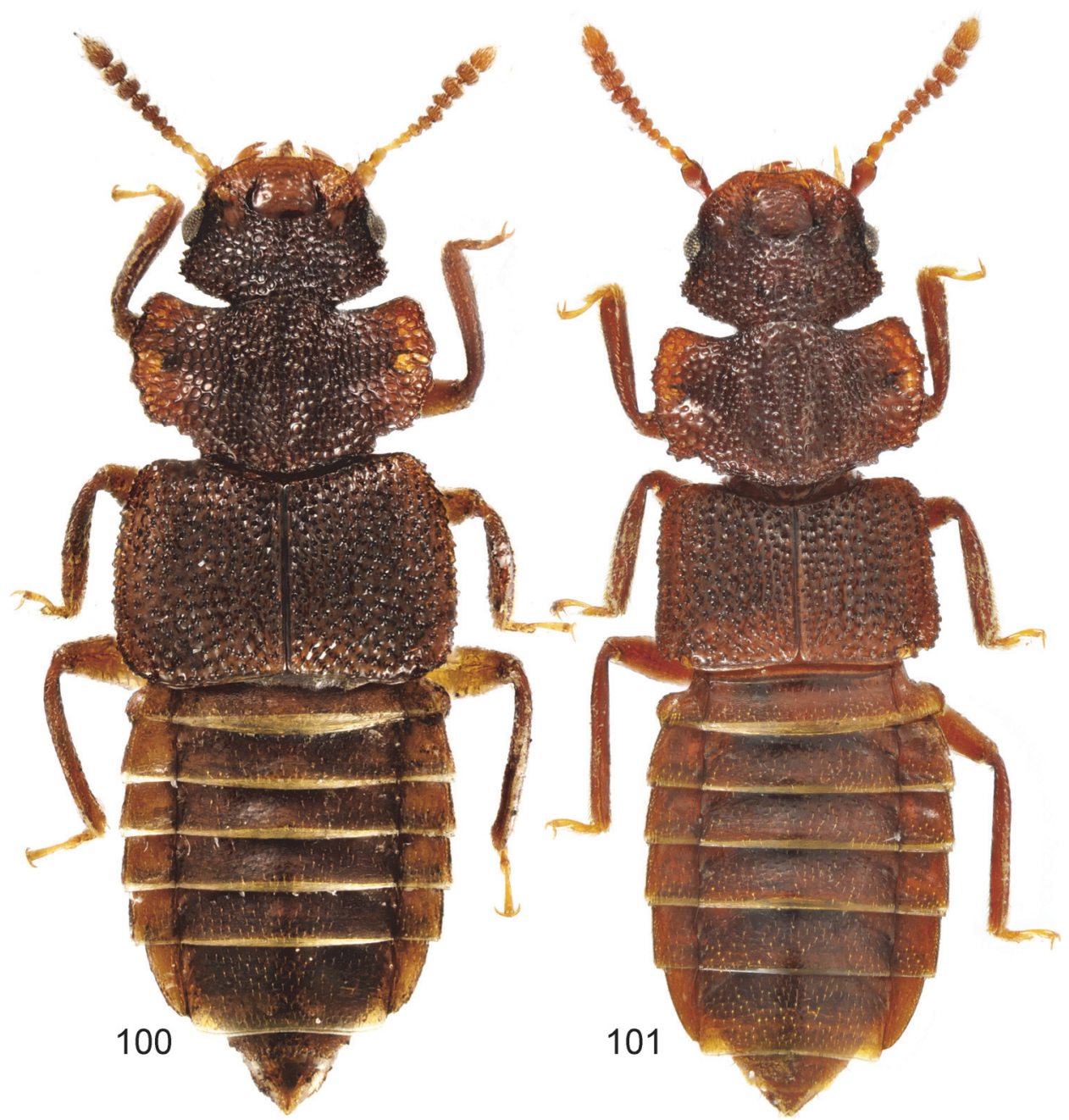

Figs 100-101. Habitus of Anotylus species. $100=$ A. cimicoides (Fauvel, 1895), $101=$ A. excisicollis (Bernhauer, 1938) 
leg. J. Schmidt, sifting forest litter (12, NKME, $1 \hat{\jmath}, \mathrm{NMPC}, 1 \hat{\jmath}, \mathrm{AMNH}, 1 \hat{\jmath}, \mathrm{ISNB}, 1 \hat{\jmath}$, NIBR, 1 $\delta$, ZMUC, 1 $\delta$, FMNH, 1 $\delta$, HNHM); Manaslu Mts., E slope of Ngali Khola Valley, 2000-2300 m, 15.V.2005, leg. J. Schmidt (6, NKME); Kathmandu, Shivapuri Lekh, slope W of Bagmati river, 2000-2300 m, 22-23.V.2005, leg. J. Schmidt (1, NKME); Dhaulagiri Himal, $\mathrm{N}$ Banduk vill., S-slope, $28^{\circ} 27^{\prime} 22^{\prime \prime} \mathrm{N}, 83^{\circ} 35^{\prime} 13^{\prime \prime} \mathrm{E}$ to $28^{\circ} 28^{\prime} 07^{\prime \prime} \mathrm{N}, 83^{\circ} 35^{\prime} 10^{\prime \prime} \mathrm{E}, 1900-2300 \mathrm{~m}$, 6.V.2009, leg. J. Schmidt (4 §’, 5 ㅇ, NKME); Kaski distr., above Pothana, 2000 m [ $28^{\circ} 19^{\prime} \mathrm{N}$, 8350’E], 27-29.IV.1995, leg. Martens \& Schawaller (426) (1, SMNS); INDIA: N-, Uttaranchal

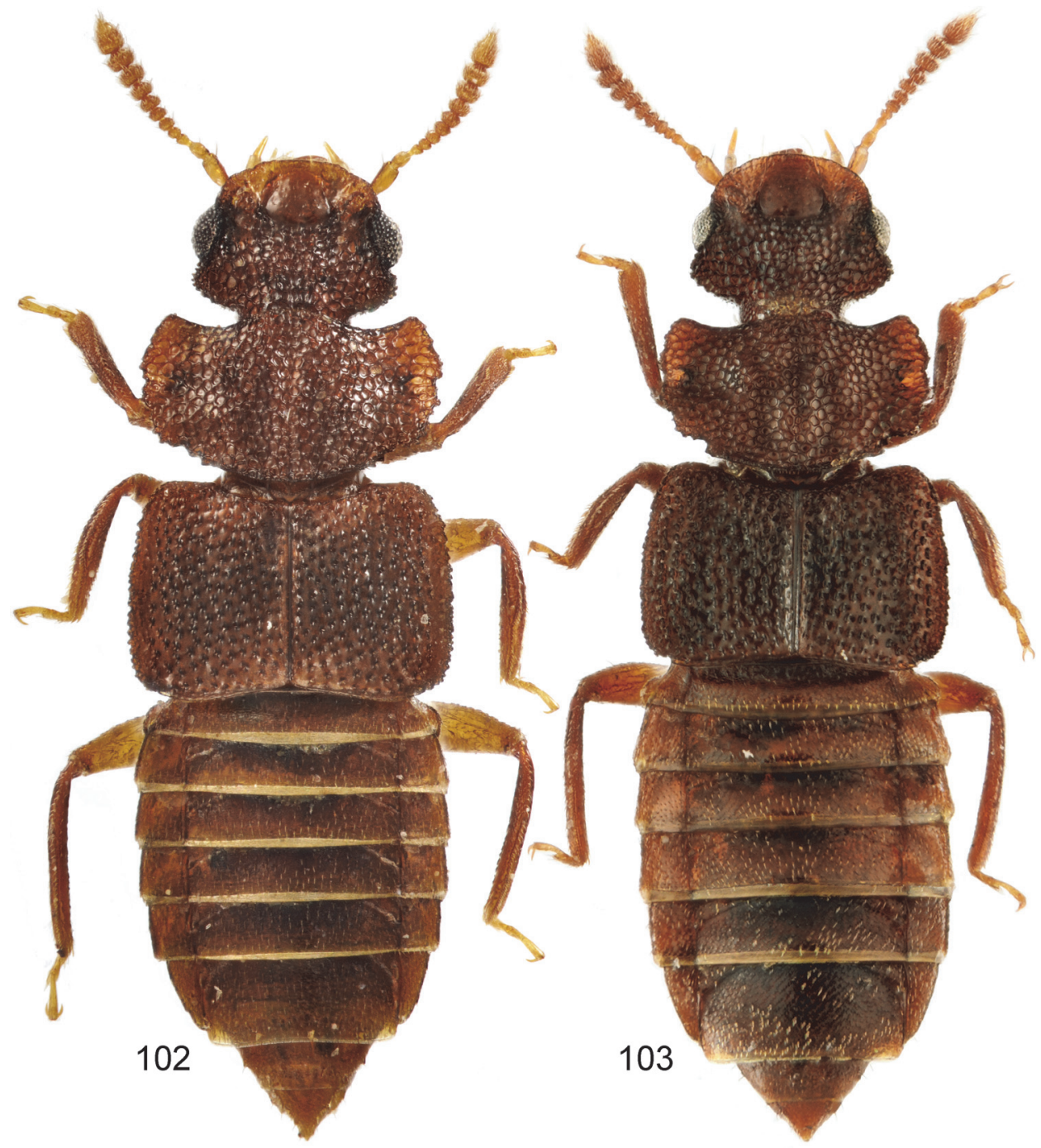

Figs 102-103. Habitus of Anotylus species. $102=$ A. genalis (Fauvel, 1904), $103=$ A. anguliceps (Cameron, 1934) 


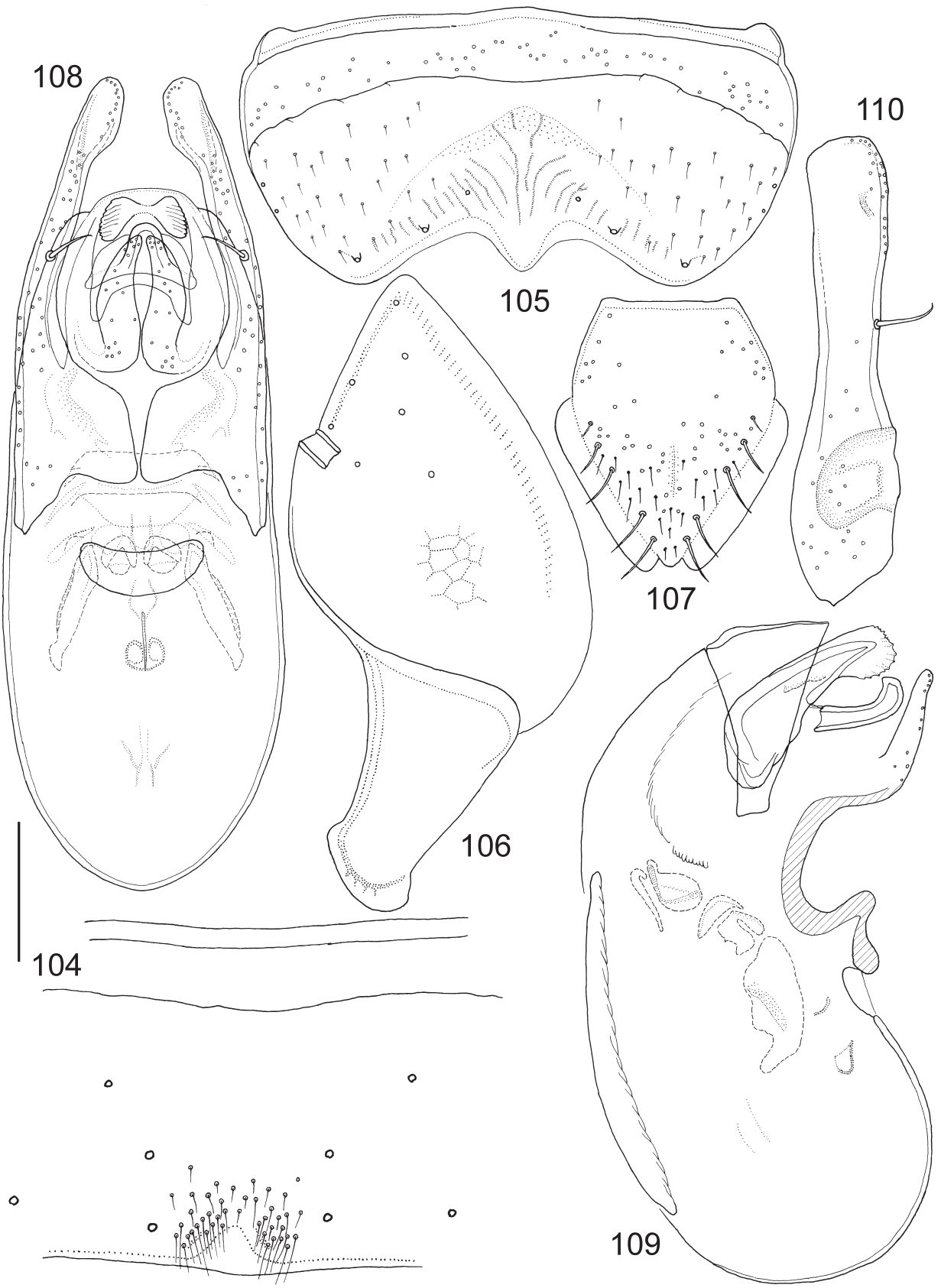

Figs 104-110. Anotylus franzi (Coiffait, 1982), male. $104=$ median part of sternite VII, $105=$ sternite VIII, 106 = tergite IX, 107 = tergite $X, 108$ = aedeagus, frontal view, $109=$ median lobe, lateral view, 110 = paramere, lateral view. Scales: $0.10 \mathrm{~mm}$ for Figs 108-110, $0.12 \mathrm{~mm}$ for Figs 104, 106-107, 0.13 mm for Fig. 105 


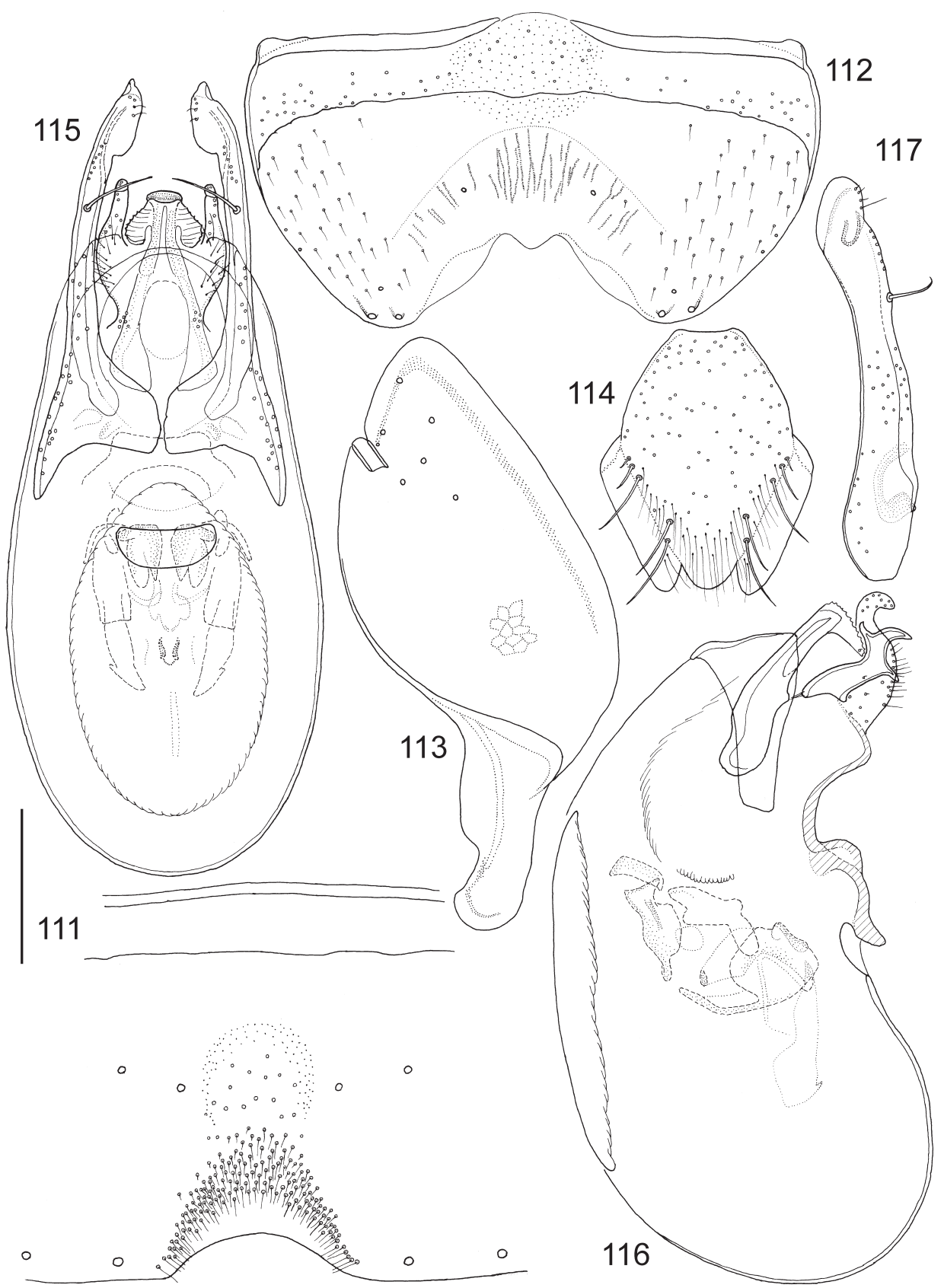

Figs 111-117. Anotylus ganapati sp. n., male. $111=$ median part of sternite VII, $112=$ sternite VIII, $113=$ tergite IX, $114=$ tergite $X, 115=$ aedeagus, frontal view, $116=$ median lobe, lat eral view, 117 = paramere, lateral view. Scales: $0.18 \mathrm{~mm}$ for Fig. 111, $0.20 \mathrm{~mm}$ for Figs 112, 114-117, $0.28 \mathrm{~mm}$ for Fig. 113 
state, Nainital, China Peak env., 1900-2300 m, 18-19.VII.2003, leg. Z. Kejval \& M. Tryzna (2, NHMW, 1, MHNP, 1, SDEI); Assam, North Cachar Hills distr., Mt. Borail, trail Borail Peak - Notun Leikul, $25^{\circ} 06^{\prime} 47^{\prime \prime} \mathrm{N}, 93^{\circ} 03^{\prime} 05^{\prime \prime} \mathrm{E}, 1650 \mathrm{~m}, 20 . \mathrm{X} .2005$, leg. G. Cuccodoro \& A. Marletta (9c), sifting moist leaf litter in forest with Fagaceae (1, MHNG); Meghalaya, East

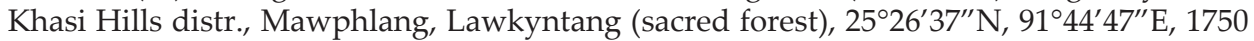
m, 23.X.2004, leg. G. Cuccodoro, C. Carlton, R. Leschen \& D. Errne (11a), in Quercus + Magnolia forest, sifting leaf litter and nuts on flat area near dry stream (5, MHNG); CHINA: Guizhou, Leishan Co, SE Kaili, NE Leishan, Leigong Shan, E-slope, ca. $2.5 \mathrm{~km}$ E of pass between Leishan and Fangxiang village, $26^{\circ} 23.39^{\prime} \mathrm{N}, 108^{\circ} 13.33^{\prime} \mathrm{E}$, ca. $1600 \mathrm{~m}, 23-24$. VI.2001, leg. H. Schillhammer (17a), sifted from semi-dry leaf litter with plenty of wooden components (2 0,5 , NHMW); Guizhou, Leishan Co, SE Kaili, NE Leishan, Leigong Shan, E-slope, ca. $2.5 \mathrm{~km}$ E of pass, $26^{\circ} 23.39^{\prime} \mathrm{N}, 108^{\circ} 13.33^{\prime} \mathrm{E}$, ca. $1600 \mathrm{~m}, 19 . \mathrm{VI} .2001$, leg. H. Schillhammer (14), sifted from mouldy debris of leaves and branches in very dark portion of forest $\left(1 \delta^{\lambda}, 11\right.$ ㅇ, NHMW, $1 \delta^{\lambda}$, IZAS); VIETNAM: N-, pass $8 \mathrm{~km} \mathrm{NW}$ Sa Pa, $22^{\circ} 21^{\prime} 13^{\prime \prime} \mathrm{N}$, $103^{\circ} 46^{\prime} 01^{\prime \prime} \mathrm{E}, 2030 \mathrm{~m}, 10 . \mathrm{VIII} .2015$, leg. V. Assing (10a) sifted from litter and roots under bushes near the margin of a secondary deciduous forest ( 1 , coll. Assing).

Description - Measurements (in mm, $\mathrm{n}=10)$ : $\mathrm{HW}=0.75(0.70-0.79)$; $\mathrm{TW}=0.72(0.67-$ $0.76) ; \mathrm{PW}=1.01$ (0.92-1.06); $\mathrm{EW}=1.11$ (1.04-1.18); $\mathrm{AW}=1.17$ (1.10-1.22); $\mathrm{HL}=0.57$ (0.53$0.60) ; \mathrm{EL}=0.16(0.15-0.17) ; \mathrm{TL}=0.22(0.20-0.24) ; \mathrm{PL}=0.68(0.63-0.73) ; \mathrm{SC}=0.73(0.69-0.78)$; $\mathrm{FB}=2.16(2.04-2.22) ; \mathrm{BL}=3.97$ (3.87-4.06). Habitus as in Fig. 72. Forebody weakly shining because of sculpture, abdomen roughly and rather densely but shallowly punctured, interspaces shiny, more lustrous than forebody. Forebody, abdomen and first antennomere blackish dark brown with slight reddish tint, more reddish along pronotal side margins and anterior margin of head. Legs, mouthparts and rest of antennae reddish dark brown. Head with anterior margin possessing rim (weaker in middle), evenly arched in middle, but laterally more truncate, angled at supraantennal prominences, these as moderately elevated oblique ridges, rim continuing posteriad along inner border of eyes, disc slightly impressed beside it. Epistomal suture forming subcircular impression (less sculptured inside), upper surface with weak tactile setae (intratemporalis) situated postero-medially of each eye. Eyes medium-sized and slightly bulging, in dorsal view temples $(1.4 \times$ length of eye) alatiform, laterally rounded and posteriorly constricted to form distinct neck, separated by transversal occipital furrow. Antennomere 1 flattened club-shaped and strongly microsculptured, segment 2 oval, segment 3 club-shaped, segment 4 small and slightly elongate, segments 5-11 with basal dishes, articles 6-10 very slightly transverse, segment 11 somewhat lighter. Head and pronotum with areolate sculpture, along the midline somewhat costulate (composed of fine, short, elevated lines). Pronotum with lateral portion explanate, edge alatiform with very sparse short setae; lateral border evenly arched with thin marginal ridge and a number of small, irregularly distributed denticles, posterior margin evenly arched; disc medially with two faint and posteriorly confluent longitudinal ridges, slightly impressed alongside them, and near side margin. Elytra with a thinning margin posteriorly, continuing on lateral part. Elytral epipleural ridge apparent as stronger sculpture lines in lateral alatiform portions; shoulders well developed, surface foveolate and in between punctures torulose. Legs short, pro- and mesotibia with several spinulose rows, metatibia with longitudinal ctenidium of spinules in distal half. Abdomen with sides moderately arcuate, second segment with paratergites broadening posteriorly (abdomen slightly constricted at base), those of segments III-VII thin, mesal paratergites broad; posterior edge of tergite VII with thin palisade fringe, median part of sternite VII as 
in Fig. 111, sternite VIII (Fig. 112), tergites IX (Fig. 113), X (Fig. 114) and aedeagus as in Figs 115-117, spermatheca as in Fig. 266.

Etymology - The species is named after Ganapati, another Sanskrit name of Ganesha, the elephant-headed god in Hinduism (noun in apposition).

Distribution - Appears to be a rather widespread species from northern India and Nepal to southern China. A single female specimen is known from Vietnam, but its identification requires verification by male exemplars.

Remarks - A series of the available specimens was obtained by sifting from mouldy debris of leaves and branches and wooden components in a very dark part of forest.

\section{Anotylus genalis (Fauvel, 1904) \\ (Figs 102, 118-124)}

Oxytelopsis genalis Fauvel, 1904: 96; Cameron 1928: 401; Herman 1970: 413; Hammond 1984: 200, Herman 2001: 1414.

Examined type material - Oxytelopsis genalis Fauvel - Holotype (†): “Lecto-; type [lilac framed disc, curator label] \Perak; Malacca \genalis; Fvl. \Oxytelopsis; genalis Fvl. \Coll. et det. A. Fauvel; Oxytelopsis; genalis; Fauv.; R.I.Sc.N.B. $17.479 \backslash$ Syntype \ P. M. Hammond; det. 1972; Lectotype [sic] \Anotylus; genalis (Fauvel); det. Makranczy, 2015" (ISNB).

Other material - MALAYSIA: Pahang distr., $30 \mathrm{~km}$ NE Raub, Laba Lembik, $3^{\circ} 56^{\prime} \mathrm{N}$, 101³8'E, 300 m, 22.IV-15.V.2002, leg. E. Jendek \& O. Šauša (06-21) (1, NMPC); Pahang, Pulau Tioman, Kampung Juara, 12.III.1995, leg. O. Merkl (no. 23), secondary growth, at light (1, HNHM); Perak, Cameron Highlands, cascade Sungei Simei, 28.III.1977, leg. T. Jacoud (85i) (13, MHNG); Perak, Cameron Highlands, cascade Sungei Simei, 25.III.1977, leg. T. Jacoud (34i, 61i) (35, MHNG); Ulu Gombar nr. Kuala Lumpur ca. $800 \mathrm{ft}\left[3^{\circ} 14^{\prime} 10^{\prime \prime} \mathrm{N}\right.$, 1014'00"E], 11-14.VII.1968, leg. R.W. Taylor (6, MHNG); W-, Lake Kenyir, 5 km SW dam, 50 km SW Kuala Terengganu 350 m, 7-12.VII.2001, leg. A. Schulz \& K. Vock (10, SMNS); Federal Malay State, Selangor, Bukit Kutu, leg. M. Cameron (1, MHNG); Johore, Gunung Pulai, near foot, ca. 500 ft, 22.V.1968, leg. R.W. Taylor (68/44b), rainforest, leafmould berlesate (1, ANIC); Penang Island, Penang Hill, near summit, 700 m, 9.XI.1999, leg. G. Cuccodoro \& I. Löbl (2b), broadleaf evergreem forest, sifting forest leaf litter (1, MHNG); Penang Island, Penang Hill, 650 m, 19.XI.1999, leg. G. Cuccodoro \& I. Löbl (10a), broadleaf evergreen forest, forest leaf litter and vegetational debris on trail (1, MHNG); MALAYSIA/ BORNEO: Sarawak, Kubah N. P., 200-400 m, 6-8.III.2008, leg. W. Schawaller (1, SMNS); Sabah, Kinabalu N. P., Sayap, 1000 m, 25.XI.1996, leg. D. Grimm, at light (1, SMNS); Sabah, Batu, Punggul Resort env., 24.VI-1.VII.1996, leg. J. Kodada \& F. Čiampor (11c), vegetation debris and forest floor litter accumulated around large trees near river (11, MHNG); Sabah, Sepilok Forest Res. (near Sandakan), 8.VI.1968, leg. R.W. Taylor, ex humus (1, MHNG); Sabah, Tawau, Quoin Hill, 750 ft, 16-19.VI.1969, leg. R.W. Taylor (1, MHNG), same but VI.1968, ex humus (1 §, 1 †, MHNG); Sabah, Crocker Range N. P., NW Keningau, 9001200 m, 18.XI.1996, leg. D. Grimm, at light (1, SMNS); Sabah, Crocker Range N. P., NW Keningau, 900-1200 m, 16-20.XI.1996, leg. W. Schawaller (1, SMNS); Sabah, Mt. Kinabalu 
National Park, above Poring Hot Springs, 520 m, 15.VIII.1988, leg. A. Smetana (B115), sifting of layers of fermenting fruits (of a tree, Ficus sp.) on forest floor and debris under (375, FMNH, $1 \hat{\jmath}, 1$ \&, NIBR, $1 \hat{\jmath}$, IZAS, $1 \hat{\jmath}$, HNHM); Sabah, Mt. Kinabalu National Park, Poring Hot Springs, 495 m, 21.VIII.1988, leg. A. Smetana (B136), sifting of layers of fermenting fruits (of a tree, Ficus sp.) on forest floor and debris under (16, FMNH); Sabah, Mt. Kinabalu National Park, above Poring Hot Springs, 520 m, 22.VIII.1988, leg. A. Smetana (B139), sifting of layers of rotting fruits (Ficus sp.) on forest floor and debris under (19, FMNH); Sabah, Mt. Kinabalu National Park, Poring Hot Springs, 495 m, 23.VIII.1988, leg. A. Smetana (B140), sifting of layers of fallen fruits in primary tropical rain forest (20, FMNH), same but 24.VIII.1988 (B144) (33, FMNH, 1 ㅇ, HNHM, 1 , , IZAS, 1 , MNHP, 1, ZMUC); Sabah, Mt. Kinabalu National Park, Poring Hot Springs, 485 m, 25.VIII.1988, leg. A. Smetana (B147), sifting of accumulated old fruits of Areca palms, including debris and humus underneath, open area (1, FMNH), same but 14-31.VIII.1988 (B166) (1, FMNH); Sabah, Mt. Kinabalu National Park, Poring Hot Springs, 495 m, 30.VIII.1988, leg. A. Smetana (B161), sifting of three large fermenting fruits (cocoa nut size) and debris under them in rain forest

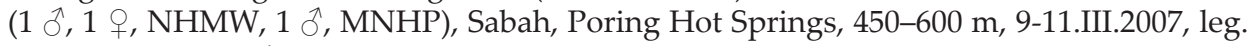
W. Schawaller (1 $\hat{\delta}$, SMNS); Sabah, Kinabalu Park, Poring Hot Springs, ca. 500 m, forêt à Dipterocarpaceae relativement sèche, 6.V.1987, leg. D. Burckhardt \& I. Löbl (14a), tamisage de bois pourri avec champignons a la lisière de la forêt, et de feuilles mortes au pied des arbres la forêt (2, MHNG); Sabah, Poring Hot Springs, 500 m env., forêt de Dipterocarpaceae, 7.V.1987, leg. D. Burckhardt \& I. Löbl (15a), tamisage dans la forêt (3, MHNG); Sabah, Poring Hot Springs, 500 m env., forêt de Dipterocarpaceae, 11.V.1987, leg. D. Burckhardt \& I. Löbl (21), tamisage au pied de vieux arbres et sur des champignons, à proximité d'un ruisseau (1, MHNG); Sabah, Poring Hot Springs, 500 m env., forêt de Dipterocarpaceae à proximité des "Headquarters", 13.V.1987, leg. D. Burckhardt \& I. Löbl (24a), tamisage de bois pourri et de feuilles mortes (1, MHNG); BRUNEI: Temburong, Kuala Belalong (East), 13.II.1995, leg. Borcherding (1 $\hat{\jmath}$, coll. Assing); THAILAND: prov. Chiang Mai, Doi Inthanon, 1080 m, 24.II.1987, leg. P. Schwendinger (2, MHNG); prov. Chiang Mai, Doi Chiang Dao, 450 m, 7.V.1987, leg. P. Schwendinger (1, MHNG); VIETNAM: Ninh Binh Prov., Res. Cuc-Phüöng, 200 m, 14.X.1963, leg. T. Pócs, dans forêt tropic. pluv., à la lumière (1 §̃, HNHM), same but 15.V.1966, leg. Gy. Topál (no. 368), singled (1, HNHM); PHILIPPINES/LUZON: Lagunas (Calabarzon), Mt. Banahaw above Kinabuhayan, 600-700 m, trail to Crystalino, 24.XI.1995, J. Kodada \& B. Rigová, degraded rainforest, forest floor litter (1, MHNG); Lagunas (Calabarzon), Mt. Banahaw above Kinabuhayan, 600-700 m, trail to Crystalino, 24.XI.1995, leg. I. Löbl (5a), degraded rainforest, forest floor litter (2, MHNG); Central Luzon, Mt. Banahaw above Kinabuhayan, summit trail, ca. 800 m, 25.XI.1995, leg. I. Löbl (6), degraded rainforest, on slope (3, MHNG); PHILIPPINES/MINDANAO: $30 \mathrm{~km}$ NW Maramag, Bagongsilang, 1700 m, 13-17.V.1996, leg. Bolm (1, SMNS); INDONESIA/ SUMATRA: Lampung prov., Bukit Barisan Selatan N.P., road Krui-Liwa 2 km SW Kubuprahu, 480-540 m, 4.III.2000, leg. P. Schwendinger (Sum-00/25), disturbed evergreen rainforest and secondary forest, sifting (1, MHNG); Jambi, $\mathrm{km} 8$ on road Sungaipenuh to Tapan, 1200 m, 9.XI.1989, leg. I. Löbl, D. Agosti, D. Burckhardt (8), sifting of vegetational debris of trees and bamboos in ravine (13, MHNG, $1 \hat{\jmath}, \mathrm{HNHM}$ ); Jambi, km 12 on road Sungaipenuh to Tapan, 1350 m, 9.XI.1989, leg. I. Löbl, D. Agosti, D. Burckhardt (9), sifting of leaf litter and moldy branches in secondary scrub (7, MHNG); Jambi, km 15 on road from Sungaipenuh to Tapan, 1450 m, 9.XI.1989, leg. I. Löbl, D. Agosti, D. Burckhardt (10), sifting of vegetational debris in degraded montane Lithocarpus-Castanopsis forest (1, MHNG); Jambi, Mt. Kerinci, 1750-1850 m, 11-12.XI.1989, leg. I. Löbl, D. Agosti, D. Burck- 
hardt (11), sifting of vegetational debris in montane Lithocarpus-Castanopsis forest (1, MHNG); West Sumatra prov., Lake Maninjau, waterfall ab. Gasang, 720 m, 9.II.2000, leg. P. Schwendinger (Sum-00/04), sifting of rotten bamboos (26, MHNG); West Sumatra, Anai Valley Nature Reserve, 10 km W Padangpanjan, 250 m, 17.XI.1989, leg. I. Löbl, D. Agosti, D. Burckhardt (18), sifting of vegetational debris in degraded lowland forest (1, MHNG); West Sumatra, Padangpanjan, 600 m, 17.XI.1989, leg. I. Löbl, D. Agosti, D. Burckhardt (20), sifting of rotten bamboos (28, MHNG); West Sumatra, Palopo Nature Reserve, N Bukittingi, 900 m, 18-20.XI.1989, leg. I. Löbl, D. Agosti, D. Burckhardt (21), sifting of vegetational debris in secondary forest on steep slope (13, MHNG); West Sumatra, Panti, 250 m, 19.XI.1989, leg. I. Löbl, D. Agosti, D. Burckhardt (23), sifting of vegetational debris in lowland swamp forest (3, MHNG); West Sumatra, Bukittingi, Panti, 700 m, 25-26.X.1991, leg. A. Riedel, (1 0 , 2 + , SMNS); Aceh, Mt. Leuser National Park, Ketambe Research Station, 300$500 \mathrm{~m}$, lowland dipterocarp forest, 23-30.XI.1989, leg. I. Löbl, D. Agosti, D. Burckhardt (25a), sifting of vegetational debris (15, MHNG); North Sumatra, $5 \mathrm{~km} \mathrm{~W} \mathrm{Brastagi,} \mathrm{Tong-}$ koh, 1450 m, mixed pine forest with many epiphytes, 3.XII.1989, leg. I. Löbl, D. Agosti, D. Burckhardt (29a), sifting of vegetational debris (7, MHNG); INDONESIA/JAVA: West Java, Cibodas Botanical Garden, near Cipanas, ca. 50 km E Bogor, 1400 m, 3-6.XI.1989, leg. I. Löbl, D. Agosti, D. Burckhardt (2a), sifting of vegetational debris in montane LithocarpusCastanopsis forest (24, MHNG); Bandung, Maribaya, 22 km NE Bandung, 27.VII.1984, leg. J. Robert (19 and 20a), Winkler extraction (13, MHNG); Col du Puncak et Cibodas, lac Telaga Warna, 4.VIII.1984, leg. J. Robert (26), Winkler extraction (3, MHNG); West Java, Cibodas Botanical Garden, near Cipanas, ca. 50 km E Bogor, 1400 m, 3-6.XI.1989, leg. I. Löbl, D. Agosti, D. Burckhardt (2a), sifting of vegetational debris in montane Lithocarpus-Castano-

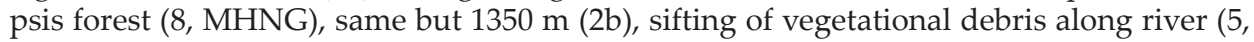
MHNG); C-Java, N slopes of Dieng plateau, Petungkriyono, mountain N Tinalum, 0706.418'S, 109²4.514'E, 1115 m, 22.VIII.2006, leg. A. Riedel (sample 1), sifted, (1 $\hat{\delta}$, SMNK); INDONESIA/BORNEO: Kalimantan Barat, Gunung Palung National Park, Cabang Panti research site, $1^{\circ} 13^{\prime} \mathrm{S}, 110^{\circ} 07^{\prime} \mathrm{E}$, lowland rainforest, 18-26.VII.1993, leg. O. Merkl (9), at light (14, HNHM); Kalimantan Barat, Bajas logging area above Samanja, $1^{\circ} 13^{\prime} \mathrm{S}, 110^{\circ} 06^{\prime} \mathrm{E}$, clearing of lowland rainforest, $27 . V I I .1993$, leg. O. Merkl (23), at light (1 ${ }^{\prime}$, 21, HNHM).

Redescription - Measurements (in mm, $\mathrm{n}=10)$ : $\mathrm{HW}=0.62(0.59-0.65)$; TW $=0.60(0.57-$ $0.64) ; \mathrm{PW}=0.88(0.82-0.98) ; \mathrm{EW}=0.97(0.92-1.14) ; \mathrm{AW}=0.94(0.89-1.06) ; \mathrm{HL}=0.45(0.41-$ $0.48) ; \mathrm{EL}=0.15(0.14-0.16) ; \mathrm{TL}=0.15(0.14-0.17) ; \mathrm{PL}=0.52$ (0.48-0.59); $\mathrm{SC}=0.59$ (0.55-0.66); $\mathrm{FB}=1.63(1.50-1.79) ; \mathrm{BL}=2.99(2.72-3.31)$. Habitus as in Fig. 102. Forebody only slightly shining for strong sculpture, abdomen very finely microsculptured (punctation only in traces), rather dull. Whole body reddish medium to dark brown, antennae and mouthparts somewhat lighter, reddish medium brown. Head with an extremely thin rim on anterior margin, latter more or less truncate in middle, slightly angled also at supraantennal prominences, these as moderately elevated oblique ridges, disc slightly impressed near eyes, middle of vertex slightly elevated. Epistomal suture forming subcircular impression (shiny inside), upper surface without tactile setae, eyes rather large, moderately convex; in dorsal view temples (same length as eye) alatiform, laterally bluntly angled and posteriorly constricted to form distinct neck, however, dorsal side not separated by occipital furrow, rather by dorsum of neck bearing microsculpture instead of punctation. Antennomere 1 flattened club-shaped and finely microsculptured, segment 2 broad at base and narrowing towards stout club-shaped segment 3, segment 4 slightly transverse, segments 5-11 with 


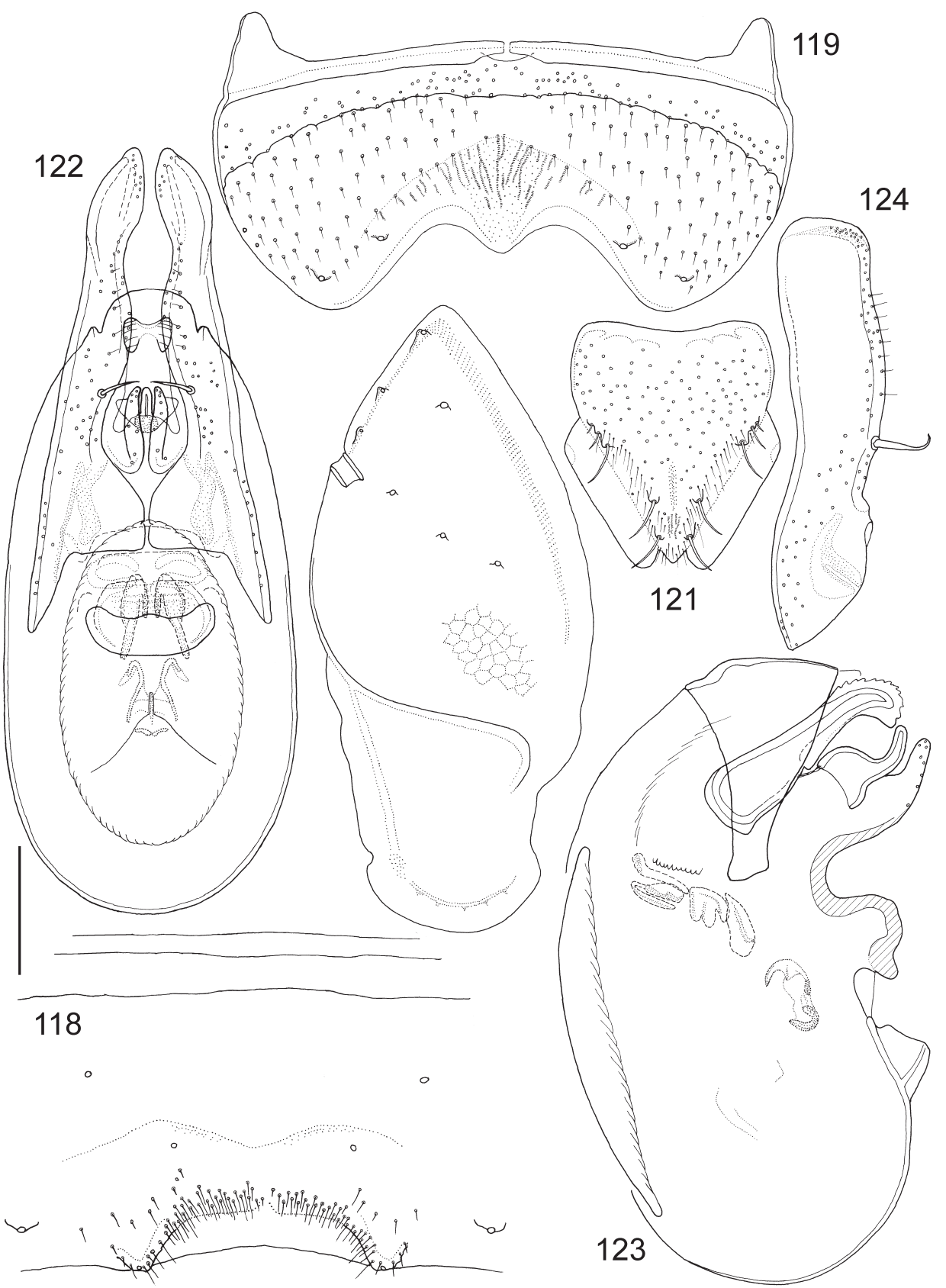

Figs 118-124. Anotylus genalis (Fauvel, 1904), male. $118=$ median part of sternite VII, $119=$ sternite VIII, $120=$ tergite IX, $121=$ tergite $X, 122=$ aedeagus, frontal view, $123=$ median lobe, lateral view, 124 = paramere, lateral view. Scales: 0.10 mm for Figs 118, 122-124, 0.12 mm for Figs 120-121, 0.14 mm for Fig. 119 
basal dishes, segments 6-10 transverse (about $1.5 \times$ broader than long). Head and pronotum with mostly alveolate sculpture. Pronotum with lateral portion explanate and slightly upturned, edge alatiform with sparse short setae; lateral border uneven and with a number of small, irregularly distributed denticles, posterior margin slightly incised before corners; disc medially with two parallel and posteriorly almost confluent longitudinal ridges, posteriorly impressed alongside them, laterally impressed around middle of sides. Elytra with membranous posterior margin extending from sutural corner to 5/6 of hind margin. Elytral epipleural ridge present with up-turned lateral alatiform portions, disc longitudinally impressed alongside; shoulders well developed, surface foveolate, in between punctures torulose. Legs short, pro- and mesotibia with several spinulose rows, metatibia with longitudinal ctenidium of spinules in distal half. Abdomen with sides gently arcuate, second segment with paratergites strongly broadening posteriorly (abdomen appearing constricted at base), those of segments III-VII thin, mesal paratergites broad; posterior edge of tergite VII with thin palisade fringe, median part of sternite VII as in Fig. 118, sternite VIII (Fig. 119), tergites IX (Fig. 120), X (Fig. 121) and aedeagus as in Figs 122-124.

Distribution - This species occurs from Thailand and Vietnam to the Greater Sunda Islands (Sumatra, Java, Borneo) and the Philippines.

Remarks - The taxon was described from a "unique" (specimen), therefore it is the holotype. This species obviously prefers various fermenting fruits of tropical trees as was frequently collected in such habitat. A strange form of this species exists with conspicuously convex pronotal disc (pronotal length also slightly exceeding that of normal specimens). Examination of male genitalia and terminalia did not reveal any consistent difference.

\section{Anotylus gunung sp. n.}

(Figs 75, 125-131)

Type material - Holotype (ふ̋): THAILAND: S-, Yala prov., Betong distr., Gunung Cang

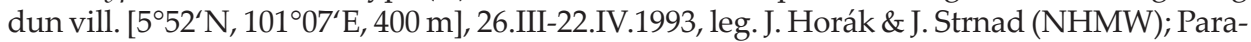
types (3): same data as holotype (1 $\delta$, MHNG); INDIA: Meghalaya, $1 \mathrm{~km}$ E Tura, 500-600

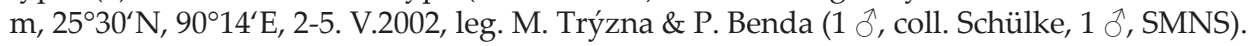

Description - Measurements (in mm, $\mathrm{n}=4)$ : $\mathrm{HW}=0.56(0.54-0.57)$; $\mathrm{TW}=0.53(0.51-$ $0.54) ; \mathrm{PW}=0.68(0.65-0.70) ; \mathrm{EW}=0.77(0.73-0.81) ; \mathrm{AW}=0.77(0.72-0.82) ; \mathrm{HL}=0.41(0.40$ $0.42) ; \mathrm{EL}=0.13(0.12-0.13) ; \mathrm{TL}=0.17(0.16-0.17) ; \mathrm{PL}=0.47(0.44-0.49) ; \mathrm{SC}=0.53(0.51-0.55)$; $\mathrm{FB}=1.50(1.44-1.54) ; \mathrm{BL}=2.81$ (2.57-2.95). Habitus as in Fig. 75. Forebody rather dull for dense sculpture, abdomen finely punctate and coriaceous microsculptured, somewhat more lustrous. Head and first antennomeres blackish dark brown, rest of body reddish dark brown, anterior margin of head often lighter, reddish. Head with anterior margin possessing marked rim (weaker in middle), evenly arched but slightly angled at supraantennal prominences, these as moderately elevated oblique ridges, rim continuing posteriad along inner border of eyes, disc slightly impressed beside it. Epistomal suture forming obsolete circular impression (sculpture inside not differing), upper surface with tactile setae (intratemporalis) situated postero-medially of each eye. Eyes medium-sized and slightly bulging, in dorsal view temples (1.3× length of eye) alatiform, laterally rounded and posteriorly constricted to form distinct neck, separated by transversal occipital furrow. Anten- 


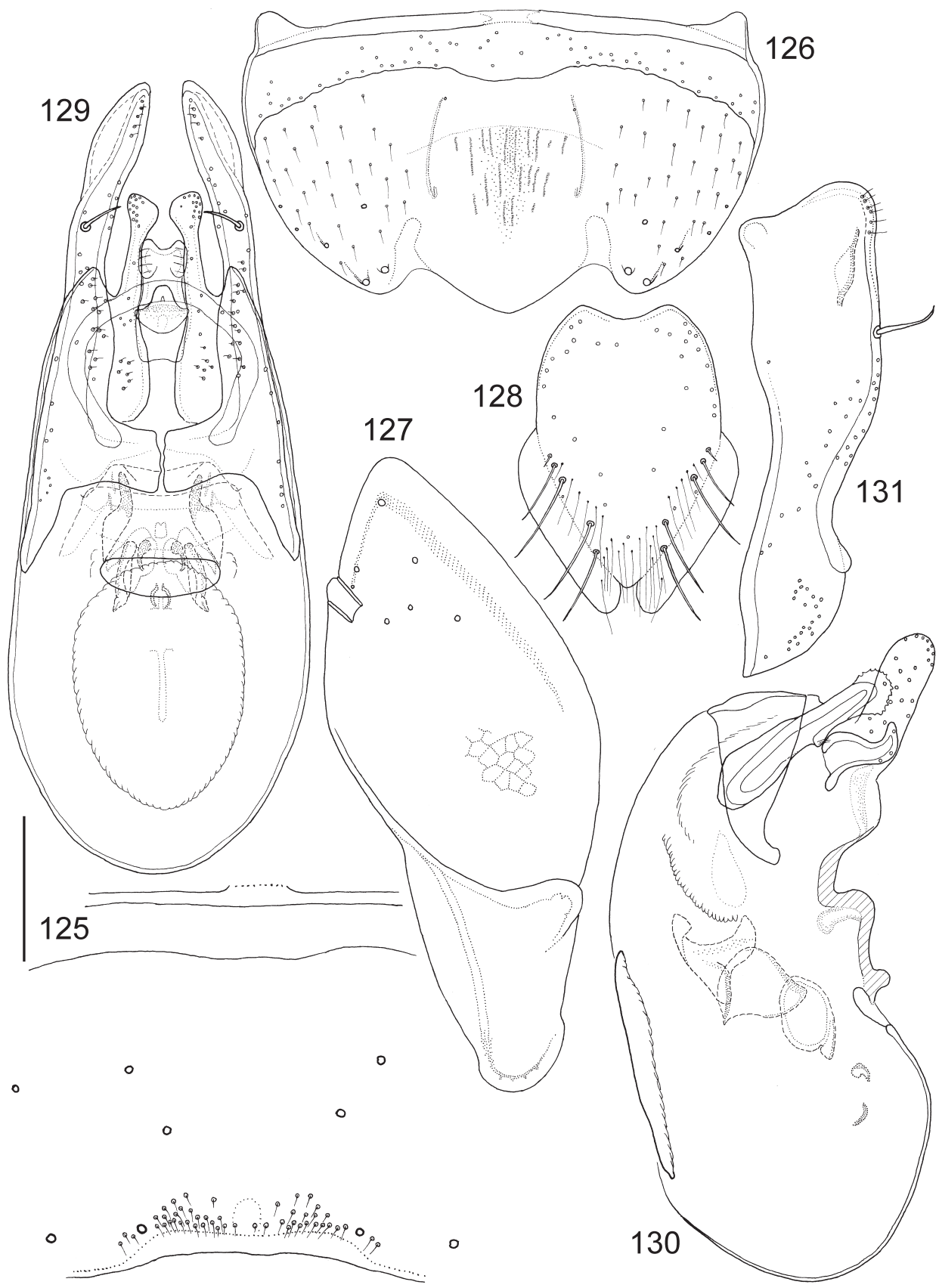

Figs 125-131. Anotylus gunung sp. n., male. $125=$ median part of sternite VII, $126=$ sternite VIII, 127 = tergite IX, 128 = tergite $X, 129=$ aedeagus, frontal view, $130=$ median lobe, lateral view, 131 = paramere, lateral view. Scales: $0.10 \mathrm{~mm}$ for Figs $129-131,0.11 \mathrm{~mm}$ for Fig. 128, $0.125 \mathrm{~mm}$ for Figs 125, 127, $0.14 \mathrm{~mm}$ for Fig. 126 
nomere 1 flattened club-shaped and strongly microsculptured, segment 2 basally broad ovoid, segment 3 club-shaped, segment 4 small and slightly transverse, segments 5-11 with basal dishes, articles $6-10$ slightly transverse (about $1.2 \times$ broader than long). Head and pronotum with lacunose sculpture, in midline transitional to costulate. Pronotum with lateral portion explanate, edge alatiform with very sparse short setae; lateral border almost evenly arched with thin marginal ridge and a number of small, irregularly distributed denticles, posterior margin evenly arched, posterior corners weakly pronounced; disc medially with two nearly parallel longitudinal ridges, posteriorly impressed alongside them, laterally somewhat impressed near side margin. Elytra without membranous posterior margin. Elytral epipleural ridge apparent as stronger sculpture lines in lateral alatiform portions, disc with very slight to no impression in centre; shoulders well developed, surface obscurely foveolate, elevations of interspaces conflent as short longitudinal ridges. Legs short, pro- and mesotibia with several spinulose rows, metatibia with longitudinal ctenidium of spinules in distal half. Abdomen with sides moderately arcuate, second segment with paratergites broadening posteriorly (abdomen slightly constricted at base), those of segments III-VII thin, mesal paratergites broad; posterior edge of tergite VII with thin palisade fringe, median part of sternite VII as in Fig. 125, sternite VIII (Fig. 126), tergites IX (Fig. 127), X (Fig. 128) and aedeagus as in Figs 129-131.

Etymology - The name is from "gunung" (often abbreviated as Gn., meaning mountain range) in local language (noun in apposition).

Distribution - The species is known from two pairs of specimens collected at rather distant localities in southern Thailand and the Indian state Meghalaya, respectively.

Remarks - A rather enigmatic and small species, appears to be most closely related to the much larger $A$. coonoor.

\section{Anotylus hartmanni sp. n.}

(Figs 82, 132-138, 267)

Type material - Holotype (§): NEPAL: Manaslu Mts., SE-slope, W Gupchi Danda, 2200-2300 m [28 $\left.08^{\prime} 37^{\prime \prime} \mathrm{N}, 84^{\circ} 44^{`} 42^{\prime \prime} \mathrm{E}\right], 28 . V .2006$, leg. J. Schmidt, sifting leaf litter in forest remains along mountain crest (NKME); Paratypes (41): same data as holotype $(1 \hat{\sigma}$, NHMW); Manaslu Mts., Dudh Pokhari Lekh, below Helam Pokhari, 2000 m [ $28^{\circ} 11^{`}$ N,

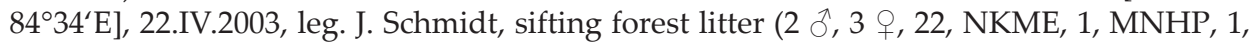
ZMUC, 1, ISNB, 1, NMPC, 1, CNCI, 1, FMNH, 1, AMNH, 1, MHNG, 1, SDEI, 1, SMNS, 1, NIBR, 1 ô, BMNH, 1 ô, HNHM); NEPAL: Manaslu Mts., Dudh Pokhari Lekh, upper Phulinagiri, Madi, $2500 \mathrm{~m}$ [above Phulingiri Madi, $28^{\circ} 13^{`} \mathrm{~N}, 84^{\circ} 34^{\prime} \mathrm{E}$ ], 19-21.IV.2003, leg. J. Schmidt, sifting forest litter (1, NKME).

Description - Measurements (in mm, $\mathrm{n}=10)$ : $\mathrm{HW}=0.63(0.60-0.65) ; \mathrm{TW}=0.65(0.61-$ $0.68) ; \mathrm{PW}=0.90$ (0.85-0.97); $\mathrm{EW}=0.90$ (0.84-0.95); $\mathrm{AW}=0.97(0.90-1.03) ; \mathrm{HL}=0.48$ (0.45$0.51) ; \mathrm{EL}=0.11(0.10-0.12) ; \mathrm{TL}=0.21(0.20-0.23) ; \mathrm{PL}=0.57(0.53-0.60) ; \mathrm{SC}=0.48(0.45-0.50)$; $\mathrm{FB}=1.58(1.49-1.65) ; \mathrm{BL}=3.30$ (2.61-3.50). Habitus as in Fig. 82. Forebody moderately shining despite sculpture, abdomen with faint coriaceous microsculpture, shallowly punctured, as lustrous as forebody. Body reddish dark brown except abdomen, anterior margin of head, lateral margin of pronotum, antennae and mouthparts medium to dark brown. 


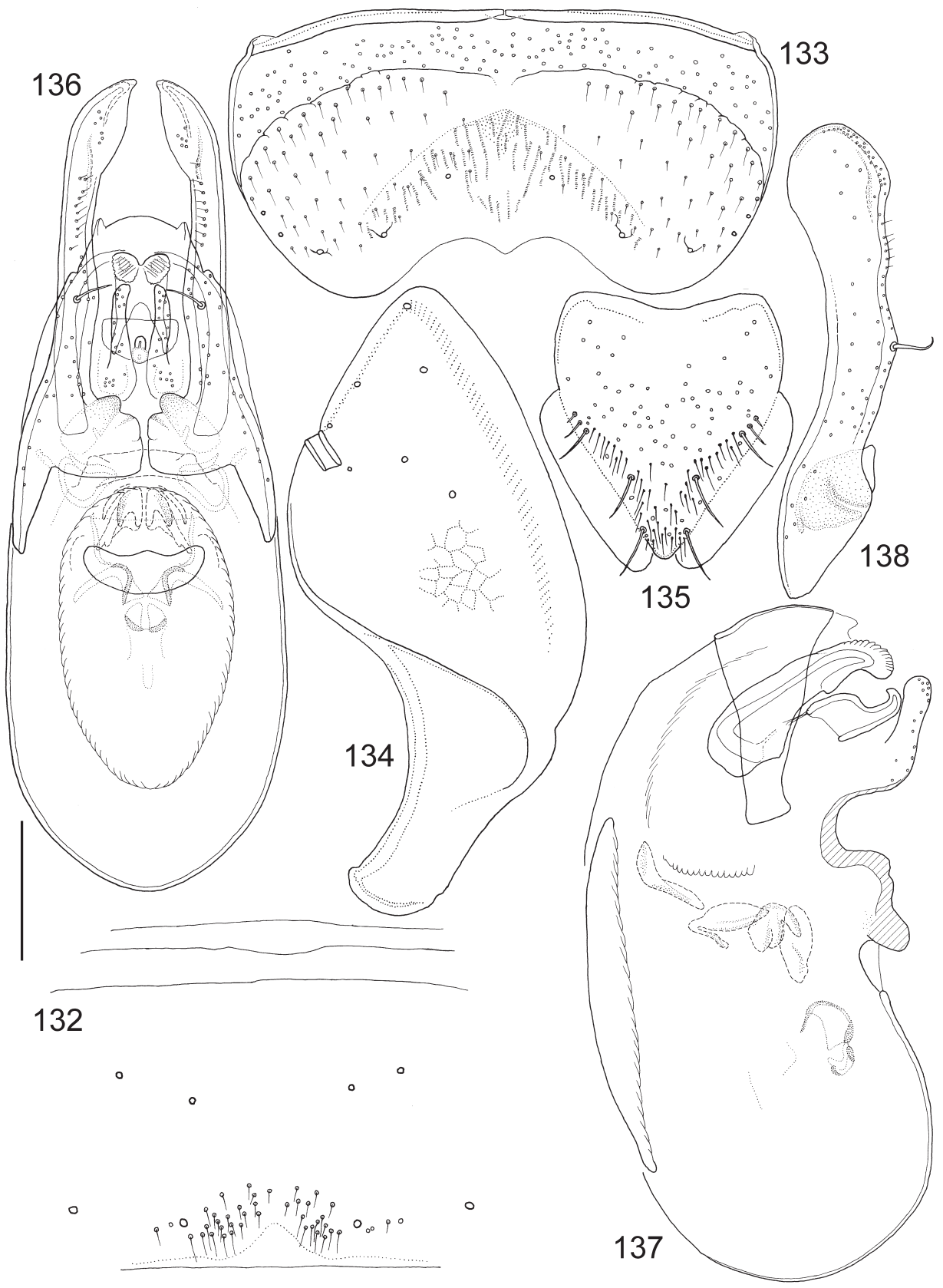

Figs 132-138. Anotylus hartmanni sp. n., male. 132 = median part of sternite VII, $133=$ sternite VIII, 134 = tergite IX, 135 = tergite X, 136 = aedeagus, frontal view, 137 = median lobe, lateral view, 138 = paramere, lateral view. Scales: $0.10 \mathrm{~mm}$ for Figs 136-138, $0.125 \mathrm{~mm}$ for Figs 134-135, $0.14 \mathrm{~mm}$ for Figs 132-133 
Head with anterior margin truncate in middle, oblique to supraantennal tubercles (as moderately elevated oblique ridges) where gently angled (broadly rounded) again, possessing slight rim, disc slightly impressed near eyes. Epistomal suture forming subcircular impression (less punctured inside), upper surface without tactile setae, eyes small and rather flat; in dorsal view temples (1.9× length of eye) alatiform, laterally bulging and rounded, posteriorly constricted to form distinct neck, however, dorsal side not separated by occipital furrow, rather by dorsum of neck bearing microsculpture instead of punctation. Antennomere 1 flattened club-shaped and smooth, segment 2 basally broad ovoid, segment 3 club-shaped, segment 4 small and isodiametrical, segments 5-11 with basal dishes, articles 6-10 slightly transverse (about $1.4 \times$ broader than long). Head and pronotum with mostly lacunose sculpture. Pronotum with lateral portion explanate and slightly up-turned, edge with a few short setae, lateral border rather evenly arched, with a number of small, irregularly distributed tubercles, posterior margin incised before corners; disc medially rather convex, with two posteriorly confluent longitudinal ridges, impressed alongside them, laterally impressed near side margins. Elytra with membranous posterior margin extending from sutural corner to lateral 5/6 of hind margin, a small incision-like thinning before outer corner. Elytral epipleural ridge present with up-turned lateral alatiform portions, disc depressed; shoulders moderately developed, surface foveolate, in between punctures torulose. Legs short, pro- and mesotibia with several spinulose rows, metatibia with longitudinal ctenidium of spinules in distal half. Abdomen with sides weakly arcuate, second segment with paratergites strongly broadening posteriorly (abdomen appearing constricted at base), those of segments III-VII thin, mesal paratergites broad. Posterior edge of tergite VII with thin palisade fringe, median part of sternite VII as in Fig. 132, sternite VIII (Fig. 133), tergites IX (Fig. 134), X (Fig. 135) and aedeagus as in Figs 136-138, spermatheca as in Fig. 267.

Etymology - The species is named after Matthias Hartmann (NKME), who contributed significantly to the better knowledge of the fauna of the Himalayas, particularly Nepal. Nepal.

Distribution - Only known from the Manaslu mountain range in Western

Remarks - Habitat notes from the collector (Joachim Schmidt) for the 40 paratypes collected in 2003: Forest remnants near temporary streams; species rich forests on both collecting sites, mainly evergreen trees and shrubs, but markedly influenced by tree cutting and cow/yak/sheep/goat grazing.

\section{Anotylus ijen sp. n. \\ (Figs 89, 139-145, 268)}

Type material - Holotype (ふ̋): INDONESIA/JAVA: E-, Banyuwangi, Gn. Ijen, Kluncing, $08^{\circ} 07.986^{\prime} \mathrm{S}, 1^{1} 4^{\circ} 11.834^{\prime} \mathrm{E}, 1130 \mathrm{~m}, 30 . \mathrm{VIII} .2006$, leg. A. Riedel, sifted (sample 2) (MBBJ); Paratypes (2): Banyuwangi, Gn. Ijen, Kluncing, 0808.070'S, 114¹1.810’E, 1110 m, 30.VIII.2006, leg. A. Riedel, sifted (sample 1), (1 $\delta^{\top}$, SMNK); Banyuwangi, Gn. Ijen, Licin, $08^{\circ} 07.115^{\prime} S$, $114^{\circ} 14.650^{\prime} \mathrm{E}, 1100$ m, 31.VIII.2006, leg. A. Riedel, sifted (sample 6), (1 +, SMNK).

Description - Measurements (in mm, n=3): HW $=0.61(0.60-0.62) ; \mathrm{TW}=0.63(0.61-$ 0.64); $\mathrm{PW}=0.91$ (0.90-0.92); $\mathrm{EW}=0.94$ (0.91-0.97); $\mathrm{AW}=0.98$ (0.94-1.03); $\mathrm{HL}=0.48$ (0.46- 


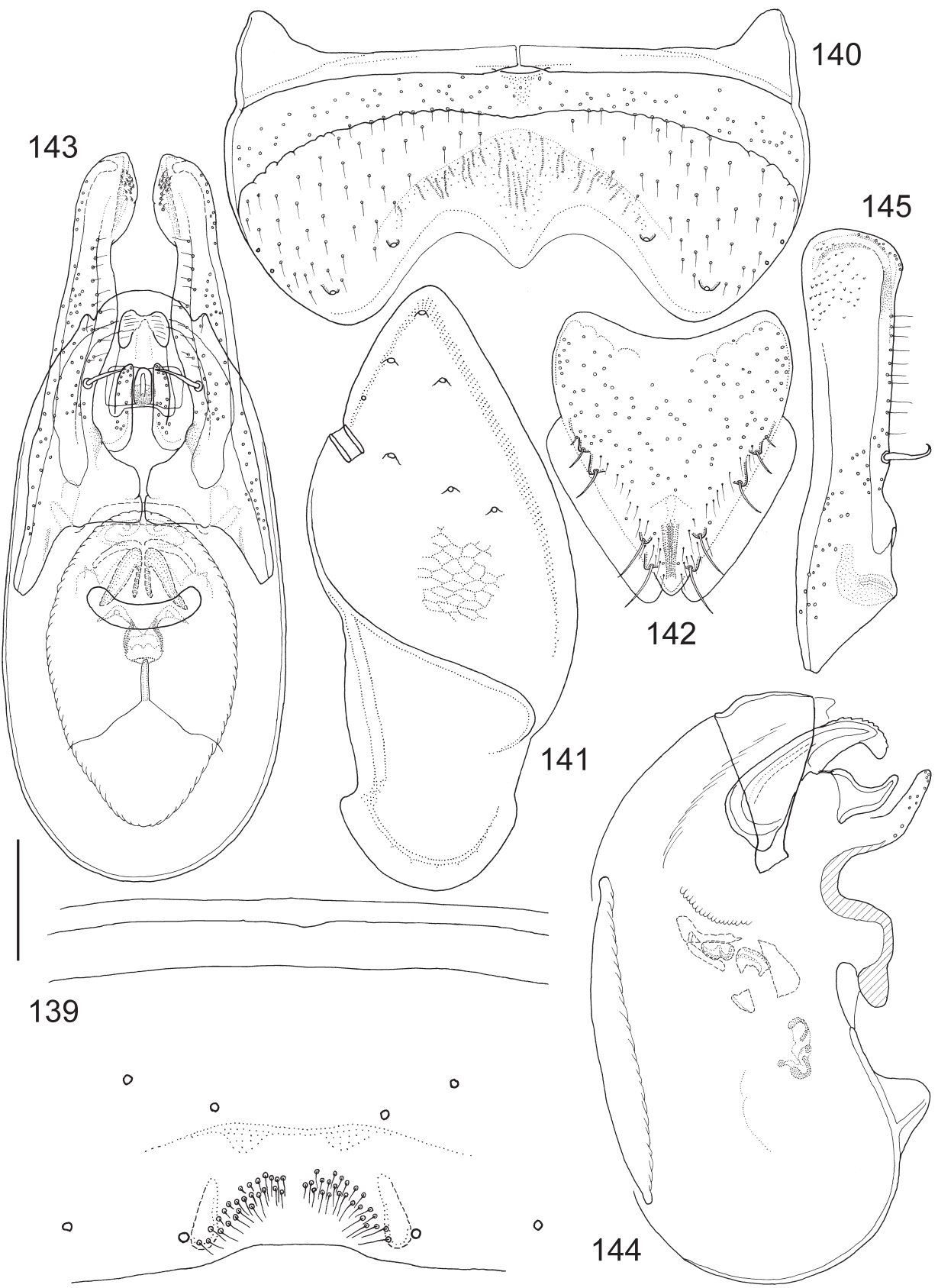

Figs 139-145. Anotylus ijen sp. n., male. $139=$ median part of sternite VII, $140=$ sternite VIII, 141 = tergite IX, 142 = tergite $X, 143$ = aedeagus, frontal view, 144 = median lobe, lateral view, 145 = paramere, lateral view. Scales: $0.10 \mathrm{~mm}$ for Figs 139, 142-145, $0.12 \mathrm{~mm}$ for Figs 140-141 
$0.50) ; \mathrm{EL}=0.12$ (0.11-0.12); $\mathrm{TL}=0.18$ (0.18-0.19); $\mathrm{PL}=0.51$ (0.50-0.52); $\mathrm{SC}=0.50$ (0.49-0.51); $\mathrm{FB}=1.54(1.52-1.56) ; \mathrm{BL}=3.00$ (2.91-3.05). Habitus as in Fig. 89. Forebody moderately shining despite sculpture, abdomen very finely microsculptured and medium finely, rather shallowly punctate, duller. Body reddish medium to dark brown, except explanate part of pronotum, clypeal area, mouthparts and antennae reddish medium brown. Head with anterior margin forming an arch truncate in middle, with very slight rim, supraantennal prominences as moderately elevated oblique ridges, disc slightly impressed near eyes, epistomal suture forming subcircular impression (almost impunctate inside) upper surface without tactile setae, eyes rather small and very slightly bulging from sideline of head; in dorsal view temples (1.5× length of eye) alatiform, laterally evenly rounded, posteriorly constricted to form distinct neck, however, dorsal side with only traces of occipital furrow, dorsum of neck demarcated by bearing microsculpture instead of punctation. Antennomere 1 club-shaped, smooth, segment 2 basally broad oval, segment 3 club-shaped, segment 4 small and isodiametrical, segments 5-11 with basal dishes, articles 6-10 transverse (more than $1.5 \times$ broader than long), antennomeres 9-10 bulkier than preceding. Head and pronotum with mostly foveate sculpture, with varying but mostly very small interspaces. Pronotum with lateral portion mostly explanate and up-turned, edge alatiform with sparse short setae; lateral margin unevenly arched with a number of small, irregularly distributed denticles, posterior margin strongly incised in a variable zigzagged fashion before corners; disc medially with two faint, posteriorly confluent longitudinal ridges, impressed alongside them, lateral parts impressed (mostly around middle). Elytra with membranous posterior margin extending from sutural corner to $4 / 5$ of hind margin, a small incision before outer corner. Elytral epipleural ridge present with up-turned lateral alatiform portions, disc longitudinally impressed alongside; shoulders well developed, surface foveolate, in between punctures torulose. Legs short, pro- and mesotibia with several spinulose rows, metatibia with longitudinal ctenidium of spinules in distal half. Abdomen with sides moderately arcuate, second segment with paratergites broadening posteriorly (abdomen appearing constricted at base), those of segments III-VII thin, mesal paratergites broad; posterior edge of tergite VII with thin palisade fringe, median part of sternite VII as in Fig. 139, sternite VIII (Fig. 140), tergites IX (Fig. 141), X (Fig. 142) and aedeagus as in Figs 143-145, spermatheca as in Fig. 268.

Etymology - The species is named after the mountain range Gunung Ijen, where it was found (noun in apposition).

Distribution - Only 3 specimens were found at 3 different but closely situated localities in Java.

Remarks - The localities (at Gn. Ijen) were remainders of primary forest (usually very wet). The low number of specimens probably do not correspond to rarity. The collector returned to the area one more time but could not collect because of the permanent heavy rains.

\section{Anotylus jaljulensis (Coiffait, 1984)}

(Figs 87, 146-152)

Oxytelopsis jaljulensis Coiffait, 1984: 148; Herman 2001: 1414. 
Examined type material - Oxytelopsis jaljulensis Coiffait - Holotype (†): “Nepal XI[19]78-3; Jaljale Himal; 2920 m [leg.] P C[assagnau]-3 \Oxytelopsis; jaljalensis; H. Coiffait det. $1983 \backslash$ Type [red card]" (MNHP).

Other material - NEPAL: Panchthar distr., Dhorpar Kharka $\left[27^{\circ} 04.5^{\prime} \mathrm{N}, 87^{\circ} 55.5^{\prime} \mathrm{E}\right]$, mature Rhododendron-Lithocarpus forest, 2700 m, 13-16.IV.[19]88, [leg.] J. Martens \& W. Schawaller (324) (1 $\delta^{\AA}$, MHNG); Taplejung distr., Omje Kharka NW Yamputhin $\left[27^{\circ} 27.4^{\prime} \mathrm{N}\right.$, 8753.2’E], mature mixed broad-leaved forest, 2300-2500 m, 1-6.V.[19]88, [leg.] J. Martens \& W. Schawaller (356) $(1 \stackrel{\jmath}{\circ}$, SMNS).

Redescription - Measurements (in mm, n=2): HW $=0.67(0.65-0.68) ; \mathrm{TW}=0.60(0.59$ $0.61) ; \mathrm{PW}=0.88(0.86-0.90) ; \mathrm{EW}=0.87(0.85-0.88) ; \mathrm{AW}=0.98(0.95-1.00) ; \mathrm{HL}=0.54(0.53-$ $0.54) ; \mathrm{EL}=0.11(0.11-0.11) ; \mathrm{TL}=0.22(0.21-0.22) ; \mathrm{PL}=0.60$ (0.59-0.60); $\mathrm{SC}=0.41(0.38-0.43)$; $\mathrm{FB}=1.64$ (1.59-1.68); BL = 3.51 (3.43-3.59). Habitus as in Fig. 87. Forebody moderately shining despite sculpture, abdomen with coriaceous microsculpture, moderately finely and shallowly punctured, less lustrous. Body reddish medium to dark brown except anterior margin of head, lateral margin of pronotum, antennomeres 1-4 and mouthparts medium brown, apex of last antennomere occasionally also lighter. Head with anterior margin truncate in middle, oblique to supraantennal tubercles (as moderately elevated oblique ridges) where gently angled again, possessing slight rim (obsolete at front), disc slightly impressed near eyes. Epistomal suture forming subcircular impression (almost unpunctured inside), upper surface without tactile setae, eyes small and extremely bulging (like half sphere); in dorsal view temples (twice the length of eye) alatiform, laterally unevenly rounded (slightly angled) and posteriorly constricted to form distinct neck, however, dorsal side not separated by occipital furrow, rather by dorsum of neck bearing microsculpture instead of punctation. Antennomere 1 flattened club-shaped and smooth, segment 2 basally broad ovoid, segment 3 apically incrassate, segment 4 almost round, segments 5-11 with basal dishes, articles 6-10 about as long as broad. Head and pronotum with mostly lacunose sculpture. Pronotum with lateral portion explanate and slightly up-turned, edge with a few short setae, lateral border unevenly arched with a number of small, irregularly distributed denticles, posterior margin slightly concave before corners; disc medially rather convex, with two faint, parallel longitudinal ridges, laterally impressed around middle of sides. Elytra with very thin membranous posterior margin extending from sutural corner to lateral $3 / 4$ of hind margin, a small incision-like thinning before outer corner. Elytral epipleural ridge present with up-turned lateral alatiform portions, disc depressed; shoulders moderately developed, surface foveolate, in between punctures torulose. Legs short, pro- and mesotibia with several spinulose rows, metatibia with longitudinal ctenidium of spinules in distal half. Abdomen with sides weakly arcuate, second segment with paratergites strongly broadening posteriorly (abdomen appearing constricted at base), those of segments III-VII thin, mesal paratergites broad. Posterior edge of tergite VII with thin palisade fringe, median part of sternite VII as in Fig. 146, sternite VIII (Fig. 147), tergites IX (Fig. 148), X (Fig. 149) and aedeagus as in Figs 150-152.

Distribution - The species is only known from a couple of localities in the easternmost part of Nepal.

Remarks - It appears that the name jaljulensis as printed in the original description is a result of misreading the locality: the label says Jaljala Himal and even on the author's handwritten name label the specific epithet is clearly 'jaljalensis'. But in order to emend the name, there must be evidence of a lap- 


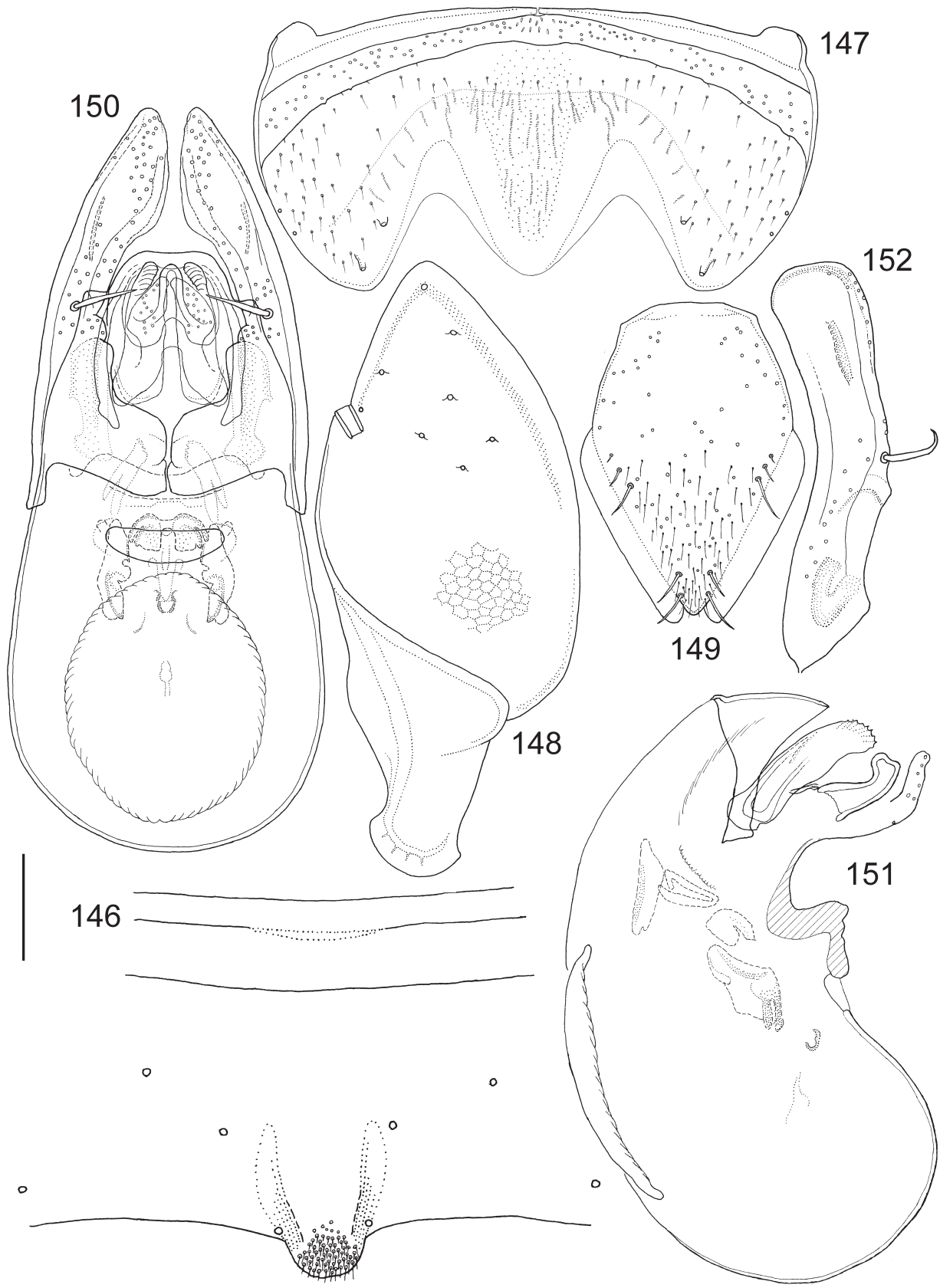

Figs 146-152. Anotylus jaljulensis (Coiffait, 1984), male. $146=$ median part of sternite VII, 147 = sternite VIII, 148 = tergite IX, $149=$ tergite $X, 150=$ aedeagus, frontal view, $151=$ median lobe, lateral view, 152 = paramere, lateral view. Scales: $0.10 \mathrm{~mm}$ for Figs 146, 150-152, $0.13 \mathrm{~mm}$ for Figs 148-149, $0.14 \mathrm{~mm}$ for Fig. 147 
sus in the original article, which is absent here. The locality of the holotype was published as "Jaljula Himal". Since there is only external evidence, the name is maintained.

\section{Anotylus jambi sp. n. \\ (Figs 88, 153-159, 269)}

Type material - Holotype (ð̋): INDONESIA/SUMATRA: Jambi, Mt. Kerinci, 2100 m, 14.XI.1989, leg. I. Löbl, D. Agosti, D. Burckhardt (16), sifting of vegetational debris, transition upper montane Lithocarpus-Castanopsis to moss forest (MHNG); Paratypes (7):

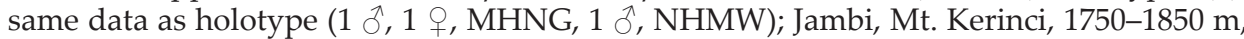
11-12.XI.1989, leg. I. Löbl, D. Agosti, D. Burckhardt (12a), sifting of vegetational debris (1 9, MHNG), Jambi prov., Mt. Kerinci, footpath to summit, W of Kersik Tua, $2160 \mathrm{~m}$ [01 $43^{\circ} 40^{\prime \prime} \mathrm{S}, 101^{\circ} 16^{\prime} 29^{\prime \prime} \mathrm{E}$ ], 17-18.II.2000, leg. P. Schwendinger (Sum-00/13), evergreen montane rainforest, sifting $(1 \hat{\jmath}, 1 \stackrel{1}{\circ}, \mathrm{MHNG}, 1 \hat{\jmath}$, SMNS).

Description - Measurements (in mm, n=8): HW = 0.87 (0.85-0.88); TW = 0.88 (0.86-0.90); $\mathrm{PW}=1.21$ (1.18-1.26); $\mathrm{EW}=1.13$ (1.09-1.16); $\mathrm{AW}=1.29$ (1.25-1.34); $\mathrm{HL}=0.61$ (0.58-0.63); $\mathrm{EL}$ $=0.15(0.14-0.16) ; \mathrm{TL}=0.25(0.24-0.26) ; \mathrm{PL}=0.75(0.72-0.78) ; \mathrm{SC}=0.60(0.58-0.63) ; \mathrm{FB}=2.00$ (1.94-2.05); $\mathrm{BL}=4.13$ (3.93-4.30). Habitus as in Fig. 88. Forebody moderately shining despite sculpture, abdomen very finely microsculptured (punctation totally obscured), rather dull. Whole body slightly reddish dark brown, clypeal area lighter (for transparency), first 4 antennomeres and mouthparts medium to light brown. Head with very thin rim on anterior margin, latter truncate in middle, otherwise very broadly rounded, supraantennal prominences as elevated oblique ridges, disc impressed near eyes. Epistomal suture forming circular impression (almost unpunctured inside), upper surface without tactile setae, eyes rather small and very slightly bulging from sideline of head; in dorsal view temples (1.6× length of eye) alatiform, laterally rather bulging (and setose), posteriorly constricted to form distinct neck, however, dorsal side with only traces of occipital furrow, dorsum of neck demarcated by bearing microsculpture instead of punctation. Antennomere 1 clubshaped, smooth, segment 2 basally broad oval, segment 3 club-shaped, segment 4 small and isodiametrical (with rudimentary basal dish), segments 5-11 with basal dishes, articles 6-10 transverse (about $1.5 \times$ broader than long), segment 11 somewhat lighter at apex. Head and pronotum with mostly lacunose sculpture. Pronotum with lateral portion mostly explanate and slightly up-turned, edge uneven with sparse short setae, anterior corners somewhat tuberculate (edge inward turned) and distinctly setose, lateral margin more or less arcuate, posterior margin before corners shallowly and broadly incised; disc medially with two parallel longitudinal ridges, slightly impressed alongside them, laterally strongly impressed around middle of sides (as a large pit). Elytra with membranous posterior margin extending from sutural corner to 5/6 of hind margin. Elytral epipleural ridge apparent as stronger sculpture lines in lateral alatiform portions, disc somewhat impressed along midline of each elytron; shoulders well developed, surface foveolate, in between punctures torulose. Legs short, pro- and mesotibia with several spinulose rows, metatibia with longitudinal ctenidium of spinules in distal half. Abdomen with sides moderately arcuate, second segment with paratergites broadening posteriorly (abdomen appearing constricted at base), those of segments III-VII thin, mesal paratergites broad; posterior edge of tergite VII with thin palisade fringe, median part of sternite VII as in Fig. 153, sternite VIII (Fig. 154), tergites IX (Fig. 155), X (Fig. 156) and aedeagus as in Figs 157-159, spermatheca as in Fig. 269. 


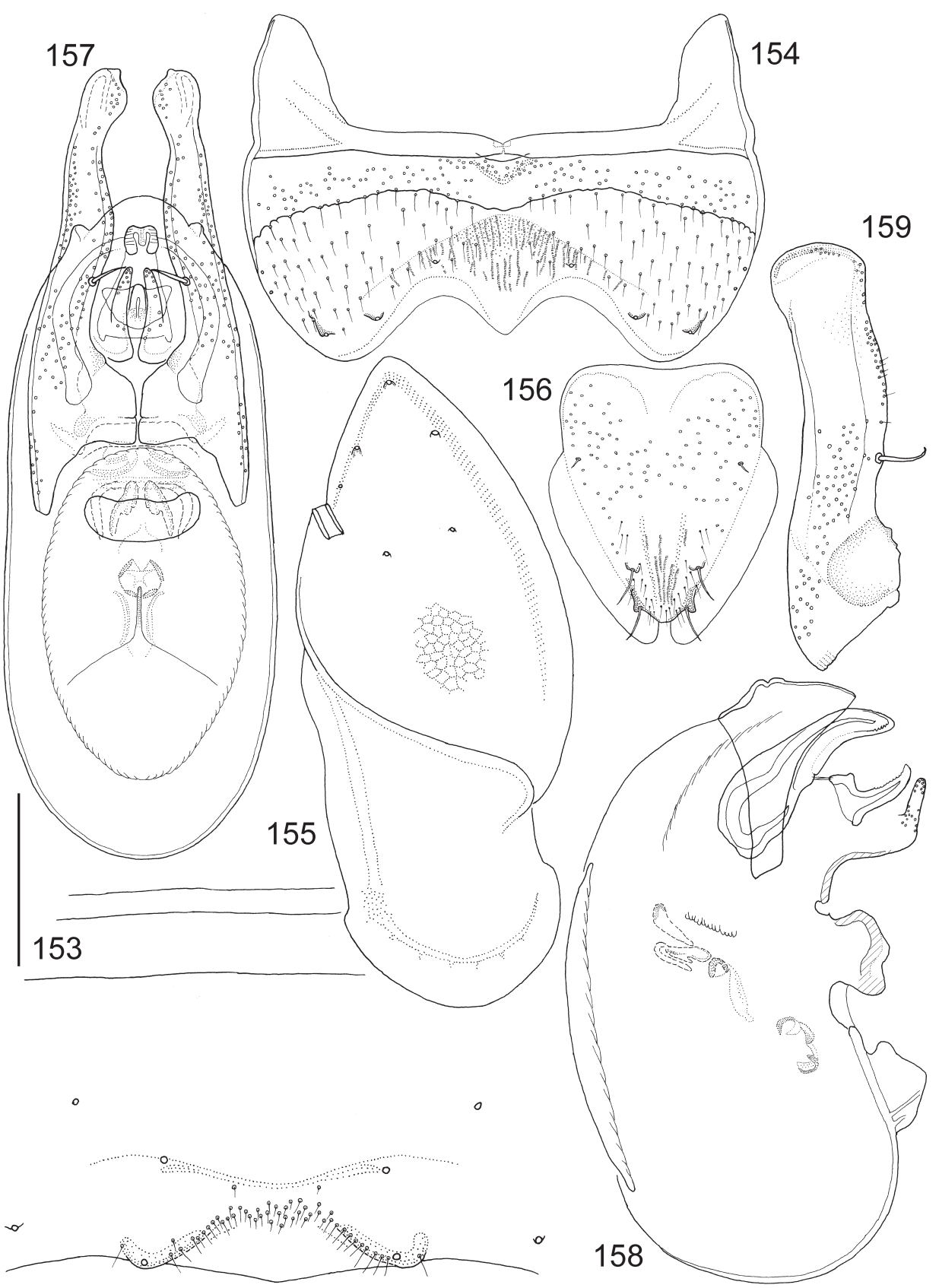

Figs 153-159. Anotylus jambi sp. n., male. $153=$ median part of sternite VII, $154=$ sternite VIII, $155=$ tergite IX, 156 = tergite $X, 157=$ aedeagus, frontal view, $158=$ median lobe, lateral view, 159 = paramere, lateral view. Scales: $0.20 \mathrm{~mm}$ for Figs 153, 157-159, $0.24 \mathrm{~mm}$ for Fig. 156, $0.26 \mathrm{~mm}$ for Fig. 155, $0.29 \mathrm{~mm}$ for Fig. 154 
Etymology - The species is named after the administrative unit (Jambi) of the type locality (noun in apposition).

Distribution - The species appears to be a high altitude endemic, has only been found at two closely situated localities in Sumatra (Indonesia). The type locality may have been the very same site where P. Schwendinger collected; the approximate coordinates were determined with his help.

Remarks - According to the only record with specific information it was sifted from vegetable debris near the transition zone from upper montane Lithocarpus-Castanopsis to moss forest.

\section{Anotylus kabasi sp. n. (Figs 98, 160-166, 270)}

Type material - Holotype (§): INDONESIA/SUMATRA: North Sumatra, Medan Kabanjahe, Gn. Sinabung [0310‘00“N, 98²3‘50“E], 2000 m, 7-8.X.1990, leg. A. Riedel (SMNS); Paratypes (236): same data as holotype (8 $01,9+9$, SMNS); North Sumatra, Brastagi, Gunung Sibayak, 20.II.1990, leg. H. Schillhammer (9, NHMW), same but 1300 m, 6-19.VIII.1992, leg. Barries \& Cate (4, NHMW); North Sumatra, 7 km N Brastagi, 1500 m, 2.XII.1989, leg. I. Löbl, D. Agosti, D. Burckhardt (28a), sifting of vegetational debris in degraded montane

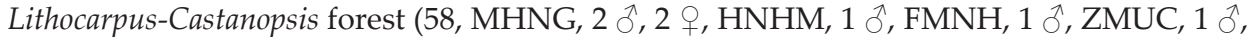

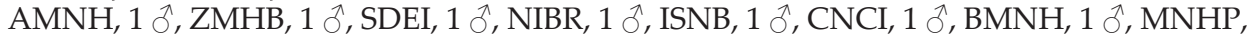
1 है, NMPC, 1 है, MZLU); North Sumatra, 5 km W Brastagi, Tongkoh, 1450 m, 3.XII.1989, leg. I. Löbl, D. Agosti, D. Burckhardt (29a), mixed pine forest with many epiphytes, sifting of vegetational debris (42, MHNG); West Sumatra, Bukittinggi, Gn. Merapi [00²2`S, 100²7`E], 1900-2100 m, 18.X.1990, leg. A. Riedel (2 §’, 2 ㅇ, SMNS); Jambi, Mt. Kerinci, 1750-1850 m, 11-12.XI.1989, leg. I. Löbl, D. Agosti, D. Burckhardt (11), sifting of vegetational debris in montane Lithocarpus-Castanopsis forest (1 $\widehat{\delta}, 49, \mathrm{MHNG})$; Jambi, Mt. Kerinci, 1900 m, 13.XI.1989, leg. I. Löbl, D. Agosti, D. Burckhardt (15a), sifting of vegetational debris in montane Lithocarpus-Castanopsis forest (1, MHNG); Jambi, W Mt. Tujuh Lake, 1400 m, 14.XI.1989, leg. I. Löbl, D. Agosti, D. Burckhardt (17), sifting of vegetational debris in montane Lithocarpus-Castanopsis forest (32, MHNG, 1 §े, HNHM); East Sumatra, Lampung, Kalianda, Gunung Rajabasa, $05^{\circ} 46.656^{\prime} \mathrm{N}, 105^{\circ} 37.478^{\prime} \mathrm{E}, 1174 \mathrm{~m}, 15 . \mathrm{VIII} .2006$, leg. A. Riedel, sifted (sample 2) (SMNK); INDONESIA/JAVA: West Java, Cibodas Botanical Garden, near Cipanas, ca. 50 km E Bogor, 1400 m, 3-6.XI.1989, leg. I. Löbl, D. Agosti, D. Burckhardt (2a), sifting of vegetational debris in montane Lithocarpus-Castanopsis forest (1, MHNG).

Description - Measurements (in mm, $\mathrm{n}=10): \mathrm{HW}=0.77(0.71-0.80) ; \mathrm{TW}=0.77(0.70-0.80)$; $\mathrm{PW}=1.14$ (1.04-1.17); EW = 1.20 (1.09-1.30); $\mathrm{AW}=1.16$ (1.07-1.25); HL = 0.57 (0.52-0.61); EL $=0.15(0.13-0.16) ; \mathrm{TL}=0.24(0.22-0.25) ; \mathrm{PL}=0.66(0.58-0.71) ; \mathrm{SC}=0.77(0.62-0.85) ; \mathrm{FB}=$ 2.06 (1.77-2.21); BL = 3.65 (3.35-3.95). Habitus as in Fig. 98. Forebody moderately shining despite sculpture, abdomen very finely microsculptured and medium finely, rather shallowly punctate, duller. Forebody blackish dark brown with reddish tint (except explanate lateral part of pronotum and clypeal area lighter, reddish), abdomen and legs reddish dark brown, mouthparts and antennae reddish medium brown. Head with a thin rim on anterior margin, truncate in middle, very slightly angled also at supraantennal prominences, these as moderately elevated oblique ridges, disc slightly impressed near eyes. Epistomal suture 


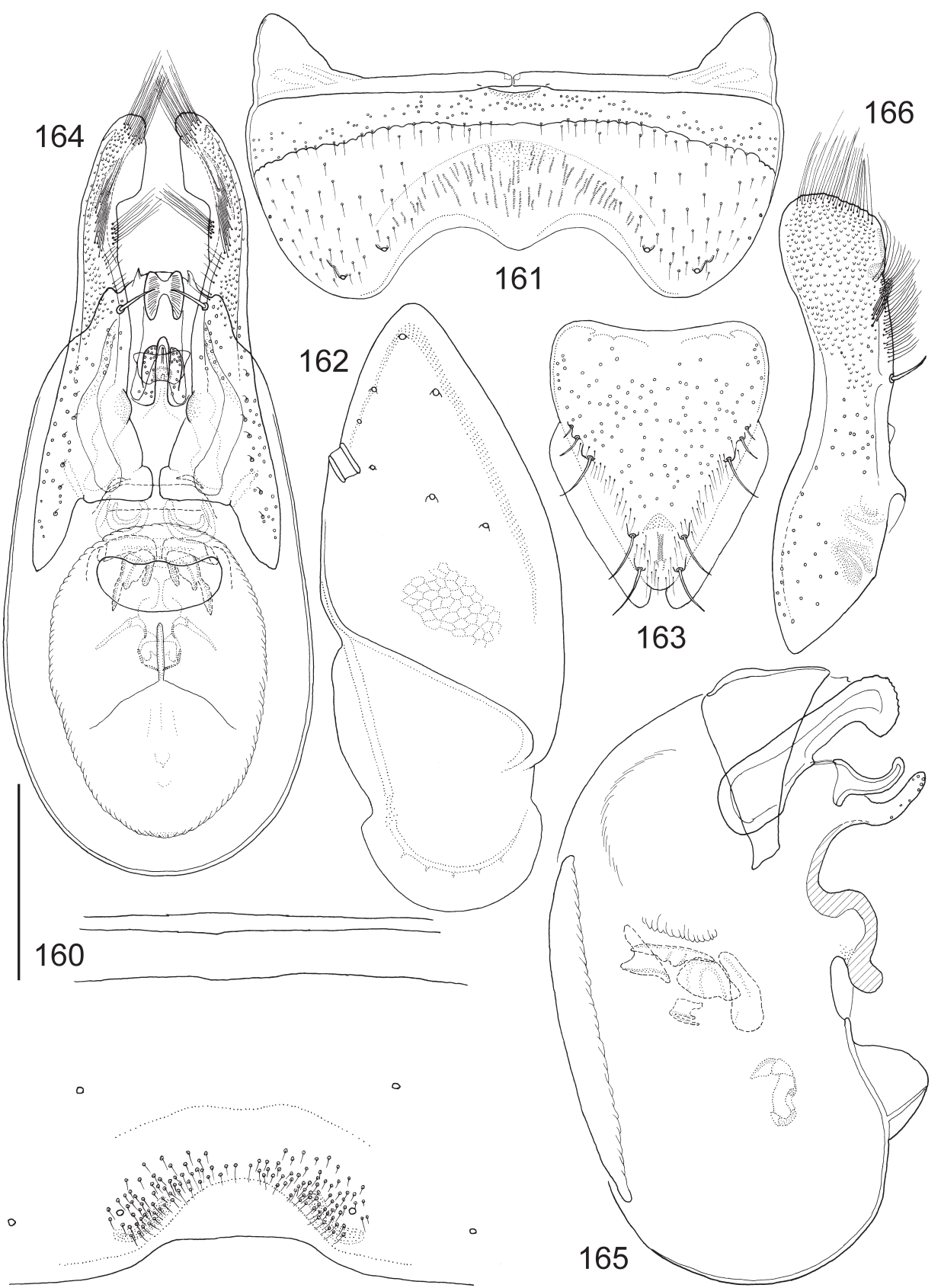

Figs 160-166. Anotylus kabasi sp. n., male. $160=$ median part of sternite VII, $161=$ sternite VIII, 162 = tergite IX, 163 = tergite X, 164 = aedeagus, frontal view, $165=$ median lobe, lateral view, 166 = paramere, lateral view. Scales: $0.20 \mathrm{~mm}$ for Figs 160, 164-166, $0.23 \mathrm{~mm}$ for Fig. 163, $0.27 \mathrm{~mm}$ for Fig. 162, $0.30 \mathrm{~mm}$ for Fig. 161 
forming subcircular impression (unpunctured inside), upper surface without tactile setae, eyes medium sized, moderately bulging from sideline of head; in dorsal view temples (1.6× length of eye) alatiform, laterally evenly rounded and posteriorly constricted to form distinct neck, not separated dorsally by occipital furrow, only by neck being microsculptured rather than punctured. Antennomere 1 club-shaped, finely sculptured, segment 2 elongate oval, segment 3 club-shaped, segment 4 small and isodiametrical, segments $5-11$ with basal dishes, articles 6-10 transverse (about $1.3 \times$ broader than long). Head and pronotum with foveate sculpture. Pronotum with lateral portion explanate and slightly up-turned, edge alatiform with sparse short setae; lateral margin more or less evenly arched with a number of small, irregularly distributed denticles, posterior margin weakly incised before corners; disc medially with two parallel longitudinal ridges (almost confluent posteriorly), impressed alongside them, laterally impressed around middle of sides. Elytra with membranous posterior margin extending from sutural corner to 3/4 of hind margin. Elytral epipleural ridge present with up-turned lateral alatiform portions, disc longitudinally impressed alongside; shoulders well developed, surface lacunose, small interspaces torulose. Legs short, pro- and mesotibia with several spinulose rows, metatibia with longitudinal ctenidium of spinules in distal half. Abdomen with sides rather arcuate, second segment with paratergites strongly broadening posteriorly (abdomen appearing constricted at base), those of segments III-VII thin, mesal paratergites broad; posterior edge of tergite VII with thin palisade fringe, median part of sternite VII as in Fig. 160, sternite VIII (Fig. 161), tergites IX (Fig. 162), X (Fig. 163) and aedeagus as in Figs 164-166, spermatheca as in Fig. 270.

Etymology - The specific epithet is combined from the first letters of the words 'Kabanjahe' and 'Sinabung' of the type locality (noun in apposition).

Distribution - The species is known from several localities across Sumatra and a single female specimen was found in W Java.

Remarks - It is one of those species for which a brachypter morph is known, the paratype from Gn. Rajabasa and part of those from Gn. Sinabung belong to it.

\section{Anotylus linaxi sp. n. (Figs 77, 167-173, 271)}

Type material - Holotype (ð): CHINA: N-Yunnan, Lijiang Naxi Aut. Co., 3 km NW

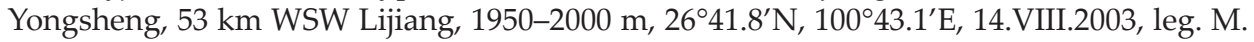
Schülke (C03-03), SE slope, secondary broadleaved forest (coll. Schülke in ZMHB); Paratypes (25): same data as holotype (1 0 , , 8, coll. Schülke, 1, AMNH, 1, MNHP, 1, ISNB, 1 ,

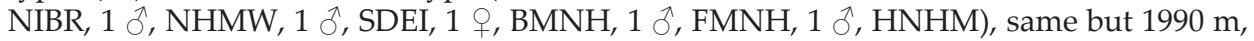
leg. A. Smetana (C122), sifting of layers of leaf litter and other debris under tall bushes open area (3, coll. Smetana/NSMT, 1 CNCI, $1 \hat{\delta}, \mathrm{ZMUC}, 1 \hat{\jmath}$, NMPC); THAILAND: Chiang Mai, Doi Inthanon, ravin très humide, 1650 m, 7.XI.1985, leg. D. Burckhardt \& I. Löbl (16), tamisage de débris végétaux et de champignons près d'un ruisselet ( 1 +, MHNG).

Description - Measurements (in mm, $\mathrm{n}=10)$ : $\mathrm{HW}=0.59(0.57-0.62)$; $\mathrm{TW}=0.58(0.55-$ $0.62) ; \mathrm{PW}=0.77(0.73-0.80) ; \mathrm{EW}=0.86(0.81-0.90) ; \mathrm{AW}=0.87(0.82-0.93) ; \mathrm{HL}=0.47(0.44-$ $0.50) ; \mathrm{EL}=0.13(0.13-0.15) ; \mathrm{TL}=0.19(0.19-0.20) ; \mathrm{PL}=0.50(0.49-0.54) ; \mathrm{SC}=0.58(0.56-0.61)$; $\mathrm{FB}=1.65$ (1.61-1.77); BL $=3.23$ (2.85-3.55). Habitus as in Fig. 77. Forebody weakly shining 


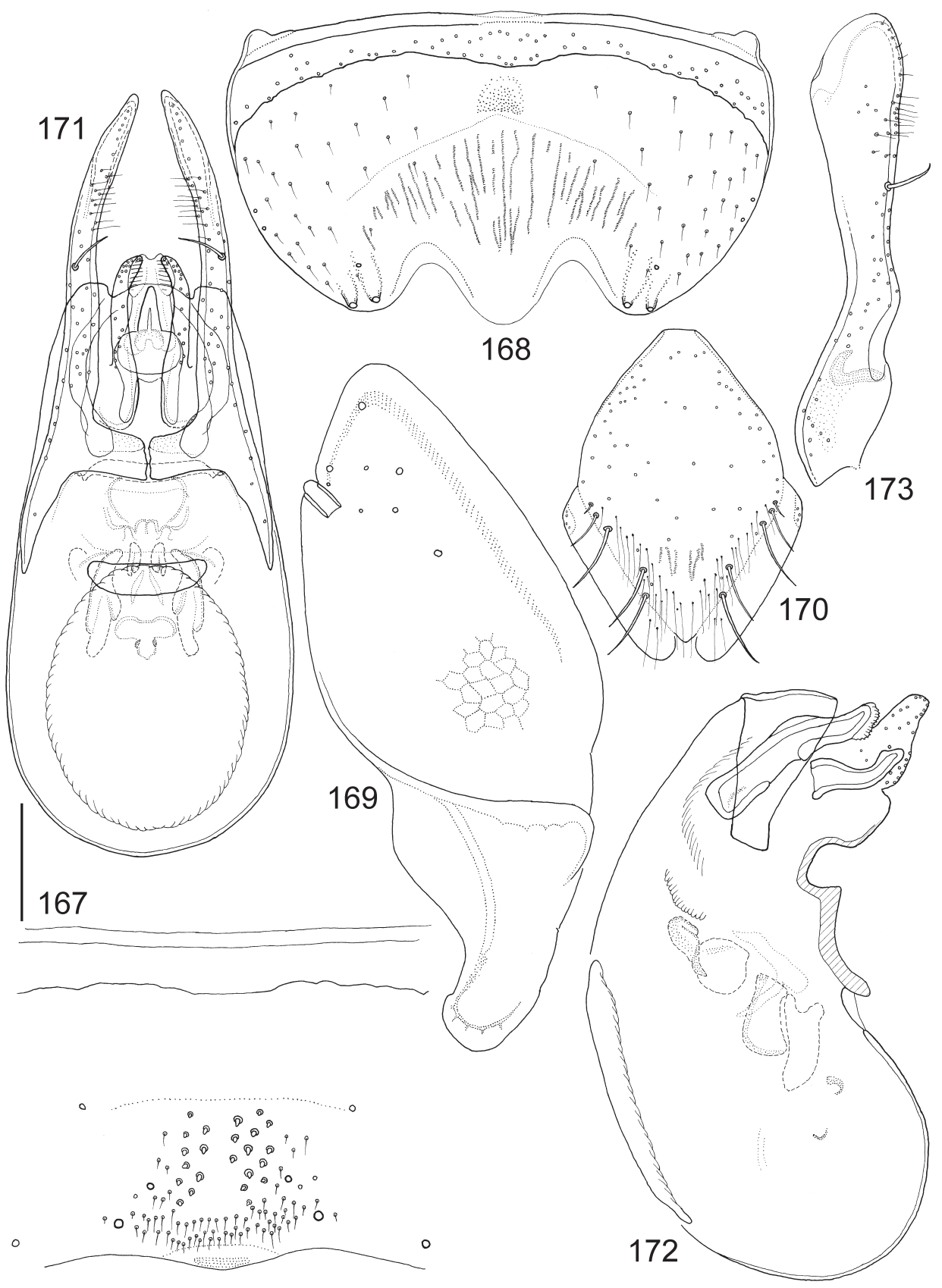

Figs 167-173. Anotylus linaxi sp. n., male. $167=$ median part of sternite VII, $168=$ sternite VIII, $169=$ tergite IX, 170 = tergite X, 171 = aedeagus, frontal view, $172=$ median lobe, lateral view, 173 = paramere, lateral view. Scales: $0.09 \mathrm{~mm}$ for Figs 170, $0.10 \mathrm{~mm}$ for Figs 171-173, $0.11 \mathrm{~mm}$ for Figs 167, 169, $0.12 \mathrm{~mm}$ for Fig. 168 
because of sculpture, abdomen with larger but indistinctly bordered and shallow punctures, traces of microsculpture, somewhat more lustrous. Body reddish medium brown, head (except clypeal area) often darker, dark brown, middle of tergite bases often blackish as well as most of antennomere 1 . Head with anterior margin possessing rim (weaker in middle), evenly arched but slightly angled at supraantennal prominences, these as moderately elevated oblique ridges, rim continuing posteriad along inner border of eyes, disc slightly impressed beside it. Epistomal suture forming circular impression (less sculptured inside), upper surface with weak tactile setae (intratemporalis) situated postero-medially of each eye. Eyes medium-sized and slightly bulging, in dorsal view temples $(1.4 \times$ length of eye) alatiform, laterally rounded and posteriorly constricted to form distinct neck, separated by transversal, weak occipital furrow. Antennomere 1 flattened club-shaped and strongly microsculptured, segment 2 oval but broad at base, segment 3 club-shaped, segment 4 small and slightly transverse, segments 5-11 with basal dishes, articles 6-10 transverse (about $1.5 \times$ broader than long), apex of segment 11 lighter. Head with areolaterugose, pronotum with lacunose sculpture. Pronotum with lateral portion explanate and slightly up-turned, edge alatiform with very sparse short setae; lateral border consisting of two longer straight sections at an angle connected by short concave section with thin marginal ridge and a number of small, indistinct denticles, posterior margin evenly arched; disc medially with two nearly parallel and posteriorly confluent longitudinal ridges, posteriorly impressed alongside them, laterally somewhat impressed towards side margin. Elytra with thin membranous posterior margin extending from sutural corner to 3/4 of hind margin. Elytral epipleural ridge apparent as stronger sculpture lines in lateral alatiform portions, disc with very slight to no impression in centre; shoulders well developed, surface obscurely foveolate and in between punctures torulose. Legs short, pro- and mesotibia with several spinulose rows, metatibia with longitudinal ctenidium of spinules in distal half. Abdomen with sides moderately arcuate, second segment with paratergites broadening posteriorly (abdomen slightly constricted at base), those of segments III-VII thin, mesal paratergites broad; posterior edge of tergite VII with thin palisade fringe, median part of sternite VII as in Fig. 167, sternite VIII (Fig. 168), tergites IX (Fig. 169), X (Fig. 170) and aedeagus as in Figs 171-173, spermatheca as in Fig. 271.

Etymology - The specific epithet is a combination of the first letters of 'Lijiang' and 'Naxi', from the locality label (noun in apposition).

Distribution - The know distribution is limited to Yunnan (China) and Chiang Mai (Thailand).

Remarks - The species is remarkably similar to A. reitteri and A. chinensis, differs only in its smaller size, less sculptured body surface and a slight difference in pronotal shape (less rounded sides with a characteristic "step" before the middle).

\section{Anotylus lucidulus (Cameron, 1936) (Figs 90, 174-180)}

Oxytelopsis lucidula Cameron, 1936: 30; Herman 1970: 413; Herman 2001: 1414. 
Examined type material - Oxytelopsis lucidula Cameron - Lectotype ( $\partial$, here designated): "Lecto-; type [lilac margined disc, curator label] \ Type [red margined disc] \F.C. Drescher; G. Tangkoeban Prahoe; 4000-5000 Voet[feet]; Preanger. Java; 12-18.I.1933 \Java: [yellow line under]; F.C. Drescher; B.M. 1934-264. \Oxytelopsis; lucidula; Type [purple, underlined] Cam \Oxytelopsis; lucidula Cam.; P.M. Hammond; det. 1970; Lectotype" (BMNH).

Other material - INDONESIA/JAVA: W-, 15 km N Bandung, Situ Lembang, ca. 1500 m, 7.VIII.1994, leg. R. Schuh (2 §, NHMW), Volcan Tangkuban Prahu, env. 30 km N Bandung, au pied du volcan, 7.VIII.1984, leg. J. Robert (29), extraction par appareil Winkler-

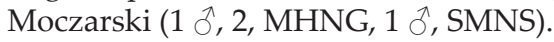

Redescription - Measurements (in $\mathrm{mm}, \mathrm{n}=7)$ : $\mathrm{HW}=0.87(0.84-0.92) ; \mathrm{TW}=0.87(0.83-$ 1.92); $\mathrm{PW}=1.29$ (1.25-1.38); $\mathrm{EW}=1.33$ (1.30-1.39); $\mathrm{AW}=1.38$ (1.30-1.44); $\mathrm{HL}=0.61$ (0.59$0.64) ; \mathrm{EL}=0.15(0.15-0.16) ; \mathrm{TL}=0.25(0.24-0.27) ; \mathrm{PL}=0.74(0.71-0.80) ; \mathrm{SC}=0.62(0.60-0.65)$; $\mathrm{FB}=2.03(1.95-2.15) ; \mathrm{BL}=4.07$ (3.86-4.31). Habitus as in Fig. 90. Forebody rather shining despite sculpture, abdomen very finely microsculptured and medium finely, rather shallowly punctate, duller. Body reddish dark brown, head sometimes darker, blackish, clypeal area and explanate part of pronotum lighter, more reddish, mouthparts and antennomeres 1-4 reddish medium brown, rest of antennomeres reddish medium to dark brown. Head with anterior margin forming an arch truncate in middle, with very slight rim, supraantennal prominences as moderately elevated oblique ridges, disc slightly impressed near eyes, epistomal suture forming subcircular impression (finer punctation inside, but just as dense) upper surface without tactile setae, eyes rather small and very slightly bulging from sideline of head; in dorsal view temples (1.6× length of eye) alatiform, laterally evenly rounded, posteriorly constricted to form distinct neck, however, dorsal side with only traces of occipital furrow, dorsum of neck demarcated by bearing microsculpture instead of punctation. Antennomere 1 club-shaped, smooth, segment 2 basally broad oval, segment 3 club-shaped, segment 4 small and isodiametrical, segments 5-11 with basal dishes, articles 6-10 transverse (about 1.5× broader than long), antennomeres 9-10 bulkier than preceding. Head and pronotum with mostly foveate sculpture, varying but mostly very small interspaces. Pronotum with lateral portion mostly explanate and up-turned, edge alatiform with sparse short setae; lateral margin more or less evenly arched with a number of small, irregularly distributed denticles, posterior margin strongly incised in a variable zigzagged fashion before corners; disc medially with two faint, posteriorly confluent longitudinal ridges, impressed alongside them, lateral parts impressed (mostly around middle). Elytra with conspicuous membranous posterior margin extending from sutural corner to $4 / 5$ of hind margin, a small incision before outer corner. Elytral epipleural ridge present with up-turned lateral alatiform portions, disc longitudinally impressed alongside; shoulders well developed, surface foveolate, in between punctures torulose. Legs short, pro- and mesotibia with several spinulose rows, metatibia with longitudinal ctenidium of spinules in distal half. Abdomen with sides moderately arcuate, second segment with paratergites broadening posteriorly (abdomen appearing constricted at base), those of segments III-VII thin, mesal paratergites broad; posterior edge of tergite VII with thin palisade fringe, median part of sternite VII as in Fig. 174, sternite VIII (Fig. 175), tergites IX (Fig. 176), X (Fig. 177) and aedeagus as in Figs 178-180.

Distribution - The species is apparently only known from a small area in Java (Indonesia). 


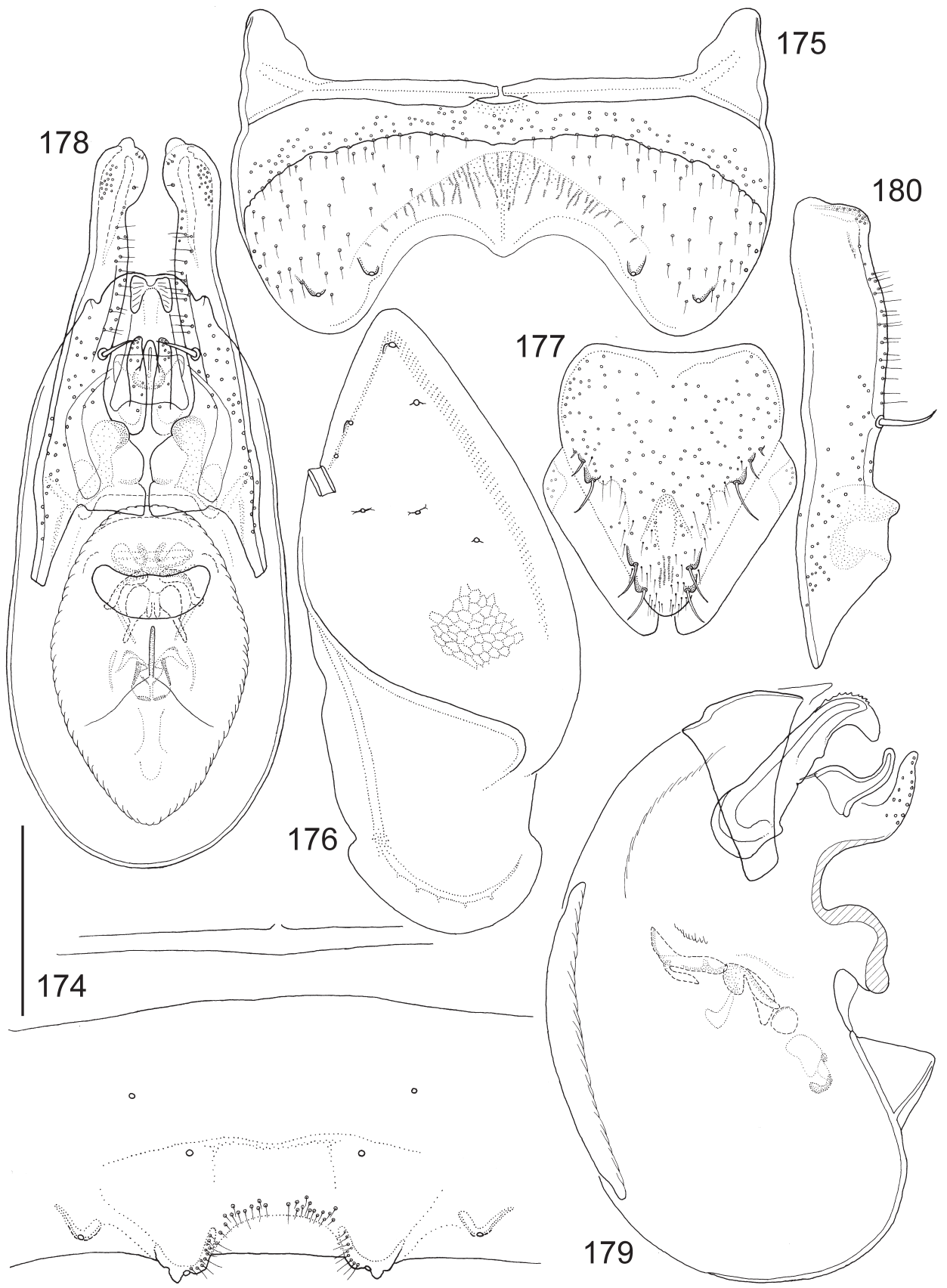

Figs 174-180. Anotylus lucidulus (Cameron, 1936), male. 174 = median part of sternite VII, 175 = sternite VIII, 176 = tergite IX, 177 = tergite $X, 178$ = aedeagus, frontal view, $179=$ median lobe, lateral view, 180 = paramere, lateral view. Scales: $0.20 \mathrm{~mm}$ for Figs 178-180, 0.23 $\mathrm{mm}$ for Figs 174, 177, 0.27 mm for Figs 175-176 
Remarks - Although Hammond (1975: 172) writes “O. lucidula is known from only one specimen" and thereby seems to validate his lectotype designation, his statement is clearly mistaken, as another syntype is hinted in the original description (by collecting date ranges) and listed as deposited in MBBJ (Ubaidillah 2002). Since this situation is debatable, the lectotype designation is here validated.

\section{Anotylus malaisei (Scheerpeltz, 1965)}

(Figs 95, 181-187)

Oxytelopsis malaisei Scheerpeltz, 1965: 152; Herman 1970: 413; Herman 2001: 1414.

Oxytelopsis gardneri Paulian, 1940: 58 (preoccupied); Paulian 1941: 161; Herman 1970: 413;

HERMAN 2001: 1414.

Examined type material - Oxytelopsis malaisei Scheerpeltz - Holotype ( $($ ): “N. E. Burma; Kambaiti 7000ft; 4-8/6[VI] 1934; [leg.] R. Malaise \ Holotypus \Typus; Oxytelopsis; Malaisei; O. Scheerpeltz \Oxytelopsis; Malaisei; nov. spec.; det. Scheerpeltz, $1940 \backslash 8012 ;$ E91 \ NHRS-VKBS; 000000132 \Anotylus; malaisei (Scheerpeltz); det. Makranczy, 2013" (NHRS).

Other material - NEPAL: E-, Kosi, Forêt S Mangsingma, 2200 m, ravin, 11.IV.1984, leg. I. Löbl \& A. Smetana (13), tamisage de feuilles mortes, mousses et bois pourri (34, MHNG, 1 §̊, HNHM); E-, Kosi, Val. Induwa Khola, 2000 m, 16.IV.1984, leg. I. Löbl \& A. Smetana (24a), tamisage mousses et feuilles mortes sur un terrain marécageux (15, MHNG, 2 ๙ , 1 ㅇ, HNHM); E-, Kosi, Val. Induwa Khola, 2000 m, 16.IV.1984, leg. I. Löbl \& A. Smetana (24b), tamisage mousses, branches et feuilles mortes, assez sec (8, MHNG); E-, Kosi, Val. Induwa Khola, 2100 m, lisière de la forêt, 17.IV.1984, leg. I. Löbl \& A. Smetana (26), tamisage de bois pourri et de feuilles mortes (8, MHNG); E-, Kosi, Val. Induwa Khola, 2100 m, 17.IV.1984, leg. I. Löbl \& A. Smetana (27), tamisage mousses et feuilles mortes au pied d'une falaise (17, MHNG); E-, Kosi, Val. Induwa Khola, 2000 m, 18.IV.1984, leg. I. Löbl \& A. Smetana (29), tamisage de débris végétaux accumulés au bord de la rivière (1, MHNG); E-, Kosi, Val. Induwa Khola, 2000-2600 m, 16-18.IV.1984, leg. I. Löbl \& A. Smetana (30), sous les pierres, sur la végétation (3, MHNG). E-, Kosi, 2 km E Mangsingma, 1900 m, 19.IV.1984, leg. I. Löbl \& A. Smetana (31a), tamisage de mousses très humides (2, MHNG); E-, Kosi, Forêt NE Kuwapani, 2250 m, 24.IV.1984, leg. I. Löbl \& A. Smetana (37), tamisage de branches pourries et mousses au-dessus d'un arbre couché (3, MHNG); distr. Kathmandu, Phulcoki, 2500 m, face nord, 28-29.IV.1984, leg. I. Löbl \& A. Smetana (41), tamisage de feuilles mortes et branches pourries au pied des roches (1, MHNG); INDIA: Meghalaya, Khasi Hills, audessus de Shillong, N-slope, 1850-1950 m, forêt primaire du Shillong Peak, 25.X.1978, leg. C. Besuchet \& I. Löbl (27), tamisage en forêt (4, MHNG, 1, HNHM), same but 30.X.1978 (35b) (1, MHNG); Meghalaya, East Khasi Hills distr., Mawphlang, Lawkyntang (sacred

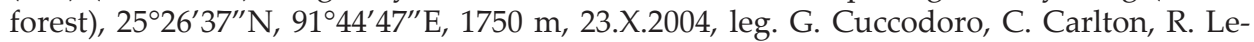
schen \& D. Errne (11b), sifting leaf litter with fungi on flat area near stream (1, MHNG); Assam, North Cachar Hills distr., Mt. Borail, Borail Peak, $25^{\circ} 06^{\prime} 43^{\prime \prime} \mathrm{N}, 93^{\circ} 03^{\prime} 11^{\prime \prime} \mathrm{E}, 1700$ m, 19.X.2005, leg. G. Cuccodoro \& A. Marletta (8a), sifting bamboo leaf litter in mountain rainforest (1, MHNG); CHINA: Yunnan, Dali Bai Auton. Prefecture, Jizu Shan, path to ca-

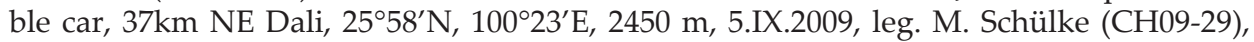
mixed forest, sifted from litter, moss \& pine apples $(1 \hat{\delta}, 6$, coll. Schülke, $1 \hat{\jmath}, \mathrm{HNHM}, 1$ §., NHMW, 1, AMNH, 1, FMNH, 1, NIBR, 1, ISNB, 1, ZMUC), same but leg. D.W. Wrase (29), mixed forest, litter, moss sifted (1, coll. Schülke); Yunnan, Lincang Pref., Laobie Shan, 
Wei Bo Shan pass, $24^{\circ} 08^{\prime} 16^{\prime \prime} \mathrm{N}, 9^{\circ} 42^{\prime} 53^{\prime \prime} \mathrm{E}, 2375$ m, 8.IX.2009, leg. M. Schülke (CH09-35), creek valley, devastated secondary deciduous forest, litter \& moss sifted (1 ${ }^{\text {, }}$, coll. Schülke); Yunnan, Lincang Prefecture, Laobie Shan, Wei Bo Shan Pass, $24^{\circ} 08^{\prime} 16^{\prime \prime} \mathrm{N}, 9^{\circ} 42^{\prime} 53^{\prime \prime} \mathrm{E}$, 2375 m, 8.IX.2009, leg. M. Schülke (CH09-35), creek valley, devastated secondary deciduous forest, litter \& moss sifted ( $1+$, coll. Schülke); Yunnan, Baoshan Prefecture, Gaoligong

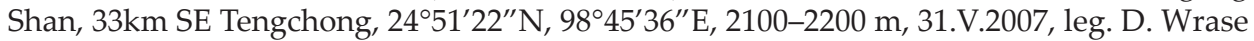
(14b), primary deciduous forest, brook bank, litter, moss sifted (1, coll. Schülke); Yunnan, Baoshan Prefecture, Gaoligong Shan, E pass, 36km SE Tengchong, $24^{\circ} 49^{\prime} 32^{\prime \prime} \mathrm{N}, 98^{\circ} 46^{\prime} 06^{\prime \prime} \mathrm{E}$, 2200 m, 4.VI.2007, leg. M. Schülke (CH07-13), deciduous forest, litter, wood, fungi sifted (1, coll. Schülke); Yunnan, Baoshan Prefecture, Gaoligong Shan, 78 km N Tengchong, $25^{\circ} 44^{\prime} 49^{\prime \prime} \mathrm{N}, 9^{\circ} 33^{\prime} 29^{\prime \prime} \mathrm{E}, 2000 \mathrm{~m}$, 1.IX.2009, leg. M. Schülke (CH09-21), cleft with creek and forest remnant, litter \& dead wood sifted ( 2 + , 1, coll. Schülke, 1 HNHM); Yunnan, Lincang pref., Wuliang Shan, old pass road, $W$ side, small creek valley with primary forest remnant, $24^{\circ} 42^{\prime} 58.6^{\prime \prime} \mathrm{N}, 100^{\circ} 29^{\prime} 52.0^{\prime \prime} \mathrm{E}, 2200$ m, 16.IX.2009, leg. M. Schülke (CH09-47a), litter sifted (2, coll. Schülke); Yunnan prov., Shanzi env., Jizu Shan Mt., along path to summit, $27^{\circ} 57.7-$ $8^{\prime} \mathrm{N}, 100^{\circ} 22.1-23.6^{\prime} \mathrm{E}, 2180-2580$ m, 22-24.VI.2007, leg. J. Hájek \& J. Růžička (CH45-47), sifted detritus and leaves, dense mixed (wet) forest (dominant Pinus, Quercus and Rhododendron) near stream (6, NMPC, 1, coll. Schülke); Yunnan, Baoshan Pref., Gaoligong Shan, nr.

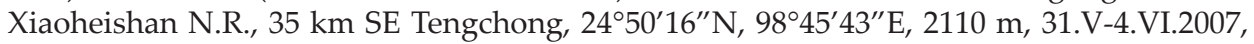
leg. M. Schülke (CH07-11F), deciduous forest, traps (1, coll. Schülke); Sichuan, W-, Daxue

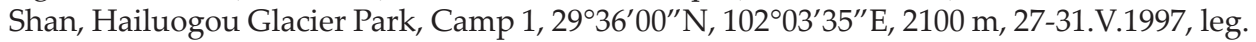
M. Schülke (13), deciduous forest in a river valley, very wet, sifted from litter, moss and mushrooms (1, coll. Schülke, $1 \delta^{`}$, HNHM); Sichuan, SE-, Jinfo Shan, $29^{\circ} 01^{\prime} \mathrm{N}, 107^{\circ} 14^{\prime} \mathrm{E}$, $1750 \mathrm{~m}, 26 . V I .1998$, leg. A. Smetana (C69), sifting of bamboo and broadleaved leaf litter and various floor debris in a secondary broadleaved forest (1, CNCI); Sichuan, SE-, Jinfo Shan, $2^{\circ} 01^{\prime} \mathrm{N}, 107^{\circ} 14^{\prime} \mathrm{E}, 1800 \mathrm{~m}, 27 . \mathrm{VI} .1998$, leg. A. Smetana (C70), sifting moist to wet leaf litter and underlying debris and humus at bases of huge vertical rockblock in original broadleaved forest (1, CNCI, 1, coll. Smetana, NSMT); Sichuan, Emei Shan, Wannian, 1050 m, 19-30.III.1999, leg. W. Schawaller (1, SMNS); Shaanxi, Qinling Shan, 105 km SW Xi'an,

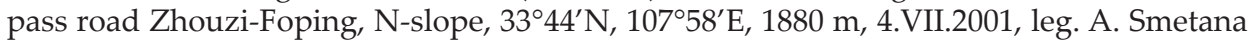
(C92), dripping wet rock wall, sifting of wet debris and vegetation at the base, broadleaved secondary forest (1, CNCI); Shaanxi Prov., Taibai Shan above Houshenzi, 1300-1700 m, 9.VI.-3.VII.1998, leg. P. Jäger \& J. Martens (24, SMNS, 1 ô, MNHP, 1 ô, NMPC, 1, SDEI, 1 , BMNH); Shaanxi, S-, Qinling Shan, pass on Zhouzhi - Foping road, 105 km SW Xi'an, N slope, $33^{\circ} 44^{\prime} \mathrm{N}, 107^{\circ} 58^{\prime} \mathrm{E}, 1880 \mathrm{~m}$, 4.VII.2001, leg. M. Schülke (C01-03), shady rockwall base, moist, sifted (7, coll. Schülke, 1 ${ }^{\lambda}$, HNHM); Hubei, W-, Daba Shan, creek valley 8 km NW Muyuping, $31^{\circ} 29^{\prime} \mathrm{N}, 110^{\circ} 22^{\prime} \mathrm{E}, 1550-1650 \mathrm{~m}, 18$.VII.2001, leg. M. Schülke (C01-16a), deciduous forest, moss, sifted (7, coll. Schülke); Hubei, W-, Daba Shan, creek valley $11 \mathrm{~km} \mathrm{NW}$ Muyuping, 31 $30^{\prime} \mathrm{N}, 110^{\circ} 22^{\prime} \mathrm{E}, 1960 \mathrm{~m}, 18 . \mathrm{VII} .2001$, leg. M. Schülke (C01-17), mixed deciduous forest, sifted (5, coll. Schülke); Hubei, W-, Daba Shan, pass E of Mt. Da Shennongjia, 12

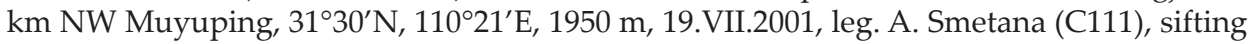
of a pile of grass and various vegetation set up three days prior - broadleaved forest (1, CNCI, 2, coll. Smetana, NSMT); Hubei, W-, Daba Shan, creek valley 8 km NW Muyuping, $31^{\circ} 29^{\prime} \mathrm{N}, 110^{\circ} 22^{\prime} \mathrm{E}, 1550-1650 \mathrm{~m}, 18$.VII.2001, leg. A. Smetana (C115b), sifting of moist to wet leaf litter and various debris accumulated between large rocks on a shady talus slope, mature broadleaved forest (1, CNCI, 5, coll. Smetana, NSMT); Hubei, W-, Daba Shan, pass E of Mt. Da Shennongjia, 12 km NW Muyuping, 31³0’ N, 110²1’E, 1950 m, 18.VII.2001, leg. A. Smetana (C117), sifting of leaf litter and other forest floor debris - broadleaved for- 
est (1, CNCI); Hubei, W-, Daba Shan, pass E of Mt. Da Shennongjia, 12 km NW Muyuping, $31^{\circ} 30^{\prime} \mathrm{N}, 110^{\circ} 21^{\prime} \mathrm{E}$, creek valley $1950-2050 \mathrm{~m}, 19 . \mathrm{VII} .2001$, leg. M. Schülke (C01-13c), mixed deciduous forest, moss, dead wood, mushrooms sifted (1 $\hat{\alpha}$, coll. Schülke); VIETNAM: N-, 7 km NW Sa Pa, 22 $21^{\prime} 49^{\prime \prime} \mathrm{N}, 103^{\circ} 46^{\prime} 49^{\prime \prime} \mathrm{E}, 1850 \mathrm{~m}, 1$.VIII.2015, leg. V. Assing (3a), stream

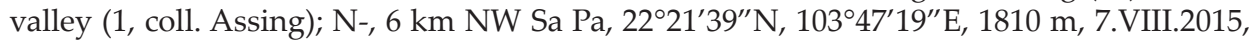
leg. V. Assing (8), pasture margin (1, coll. Assing); N-, $10 \mathrm{~km} \mathrm{NW} \mathrm{Sa} \mathrm{Pa,} 22^{\circ} 22^{\prime} 26^{\prime \prime} \mathrm{N}$, $103^{\circ} 45^{\prime} 27^{\prime \prime} \mathrm{E}, 1850 \mathrm{~m}$, 8.VIII.2015, leg. V. Assing (9), stream valley (2, coll. Assing); N-, pass

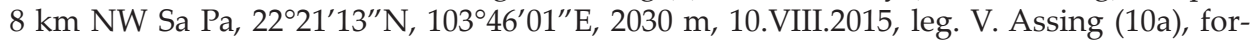
est margin (1, coll. Assing); N-, pass 8 km NW Sa Pa, 22 $21^{\prime} 19^{\prime \prime} \mathrm{N}, 103^{\circ} 46^{\prime} 04^{\prime \prime} \mathrm{E}, 2070 \mathrm{~m}$, 11.VIII.2015, leg. V. Assing (11), degraded primary forest (1, coll. Assing).

Redescription - Measurements (in mm, $\mathrm{n}=10$ ): $\mathrm{HW}=0.65(0.62-0.68) ; \mathrm{TW}=0.63$ (0.58-0.65); $\mathrm{PW}=0.97$ (0.89-1.04); $\mathrm{EW}=1.11$ (1.01-1.22); $\mathrm{AW}=1.11(0.98-1.18) ; \mathrm{HL}=0.51$ (0.47-0.55); $\mathrm{EL}=0.14(0.13-0.15) ; \mathrm{TL}=0.19(0.17-0.20) ; \mathrm{PL}=0.56(0.50-0.61) ; \mathrm{SC}=0.63$ $(0.56-0.72) ; \mathrm{FB}=1.77(1.61-1.88) ; \mathrm{BL}=3.39$ (2.98-3.64). Habitus as in Fig. 95. Forebody moderately shining despite sculpture, abdomen very finely microsculptured with rather large but superficial, indistinct punctures, less lustrous. Forebody reddish dark brown, clypeal area and explanate part of pronotum lighter brown, abdomen medium brown. mouthparts and antennomeres 1-4 reddish medium brown, rest of antennomeres plus legs reddish medium to dark brown. Head with an extremely thin rim on anterior margin, latter imperfectly arched, supraantennal prominences as moderately elevated oblique ridges, disc slightly impressed near eyes. Epistomal suture forming subcircular impression (only very shallowly punctured inside), upper surface without tactile setae, eyes moderately small and very slightly bulging from sideline of head; in dorsal view temples $(1.3 \times$ length of eye) alatiform, laterally rounded and posteriorly constricted to form distinct neck, not separated dorsally by occipital furrow, only by neck being microsculptured rather than punctured. Antennomere 1 flattened club-shaped and lightly microsculptured, segment 2 with broad base and narrowing apically, segment 3 club-shaped, segment 4 small and asymmetrical-incrassate, segments 5-11 with basal dishes, segments 6-10 transverse (more than $1.5 \times$ broader than long). Head and pronotum with mostly alveolate sculpture. Pronotum with lateral portion explanate and slightly up-turned, edge alatiform with sparse short setae; lateral margin unevenly arched with a number of small, irregularly distributed denticles, posterior corners without incision; disc medially with two faint, posteriorly confluent longitudinal ridges, impressed alongside them, lateral parts somewhat impressed. Elytra with membranous posterior margin extending from sutural corner to 5/6 of hind margin. Elytral epipleural ridge present with up-turned lateral alatiform portions, disc longitudinally impressed alongside; shoulders well developed, surface foveolate, in between punctures torulose. Legs short, pro- and mesotibia with several spinulose rows, metatibia with longitudinal ctenidium of spinules in distal half. Abdomen with sides moderately arcuate, second segment with paratergites strongly broadening posteriorly (abdomen appearing constricted at base), those of segments III-VII thin, mesal paratergites broad; posterior edge of tergite VII with thin palisade fringe, median part of sternite VII as in Fig. 181, sternite VIII (Fig. 182), tergites IX (Fig. 183), X (Fig. 184) and aedeagus as in Figs 185-187.

\footnotetext{
Distribution - The known distribution of this species is from the range of the Himalaya to Vietnam.
} 


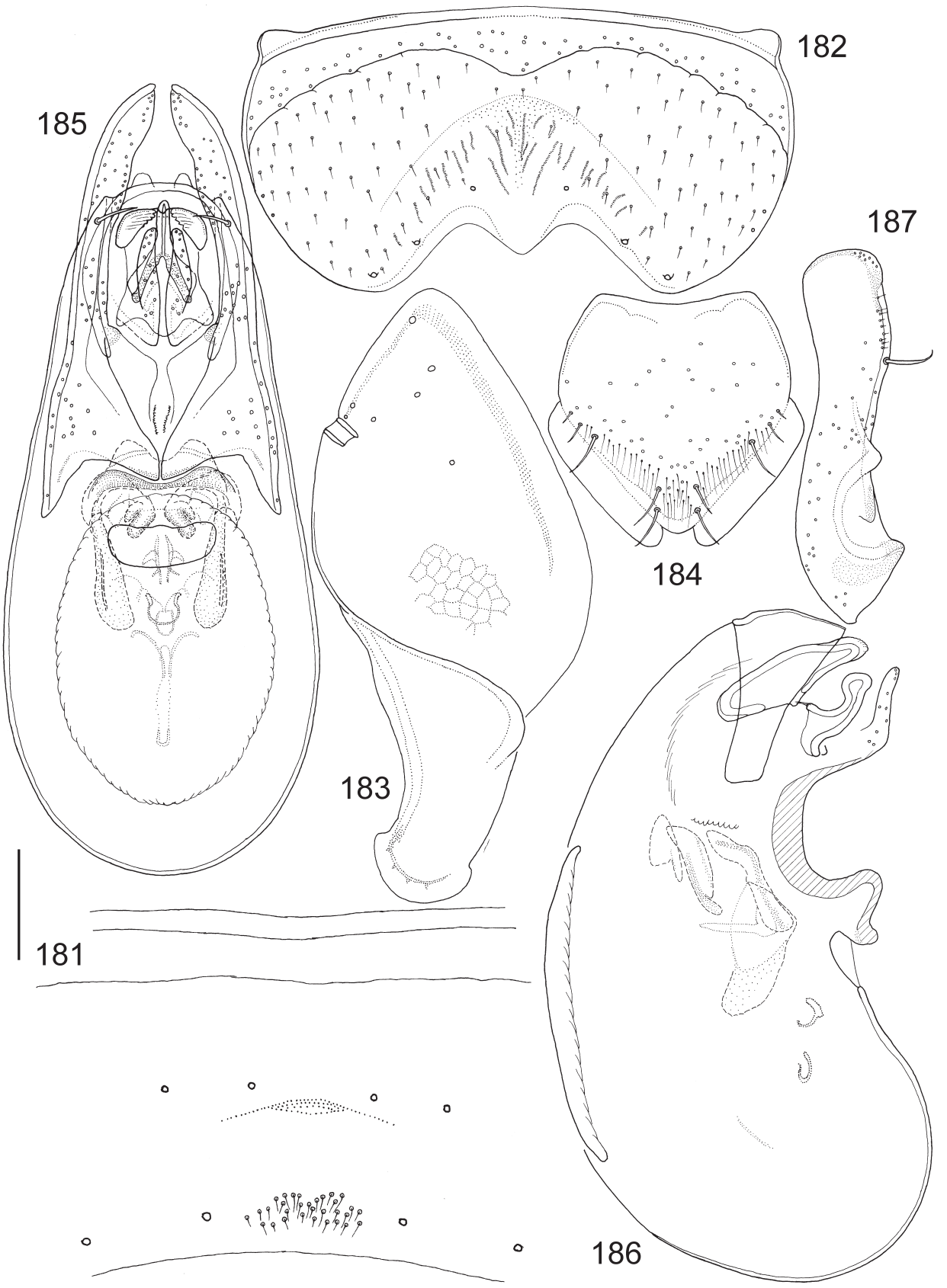

Figs 181-187. Anotylus malaisei (Scheerpeltz, 1965), male. $181=$ median part of sternite VII, 182 = sternite VIII, 183 = tergite IX, 184 = tergite $X, 185$ = aedeagus, frontal view, $186=$ median lobe, lateral view, 187 = paramere, lateral view. Scales: $0.10 \mathrm{~mm}$ for Figs 181, 185-187, $0.11 \mathrm{~mm}$ for Fig. 184, $0.12 \mathrm{~mm}$ for Fig. 183, $0.13 \mathrm{~mm}$ for Fig. 182 
Remarks - The name Oxytelopsis gardneri Paulian, 1940: 58 is preoccupied (not Oxytelus gardneri Cameron 1930: 456, as former became junior secondary homonym in Anotylus (as result of synonymy in MaKRANCzy 2006). The teneral holotype of Oxytelopsis gardneri (label data: Forest Research Institute de Dehra Dun, Nakronda, Dehra Dun, Uttar Pradesh, 22.VI.1936, leg. J. C. M. Gardner) cannot be found, so the present interpretation is based on the original description and a habitus illustration in it. The name was already put into synonymy with O. malaisei in MAKRANCZY (2015), in order to avoid having to be replaced.

\section{Anotylus montanus (Hammond, 1975)}

(Figs 81, 188-194)

Oxytelopsis montana Hammond, 1975: 172; Herman 2001: 1414.

Examined type material - Oxytelopsis montana Hammond - Holotype (ð): “Holo-; type [red margined disc] \Ceylan Central; Horton Plains; 15.II.[19]70 2100 m; Mussard Besuchet Löbl; [on the back, in pencil] $68 \backslash$ Oxytelopsis; montanus sp.n.; P.M. Hammond;

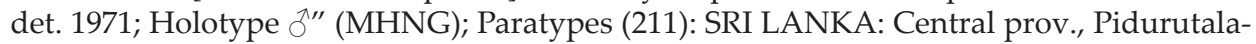
gala, env. 2200 m, 29.I.1970, leg. C. Besuchet, I. Löbl \& R. Mussard (32), tamisages en forêt, versant sud-ouest de la montagne (157, MHNG, 1, NHMW, 1, SMNS, 4, BMNH); Central prov., Nuwara Eliya, à env. 1950 m, 29.I.1970, leg. C. Besuchet, I. Löbl \& R. Mussard (33), tamisages à la limite inférieure de la forêt, au pied du Pidurutalagala (3, MHNG); Central prov., Horton Plains, 2100 m, 15.II.1970, leg. C. Besuchet, I. Löbl \& R. Mussard (68), tamisages en forêt (1, MHNG, 13, BMNH); Central prov., Nuwara Eliya, 1800-1950 m, 15.II.1970, leg. C. Besuchet, I. Löbl \& R. Mussard (69), tamisages à la limite inférieure de la forêt, au pied du Pidurutalagala (20, MHNG, 8, BMNH, 3, FMNH).

Other material - SRI LANKA: Central prov., Horton Plains, 2100 m, 15.II.1970, leg. C. Besuchet, I. Löbl \& R. Mussard (68), tamisages en forêt (1, HNHM, 1 NHMW, 1, NIBR, 1, NMPC, 1, ZMUC).

Redescription - Measurements (in mm, $\mathrm{n}=10)$ : $\mathrm{HW}=0.62(0.59-0.65) ; \mathrm{TW}=0.59(0.55-$ $0.61) ; \mathrm{PW}=0.78$ (0.74-0.81); $\mathrm{EW}=0.79(0.74-0.84) ; \mathrm{AW}=0.91(0.87-0.97) ; \mathrm{HL}=0.53$ (0.50$0.57) ; \mathrm{EL}=0.13(0.12-0.14) ; \mathrm{TL}=0.23(0.22-0.24) ; \mathrm{PL}=0.53$ (0.51-0.56); $\mathrm{SC}=0.40$ (0.36-0.42); $\mathrm{FB}=1.56(1.48-1.66) ; \mathrm{BL}=3.58$ (3.36-3.94). Habitus as in Fig. 81. Forebody somewhat shining despite sculpture, abdomen on segments II-VI without (so shinier than forebody), but segments VII-X with coriaceous microsculpture, former roughly punctured with indistinct borders, latter with less conspicuous punctation, less lustrous. Forebody reddish dark brown, supraantennal area and explanate part of pronotum somewhat lighter, abdomen often more reddish than forebody. Mouthparts and antennomeres 1-4 reddish medium brown, rest of antennomeres plus legs reddish medium to dark brown. Head with anterior margin possessing extremely thin rim, somewhat unevenly arched, anterior clypeal rim very slightly projecting in middle, supraantennal prominences as moderately elevated oblique ridges, disc impressed near eyes. Epistomal suture forming circular impression (inside finer punctured), upper surface without tactile setae, eyes small and slightly bulging; in dorsal view temples $(1.8 \times$ length of eye) moderately alatiform, laterally weakly rounded (very blunt angled) and posteriorly constricted to form distinct neck, however, 


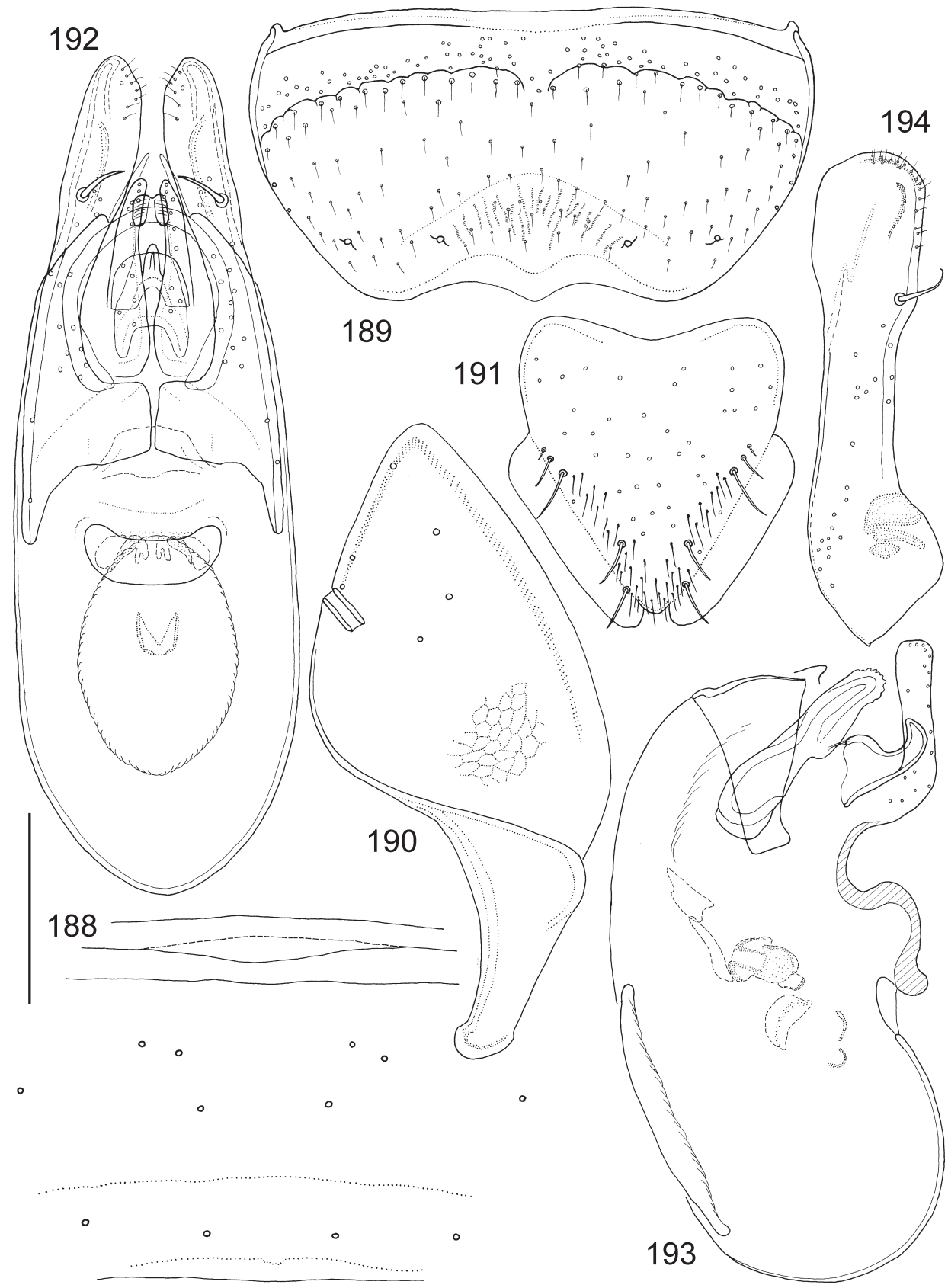

Figs 188-194. Anotylus montanus (Hammond, 1975), male. 188 = median part of sternite VII, 189 = sternite VIII, 190 = tergite IX, 191 = tergite X, 192 = aedeagus, frontal view, $193=$ median lobe, lateral view, 194 = paramere, lateral view. Scales: $0.10 \mathrm{~mm}$ for Figs 192-194, $0.14 \mathrm{~mm}$ for Fig. 191, $0.16 \mathrm{~mm}$ for Fig. 190, $0.19 \mathrm{~mm}$ for Figs 188-189 
dorsal side not separated by occipital furrow, rather by dorsum of neck bearing microsculpture instead of punctation. Antennomere 1 club-shaped and rather smooth, segment 2 ovoid, segment 3 club-shaped, segment 4 small and isodiametricalal, segments 5-11 with basal dishes, articles $6-10$ transverse (about $1.8 \times$ broader than long), segments $9-10$ bulkier than preceding. Head and pronotum with mostly lacunose sculpture. Pronotum with lateral portion explanate and slightly up-turned, edge with sparse short setae, lateral border with several rather prominent angles, gradually transforming into smaller teeth posteriorly, hind margin before corners with shallow incision; disc medially rather convex, parallel longitudinal ridges rather obscured, laterally broadly impressed along sides. Elytra without membranous posterior margin (completely reduced). Elytral epipleural ridge present with up-turned lateral alatiform portions, disc depressed; shoulders moderately developed, surface foveolate, in between punctures indistinctly sculptured. Legs short, pro- and mesotibia with several spinulose rows, metatibia with longitudinal ctenidium of spinules in distal half in distal $2 / 3$. Abdomen with sides very weakly arcuate, second segment with paratergites strongly broadening posteriorly (abdomen appearing constricted at base), those of segments III-VII thin, mesal paratergites broad; posterior edge of tergite VII with thin palisade fringe, median part of sternite VII as in Fig. 188, sternite VIII (Fig. 189), tergites IX (Fig. 190), X (Fig. 191) and aedeagus as in Figs 192-194.

Distribution - The species is only known from the higher altitude areas of Ceylon (Sri Lanka).

Remarks - No more material has become known since the description.

\section{Anotylus nigricans (Cameron, 1933) \\ (Figs 99, 195-201)}

Oxytelopsis nigricans Cameron, 1933: 340; Herman 1970: 413; Hammond 1984: 200; Herman 2001: 1414.

Examined type material - Oxytelopsis nigricans Cameron - Lectotype $(\hat{\sigma}$, here designated): "Lecto-; type [lilac margined disc, curator label] $\backslash$ Type [red margined disc, curator label] \B. N. Borneo; nr. Kinabalu; Tenompok \ pass 4200[‘]; 18-3-1929. \O.; nigricans; Type [underlined] Cam \M. Cameron.; Bequest.; B.M. 1955-147" (BMNH).

Other material - MALAYSIA/BORNEO: Sabah, Mt. Kinabalu National Park Headquarters, 1560-1660 m, river Silau Silau, 24.IV.1987, leg. A. Smetana (B2), sifting of old flood debris (1, FMNH); Sabah, Mt. Kinabalu National Park Headquarters at Liwagu river, $1500 \mathrm{~m}, 25 . \mathrm{IV} .1987$, leg. A. Smetana (B4), sifting old vegetation a debris along a trail in rain forest (1, FMNH); Sabah, Mt. Kinabalu National Park Headquarters, Liwagu river, $1505 \mathrm{~m}, 4$.VIII.1988, leg. A. Smetana (B81) at the edge of a small puddle on the trail in rainforest (1, FMNH); Sabah, Mt. Kinabalu National Park Headquarters, Liwagu river, 1490 m, 5.VIII.1988, leg. A. Smetana (B84) sifting of fermenting fruits and fallen flowers of a "kerosene tree" in rain forest (81, FMNH, 1, NIBR); Sabah, Mt. Kinabalu National Park Headquarters, Liwagu river, base of large vertical rock block, 1490 m, 10.VIII.1988, leg. A. Smetana (B97), sifting of leaf litter, various debris and humus accumulated (11, FMNH), same but 3.IX.1988 (B174) rain forest, fermenting fruits and fallen flowers of a "kerosene tree", sifted repeatedly after a month, but some fresh fruits present there also (31, FMNH, 1, MNHP); Sabah, Mt. Kinabalu National Park Headquarters, Liwagu river Trail, 1520 m, 11.VIII.1988, leg. A. Smetana (B100), sifting of layers of small fallen fleshy flowers (from a 
tree) on forest floor and debris under (22, FMNH, 1, ISNB, 1, AMNH, 1, SMNS, 1, SDEI), same, but 12.VIII.1988 (B106) sifting of fermenting fruits and fallen flowers of a "kerosene tree" in rain forest (4, FMNH); Sabah, Mt. Kinabalu, 1850 m, Liwagu Trail, 27.IV.1987, leg. D. Burckhardt \& I. Löbl (6), tamisage de feuilles mortes et mousses près de la rivière, (1, MHNG); Sabah, Mt. Kinabalu, Silau-Silau Trail, 1560 m, 28.IV.1987, leg. D. Burckhardt \& I. Löbl (7b), tamisage de feuilles mortes et mousses (1, MHNG); Sabah, Mt. Kinabalu, 1500 m, Liwagu Trail, section 2, forêt de Lithocarpus-Podocarpus, 21.V.1987, leg. D. Burckhardt \& I. Löbl (34a), tamisage de débris végétaux sur une pente partiellement débroussaillée, (18, MHNG, 2, HNHM); Sabah, E of Mt. Kinabalu, 1150 m, route Ranau-Kota Kinabalu, 24.V.1987, leg. D. Burckhardt \& I. Löbl (40), tamisage de bois pourri avec champignons et débris végétaux, à proximité d'une plantation et dans un ravin boisé, (2, MHNG); INDONESIA/SULAWESI: SE-, ins. Buton, Wakarumba [454’S, $122^{\circ} 50^{\prime}$ E], 3-7.II.1994, leg. M. Štrba \& I. Jeniš (1 ㅇ, NHMW).

Redescription - Measurements (in $\mathrm{mm}, \mathrm{n}=10)$ : $\mathrm{HW}=0.79(0.76-0.83) ; \mathrm{TW}=0.84$ (0.80-0.90); $\mathrm{PW}=1.16$ (1.10-1.19); $\mathrm{EW}=1.29$ (1.23-1.35); $\mathrm{AW}=1.26(1.20-1.32) ; \mathrm{HL}=0.58$ $(0.56-0.61) ; \mathrm{EL}=0.17(0.16-0.18) ; \mathrm{TL}=0.22(0.20-0.23) ; \mathrm{PL}=0.66(0.63-0.68)$; $\mathrm{SC}=0.78(0.75-$ $0.84) ; \mathrm{FB}=2.14(2.07-2.22) ; \mathrm{BL}=3.96$ (3.85-4.04). Habitus as in Fig. 99. Forebody moderately shining despite sculpture, abdomen very finely and densely microsculptured and extremely shallowly punctured, without lustre. Body blackish dark brown, usually with strong reddish tint but clypeal area lighter, reddish (alatiform portion of pronotum also lighter for transparency); mouthparts and antennae medium to dark brown. Head rather transverse with anterior margin forming an arch more or less truncate in middle, an extremely thin rim, supraantennal prominences as moderately elevated oblique ridges, disc slightly impressed near eyes, middle of vertex slightly elevated. Epistomal suture forming subcircular impression (less sculptured inside), upper surface without tactile setae, eyes medium large, moderately convex; in dorsal view temples $(1.3 \times$ length of eye) alatiform, laterally angled and posteriorly constricted to form distinct neck, however, dorsal side not separated by occipital furrow, rather by dorsum of neck bearing microsculpture instead of punctation. Antennomere 1 flattened club-shaped and finely microsculptured, segment 2 oval, segment 3 club-shaped, segment 4 almost round, segments 5-11 with basal dishes, segments 6-10 transverse (about $2 \times$ broader than long). Head and pronotum with mostly alveolate sculpture (transitional to lacunose). Pronotum with lateral portion explanate and slightly up-turned, edge alatiform with sparse short setae; lateral border uneven and with a number of small, irregularly distributed denticles, posterior margin strongly incised before corners; disc medially with two parallel and posteriorly almost confluent longitudinal ridges, posteriorly impressed alongside them, laterally impressed around middle of sides. Elytra with membranous posterior margin extending from sutural corner to $5 / 6$ of hind margin. Elytral epipleural ridge present with up-turned lateral alatiform portions, disc longitudinally impressed alongside; shoulders well developed, surface foveolate, in between punctures torulose. Legs short, pro- and mesotibia with several spinulose rows, metatibia with longitudinal ctenidium of spinules in distal half. Abdomen with sides gently arcuate, second segment with paratergites strongly broadening posteriorly (abdomen appearing constricted at base), those of segments III-VII thin, mesal paratergites broad; posterior edge of tergite VII with thin palisade fringe, median part of sternite VII as in Fig. 195, sternite VIII (Fig. 196), tergites IX (Fig. 197), X (Fig. 198) and aedeagus as in Figs 199-201. 


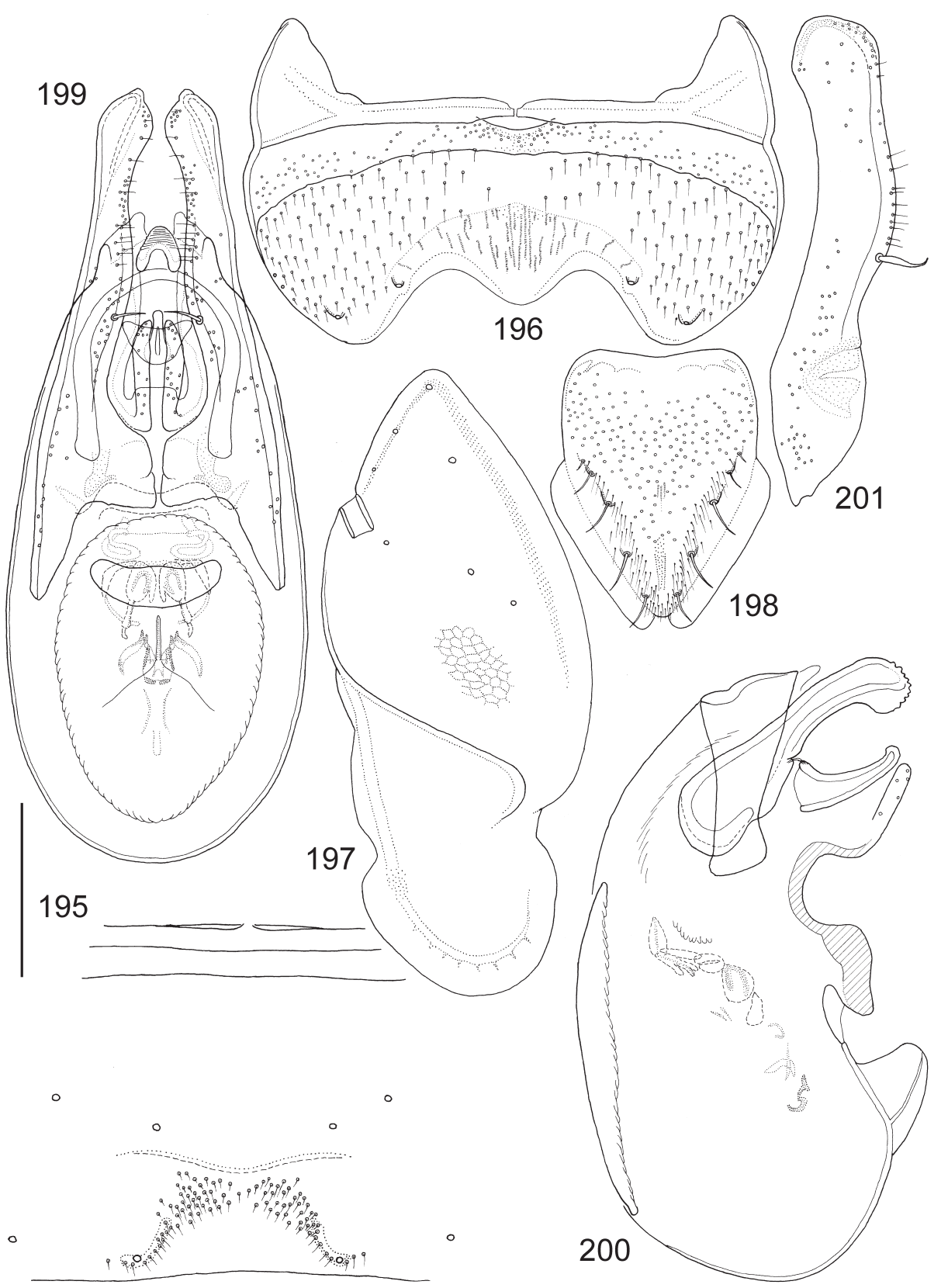

Figs 195-201. Anotylus nigricans (Cameron, 1933), male. 195 = median part of sternite VII, 196 = sternite VIII, 197 = tergite IX, 198 = tergite X, 199 = aedeagus, frontal view, $200=$ median lobe, lateral view, 201 = paramere, lateral view. Scales: $0.20 \mathrm{~mm}$ for Figs 195, 199-201, $0.24 \mathrm{~mm}$ for Fig. 198, $0.26 \mathrm{~mm}$ for Figs 196-197 
Distribution - The species is known from northern Borneo, but a single female specimen was found from southeast Sulawesi as well. This latter record requires verification by more material.

Remarks - It is unknown why P. M. Hammond left the type of this species unlabelled - the only primary type specimen of this group in BMNH without his label. The original description refers to two specimens, the whereabouts of the second specimen remain unknown. The species was found in flood debris and leaf litter plus other decaying vegetable debris (e.g. fallen fleshy flowers).

\section{Anotylus pseudopsinus (Fauvel, 1895)}

(Figs 94, 202-208)

Oxytelopsis pseudopsina Fauvel, 1895: 201; Cameron 1930: 206; Herman 1970: 413; Hammond 1975: 169, 170; Hammond 1984: 200; Coiffait 1984: 151; Herman 2001: 1414.

Oxytelopsis rufotestacea Cameron, 1925: 176, syn. nov.; Herman 1970: 413; Herman 2001: 1415.

Oxytelopsis brevipennis Bernhauer, 1926: 313, syn. nov. (preoccupied); Herman 1970: 413; Herman 2001: 1413. (see remark)

Oxytelopsis chapmani Cameron, 1934: 32, syn. nov.; Herman 1970: 413; Herman 2001: 1413. Oxytelopsis nigripennis Cameron, 1934: 32, syn. nov.; Herman 1970: 413; Herman 2001: 1414. Oxytelopsis cassagnaui Coiffait, 1982: 163, syn. nov.; Coiffait 1984: 50; Herman 2001: 1413.

Examined type material - Oxytelopsis pseudopsina Fauvel - Lectotype ( $\sigma^{1}$, here designated): “Lecto-; type [lilac margined disc, curator label] \[Mt.] Carin; Asciuii Ghecù [19 $19^{\circ} \mathrm{N}$, 96²4.5’E]; 1400-1500 m.; L. Fea. III-IV.[18]88. \ pseudopsina; Fvl. \ R.I.Sc.N.B. 17.479; Oxytelopsis; Coll. et det. A. Fauvel \Syntype \Oxytelopsis; pseudopsina Fvl.; P.M. Hammond; det. 1972; Lectotype \ Anotylus; pseudopsinus (Fauvel); det. Makranczy, 2012" (ISNB); Paralectotypes (2): "Para-; lecto-; type [light blue margined disc] \Carin; Asciuii Ghecù; 1400-1500 m.; L. Fea. III-IV.[18]88. \ Coll. et det. A. Fauvel; Oxytelopsis; pseudopsina; Fauv.; R.I.Sc.N.B. $17.479 \backslash$ Oxytelopsis; pseudopsina Fvl.; P.M. Hammond; det. 1972; Paralectotype \Anotylus; pseudopsinus (Fauvel); det. Makranczy, 2015" (2 , ISNB); Oxytelopsis rufotestacea Cameron - Syntype (+): "Para-; lecto-; type [light blue margined disc] \ Para-; type [yellow margined disc] \Prinsen Eil.[and = Pulau Panaitan]; 21.I.1922; Damm. [erman] \Oxytelopsis; rufotestacea; Cam. \M. Cameron.; Bequest.; B.M. 1955-147. \Oxytelopsis; rufotestacea Cam.; P.M. Hammond; det. 1970; Paralectotype" (BMNH); Syntype (ㅇ, teneral): "Prinsen Eil.[and]; 21.I.1922; Damm.[erman] \Oxytelopsis; rufotestacea; Cam; Type. \Type [red card]" (RMNH); Oxytelopsis chapmani Cameron - Syntype ( + ): "Lecto-; type [lilac margined disc, curator label] \Type [red margined disc] \Hoa-Binh.; Tonkin. $193 \backslash$ O.; chapmani; Type [underlined] Cam. \ M. Cameron.; Bequest.; B.M. 1955-147. \ Oxytelopsis; chapmani Cam.; P.M. Hammond; det. 1970; Lectotype" (BMNH); Oxytelopsis nigripennis Cameron - Syntype (+): "Lecto-; type [lilac margined disc, curator label] \Type [red margined disc] \Hoa-Binh.; Tonkin. $193 \backslash$ O.; nigripennis Type [underlined] Cam. \ M. Cameron.; Bequest.; B.M. 1955-147. \Oxytelopsis; nigripennis Cam.; P.M. Hammond; det. 1970; Lectotype" (BMNH), Syntype (o): "Hoa-Binh; Tonkin \Cotype \Oxytelopsis; nigripennis; Cam. \Oxytelopsis; nigripennis; Cameron \Coll. W. Chapman; in Coll. P. Griveau; MHNG-2007 \nigripennis; Cam." (MHNG); Oxytelopsis brevipennis Bernhauer 
- Holotype (ठ̋): “[leg.] L.J. Toxopeus; Buru, Station 9; 12. VI.[19]’21 \Virgin jungle;; decaying leaves \Oxytelopsis; brevipennis; Brnh. Typus unic[us] \brevipennis; Bernh. Treubia; 1926, p. $313 \backslash$ Chicago NHMus; M. Bernhauer; Collection \Holo-; type [red margined disc, curator label] \Oxytelopsis; brevipennis Bnh; P.M. Hammond; det. 1974; Holotype $\widehat{\sigma} \backslash$ FMNH-INS; 0000063472 \Photographed; D. Le 2011; EMu catalog" (FMNH).

Other material - VIETNAM: Tuyen Quang prov., NaHang Reserve, 300 m, 1620.V.1997, leg. S. Peck (97-8), rainforest, FIT (1, MHNG); Bien-ho, Плантация Кинкинь, 17.X.1979, probe 0-10cm (1 §̃, 2, coll. Ryvkin); N-, Ninh Binh Pr., 90 km SW Hanoi, Cuc Phuong NP, primates resc. centr., $190 \mathrm{~m}, 2^{\circ} 14^{\prime} 24^{\prime \prime} \mathrm{N}, 105^{\circ} 42^{\prime} 53^{\prime \prime} \mathrm{E}, 25 . \mathrm{IV} .2012$, leg. A. Weigel \& Li-Fa (2, NKME); Ninh Binh Prov., Res. Cuc-Phüöng, 200 m, 14.X.1963, leg. T. Pócs, dans forêt tropic. pluv., à la lumière (1, HNHM); Hanoi, 20-30.IV.1991, leg. E. Jendek, at light (3, NHMW); Hanoi (1, ISNB); S-, 40 km NW An Khe, Buon Luoi, 14¹0’ N, 108 $30^{\prime}$ E, 620-750 m, 28.III-12.IV.1995, leg. P. Pacholátko \& L. Dembický (4, NHMW); S-, Nam Cat Tien Nat. Park, 1-15.V.1994, leg. P. Pacholátko \& L. Dembický (2, NHMW); Muong son, 8. XII.1971, leg. Gy. Topál (268), sifted litter (1, HNHM); Ha Son Binh, Hoa Binh, 30.I.1986, leg. S. Mahunka \& J. Oláh (Berlesate No. 23) (1, HNHM), Lam Dong prov., Duc Me (Maria stream), 15 km S Bao Loc, 700 m, 23.X.1988, leg. S. Mahunka \& T. Vásárhelyi (No. 372), at

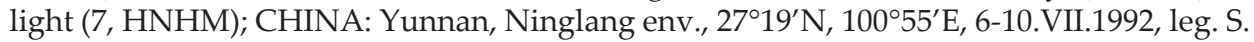
Bečvář (1, MHNG); INDIA: Kerala, Cardamom Hills, Mundakayam, 100 m, 9.XI.1972, leg. C. Besuchet, I. Löbl \& R. Mussard (16), tamisages dans plantation d'hévéas (2, MHNG); Kerala, Cardamom Hills, Valara Fall, à 46 km SW Munnar, 450-500 m, 25.XI.1972, leg. C. Besuchet, I. Löbl \& R. Mussard (49), tamisages dans la forêt près de la rivière (3, MHNG); Kerala, Nelliampathi Hills (NW Anaimalai Hills), Kaikatty, 900 m, 30.XI.1972, leg. C. Besuchet, I. Löbl \& R. Mussard (58), tamisages den forêt près d'un ruisseau (33, MHNG); Madhya Pradesh, Mahadeo Hills, 4.5 km SW Pachmarhi, Vanshree Vihar $22^{\circ} 26^{\prime} 34^{\prime \prime} \mathrm{N}, 78^{\circ} 23^{\prime} 25^{\prime \prime} \mathrm{E}$, 950 m, 16.X.2000, leg. G. Cuccodoro (1a), sifting flood debris at stream (32, MHNG); Madhya Pradesh, Mahadeo Hills, 4.5 km SW Pachmarhi, Vanshree Vihar $22^{\circ} 26^{\prime} 34^{\prime \prime} \mathrm{N}, 78^{\circ} 23^{\prime} 25^{\prime \prime} \mathrm{E}$, 950 m, 16.X.2000, leg. G. Cuccodoro (1b), sifting leaf litter near stream (15, MHNG); Madhya Pradesh, Mahadeo Hills, 5.5 km SW Pachmarhi, Tridhara $22^{\circ} 26^{\prime} 09^{\prime \prime} \mathrm{N}, 78^{\circ} 23^{\prime} 01^{\prime \prime} \mathrm{E}, 900$ $\mathrm{m}, 18 . X .2000$, leg. G. Cuccodoro (3b), sifting moist leaf litter on rocks in gallery forest (10, MHNG); Goa, Western Ghats, 1.5 km E Tambdi Suria Temple, $15^{\circ} 26^{\prime} 27^{\prime \prime} \mathrm{N}, 74^{\circ} 15^{\prime} 49^{\prime \prime} \mathrm{E}, 350$ $\mathrm{m}$, 31.X.2000, leg. G. Cuccodoro (3b), sifting vegetable debris in clearing (1, MHNG); Orissa [Odisha], Daitari, 24.XII.1966, leg. Gy. Topál (54), extracted from mosses (1, HNHM), same but (105), 2.I.1967 (1, HNHM); Orissa, Jajpur, Jajpur-Kendujhar Distr., Daitari, 23.XI.1967, leg. Gy. Topál (925), sifted litter (2, HNHM); Orissa, Jajpur, Jajpur-Kendujhar Distr., Daitari, 25.XI.-4.XII.1967, leg. Gy. Topál (941), sifted bark of tree (2, HNHM); Orissa, Jajpur, Jajpur-Kendujhar Distr., Daitari, 4.XII.1967, leg. Gy. Topál (1012), from under bark (1, HNHM); Uttar Pradesh, Rishikesh, Laxman-Bridge, 3-4.VII.1989, leg. A. Riedel (1, SMNS); N-, Uttar Pradesh, 15-22 km N Rishikesh, 8.VIII.1989, leg. Hiermeier (47) (1, NHMW); Kumaon (Uttar Pradesh), Bhim Tal, ca. 1500 m, 4.X.1979, leg. I. Löbl (1), lisière d'une forêt secondaire, versant est, assez sec, tamisage (3, MHNG); Kumaon (Uttar Pradesh), Bhim Tal, 1550 m, 5.X.1979, leg. I. Löbl (2b) sous des pierres au bord d’un ruisseau (1, MHNG); Kumaon (Uttar Pradesh), Kathgodam près Haldwani, 600 m, 6.X.1979, leg. I. Löbl (3), ravin boisé, tamisage de feuilles mortes près d'un ruisseau (97, MHNG); Kumaon (Uttar Pradesh), entre Bhim Tal et Sat Tal, ca. 1500 m, 7.X.1979, leg. I. Löbl (4), forêt secondaire, versant nord, tamisage de feuilles mortes (1, MHNG); Kumaon (Uttar Pradesh), Garija à 10 km de Ramnagar (Corbett National Park), 450 m, 15.X.1979, leg. I. Löbl (12b) tamisage de feuilles mortes dans un ravin profond (1, MHNG); Garhwal (Uttar Pradesh), route pour 
Mussoorie, 1300 m, 18.X.1979, leg. I. Löbl (14), versant sud, ravin boisé, tamisage de feuilles et branches mortes, humide (3, MHNG); Garhwal (Uttar Pradesh), 20 km au sud de Chamba, 1150 m, 20.X.1979, leg. I. Löbl (16), ravin avec une source, gravier avec les racines de l'herbe et accumulation du bois pourri (9, MHNG); Garhwal (Uttar Pradesh), $4 \mathrm{~km}$ au sud de Bhatwari, 1400 m, 23.X.1979, leg. I. Löbl (22), lisière d'une foret très dégradée, tamisage de mousses et fougères (2, MHNG); Garhwal (Uttar Pradesh), entre Tehri et Srinagar, à 20 $\mathrm{km}$ de Srinagar, ravin, 900 m, 25.X.1979, leg. I. Löbl (24 and 24c), tamisage, terreau audessus de buissons (14, MHNG); Garhwal (Uttar Pradesh), entre Srinagar et Devaprayag, à 16 km de Srinagar, 550 m, 29.X.1979, leg. I. Löbl (29), au bord d'une rivière, tamisage de terreau très humide (2, MHNG); Garhwal (Uttar Pradesh), 22 km N de Rishikesh, $450 \mathrm{~m}$, 30.X.1979, leg. I. Löbl (31), versant sud, ravin boisé, tamisage de feuilles et branches mortes, humide (5, MHNG); Himachal Pradesh, Shivalik Hills, 6km SE Nahan, 800-900 m, 6.X.1996, leg. Schulz \& Vock (1, coll. Assing); [Uttarakhand] Siwaliks, Nakraunda [30 $14^{\prime} \mathrm{N}, 78^{\circ} 08^{\prime} \mathrm{E}$ ], 8.X.1922, leg. M. Cameron (1, NMPC); [Uttarakhand] Kolhu Khet Gad. Mussoorie district, 1.XI.1921, leg. M. Cameron (1, MHNG); Uttarakhand, left side of Kosi River, 5 km N Ramnagar, $29.432^{\circ} \mathrm{N}, 79.140^{\circ} \mathrm{E}, 19-23 . V I .2011$, leg. A. Shavrin (1, coll. Shavrin); NE-, Assam/ Arunachal Pr. border, Bhalukpong, $27^{\circ} 00^{\prime} 48^{\prime \prime} \mathrm{N}, 9^{\circ} 39^{\prime} 08^{\prime \prime} \mathrm{E}, 150 \mathrm{~m}, 1-8 . \mathrm{V} .2012$, leg. L. Dembický, Flight Interception Trap (1, ZFMK), same but sifting + Winkler app. extraction (1, ZFMK); Assam, Umrongso env. $700 \mathrm{~m}, 2^{\circ} 27^{`} \mathrm{~N}, 9^{\circ} 43^{`} \mathrm{E}, 3-8 . \mathrm{VI} .2002$, leg. M. Trýzna \& P. Benda (9, coll. Schülke); Assam, Gauhati, 200 m, 20.X.1978, leg. C. Besuchet, I. Löbl (22), tamisage au pied d'une paroi de terre, dans une colline plus ou moins boisée (3, MHNG); Assam, Manas (Manas wildlife sanctuary), 200 m, 21.X.1978, leg. C. Besuchet, I. Löbl (23), tamisage dans la forêt près des bungalows, sol sablonneux (13, MHNG); Assam, Manas, 200 m, 22.X.1978, leg. C. Besuchet, I. Löbl (24a), sous des écorces (1, MHNG), same but (24c), tamisage en forêt (4, MHNG); Meghalaya, Garo Hills, Songsak, 400 m, 2.XI.1978, leg. C. Besuchet, I. Löbl (38c), tamisage en forêt, principalement sous de bambous (2, MHNG); Meghalaya, Garo Hills, Rongrengiri, 400 m, 3.XI.1978, leg. C. Besuchet, I. Löbl (39b), tamisage en forêt, au pied de grands arbres (3, MHNG); Meghalaya, Khasi Hills, Nongpoh, forêt au-dessus du village, 700 m, 5.XI.1978, leg. C. Besuchet, I. Löbl (42b), tamisage dans la forêt, versant nord, au pied de rochers et de grands arbres (7, MHNG); Meghalaya, $1 \mathrm{~km}$ E of

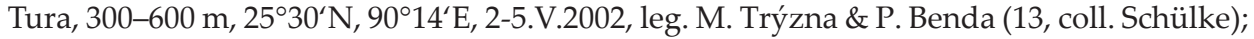
West Bengal, Darjeeling distr., Teesta, $3 \mathrm{~km}$ en amont du village, $250 \mathrm{~m}, 10 . X .1978$, leg. C. Besuchet, I. Löbl (7 and $7 \mathrm{~b}$ ), tamisage en lisière de forêt, au pied de grands arbres avec lianes (13, MHNG); West Bengal, Darjeeling distr., Sukna, 200 m, 7.X.1978, leg. C. Besuchet, I. Löbl (2b), tamisage en forêt (8, MHNG); West Bengal, Darjeeling distr., entre Teesta et Rangpo, à $11 \mathrm{~km}$ de Teesta, $350 \mathrm{~m}, 12 . X .1978$ leg. C. Besuchet \& I. Löbl (11b), sous des écorces (3, MHNG); West Bengal, Darjeeling distr., Tonglu, versant nord, 2700 m, 16.X.1978 leg. C. Besuchet \& I. Löbl (17), tamisages den forêt (2, MHNG); West Bengal, Darjeeling distr., Singla, 300 m, 18.X.1978, leg. C. Besuchet, I. Löbl (18b), tamisage dans une forêt secondaire de tecks (1, MHNG); SRI LANKA: Lindula, $10 \mathrm{~km}$ WSW Nuwara Eliya, leg. G. Benick, 29.III.1973, Quelle (1, coll. Schülke); Dickoya, 20 km WSW Nuwara Eliya, leg. G. Benick, 28.III.1973, Quelle im Fels, gesiebt (1, coll. Schülke); Central prov., Kandy, env. 600 m, forêt près du "Chalet Guesthouse", 15.I.1970, leg. C. Besuchet, I. Löbl \& R. Mussard (3c), tamisages (1, MHNG); Central prov., Kandy, env. 600 m, Udawattekele Sanctuary, 19.I.1970, leg. C. Besuchet, I. Löbl \& R. Mussard (11), tamisages en forêt vierge (3, MHNG); Central prov., au-dessus de Talatuoya, entre 850 et 1000 m, 27.I.1970, leg. C. Besuchet, I. Löbl \& R. Mussard (27a), tamisages dans des restes de forêt (4, MHNG); Central prov., Hanguranketa, 750 m, 27.I.1970, leg. C. Besuchet, I. Löbl \& R. Mussard (28), écorces et Poly- 
pores d'une vieille souche (3, MHNG); Central prov., Mahaweli Ganga, à 7 mi en aval de Kandy, env. 450 m, 30.I.1970, leg. C. Besuchet, I. Löbl \& R. Mussard (34), au bord du fleuve, sur le sable humide au pied des graminées (1, MHNG); Central prov., Ginigathena, env. 650 m, au bord du Mahaweli Ganga, 9.II.1970, leg. C. Besuchet, I. Löbl \& R. Mussard (56), sur le sable humide (1, MHNG); Central prov., Kandy, env. 700 m, collines boisées au sud du lac, 14.II.1970, leg. C. Besuchet, I. Löbl \& R. Mussard (67b), tamisage de feuilles mortes accumulées dans un grand trou (24, MHNG); THAILAND: S-, Yala prov., Betong distr.,

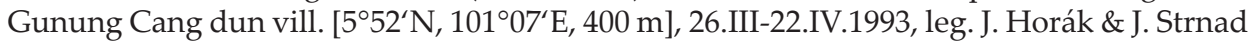
(91, NHMW); Phang-nga prov., Takuapa distr., Khao Lak, 08³7.623`N, 98 ${ }^{\circ} 15.091^{`} \mathrm{E}, 50 \mathrm{~m}$, 23.VIII.-2.IX.2010, leg. A. Skale (NHMW); Prov. Phitsanulok, Thung Sa-Laeng Luang N.P., 31.V.2001, leg. E. Horváth \& Gy. Sziráki (21), at light (1, HNHM); Khao Yai NP [several small streams, very shaded, with sandy bottom, approx. $\left.14^{\circ} 22^{\prime} 26^{\prime \prime} \mathrm{N}, 101^{\circ} 21^{\prime} 42^{\prime \prime} \mathrm{E}\right], 14$. XI.1988, leg. M. Jäch (3) (1, NHMW); Mae Ping, 5-10.IX.1991, leg. H. Malicky, licht (2, NHMW); W-, Sai Yok Yai NP, NW Kanchanaburi, 3.XII.1999, leg. M. Jäch (8) (1, NHMW); Soppong, $19^{\circ} 29^{\prime} \mathrm{N}, 9^{\circ} 18^{\prime} \mathrm{E}, 750 \mathrm{~m}, 13 . \mathrm{V} .1993$, leg. L. Bocák (3, SMNS); Soppong, $19^{\circ} 29^{\prime} \mathrm{N}$, 98¹8`E, 750 m, 13.V.1993, leg. V. Kubáň (4, SMNS); Chiang Mai, Doi Suthep, versant nord, 1050 m, 5.XI.1985, leg. D. Burckhardt \& I. Löbl (10), ravin très humide, tamisage de bois pourri, des écorces et champignons (1, MHNG); Chiang Mai, Doi Inthanon, près des maisons du „Forestry Department“, 1250 m, 6.XI.1985, leg. D. Burckhardt \& I. Löbl (13), tamisage de débris végétaux au bord d'un ruisseau (1, MHNG); Phetchaburi, Kaeng Krachan National Park, 200 m, aux environs de „Headquarters“, 16.XI.1985, leg. D. Burckhardt \& I. Löbl (23), tamisage de débris végétaux à la lisière de la forêt (57, MHNG); Phetchaburi, Kaeng Krachan National Park, 300-400 m, 25 à 30 km de „Headquarters“, 17.XI.1985, leg. D. Burckhardt \& I. Löbl (24), tamisage au pied de gros arbres (24, MHNG); Phetchaburi prov., Kaeng Krachan National Park, 450 m, à 30 km de „Headquarters“, 18.XI.1985, leg. D. Burckhardt \& I. Löbl (25), tamisage au pied de gros arbres (1, MHNG); Phetchaburi, Kaeng Krachan National Park, 450 m, à 35 km de „Headquarters“, 19.XI.1985, leg. D. Burckhardt \& I. Löbl (26), tamisage de débris végétaux dans la forêt (36, MHNG); Chanthaburi, Khao Sabap National Park, environs de Phliu Waterfalls, 150-300 m, 23-24.XI.1985, leg. D. Burckhardt \& I. Löbl (27a), tamisage de débris végétaux et de mousses à proximité de cours d'eau (3, MHNG); NE Bangkok, Khao Yai National Park, aux environs de "Headquarters", 750850 m, 26.XI.-3.XII.1985, leg. D. Burckhardt \& I. Löbl (28), sous les écorces (3, MHNG); NE Bangkok, Khao Yai National Park, aux environs de "Headquarters", 750-850 m, 26.XI.-3. XII.1985, leg. D. Burckhardt \& I. Löbl (28b), tamisage de débris végétaux (26, MHNG); NE Bangkok, Khao Yai National Park, Collines E Heo Suwat Waterfalls, 800 m, 1.XII.1985, leg. D. Burckhardt \& I. Löbl (30a), tamisage de débris végétaux à la lisière de la forêt, près d'un merécage et près d'un ruisselet (3, MHNG); Chiang Mai prov., Chiang Dao distr., Doi Chiang Dao Wildlife Sanctuary, 510 m, 22.IX.-25.X.1990, leg. P. Schwendinger, pitfall traps (2, MHNG); Chiang Mai prov., Doi Angkhang, 10 km W Fang, 1500 m, 30.X.1987, leg. P. Schwendinger, (2, MHNG); Chiang Mai prov., Doi Suthep, 1180 m, I-II.1986, leg. P. Schwendinger, pitfall trap (1, MHNG); Chiang Mai prov., Doi Inthanon, 1020 m, 17.II.1987, leg. P. Schwendinger (1, MHNG); Chiang Rai prov., 10 km W Wiang Pa Pao, Ban Huay Ya Sai, 780 m, 28.I.1988, leg. P. Schwendinger (6, MHNG); Chiang Rai prov., Tham Pla, 500 m, 31.X.1991, leg. P. Schwendinger (3, MHNG); Mae Hong Son prov., Pong Düat, 750 m, 10. IV.1987, leg. P. Schwendinger (1, MHNG); Chanthaburi prov., Nam tok Phliu, 50-100 m, 20.XI.1991, leg. P. Schwendinger (1, MHNG); Trang prov., Khao Chong Botanical Garden, 26.IX.2003, leg. A. Orosz \& Gy. Sziráki (no. 30), at light at staff center (1, HNHM); Trang prov., Khao Chong Botanical Garden at Khao Chong river, 29.IX.2003, leg. A. Orosz \& Gy. 
Sziráki (no. 39), at black light (1, HNHM); LAOS: Khammouan prov., Ban Khoun Ngeun, 300 m, 16.V.-6.VI.2007, leg. M. Štrba (5, SMNS); S-, Prov. Champasak, ca. 50 km S Pakse, Umg. Ban Phatoumphone, burnt clearings, 50-100 m, 23-24.V.1996, leg. H. Schillhammer (1a), at light (2, NHMW); ; CAMBODIA: Silhanoukville, 5 m, 1.-13.I.2009, leg. S. \& M. Murzin (1 ô, coll. Schülke); Paifin, 200 m, 11-16.V.2009, leg. S. Murzin (1 ô, coll. Schülke); MYANMAR: Sagaing Division, Alaungdaw Katthapa NP, ca. 1.5 km SW Log Cabin Camp, along Pagoda Road, $22^{\circ} 18.902^{\prime} \mathrm{N} 94^{\circ} 28.060^{\prime} \mathrm{E}$, ca. 400 m, 6.V.2003, leg. H. Schillhammer et al. (108), semi-evergreen forest close to Pagoda Stream; sifting of moist leaf litter in small ravines (10, NHMW); Sagaing Division, Alaungdaw Katthapa NP, around Ku Mara monastery, $22^{\circ} 18.560^{\prime} \mathrm{N}, 9^{\circ} 27.679^{\prime} \mathrm{E}$, ca. $400 \mathrm{~m}, 8$.V.2003, leg. H. Schillhammer et al. (117), sifting of moist leaf litter among stones in dried-up stream-bed, shaded by large bushes (11, NHMW); Sagaing Division, Alaungdaw Katthapa NP, Khaung Din Stream, between $22^{\circ} 18.360^{\prime} \mathrm{N} 94^{\circ} 25.937^{\prime} \mathrm{E}$ and $22^{\circ} 19.650^{\prime} \mathrm{N} 94^{\circ} 25.768^{\prime} \mathrm{E}$, ca. $450 \mathrm{~m}, 11.5 .2003$, leg. Schillhammer et al. (120), sifting of dry and moist leaf litter along stream and in nearby forest (1, NHMW); env. Maymyo, 700-900 m, 12-13.II.1996, leg. S. Kurbatov (2, MHNG), same but 4-5.III.1996 (1, MHNG); Yangon Division, Yangon, Insein, Highland Lodge (Pyay Road, 7.5 miles), 11-12.V.1999, leg. H. Schillhammer \& R. Schuh (38), at light (4, NHMW); Pegu, 60 km NW Yangoon, $17^{\circ} 19^{\prime} \mathrm{N}, 96^{\circ} 28^{\prime} \mathrm{E}, 22 . X \mathrm{XI} 2003$, leg. M. Hornburg, light trap (1, coll. Schülke); NEPAL: Seti (province), Bajhang (district), 12 km NE Chainpur, Talkot Gad S of Talkot, $29^{\circ} 36^{\prime} 17^{\prime \prime} \mathrm{N}, 81^{\circ} 17^{\prime} 54^{\prime \prime} \mathrm{E}, 1400 \mathrm{~m}, 28 . \mathrm{VI} .2009$, leg. A. Weigel (37), riverside, at light (1,

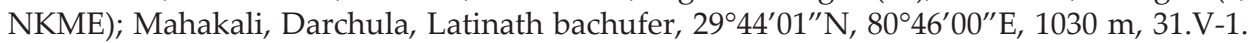
VI.2005, leg. A. Weigel (3, NKME); prov. Bagmati, Dobate Ridge NE Barahbise, 2800 m, 2.V.1981, leg. I. Löbl \& A. Smetana (55d), tamisage de bois pourri, de feuilles mortes et de mousses dans une chênaie avec rhododendrons (1, MHNG); prov. Bagmati, 4 km S Tarang Marang, 900 m, 28.IV.1981, leg. I. Löbl \& A. Smetana (50), tamisage d'herbe et de feuilles mortes près d'une source (2, MHNG); prov. Bagmati, Gokana Forest nr. Kathmandu, 1400 m, 31.III.1981, leg. I. Löbl \& A. Smetana (1b), tamisage dans la forêt (2, MHNG); prov. Bagmati, Gokana Forest nr. Kathmandu, 1400 m, 1.IV.1981, leg. I. Löbl \& A. Smetana (2b), tamisage, dans un ravin boisé, de mousses et de branches mortes (2, MHNG); prov. Bagmati, Nagarjun Forest W Kathmandu, 1650 m, 2.IV.1981, leg. I. Löbl \& A. Smetana (3), forêt secondaire jeune, sur le versant ouest, tamisage de mousses et feuilles mortes (1, MHNG); distr. Kathmandu, Gokarna Forest Reserve, 1350 m, 20.X.1983, leg. I. Löbl \& A. Smetana (40), tamisage de feuilles mortes dans la forêt assez sèche (1, MHNG); distr. Kathmandu, Godawari, 1600 m, forêt au-dessus du jardin botanique, 31.III.1984, leg. I. Löbl (1), tamisage de feuilles mortes près d'un ruisseau, humide (6, MHNG); MALAYSIA: W Ipoh, $5 \mathrm{~km}$ of Tanjong Rambutan, 13-15.IV.2000, leg. M. Snižek (1, coll. Schülke); MALAYSIA/BORNEO: Sabah, Sepilok Forest Res. (near Sandakan), 8.VI.1968, leg. R.W. Taylor, ex humus (1, MHNG); Sabah, Kinabalu Park, Poring Hot Springs, ca. 500 m, forêt à Dipterocarpaceae relativement sèche, 6.V.1987, leg. D. Burckhardt \& I. Löbl (14a), tamisage de bois pourri avec champignons a la lisière de la forêt, et de feuilles mortes au pied des arbres la forêt (4, MHNG); Sabah, Mt. Kinabalu National Park, Poring Hot Springs 480 m, 10.V.1987, leg. A. Smetana (B39), sifting of leaf litter, debris and fermenting fruits on forest floor (2, FMNH); Sabah, Mt. Kinabalu National Park, Poring Hot Springs, 485 m, 25.VIII.1988, leg. A. Smetana (B147), sifting of accumulated old fruits of Areca palms, including debris and humus underneath, open area (1, FMNH), same but 29.VIII.1988 (B160) (2, FMNH); Sabah, Bingkor N Keningau, 400-500 m, 19-20.XI.1996, leg. W. Schawaller (1, SMNS); Sabah, Poring Hot Springs, 450-600 m, 9-11.III.2007, leg. W. Schawaller (3, SMNS); Sarawak, Gn. Gading N. P., 150-300 m, 9-12.III.2008, leg. W. Schawaller (1, SMNS); PHILIPPINES/MINDANAO: 
Davao prov., 25 km NW New Bataan, 1200 m, 20-22.V.1996, leg. Bolm (1, SMNS); PHILIPPINES/LEYTE: Visca N Baybay, 200-500 m, 22.II.1991, leg. W. Schawaller et al., primary forest (3, SMNS), same but 22.II.-10.III.1991 (4, SMNS); Visca N Baybay, 3.III.1991, leg. W. Schawaller et al., cultivated land (1, SMNS); PHILIPPINES/LUZON: Lagunas (Calabarzon)., Mt. Makiling, summit road (S of Los Banos), 500 m, 20.XI.1995, leg. I. Löbl (2), degraded rainforest, bamboo leaf litter and other debris near road (14, MHNG); Lagunas (Calabarzon)., Mt. Makiling, above Mad Springs, 400-700 m, 19-22.XI.1995, leg. J. Kodada, degraded rainforest (5, MHNG); Lagunas (Calabarzon), Mt. Makiling, summit road (S of Los Banos), 600 m, 21-22.XI.1995, leg. I. Löbl (3), degraded rainforest, moss, epiphytes and rotten wood on large fallen trees (1, MHNG); Lagunas (Calabarzon), Mt. Banahaw above Kinabuhayan, 600-700 m, trail to Crystalino, 24.XI.1995, leg. I. Löbl (5a), degraded rainforest, forest floor litter (1, MHNG), same but leg. J. Kodada \& B. Rigová (1, MHNG); Lagunas (Calabarzon), Mt. Banahaw near school about 1 km Kinabuhayan, 500 m, 26.XI.1995, leg. I. Löbl (7a), degraded rainforest, forest floor litter (5, MHNG), same but leg. J. Kodada (4, MHNG); Central Luzon, Mt. Banahaw above Kinabuhayan, summit trail, ca. 800 m, 25. XI.1995, leg. I. Löbl (6), degraded rainforest, on slope (3, MHNG); PHILIPPINES/PALA-

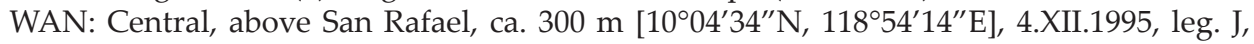
Kodada, degraded rainforest, on slope (4, MHNG), same but leg. I. Löbl (13), leaf litter (9, MHNG); Central, along Tarabanan river NE San Rafael, ca. 30 m, 7.XII.1995, leg. J. Kodada \& B. Rigová (1, MHNG); Central, Concepcion, large logs across Concepcion river NE San

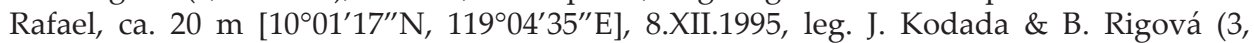
MHNG); INDONESIA/SUMATRA: Jambi, Mt. Kerinci, 1750-1850 m, 11-12.XI.1989, leg. I. Löbl, D. Agosti, D. Burckhardt (11), sifting of vegetational debris in montane LithocarpusCastanopsis forest (1, MHNG); INDONESIA/SULAWESI: Maros, Karaenta Nature Reserve, 500 m, 18.IV.1991, leg. D. Agosti (22) (F91791), limestone, rainforest, clay, thin leaf litter layer and hand collecting (3, MHNG); Pattanetegang, $1600 \mathrm{~m}\left(\right.$ ? , ca. 5²5'S, $\left.119^{\circ} 59^{\prime} \mathrm{E}\right)$, 9.IV.1991, leg. D. Agosti (15), secondary forest, leaf litter layer thin, soil clay, few mycorrhiza (11, MHNG); Ulumambi (W of Mamasa), 1000 m, 11.IV.1991, leg. D. Agosti (F91741), remnants of rainforest / coffee plantation, leaf litter (3, MHNG); Larompang - Temboe (S of Palopo), 50 m, Nature reserve, secondary lowland rainforest, 20.IV.1991, leg. D. Agosti (24), leaf litter layer, thick and heavily infested with mycorrhiza, leaf litter sample and hand collecting (1, MHNG); SE-, near Kolaka, 300 m, 20.V.1997, leg. S. Kurbatov (1, MHNG); INDONESIA/BALI: Penelokan, 25.XI.1978, leg. J. T. Huber (2, MHNG); INDONESIA/ JAVA: West Java, Bogor, Parung, Churuk, 17.VI.-15.VII.2005, leg. S. K. Alekseyev (5, coll. Ryvkin); INDONESIA/SUMBAWA: NE-, Calabai (Tambona N. P.), 11-13.II.1994, leg. Bolm (16, SMNS, 2, HNHM); INDONESIA/LOMBOK: Senaro, N slope of Rinjani, 1100 m, 2-5. II.1994, leg. Bolm (2, SMNS); Suranadi, park with secondary forest, 190 m, 9.III.1991, leg. D. Agosti (1) (F91507), soil with clay, distinct leaf litter layer (4, MHNG); INDONESIA/TIMOR: East Nusa Tengarra prov., between Soe and Kapan, 16 km N Soe, 1000 m, 30.III.1991, leg. D. Agosti (13) (F91654), evergreen forest on limestone, tall trees with butresses and epiphytes, on slope very thin leaf litter layer, after rain, winkler (18, MHNG), same but 29.III.1991 (F91643) (3, MHNG); INDONESIA/MOLUCCAS: Tanimbar, Yamdena isl., Bomaki (NW of Saumlaki), 50 m, 18.IX.1991, leg. D. Agosti (F911146), limestone, Kanary nut forest, evergreen, leaf litter (8, MHNG); INDONESIA/IRIAN JAYA: Sorong prov., Salawatti Isl., Solol, 200-300 m, 6-7.XI.1996, leg. A. Riedel, sifted (6, NHMW); Sorong, Salawatti Isl., Kalobo, 10-30 m, 19-22.X.1996, leg. A. Riedel, sifted (1, NHMW); Sorong prov., Batanta Isl., Waylebet, 500 m, 29.X.1996, leg. A. Riedel, sifted (2, NHMW), same but $800 \mathrm{~m}$ (1, NHMW); Sorong prov., Batanta Isl., Waylebet, 10 m, 30.X.1996, leg. A. Riedel, sifted (8, 
NHMW); Sorong prov., Batanta Isl., Waylebet, 150 m, 2.XI.1996, leg. A. Riedel, sifted (17, NHMW); Sorong prov., Waigeo Isl., Kabui Bay, Wawiay, 50 m, 14.XI.1996, leg. A. Riedel (4, NHMW); Sorong, Intimpura logging camp, dusun Meibo, 100-150 m [00 50'45"S, $\left.131^{\circ} 23^{\prime} 03^{\prime \prime} \mathrm{E}\right]$, 19.I.2001, leg. A. Riedel, sifted in forest (14, SMNS); Wandammen Bay, Wondiwoi Mts., Wasior, 500-700 m [02 $\left.{ }^{\circ} 43^{\prime} 14^{\prime \prime S}, 134^{\circ} 32^{\prime} 41^{\prime \prime} \mathrm{E}\right]$, 14.I.2001, leg. A. Riedel, sifted in forest (1, SMNS); Japen Isl., E Serui, Wawuti, 300 m [01 $50^{\prime} 47^{\prime \prime}$ S, 136 $24^{\prime} 50^{\prime \prime}$ E], 17.XII.2000, leg. A. Riedel, sifted in forest (7, SMNS); Japen Isl., on road to Ambaidiru, Kontiunai, 600$700 \mathrm{~m}$ [01 $\left.47.5^{\prime} \mathrm{S}, 1^{\circ} 6^{\circ} 18.0^{\prime} \mathrm{E}\right], 26 . X \mathrm{XI} .2000$, leg. A. Riedel, sifted in forest (4, SMNS); Jayapura prov., Rouffaer Mts., Mamberamo, Noiadi, 150-200 m, 17.III.1999, leg. A. Riedel, sifted (3, NHMW); PAPUA NEW GUINEA: nr. Lae, ca. 50 m, 11.VI.1972, leg. R.W. Taylor (ANIC Berlesate 391), rainforest (1, ANIC); Passam, near Wewak, ca. 270 m, 5.VII.1972, leg. R.W. Taylor (1, ANIC); near Kokoda, ca. 500 m, 1.VI.1972, leg. R.W. Taylor (4, ANIC); 8 km S of Kokoda, 800 m, 1.VI.1972, leg. R.W. Taylor (3, ANIC); Sandaun prov., N Mianmin, $1100 \mathrm{~m}$

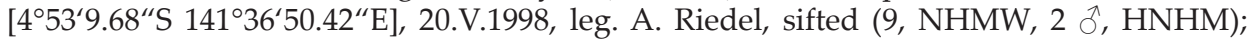
West Sepik prov., Vanimo, Denake-range, km 12, $500 \mathrm{~m}$ [ $\left.2^{\circ} 44^{\prime} 58^{\prime \prime} \mathrm{S}, 1^{\circ} 141^{\circ} 14^{\prime} 54^{\prime \prime} \mathrm{E}\right], 28-$ 29.X.1992, leg. A. Riedel (25B), roadside forest, sifting (6, SMNS); Madang prov., centre of Baiteta forest $\left(05^{\circ} 01^{\prime} \mathrm{S}, 1^{\circ} 5^{\circ} 45^{\prime} \mathrm{E}\right), 1 . \mathrm{V} .1996$, leg. O. Missa (AR41), at light (7, ISNB), same but 21.V.1996 (AR10) (2, ISNB), same but 24.VI.1996 (T2) (2, ISNB), same but 20.VI.1996 (T2) (3, ISNB), same but 15.V.1996 (T11) (2, ISNB); [Madang prov.,] env. Madang, Nagada [Mission] [509.5'S, $145^{\circ} 47.3^{\prime} \mathrm{E}$ ], VI.1979, leg. W.G. Ullrich (3, MHNG); Morobe prov., Mindik Pindiu, near Kuat river, 500-580 m, 28.IV.1998, leg. A. Riedel, sifted (5, NHMW); [Morobe prov.,] Finschhafen, Heldsbach [6²9.5'S, 147050.0'E], XI.1979, leg. W.G. Ullrich (3, MHNG); Morobe prov., Wau region, Wau Ecology Institute (WEI) area, 1400 m, 16.V.1992, leg. G. Cuccodoro (1a), lower montane rain forest, vegetational debris (34, MHNG); Morobe prov., Wau region, WEI area, $1150 \mathrm{~m}, 17 . V .1992$, leg. G. Cuccodoro (2b), very degraded lower montane rain forest, vegetational debris at foot of isolated Klinkii pines - 80\% its leaves (10, MHNG), same but (2c), vegetational debris on rocks in small gallery forest (22, MHNG); Morobe prov., Wau region, WEI area, 1450 m, 21.V.1992, leg. G. Cuccodoro (5b), lower montane rain forest, rotting trunk and vegetational debris (20, MHNG); Morobe prov., Wau region, Mt. Kaindi road, 1350 m, 24.V.1992, leg. G. Cuccodoro (7), garden bordering lower montane rain forest, vegetational debris and rotting trunks (106, MHNG); Morobe prov., Wau region, Mt. Kaindi road, 1700 m, 25.V.1992, leg. G. Cuccodoro (8a), degraded lower montane rain forest, vegetational debris and rotting trunks (1, MHNG); Morobe prov., Wau region, WEI area, $1200 \mathrm{~m}, 26 . V .1992$, leg. G. Cuccodoro (9a), coffee plantation, rotting wood (2, MHNG), same but (9b), vegetational debris (1, MHNG), same but (9c), bark and basidiomycetes (2, MHNG); Morobe prov., Wau region, WEI area, $1150 \mathrm{~m}$, 27.V.1992, leg. G. Cuccodoro (10b), very degraded lower montane rain forest, rotting trunk (4, MHNG), same but (10c), basidiomycetes (1, MHNG); Morobe prov., Wau region, Bieru road (Kaisenik school), 1050 m, 28.V.1992, leg. G. Cuccodoro (11c), gallery forest, moss and vegetational debris (2, MHNG), same but (11d), vegetational debris (7, MHNG).

Redescription - Measurements (in mm, $\mathrm{n}=10)$ : $\mathrm{HW}=0.55(0.52-0.58)$; $\mathrm{TW}=0.51(0.47-$ $0.54) ; \mathrm{PW}=0.72(0.67-0.78) ; \mathrm{EW}=0.82(0.73-0.92) ; \mathrm{AW}=0.81(0.74-0.92) ; \mathrm{HL}=0.43$ (0.39$0.46) ; \mathrm{EL}=0.15(0.14-0.16) ; \mathrm{TL}=0.13(0.12-0.14) ; \mathrm{PL}=0.44(0.42-0.47) ; \mathrm{SC}=0.53(0.47-0.60)$; $\mathrm{FB}=1.44$ (1.34-1.55); BL = 2.62 (2.40-2.88). Habitus as in Fig. 94. Forebody moderately shining despite sculpture, abdomen very finely microsculptured and with large but extremly shallow, obscured punctures, less lustrous. Elytra reddish dark brown to sometimes blackish, head, pronotum, abdomen and legs medium to dark brown, except explanate part of pronotum and transparent clypeal area lighter, mouthparts and antennomeres 1-4 


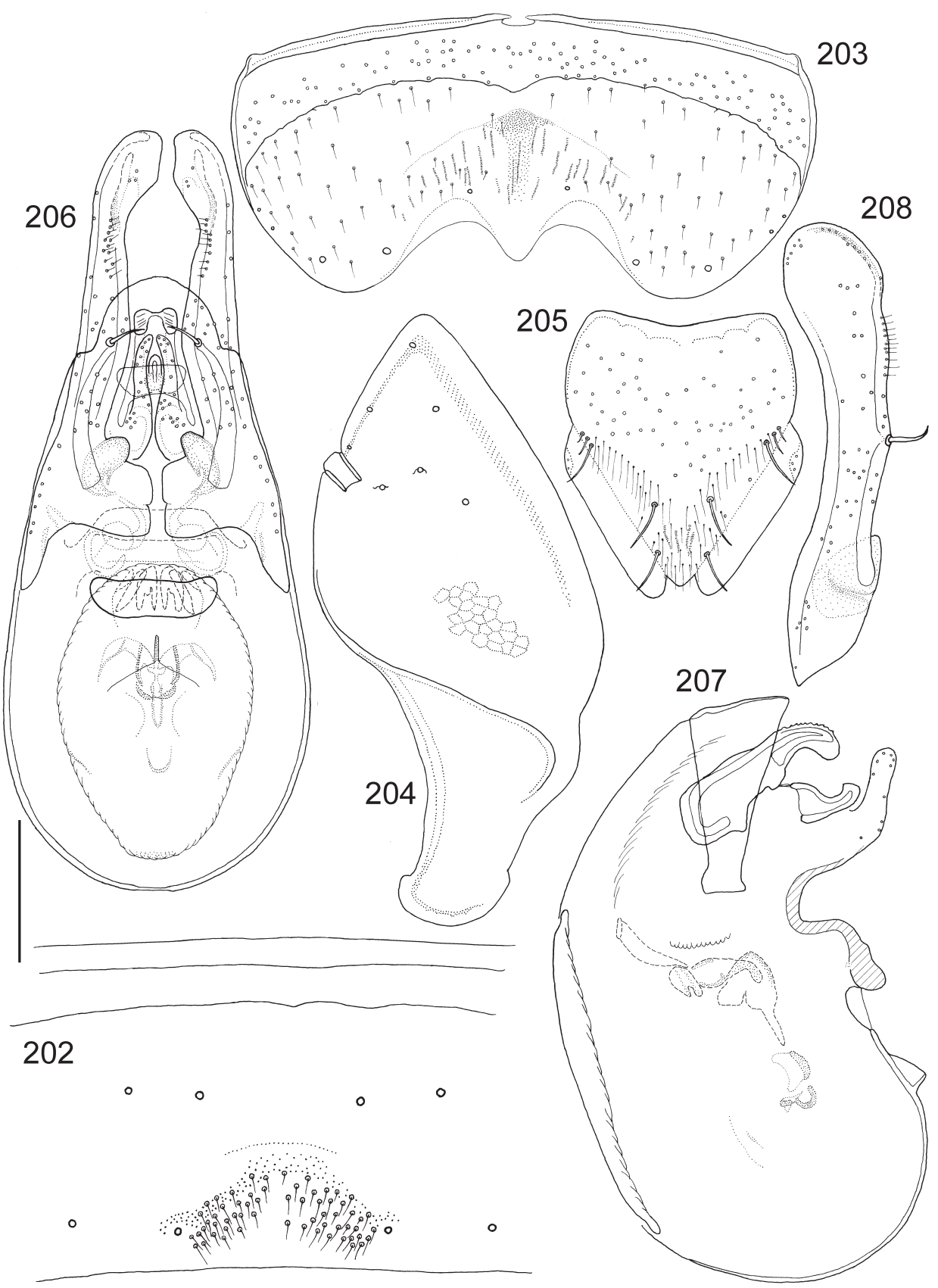

Figs 202-208. Anotylus pseudopsinus (Fauvel, 1895), male. 202 = median part of sternite VII, 203 = sternite VIII, 204 = tergite IX, 205 = tergite X, 206 = aedeagus, frontal view, $207=$ median lobe, lateral view, 208 = paramere, lateral view. Scales: $0.10 \mathrm{~mm}$ for Figs 206-208, 0.115 mm for Figs 202, 204-205, 0.125 mm for Fig. 203 
medium brown, other antennomeres dark brown. Head with an extremely thin rim on anterior margin, latter rather truncate in middle, slightly angled also at supraantennal prominences, these as moderately elevated oblique ridges, disc slightly impressed near eyes. Epistomal suture forming subcircular impression (less punctured inside), upper surface without tactile setae, eyes moderately large and somewhat bulging from sideline of head; in dorsal view temples (less than length of eye or slightly more in case of brachypter morph) alatiform, laterally rounded and posteriorly constricted to form distinct neck, however, dorsally not separated by occipital furrow, only by dorsum of neck being microsculptured rather than punctured. Antennomere $1 \mathrm{club}$-shaped and very lightly microsculptured, segment 2 basally broad oval, segment 3 stout club-shaped, segment 4 slightly transverse discoid, segments 5-11 with basal dishes, segments 6-10 transverse (about 1.5× broader than long). Head and pronotum with mostly alveolate sculpture. Pronotum with lateral portion explanate and slightly up-turned, edge alatiform with sparse short setae; lateral margin unevenly arched with a number of small, irregularly distributed denticles, posterior margin broadly and rather shallowly incised before corners; disc medially with two nearly parallel longitudinal ridges (posteriorly confluent or almost), impressed alongside them, laterally impressed around middle of sides. Elytra with very thin membranous posterior margin extending from sutural corner to $4 / 5$ of hind margin. Elytral epipleural ridge present with slightly up-turned lateral alatiform portions, disc longitudinally weakly impressed alongside; shoulders well developed, surface foveolate, in between punctures torulose. Legs short, pro- and mesotibia with several spinulose rows, metatibia with longitudinal ctenidium of spinules in distal half. Abdomen with sides rather arcuate, second segment with paratergites strongly broadening posteriorly (abdomen appearing constricted at base), those of segments III-VII thin, mesal paratergites broad; posterior edge of tergite VII with thin palisade fringe, median part of sternite VII as in Fig. 202, sternite VIII (Fig. 203), tergites IX (Fig. 204), X (Fig. 205) and aedeagus as in Figs 206-208.

Distribution - The species is widely distributed from the whole range of the Himalaya to southern China and Vietnam to the Philippines, the Greater Sunda Islands and New Guinea (see also below).

Remarks - The holotype of Oxytelopsis cassagnaui Coiffait, 1982 is lost (similarly to that of $O$. franzi described in the same article). While most of the synonyms of this name seem to represent forms well within the (great) range of variation known for this species, some remarks must be made as regards 'brevipennis Bernhauer'. Apart from different body proportions, examination of male genitalia and terminalia only revealed a few subtle differences from the mainland SE Asian forms; these deviations are too small to allow for safe recognition of a distinct taxon, so it is here kept within $A$. pseudopsinus. This form with smaller elytra and comparatively smaller eyes and longer temples exists mostly east of Wallace's Line. There is still a great range of variation existing within those southeastern populations, with rather different external appearances with genitalia quite uniform. To confuse matters even more, a similar population with smaller elytra exists on the island of Ceylon (Sri Lan$\mathrm{ka}$ ). The description and genitalia illustrations provided here are based on the mainland SE Asian form (cf. the type of 'pseudopsinus'), but the habitus pho- 
tograph is of a specimen with smaller elytra (cf. the type of 'brevipennis'). The name 'brevipennis' is a younger secondary homonym and must be replaced if used as valid.

\author{
Anotylus reitteri (Bernhauer, 1934)
}

(Figs 79, 209-215)

Oxytelopsis reitteri Bernhauer, 1934: 1; Herman 1970: 413; Herman 2001: 1414.

Examined type material - Oxytelopsis reitteri Bernhauer - Holotype (†): "Hwa-YinShan; MittelSzechuan; 1600 m, Reitter \Oxytelopsis; Reitteri Brh; Typus \Reitteri Brnh; Typus unic.; Oxytelopsis \Reitteri Bernh.; Ent. Nachr.bl. 1934,; p. $1 \backslash$ Holo-; type [red margined disc, curator label] \Oxytelopsis; reitteri Bnh; P.M. Hammond; det. 1974; Holotype q \FMNH-INS; $0000063475 \backslash$ Photographed; D. Le 2011; EMu catalog" (FMNH).

Other material - CHINA: Jiangxi prov., Jinggangshan, 700-900 m, 26.V.-02.VI.1998, leg. S. A. Kurbatov (1 ô, 1 +, NHMW, 1 ô, MHNG); Sichuan, Middle-, Hwa-Yin-Shan, 1600 $\mathrm{m}$, leg. E. Reitter $(1+\mathrm{q}, \mathrm{BMNH}$, marked as type but is not).

Redescription - Measurements (in mm, n=5): HW $=0.65(0.63-0.67) ; \mathrm{TW}=0.64(0.63-$ $0.66) ; \mathrm{PW}=0.88$ (0.85-0.89); $\mathrm{EW}=0.97$ (0.93-1.00); $\mathrm{AW}=1.03(1.00-1.08) ; \mathrm{HL}=0.50(0.48-$ $0.51) ; \mathrm{EL}=0.13(0.12-0.14) ; \mathrm{TL}=0.21(0.20-0.22) ; \mathrm{PL}=0.55(0.54-0.56) ; \mathrm{SC}=0.60(0.57-0.62)$; $\mathrm{FB}=1.75$ (1.71-1.78); $\mathrm{BL}=3.48$ (3.38-3.61). Habitus as in Fig. 79. Forebody weakly shining because of sculpture, abdomen very finely microsculptured, finely and loosely punctured, somewhat more lustrous. Body reddish medium to dark brown, head (except clypeal area) often darker, blackish dark brown, middle of tergite bases often blackish as well as most of antennomere 1. Head with anterior margin possessing rim (weaker in middle), evenly arched but slightly angled at supraantennal prominences, these as moderately elevated oblique ridges, rim continuing posteriad along inner border of eyes, disc slightly impressed beside it. Epistomal suture forming circular impression (less sculptured inside), upper surface with weak tactile setae (intratemporalis) situated postero-medially of each eye. Eyes medium-sized and slightly bulging, in dorsal view temples (1.6× length of eye) alatiform, laterally rounded and posteriorly constricted to form distinct neck, separated by transversal, weak occipital furrow. Antennomere 1 flattened club-shaped and strongly microsculptured, segment 2 oval but broad at base, segment 3 club-shaped, segment 4 small and slightly transverse, segments 5-11 with basal dishes, articles 6-10 transverse (about $1.5 \times$ broader than long), apex of segment 11 lighter. Head with areolate-rugose, pronotum with lacunose sculpture. Pronotum with lateral portion explanate and slightly up-turned, edge alatiform with very sparse short setae; lateral border unevenly arched with thin marginal ridge and a number of small, irregularly distributed denticles, posterior margin evenly arched; disc medially with two nearly parallel and posteriorly confluent longitudinal ridges, posteriorly impressed alongside them, laterally somewhat impressed towards side margin. Elytra with membranous posterior margin extending from sutural corner to 3/4 of hind margin. Elytral epipleural ridge apparent as stronger sculpture lines in lateral alatiform portions, disc with very slight to no impression in centre; shoulders well developed, surface obscurely foveolate and in between punctures slightly torulose. Legs short, pro- and mesotibia with several spinulose rows, metatibia with longitudinal ctenidium of spinules in distal half. Abdomen with sides moderately arcuate, second seg- 


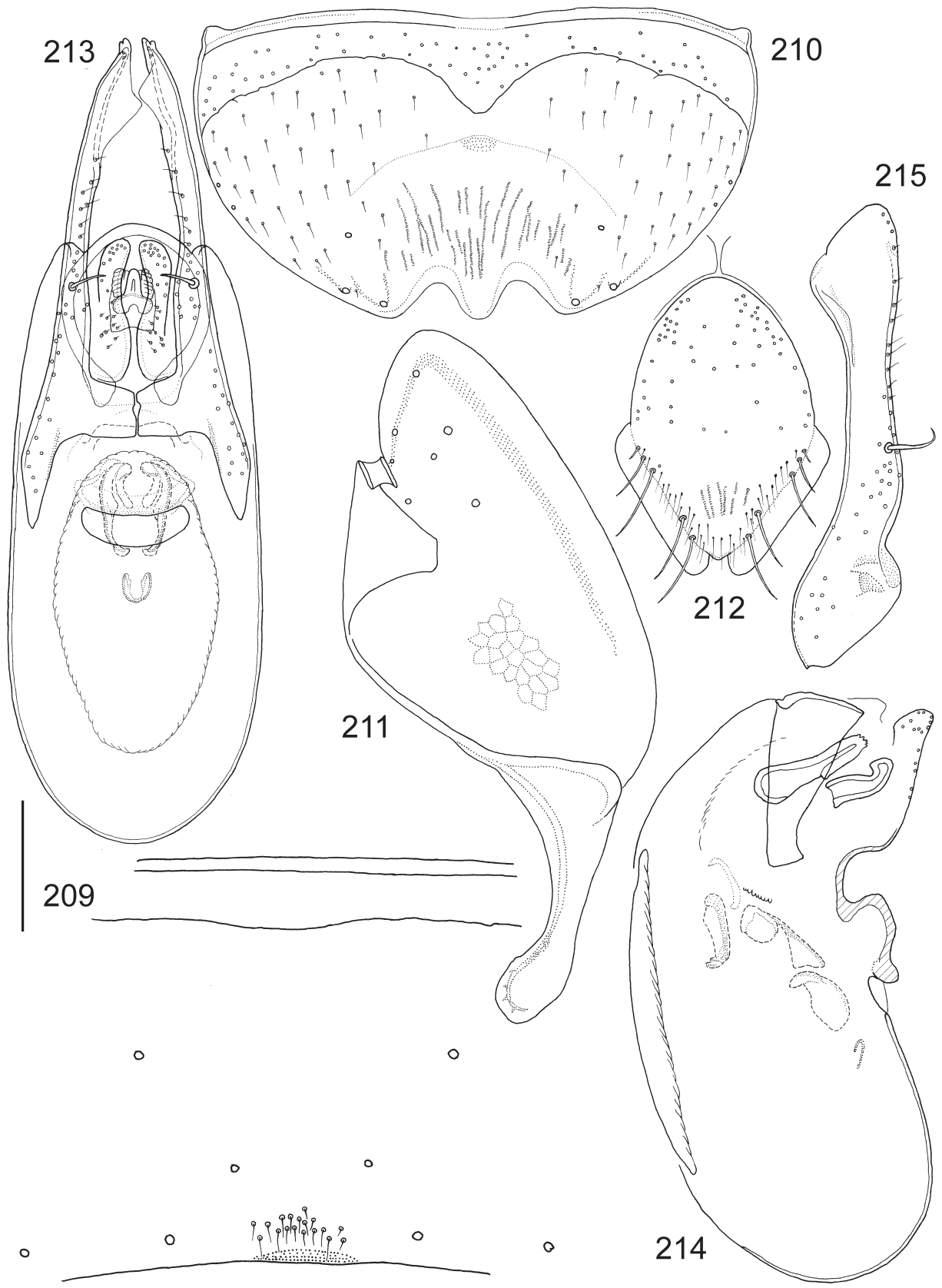

Figs 209-215. Anotylus reitteri (Bernhauer, 1934), male. $209=$ median part of sternite VII, 210 = sternite VIII, 211 = tergite IX, $212=$ tergite $X, 213=$ aedeagus, frontal view, $214=$ median lobe, lateral view, 215 = paramere, lateral view. Scales: $0.10 \mathrm{~mm}$ for Figs $213-215,0.11$ mm for Figs 209, 211-212, 0.14 mm for Fig. 210 
ment with paratergites broadening posteriorly (abdomen slightly constricted at base), those of segments III-VII thin, mesal paratergites broad; posterior edge of tergite VII with thin palisade fringe, median part of sternite VII as in Fig. 209, sternite VIII (Fig. 210), tergites IX (Fig. 211), X (Fig. 212) and aedeagus as in Figs 213-215.

Distribution - The species is only known from the Chinese provinces of Sichuan and Jiangxi.

Remarks - Considering the problematic identification of this taxon (original material is only two females) and the poor condition of the subsequently identified specimens, more work is needed before a better separation can be made from closely related congeners.

\section{Anotylus riedeli sp. $\mathrm{n}$.}

(Figs 91, 216-222, 272)

Type material - Holotype ( $\left.\delta^{\Uparrow}\right)$ : INDONESIA/JAVA: W-, [Bandung,] Rancabali, W

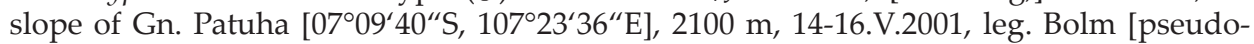
nym of Czech couple, collectors who chose to withhold their identities] (SMNS); Paratypes (122): same data as holotype, (2 2 , SMNS); INDONESIA/JAVA: W-, "Ranca Upas", 10 km S Ciwidey, ca. 1300 m, 9.VIII.1994, leg. R. Schuh, forest litter (2 §, 1 क, NHMW, 1 ઈ, HNHM); W-, Garut, Cilawu, Gn. Cikuray, 07¹8’34.7"S, 10752'37.3”E, 1648 m, 24.IX.2005,

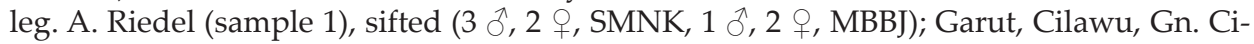
kuray, $07^{\circ} 18^{\prime} 50.4^{\prime \prime} \mathrm{S}, 107^{\circ} 52^{\prime} 30.7^{\prime \prime} \mathrm{E}, 1800 \mathrm{~m}$, 24.IX.2005, leg. A. Riedel (sample 2), sifted (5

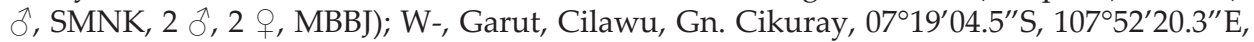
2050 m, 24.IX.2005, leg. A. Riedel (sample 3), sifted (14 §, 10 q, SMNK, 1 ठ, 2 q, MBBJ, 7 , , SMNS, $1 \hat{\jmath}$, ZMUC, $1 \hat{\jmath}, 1$ ㅇ, MHNG, $1 \hat{\jmath}, \mathrm{ZMHB}, 1 \hat{\jmath}, \mathrm{BMNH}, 1 \hat{\jmath}, \mathrm{AMNH}, 1 \hat{\jmath}, \mathrm{CNCI}, 1$

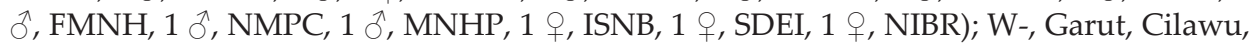

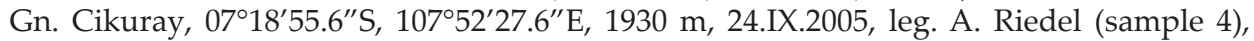

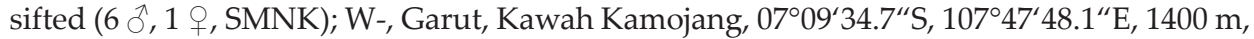
26.IX.2005, leg. A. Riedel (sample 2), sifted (3 $\hat{0}, 7$, SMNK, $1 \hat{0}, 4$, , MBBJ); E-, Banyuwangi, Gn. Ijen, Licin, $08^{\circ} 05.603^{\prime} \mathrm{S}, 114^{\circ} 14.366^{\prime} \mathrm{E}, 1575 \mathrm{~m}, 28$.VIII.2006, leg. A. Riedel (sam-

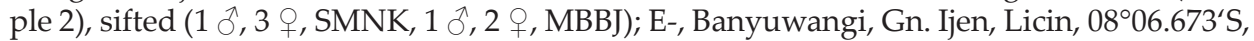

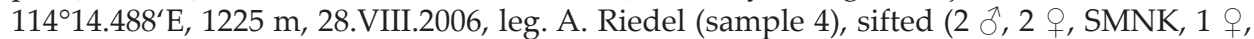
MBBJ); E-, Banyuwangi, Gn. Ijen, Kluncing, $08^{\circ} 07.986^{\prime} \mathrm{S}, 114^{\circ} 11.834^{\prime} \mathrm{E}, 1130 \mathrm{~m}, 30 . \mathrm{VIII} .2006$, leg. A. Riedel (sample 2), sifted (1 ô, 2 +, SMNK, 1 ㅇ, MBBJ); E-, Banyuwangi, Gn. Ijen, Licin, $08^{\circ} 06.673^{\prime} \mathrm{S}, 114^{\circ} 14.488^{\prime} \mathrm{E}, 1225 \mathrm{~m}, 31$.VIII.2006, leg. A. Riedel (sample 4), sifted (3 $\mathrm{O}^{\top}, 4$

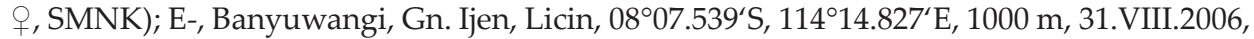
leg. A. Riedel (sample 5), sifted (1 + , SMNK); E-, Banyuwangi, Gn. Ijen, Licin, $08^{\circ} 07.115^{`} \mathrm{~S}$, $114^{\circ} 14.650^{`} \mathrm{E}, 1100 \mathrm{~m}, 31 . \mathrm{VIII} .2006$, leg. A. Riedel (sample 6), sifted $(3 \hat{\jmath}, 1$ ㅇ, SMNK, 1 ๙ , 1 ㅇ, MBBJ).

Description - Measurements (in mm, $\mathrm{n}=10)$ : $\mathrm{HW}=0.77(0.71-0.83) ; \mathrm{TW}=0.78(0.72-$ $0.83) ; \mathrm{PW}=1.18$ (1.06-1.26); $\mathrm{EW}=1.22(1.11-1.33) ; \mathrm{AW}=1.23(1.13-1.31) ; \mathrm{HL}=0.58(0.54-$ $0.62) ; \mathrm{EL}=0.14(0.13-0.15) ; \mathrm{TL}=0.23(0.21-0.25) ; \mathrm{PL}=0.67$ (0.59-0.73); $\mathrm{SC}=0.65$ (0.59-0.71); $\mathrm{FB}=1.96(1.77-2.09) ; \mathrm{BL}=3.67$ (3.35-3.95). Habitus as in Fig. 91. Forebody rather shining despite sculpture, abdomen very finely microsculptured and medium finely, rather shallowly punctate, duller. Forebody blackish dark brown with reddish tint, abdomen and 


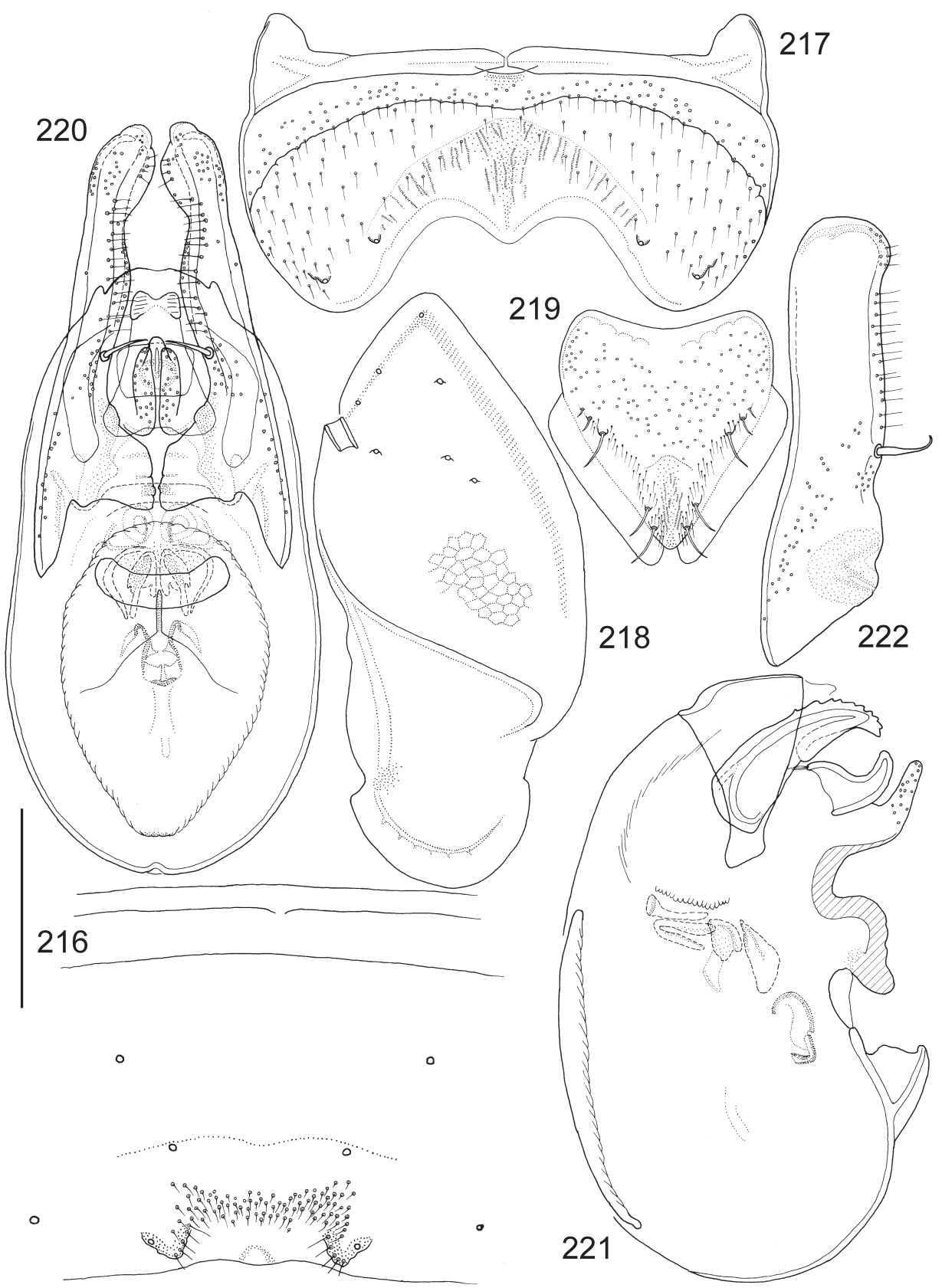

Figs 216-222. Anotylus riedeli sp. n., male. $216=$ median part of sternite VII, $217=$ sternite VIII, $218=$ tergite IX, 219 = tergite $X, 220$ = aedeagus, frontal view , 221 = median lobe, lateral view, 222 = paramere, lateral view. Scales: $0.20 \mathrm{~mm}$ for Figs 216, 220-222, $0.25 \mathrm{~mm}$ for Figs 218-219, $0.28 \mathrm{~mm}$ for Fig. 217 
legs reddish dark brown, mouthparts and antennae reddish medium to dark brown. Head with anterior margin forming an arch truncate in middle, with very slight rim, supraantennal prominences as moderately elevated oblique ridges, disc slightly impressed near eyes, epistomal suture forming subcircular impression (finer and more loose punctation inside) upper surface without tactile setae, eyes rather small and very slightly bulging from sideline of head; in dorsal view temples (1.6× length of eye) alatiform, laterally evenly rounded, posteriorly constricted to form distinct neck, however, dorsal side with only traces of occipital furrow, dorsum of neck demarcated by bearing microsculpture instead of punctation. Antennomere 1 club-shaped, smooth, segment 2 basally broad oval, segment 3 club-shaped, segment 4 small and isodiametrical, segments 5-11 with basal dishes, articles 6-10 transverse (more than 1.5× broader than long), antennomeres 9-10 bulkier than preceding. Head and pronotum with mostly foveate sculpture, varying but mostly very small interspaces. Pronotum with lateral portion mostly explanate and up-turned, edge alatiform with sparse short setae; lateral margin arched and zigzagged with a number of small, irregularly distributed denticles, posterior margin strongly incised in a variable zigzagged fashion before corners; disc medially with two almost parallel longitudinal ridges, impressed alongside them, lateral parts impressed (mostly around middle). Elytra with conspicuous membranous posterior margin extending from sutural corner to $4 / 5$ of hind margin, a small incision before outer corner. Elytral epipleural ridge present with up-turned lateral alatiform portions, disc longitudinally impressed alongside; shoulders well developed, surface foveolate, in between punctures torulose. Legs short, pro- and mesotibia with several spinulose rows, metatibia with longitudinal ctenidium of spinules in distal half. Abdomen with sides moderately arcuate, second segment with paratergites broadening posteriorly (abdomen appearing constricted at base), those of segments III-VII thin, mesal paratergites broad; posterior edge of tergite VII with thin palisade fringe, median part of sternite VII as in Fig. 216, sternite VIII (Fig. 217), tergites IX (Fig. 218), X (Fig. 219) and aedeagus as in Figs 220-222, spermatheca as in Fig. 272.

Etymology - The species is named after the collector of most of the material of this species, Alexander Riedel (SMNK).

Distribution - Only occurs on the island of Java, but apparently in several areas.

Remarks - The taxon is closely related to A. lucidulus and to a lesser extent also to $A$. ijen, both of which are endemic to Java.

\section{Anotylus rurukan sp. n. \\ (Figs 96, 223-229, 273)}

Type material - Holotype (ふ): INDONESIA/SULAWESI: N-, Tomohon, Rurukan,

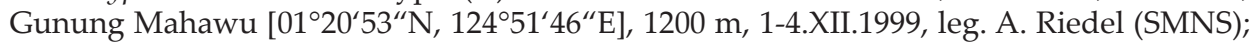
Paratypes (35): same data as holotype (8 $\hat{0}$, SMNS); N-, Tomohon, Rurukan, Gn. Mahawu,

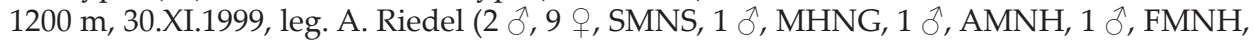
$1 \hat{\jmath}, \mathrm{ZMUC}, 1 \hat{\jmath}, \mathrm{ZMHB}, 1 \hat{\jmath}$, SDEI, $1 \hat{\jmath}$, NMPC, $1 \hat{\jmath}$, NIBR, $1 \hat{\jmath}, \mathrm{BMNH}, 1 \hat{\jmath}, \mathrm{ISNB}, 1 \hat{\jmath}$, MNHP, $1 \hat{\jmath}$, CNCI, $1 \hat{\jmath}$, HNHM); 2 km W Tomohon, bottom of Mt. Lokon, 830-850 m, $01^{\circ} 21^{\prime} 29^{\prime \prime} \mathrm{N}, 124^{\circ} 48^{\prime} 57^{\prime \prime} \mathrm{E}, 29-31 . I .2004$, leg. A Skale (1 +, NHMW); N-, [nr.] Modoinding, Kotamobagu, Gn. Ambang [00 $\left.45^{\prime} 27^{\prime \prime} \mathrm{N}, 124^{\circ} 25^{\prime} 23^{\prime \prime} \mathrm{E}\right], 1450$ m, 6.XII.1999, leg. A. Riedel 
(1 § , SMNS); Umg. Modoinding, NE Kotamobagu, 19.IV.1992, leg. M. Jäch (12), various streams, inside forest and at a forest pool (1, NHMW).

Description - Measurements (in mm, $\mathrm{n}=10)$ : $\mathrm{HW}=0.60(0.53-0.64)$; $\mathrm{TW}=0.57(0.51-$ $0.61) ; \mathrm{PW}=0.86(0.75-0.92) ; \mathrm{EW}=0.90(0.84-0.97) ; \mathrm{AW}=0.96(0.88-1.03) ; \mathrm{HL}=0.47(0.43-$ $0.50) ; \mathrm{EL}=0.12(0.11-0.13) ; \mathrm{TL}=0.18(0.16-0.19) ; \mathrm{PL}=0.48(0.41-0.51) ; \mathrm{SC}=0.54$ (0.49-0.57); $\mathrm{FB}=1.54(1.42-1.62) ; \mathrm{BL}=3.02$ (2.77-3.20). Habitus as in Fig. 96. Forebody weakly shining because of sculpture; abdomen with dense puncation, large (but shallow), apically finer punctures, from tergite VI onwards abdomen very finely coriaceous microsculptured, more lustrous than forebody. Forebody, legs and abdomen reddish dark brown, except explanate part of pronotum and clypeal area (both for transparency), these, mouthparts and antennae reddish medium brown. Head rather transverse (clypeal area less projecting forward), with a thin rim on anterior margin, truncate in middle, very slightly angled also at supraantennal prominences, these as moderately elevated oblique ridges, disc slightly impressed near eyes. Epistomal suture forming subcircular impression (less punctured inside), upper surface without tactile setae, eyes moderately small, very slightly bulging from sideline of head; in dorsal view temples (1.5× length of eye) alatiform, laterally imperfectly rounded (without being angled) and posteriorly constricted to form distinct neck, not separated dorsally by occipital furrow, only by neck being microsculptured rather than punctured. Antennomere 1 club-shaped, finely sculptured, segment 2 elongate oval, segment 3 club-shaped, segment 4 small and very slightly transverse, segments 5-11 with basal dishes, articles 6-10 transverse (about 1.3× broader than long), segments 9-10 bulkier than preceding. Head and pronotum with mostly lacunose sculpture. Pronotum with lateral portion explanate and slightly up-turned, edge alatiform with sparse short setae; lateral margin unevenly arched with a number of small, irregularly distributed denticles, posterior margin broadly incised before corners, disc medially with two parallel longitudinal ridges, impressed alongside them, laterally impressed around middle of sides. Elytra with membranous posterior margin extending from sutural corner to $3 / 4$ of hind margin. Elytral epipleural ridge present with up-turned lateral alatiform portions, disc longitudinally impressed alongside; shoulders well developed, surface foveolate, in between punctures torulose. Legs short, pro- and mesotibia with several spinulose rows, metatibia with longitudinal ctenidium of spinules in distal half. Abdomen with sides rather arcuate, second segment with paratergites strongly broadening posteriorly (abdomen appearing constricted at base), those of segments III-VII thin, mesal paratergites broad, posterior edge of tergite VII with thin palisade fringe, median part of sternite VII as in Fig. 223, sternite VIII (Fig. 224), tergites IX (Fig. 225), X (Fig. 226) and aedeagus as in Figs 227-229, spermatheca as in Fig. 273.

Etymology - The specific epithet is from the type locality, Rurukan (noun in apposition).

Distribution - So far it is only known from northern Sulawesi (Indonesia). Remarks - This species is closely related to A. tanator (also from Sulawesi, but from the southern part). 


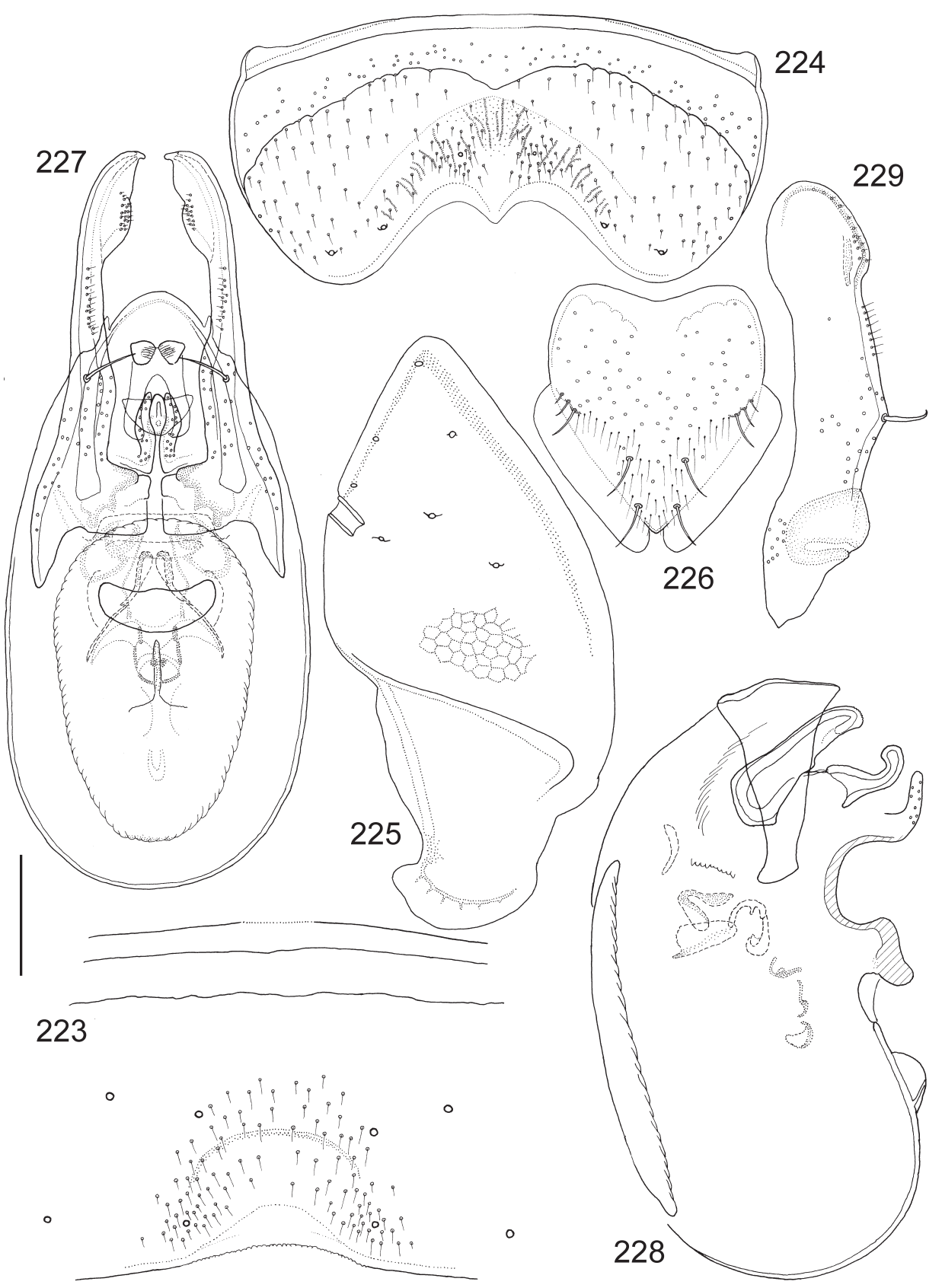

Figs 223-229. Anotylus rurukan sp. n., male. $223=$ median part of sternite VII, $224=$ sternite VIII, 225 = tergite IX, 226 = tergite X, 227 = aedeagus, frontal view, 228 = median lobe, lateral view, 229 = paramere, lateral view. Scales: $0.10 \mathrm{~mm}$ for Figs 226-229, $0.12 \mathrm{~mm}$ for Figs 223, 225, $0.14 \mathrm{~mm}$ for Fig. 224 
Anotylus schawalleri sp. n.

(Figs 73, 230-236, 274)

Type material - Holotype (đ): MALAYSIA/BORNEO: Sabah, Crocker Range N. P., NW Keningau, 900-1200 m [05²6`N, 11605`E], 16-20.XI.1996, leg. W. Schawaller (SMNS); Paratypes (4): same data as holotype (1 $\hat{\delta}, \mathrm{HNHM);} \mathrm{Sabah,} \mathrm{Crocker} \mathrm{Range,} \mathrm{1550-1650} \mathrm{m,}$ à proximité du col (route Kota Kinabalu - Tambunan) [05 $47^{\prime} 55^{\prime \prime} \mathrm{N}, 116^{\circ} 20^{\prime} 06^{\prime \prime} \mathrm{E}$ ], forêt de Lithocarpus-Castanopsis, 16.V.1996, leg. D. Burckhardt \& I. Löbl (27a), tamisage de bois pourri, feuilles mortes et mousses (1 $\delta$, MHNG); Sabah, Crocker Range, face ouest (W), 1600 m env., km 51-52 - route de Kota Kinabalu - Tambunan [0550'04“N, $\left.116^{\circ} 20^{\prime} 08^{\prime \prime} \mathrm{E}\right]$, forét humide à Lithocarpus, Castanopsis et fougères arborescentes, 18.V.1996, leg. D. Burckhardt \& I. Löbl (30a), tamisage de débris vegetaux dans un ravin humide et à la lisière de la forêt (1 +, MHNG); Sabah, Banjaran Crocker Mts., Gunung Alab peak, 30.IV.-27.V.1996, 1650-1800 m [0550`N, $\left.116^{\circ} 20^{`} \mathrm{E}\right]$, leg. M. Štrba \& R. Hergovits (1 o̊ , NHMW).

Description - Measurements (in mm, $\mathrm{n}=5)$ : $\mathrm{HW}=0.60(0.58-0.61) ; \mathrm{TW}=0.57(0.56-0.59)$; $\mathrm{PW}=0.82(0.80-0.85) ; \mathrm{EW}=0.94(0.92-0.96) ; \mathrm{AW}=0.92(0.91-0.93) ; \mathrm{HL}=0.43(0.42-0.44) ; \mathrm{EL}$ $=0.14(0.13-0.15) ; \mathrm{TL}=0.18(0.17-0.19) ; \mathrm{PL}=0.51(0.50-0.52) ; \mathrm{SC}=0.60(0.59-0.61) ; \mathrm{FB}=1.61$ (1.58-1.63); BL = 3.05 (2.91-3.25). Habitus as in Fig. 73. Forebody weakly shining despite sculpture, abdomen with punctures of varying sizes, rather dense but shallow, indistinctly microsculptured, yet more lustrous than forebody. Head blackish dark brown, pronotum, elyra and abdomen reddish dark brown, occasionally lighter, medium brown stripe along posterior elytral margin, lateral pronotal margin and anterior margin of head. Legs, mouthparts and antennae reddish medium brown. Head with anterior margin possessing rather strong rim, almost truncate in front, laterally straight to supraantennal prominences (moderately elevated oblique ridges), disc slightly impressed near eyes. Epistomal suture forming subcircular impression (much less punctate inside), upper surface with weak tactile setae (intratemporalis) situated postero-medially of each eye; eyes medium sized and slightly bulging, in dorsal view temples (1.3× length of eye) alatiform, laterally evenly rounded and posteriorly constricted to form distinct neck separated by weak occipital furrow. Antennomere 1 flattened club-shaped, strongly sculptured, segment 2 basally broad subtriangular, segment 3 incrassate, segment 4 transverse discoid with rim, segments $5-11$ with basal dishes, articles 5-10 transverse (about 1.5-1.7× broader than long). Head and pronotum with mostly alveolate sculpture. Pronotum with lateral portion explanate, edge alatiform with sparse short setae; lateral margin unevenly arched with a number of small, irregularly distributed denticles, posterior corners not incised, disc medially with two weak parallel longitudinal ridges, pronotal sides weakly impressed around middle. Elytra without membranous posterior margin. Elytral epipleural ridge apparent as stronger sculpture lines in lateral alatiform portions, disc with very slight to no impression in centre; shoulders well developed, surface obscurely foveolate and in between punctures torulose. Legs short, proand mesotibia with several spinulose rows, metatibia with longitudinal ctenidium of spinules in distal half. Abdomen with sides moderately/rather arcuate, second segment with paratergites broadening posteriorly (abdomen appearing constricted at base), those of segments III-VII thin, mesal paratergites broad, posterior edge of tergite VII with thin palisade fringe, median part of sternite VII as in Fig. 230, sternite VIII (Fig. 231), tergites IX (Fig. 232), $X$ (Fig. 233) and aedeagus as in Figs 234-236, spermatheca as in Fig. 274.

Etymology - The species is named after the collector of the holotype, Wolfgang Schawaller (SMNS). 


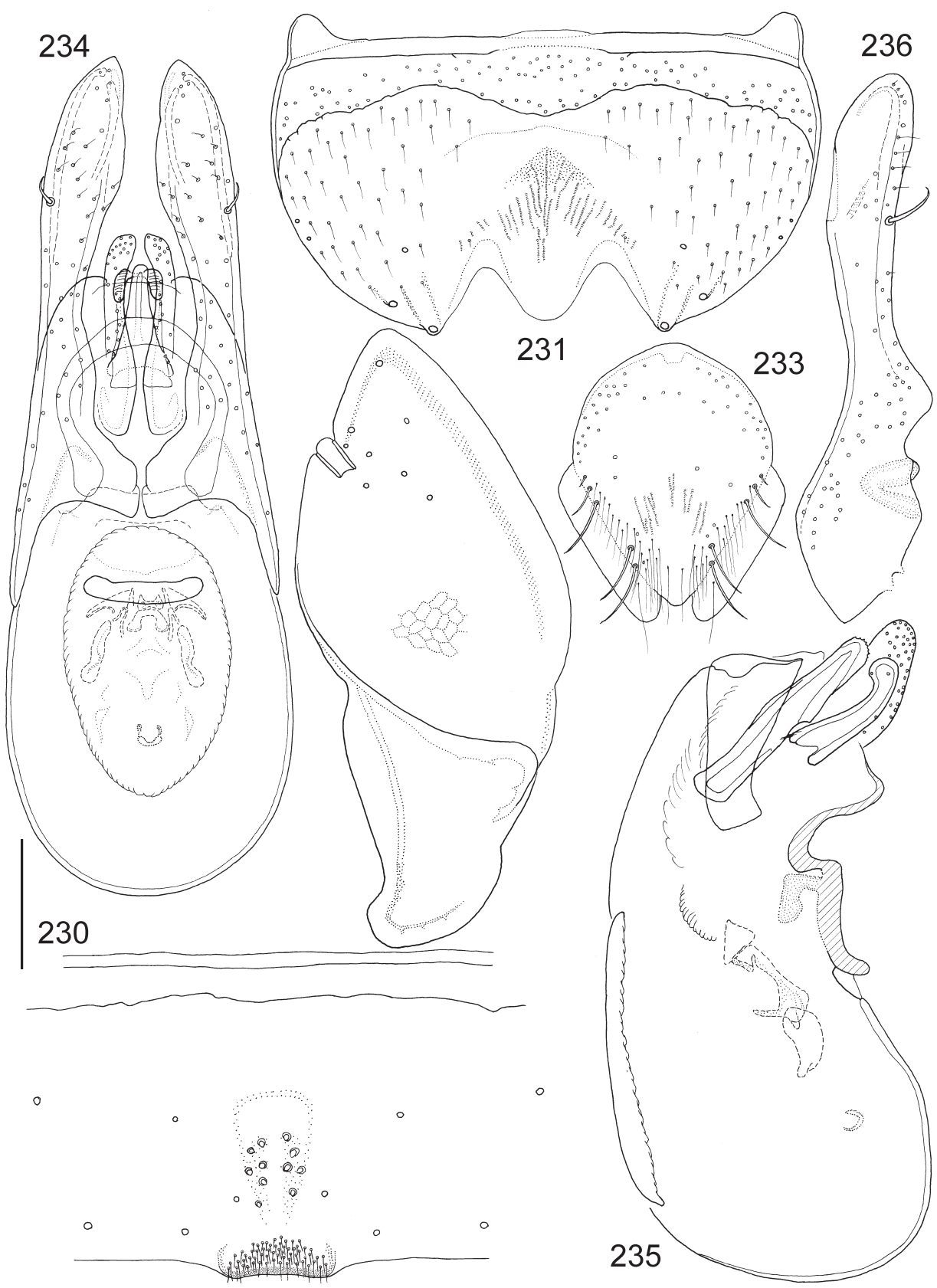

Figs 230-236. Anotylus schawalleri sp. n., male. $230=$ median part of sternite VII, $231=$ sternite VIII, 232 = tergite IX, 233 = tergite X, 234 = aedeagus, frontal view, 235 = median lobe, lateral view, 236 = paramere, lateral view. Scales: $0.10 \mathrm{~mm}$ for Figs 234-236, $0.115 \mathrm{~mm}$ for Fig. 233, $0.15 \mathrm{~mm}$ for Figs 230-232 
Distribution - The species appears to be endemic to the highest areas of the Crocker Range in Sabah (Malaysian Borneo).

Remarks - The low number of specimens examined does not allow a good judgement of intraspecific variability, but externally the species can be difficult to separate from the closely related A. borneensis.

\section{Anotylus schillhammeri sp. n.}

$$
\text { (Figs 83, 237-243, 275) }
$$

Type material - Holotype (đ): MYANMAR: Chin State, WNW Kanpetlet, Natmataung National Park, above base camp, on way to summit, $21^{\circ} 13^{\prime} 21.2^{\prime \prime} \mathrm{N}, 9^{\circ} 55^{\prime} 37.3^{\prime \prime} \mathrm{E}, 2960 \mathrm{~m}$, 8.VI.2010, leg. Aung Zaw Lin (189), remaining patch of degraded moss forest, sifting of leaf

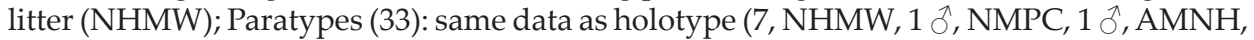

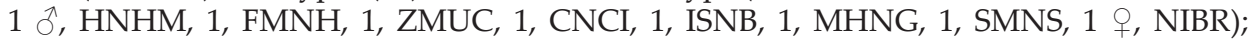
MYANMAR: Chin State, WNW Kanpetlet, Natmataung National Park, $21^{\circ} 13^{\prime} 24.7^{\prime \prime} \mathrm{N}$, $93^{\circ} 58^{\prime} 49.8^{\prime} \mathrm{E}, 2470 \mathrm{~m}, 31 . V .2010$, leg. H. Schillhammer (168), sifting of leaf litter in rather undisturbed forest (2, NHMW); Chin State, WNW Kanpetlet, Natmataung National Park, $21^{\circ} 13^{\prime} 24.7^{\prime \prime} \mathrm{N}, 93^{\circ} 58^{\prime} 49.8^{\prime \prime} \mathrm{E}, 2470 \mathrm{~m}, 2 . \mathrm{VI} .2010$, leg. H. Schillhammer (174), sifting of leaf litter in rather undisturbed forest (4, NHMW); Chin State, WNW Kanpetlet, Natmataung Nat. P., $21^{\circ} 14^{\prime} 03.4^{\prime \prime} \mathrm{N}, 9^{\circ} 59^{\prime} 35.0^{\prime} \mathrm{E}, 2450 \mathrm{~m}, 6 . V I .2010$, leg. H. Schillhammer (185 and 185a), sifting of leaf litter in slightly disturbed forest (10, NHMW).

Description - Measurements (in mm, $\mathrm{n}=10)$ : $\mathrm{HW}=0.71(0.67-0.75)$; $\mathrm{TW}=0.70(0.66-$ $0.74) ; \mathrm{PW}=0.95$ (1.90-1.02); $\mathrm{EW}=1.02$ (0.95-1.09); $\mathrm{AW}=1.10(1.03-1.20) ; \mathrm{HL}=0.55(0.50$ $0.60) ; \mathrm{EL}=0.13(0.12-0.14) ; \mathrm{TL}=0.23(0.22-0.24) ; \mathrm{PL}=0.59$ (0.56-0.62); $\mathrm{SC}=0.42(0.40-0.46)$; $\mathrm{FB}=1.65(1.53-1.76) ; \mathrm{BL}=3.19(2.92-3.62)$. Habitus as in Fig. 83. Forebody moderately shining despite sculpture, abdomen without microsculpture, roughly but not too densely punctate, as shiny as forebody. Head, pronotum, abdomen and antennomeres 5-11 blackish dark brown except supraantennal area and explanate pronotal sides lighter brown (for transparency), elytra, legs, mouthparts and first antennomeres reddish dark brown, antennomeres 2-4 lighter brown. Head with anterior margin possessing thin rim unevenly arched, more or less truncate in middle, also slightly angled at supraantennal prominences (latter as moderately elevated oblique ridges), clypeal rim insignificantly projecting in middle, disc slightly impressed near eyes. Epistomal suture forming circular impression (with only a few tiny punctures inside), upper surface without tactile setae, eyes small and slightly bulging; in dorsal view temples $(1.8 \times$ length of eye) moderately alatiform, laterally rounded and posteriorly constricted to form distinct neck, however, dorsal side not separated by occipital furrow, rather by dorsum of neck bearing microsculpture instead of punctation. Antennomere 1 club-shaped and smooth, segment 2 ovoid, segment 3 clubshaped, segment 4 small and isodiametricalal, segments 5-11 with basal dishes, articles 6-10 slightly transverse (1.0-1.3× broader than long). Head and pronotum with mostly lacunose sculpture. Pronotum with lateral portion explanate and slightly up-turned, edge with sparse short setae, lateral border with several rather prominent angles, gradually transforming into smaller teeth posteriorly, hind margin not incised before corners; disc medially rather convex, parallel longitudinal ridges rather obscured, laterally broadly impressed along sides. Elytra without distinct membranous posterior margin. Elytral epipleural ridge present with up-turned lateral alatiform portions, disc depressed; shoulders 


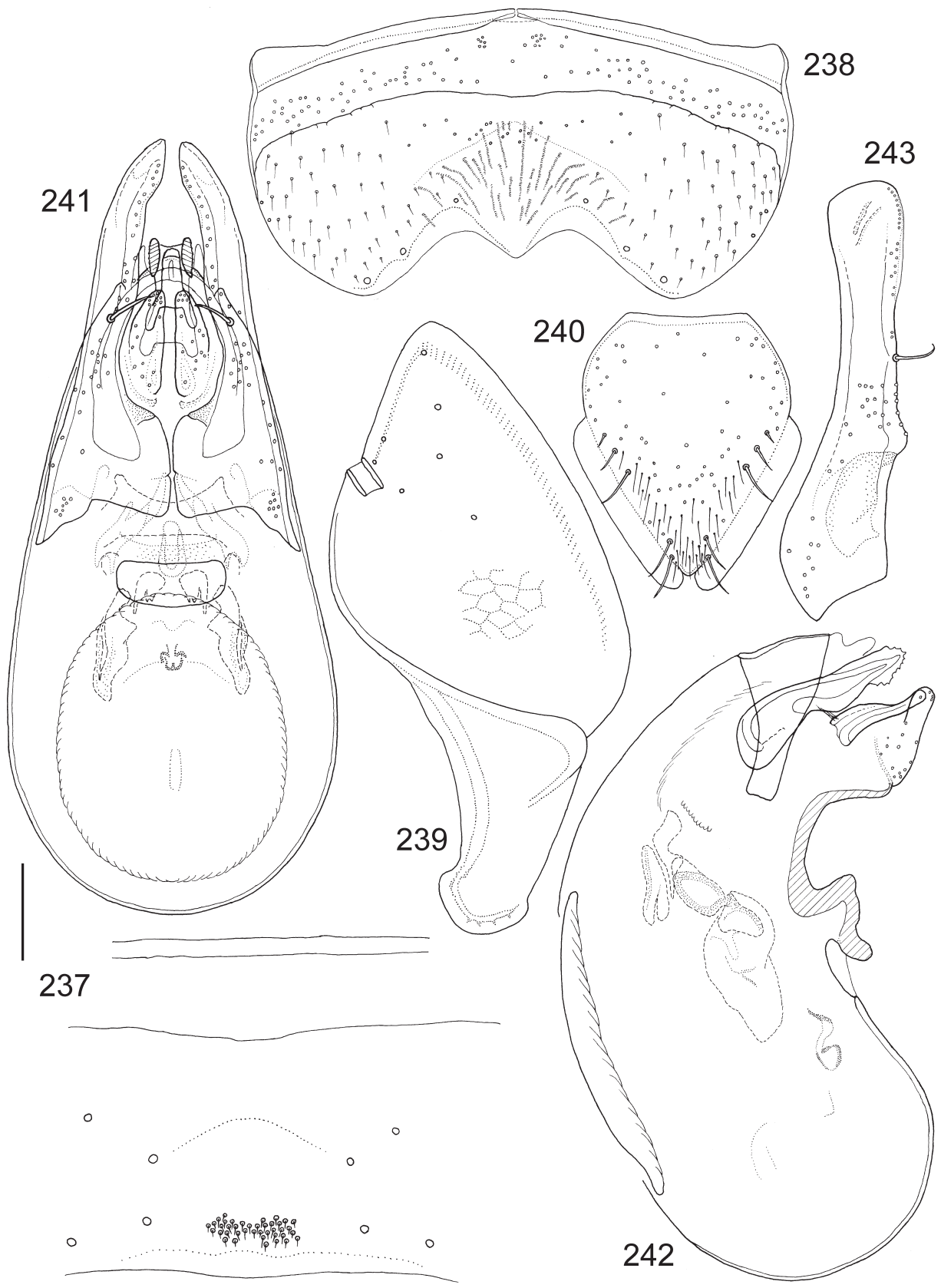

Figs 237-243. Anotylus schillhammeri sp. n., male. $237=$ median part of sternite VII, $238=$ sternite VIII, 239 = tergite IX, 240 = tergite $X, 241$ = aedeagus, frontal view, $242=$ median lobe, lateral view, 243 = paramere, lateral view. Scales: $0.09 \mathrm{~mm}$ for Fig. 237, $0.10 \mathrm{~mm}$ for Figs 241-243, $0.11 \mathrm{~mm}$ for Figs 239-240, $0.12 \mathrm{~mm}$ for Fig. 238 
well developed, surface loosely foveolate, in between punctures mostly shiny. Legs short, pro- and mesotibia with several spinulose rows, metatibia with longitudinal ctenidium of spinules in distal half. Abdomen with sides very weakly arcuate, second segment with paratergites strongly broadening posteriorly (abdomen appearing constricted at base), those of segments III-VII thin, mesal paratergites broad; posterior middle of sternite VI with finely and densely setose patch, posterior edge of tergite VII with thin palisade fringe, median part of sternite VII as in Fig. 237, sternite VIII (Fig. 238), tergites IX (Fig. 239), X (Fig. 240) and aedeagus as in Figs 241-243, spermatheca as in Fig. 275.

Etymology - Named after the collector of most of the material, Harald Schillhammer (NHMW).

Distribution - Currently only known from Chin State in Myanmar and probably endemic to it.

Remarks - There is a labelling mistake in the type series, paratypes \#185 that were sifted should all be \#185a, leg. Aung Zaw Lin.

\section{Anotylus schuelkei sp. n.}

(Figs 84, 244-250, 276)

Type material - Holotype (ð): CHINA: Yunnan, Nujiang Lisu Auton. Prefecture, Gaol-

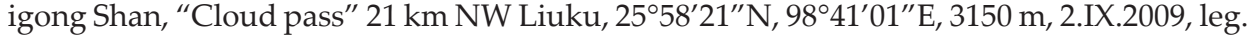
M. Schülke (CH09-22), shrubs \& bamboo, litter sifted (coll. Schülke in ZMHB); Paratypes (285): same data as holotype (24, coll. Schülke, 2, IZAS); CHINA: Yunnan, Nujiang Lisu

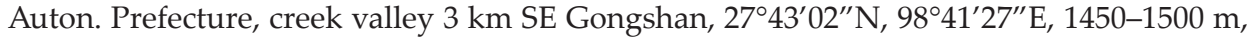
5.VI.2007, leg. D.W. Wrase (20), litter/moss sifted (3, coll. Schülke); Yunnan, Nujiang Lisu

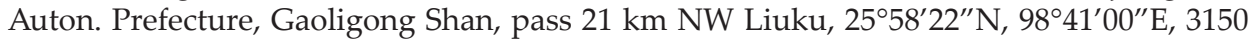
m, 9.VI.2007, leg. M. Schülke (CH07-26), bamboo with shrubs, litter sifted (1, coll. Schülke); Yunnan, Nujiang Lisu Auton. Prefecture, Gaoligong Shan, creek valley $20 \mathrm{~km} \mathrm{NW} \mathrm{Liuku,}$ 255' $49^{\prime \prime} \mathrm{N}, 9^{\circ} 41^{\prime} 48^{\prime \prime} \mathrm{E}, 3000 \mathrm{~m}, 9 . V I .2007$, leg. D.W. Wrase (27), bamboo, shrubs, litter sifted (11, coll. Schülke); Yunnan, Nujiang Lisu Auton. Prefecture, Gaoligong Shan, creek

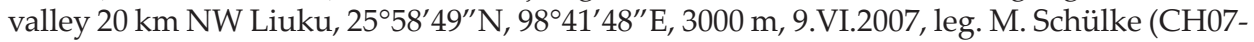
27), bamboo, shrubs, litter sifted (13, coll. Schülke); Yunnan, Nujiang Lisu Auton. Prefec-

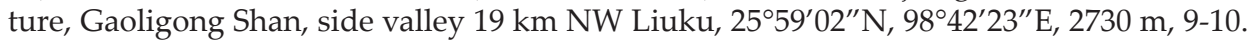
VI.2007, leg. D.W. Wrase (28), devastated primary forest, litter sifted (28, coll. Schülke, 1, SMNS, 1, CNCI, 1, SDEI, 1, FMNH, 1, ISNB, 1, MNHP, 1, NIBR, 1, BMNH, 1, NKME, 1,

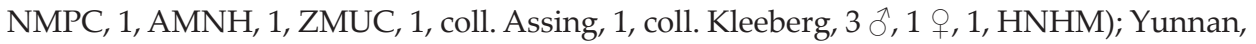
Nujiang Lisu Auton. Prefecture, Gaoligong Shan, side valley 19km NW Liuku, 2559'02”N, 98 42'23"E, 2730 m, 9.VI.2007, leg. M. Schülke (CH07-28), devastated primary forest, litter sifted (65, coll. Schülke); same but 10.VI.2007 (CH07-28a) (49, coll. Schülke); Yunnan, Nu-

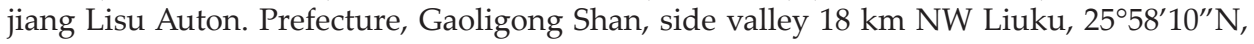
98 42'27"E, 2590 m, 9-10.VI.2007, leg. D.W. Wrase (29), devastated primary forest, litter sifted (6, coll. Schülke); Yunnan, Nujiang Lisu Auton. Prefecture, Gaoligong Shan, "Cloud pass" 21 km NW Liuku, 2558'21"N, 9841’01"E, 3150 m, 2.IX.2009, leg. D.W. Wrase (22a), shrubs, Vaccinium, bamboo, litter sifted (4, coll. Schülke, 6, MHNG); Yunnan, Nujiang Lisu Auton. Prefecture, Gaoligong Shan, "Cloud pass" $21 \mathrm{~km}$ NW Liuku, 2558'21"N, 98 41'01"E, 3150 m, 3.IX.2009, leg. M. Schülke (CH09-22a), shrubs and bamboo, litter sifted 
(10, coll. Schülke, 1, HNHM); Yunnan, Nujiang Lisu Auton. Prefecture, Gaoligong Shan, W “Cloud pass" 24 km NW Liuku, 2559'02”N, 98³9'56.5”E, 2940 m, 3.IX.2009, leg. M. Schülke (CH09-24), small cleft, wet moss \& litter sifted (1, coll. Schülke); Yunnan, Nujiang Lisu

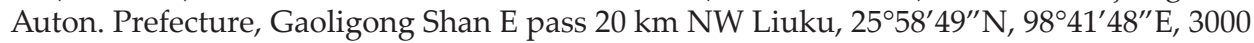
m, 3.IX.2009, leg. D.W. Wrase (25), creek valley with devastated primary forest, ferns, litter/ moss sifted (13, coll. Schülke, 2, IZAS, 7, NHMW); Yunnan, Nujiang Lisu Auton. Prefec-

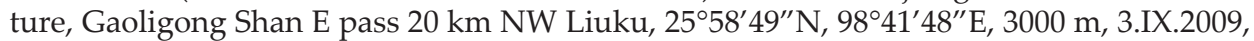
leg. M. Schülke (CH09-25), creek valley with devastated primary forest, litter \& moss sifted (20, coll. Schülke).

Description - Measurements (in mm, $\mathrm{n}=10)$ : $\mathrm{HW}=0.60(0.57-0.62) ; \mathrm{TW}=0.60(0.56-$ $0.63) ; \mathrm{PW}=0.79$ (0.73-0.81); $\mathrm{EW}=0.79(0.72-0.84) ; \mathrm{AW}=0.94(0.88-1.02) ; \mathrm{HL}=0.45(0.43-$ $0.47) ; \mathrm{EL}=0.10(0.10-0.11) ; \mathrm{TL}=0.18(0.17-0.18) ; \mathrm{PL}=0.48(0.46-0.50) ; \mathrm{SC}=0.33(0.31-0.35)$; $\mathrm{FB}=1.33(1.27-1.35) ; \mathrm{BL}=2.98$ (2.83-3.07). Habitus as in Fig. 84. Forebody moderately shining because of conspicuous light colouration and loose sculpture, abdomen very finely microsculptured with similar moderate lustre. Body somewhat yellowish light brown, abdominal bases darker, two blackish spots at the bases of supraantennal prominences near circular clypeal impression and two similar impressed dark pits at hind vertex. Head with anterior margin possessing thin rim, somewhat unevenly arched, anterior clypeal rim very slightly projecting in middle, supraantennal prominences as moderately elevated oblique ridges, disc impressed near eyes. Epistomal suture forming subcircular impression (inside almost unsculptured), upper surface without tactile setae, eyes small and slightly bulging; in dorsal view temples (1.8× length of eye) alatiform, laterally rounded and posteriorly constricted to form distinct neck, however, dorsal side not separated by occipital furrow, rather by dorsum of neck bearing microsculpture instead of punctation. Antennomere 1 flattened club-shaped and smooth, segment 2 ovoid, segment 3 club-shaped, segment 4 small and isodiametricalal, segments 5-11 with basal dishes, articles 6-10 slightly transverse (1.0-1.3× broader than long). Head and pronotum with mostly foveate sculpture. Pronotum with lateral portion explanate, edge with a few short setae, lateral margin almost evenly arched, hind margin before corners slightly concave; disc medially with a pair of shinier parallel longitudinal ridges, partly impressed alongside. Elytra with insignificant membranous posterior margin extending from sutural corner to $3 / 4$ of hind margin. Elytral epipleural ridge present with up-turned lateral alatiform portions, disc depressed; shoulders weakly developed, surface foveolate, in between punctures rather smooth, apical margin slightly oblique. Legs short, pro- and mesotibia with several spinulose rows, metatibia with longitudinal ctenidium of spinules in distal half. Abdomen with sides moderately arcuate, second segment with paratergites strongly broadening posteriorly (abdomen appearing constricted at base), those of segments III-VII thin, mesal paratergites broad; posterior edge of tergite VII with thin palisade fringe, median part of sternite VII as in Fig. 244, sternite VIII (Fig. 245), tergites IX (Fig. 246), X (Fig. 247) and aedeagus as in Figs 248-250, spermatheca as in Fig. 276.

Etymology - The species is named after the collector of most of the material, Michael Schülke (Berlin), a private collector whose collection now belongs to ZMHB.

Distribution - Known only from a small area in Yunnan province (China). Remarks - The presence of this species suggests that similar brachypterous species may exist elsewhere in the more mountainous areas of China. 


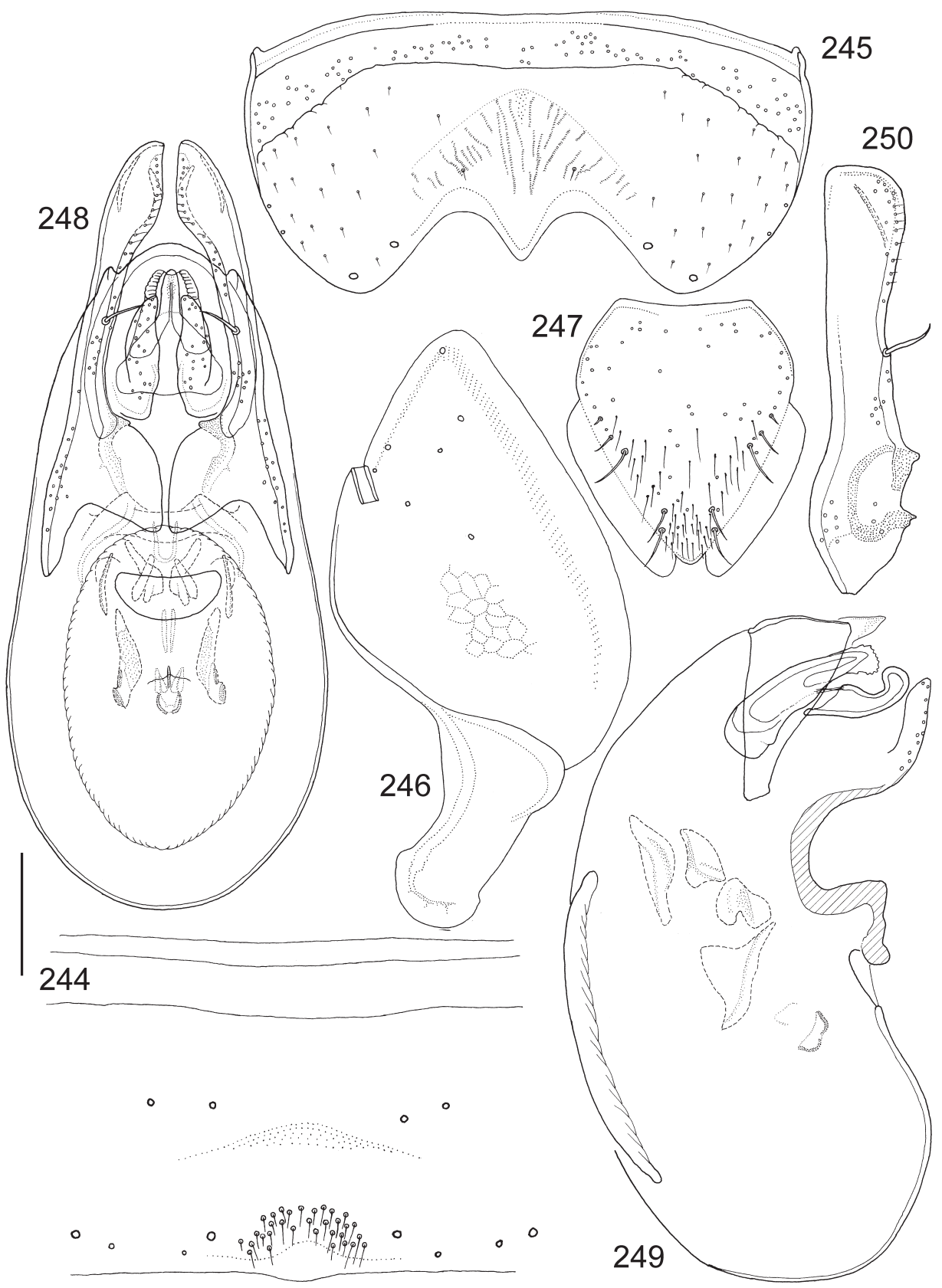

Figs 244-250. Anotylus schuelkei sp. n., male. $244=$ median part of sternite VII, $245=$ sternite VIII, 246 = tergite IX, 247 = tergite X, 248 = aedeagus, frontal view , $249=$ median lobe, lateral view, 250 = paramere, lateral view. Scales: $0.10 \mathrm{~mm}$ for Figs 244, 248-250, $0.12 \mathrm{~mm}$ for Figs 245-247 


\section{Anotylus tanator sp. n. \\ (Figs 97, 251-257, 277)}

Type material - Holotype (ふ̋): INDONESIA/SULAWESI: S-, Tanah Toraja, [Baruppu,] Pulu Pulu [0248.770'S, 119²7.616'E], 1700 m, 13-16.VIII.1990, leg. A. Riedel, sifted in forest (SMNS); Paratypes (25): same data as holotype (2 $\hat{\sigma}, 1$ q, SMNS, $1 \hat{\jmath}$, HNHM); Tanah Toraja, [Baruppu,] Ponding - Pulu Pulu [0249.550'S, 119²47.335'E], 1600-1800 m, 13-17.

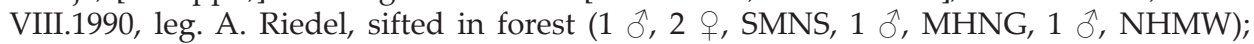
W-, Mamasa, Penanang, W of Mamasa, 1600 m [0254`38“S, 119¹8`30“E], 9.IV.1991, leg. D. Agosti (F91724) (16), montane rainforest, leaf litter (1, FMNH), same but (F91722) (2, MHNG); W-, Mamasa, Ulumambi, W of Mamasa, $1000 \mathrm{~m}$ [02 $\left.55^{\prime} 57^{\prime \prime} \mathrm{S}, 119^{\circ} 15^{\prime} 05^{\prime \prime} \mathrm{E}\right]$, 11.IV.1991, leg. D. Agosti (F91741) (17), remnants of rainforest / coffee plantation, leaf litter (3, MHNG, 1, AMNH); W-, Mamasa, Ulumambi, W of Mamasa, $1300 \mathrm{~m}\left[02^{\circ} 55^{`} 09^{\prime \prime} \mathrm{S}\right.$, $119^{\circ} 16^{\prime} 22^{\prime \prime} \mathrm{E}$ ], 12.IV.1991, leg. D. Agosti (18), montane forest, leaf litter (6, MHNG, 1, ISNB, 1, ZMUC, 1, MNHP).

Description - Measurements (in mm, $\mathrm{n}=10)$ : $\mathrm{HW}=0.70(0.64-0.74) ; \mathrm{TW}=0.69(0.62-$ $0.72) ; \mathrm{PW}=1.02$ (0.93-1.08); $\mathrm{EW}=1.10$ (1.05-1.16); $\mathrm{AW}=1.18(1.08-1.26) ; \mathrm{HL}=0.55$ (0.50$0.57) ; \mathrm{EL}=0.14(0.13-0.15) ; \mathrm{TL}=0.21(0.19-0.23) ; \mathrm{PL}=0.60(0.54-0.65) ; \mathrm{SC}=0.65(0.61-0.69)$; $\mathrm{FB}=1.88$ (1.75-1.95); $\mathrm{BL}=3.62$ (3.35-3.90). Habitus as in Fig. 97. Forebody weakly shining because of sculpture; abdomen with such extremely dense coriaceous microsculpture that not even traces of punctation observable, opaque. Forebody, legs and abdomen reddish medium to dark brown, except explanate part of pronotum and clypeal area (both for transparency), these, mouthparts and antennae reddish medium brown. Head with a thin rim on anterior margin, truncate in middle, laterally evenly arched towards eyes, supraantennal prominences as moderately elevated oblique ridges, disc slightly impressed near eyes. Epistomal suture forming subcircular impression (almost impunctate inside), upper surface without tactile setae, eyes moderately small, very slightly bulging from sideline of head; in dorsal view temples (1.5× length of eye) alatiform, laterally imperfectly rounded (without being angled) and posteriorly constricted to form distinct neck, not separated dorsally by occipital furrow, only by neck being microsculptured rather than punctured. Antennomere 1 club-shaped, finely sculptured, segment 2 elongate oval, segment 3 clubshaped, segment 4 small and isodiametrical, segments 5-11 with basal dishes, articles 6-10 transverse (about $1.5 \times$ broader than long), segments 9-10 bulkier than preceding. Head and pronotum with mostly lacunose sculpture. Pronotum with lateral portion explanate and slightly up-turned, edge alatiform with sparse short setae; lateral margin more or less evenly arched with a number of small, irregularly distributed denticles, posterior margin broadly incised before corners; disc medially with two parallel longitudinal ridges, almost confluent posteriorly, impressed alongside them, laterally impressed around middle of sides. Elytra with membranous posterior margin extending from sutural corner to 3/4 of hind margin. Elytral epipleural ridge present with up-turned lateral alatiform portions, disc longitudinally impressed alongside; shoulders well developed, surface foveolate, in between punctures torulose. Legs short, pro- and mesotibia with several spinulose rows, metatibia with longitudinal ctenidium of spinules in distal half. Abdomen with sides rather arcuate, second segment with paratergites strongly broadening posteriorly (abdomen appearing constricted at base), those of segments III-VII thin, mesal paratergites broad, posterior edge of tergite VII with thin palisade fringe, median part of sternite VII as in Fig. 251, sternite VIII (Fig. 252), tergites IX (Fig. 253), X (Fig. 254) and aedeagus as in Figs 255-257, spermatheca as in Fig. 277. 


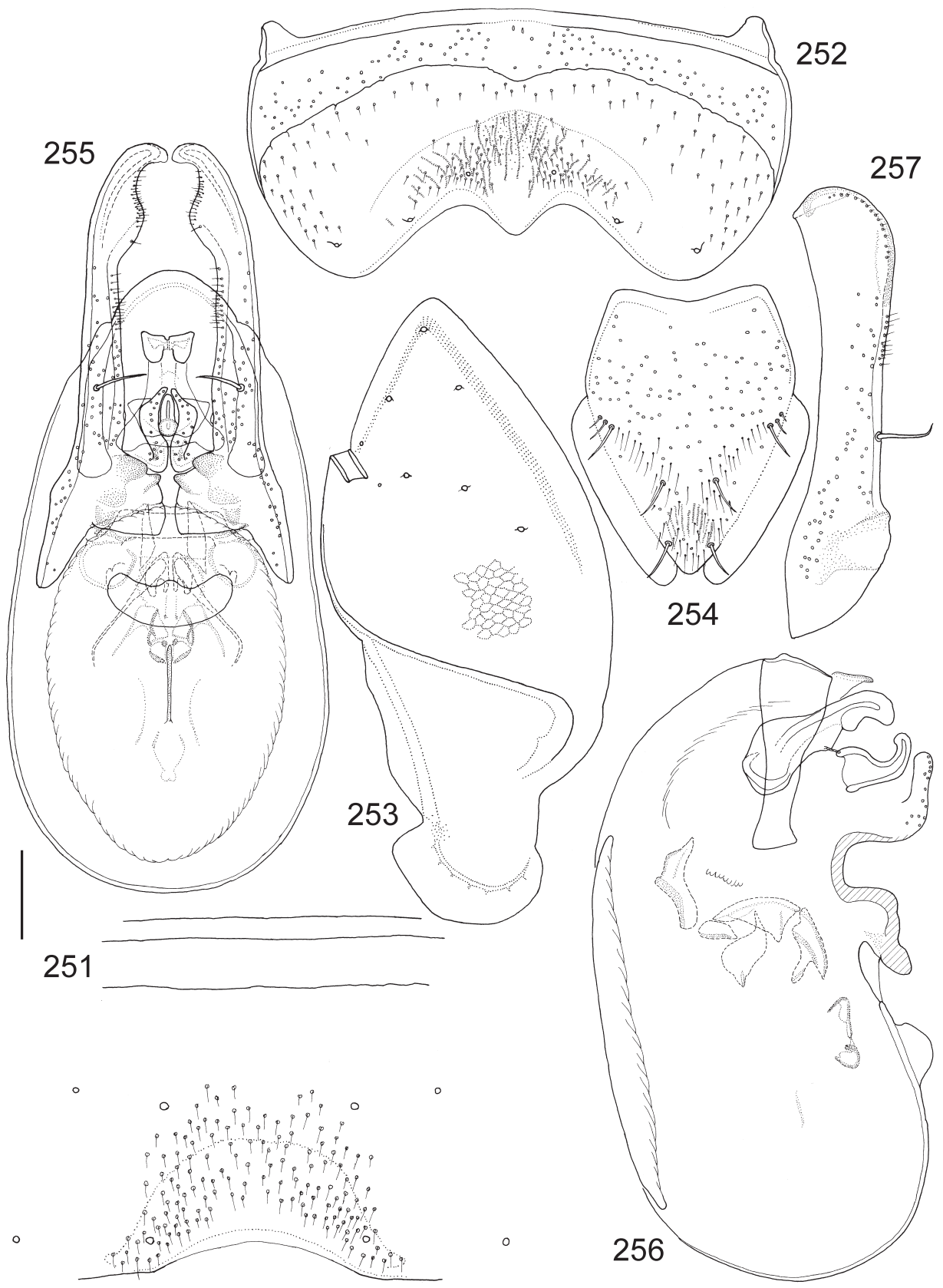

Figs 251-257. Anotylus tanator sp. n., male. $251=$ median part of sternite VII, $252=$ sternite VIII, 253 = tergite IX, 254 = tergite X, 255 = aedeagus, frontal view, $256=$ median lobe, lateral view, 257 = paramere, lateral view. Scales: $0.09 \mathrm{~mm}$ for Fig. 251, $0.10 \mathrm{~mm}$ for Figs 255-257, $0.11 \mathrm{~mm}$ for Figs 253-254, $0.13 \mathrm{~mm}$ for Fig. 252 
Etymology - The specific epithet is composed of the beginnings of the two words of the type locality area, Tanah Toraja (noun in apposition).

Distribution - According to administrative areas the species is known from an area partly belonging to W Sulawesi (Mamasa regency), partly $S$ Sulawesi (Tana Toraja regency).

Remarks - It is apparently related to A. rurukan (also from Sulawesi, but from the northern part).

\section{Anotylus topali sp. n. \\ (Figs 93, 258-264, 278)}

Type material - Holotype (đ): VIETNAM: Ninh binh, Cuc phuong [National Park], [2021'04"N, 105³5’34"E, ca. 400 m,] 12-18.V.1966, leg. Gy. Topál (no. 384), sifted litter of forest (HNHM); Paratypes (50): VIETNAM: Ninh binh, Cuc phuong, 5-18.V.1966, leg. Gy. Topál (no. 385), from 25 trap of soil in forest near house (1 §, 3, HNHM, 1, MNHP, 1 , BMNH, 1, SMNS, 1, MHNG, 1, ISNB); Ninh binh, Cuc phuong, 6-18.V.1966, leg. Gy. Topál (no. 386), from 12 trap of soil in dry forest (1 ․ HNHM); Cuc Phuong NP, $20^{\circ} 15.586^{\prime} \mathrm{N}$, $105^{\circ} 42.320^{\prime} \mathrm{E}, 147$ m, 30.IV.-1.V.2005, leg. A. Kun (1, HNHM), Quang Binh pr., W of Chalo, westerly stream, $17^{\circ} 43^{\prime} \mathrm{N}, 105^{\circ} 46^{\prime} \mathrm{E}, 17 . \mathrm{IV} .1998$, leg. L. Herman, leaf litter near stream $(2$, AMNH); Ha Tinh pr., Raò An, 13 km W Huang Son, rt. 8, $7 \mathrm{~km}$ S Nuòc Sôt village, $18^{\circ} 21^{`} \mathrm{~N}$, $105^{\circ} 15^{`} \mathrm{E}, 300 \mathrm{~m}, 9-22 . V .1998$, leg. L. Herman, flight intercept trap (3, AMNH); Ha Tinh pr., Raò An, $13 \mathrm{~km}$ W Huang Son, rt. 8, 7 km S Nuòc Sôt village, $18^{\circ} 21^{`} \mathrm{~N}, 105^{\circ} 15^{`} \mathrm{E}, 220 \mathrm{~m}$, 22.IV.1998, leg. L. Herman, mushrooms (2, AMNH); Ha Tinh pr., Raò An, 13km W Huang Son, rt. 8, 7 km S Nuòc Sôt village, $18^{\circ} 21^{`} \mathrm{~N}, 105^{\circ} 15^{`} \mathrm{E}, 220 \mathrm{~m}, 26 . \mathrm{IV} .1998$, leg. L. Herman, litter near stream (6, AMNH), same but $250 \mathrm{~m}$, 27.IV.1998, seep near river (2, AMNH), same but 29.IV.1998, (2967-2968), litter near seep (2, AMNH); Ha Tinh pr., Raò An, 13 km W Huang Son, rt. 8, 7 km S Nuòc Sôt village, $18^{\circ} 21^{`} \mathrm{~N}, 105^{\circ} 15^{`} \mathrm{E}, 270 \mathrm{~m}$, 30.IV.1998, leg. L. Herman, litter near forest stream $(1 \stackrel{+}{\uparrow}, \mathrm{AMNH}, 1 \hat{\jmath}, \mathrm{ZMUC}, 1 \hat{\jmath}, \mathrm{FMNH}, 1 \hat{\jmath}, \mathrm{NIBR}, 1 \hat{\jmath}$, CNCI, 1 §̊, IZAS), same but 300-350 m, 1.V.1998 (2, AMNH), same but $350 \mathrm{~m}, 1 . \mathrm{V} .1998$, litter near drying forest stream (3, AMNH); Ha Tinh pr., Raò An, 13 km W Huang Son, rt. 8, 7 km S Nuòc Sôt village, $18^{\circ} 21^{`} \mathrm{~N}, 1^{\circ} 5^{\circ} 15^{`} \mathrm{E}, 220$ m, 6.V.1998, leg. L. Herman, moist leaves near \& above stream (2, AMNH), same but 6.V.1998 (2983), leaves along stony shore (1, AMNH), same but 300 m, 7.V.1998 (2986), litter at stream overflow (2, AMNH), same but 13.V.1998 (2994-2995), leaf litter near stream (2, AMNH); Ha Tinh pr., Raò An, 13 km W Huang Son, rt. 8, 7 km S Nuòc Sôt village, $18^{\circ} 21^{`} \mathrm{~N}, 105^{\circ} 15^{`} \mathrm{E}, 280 \mathrm{~m}, 21 . \mathrm{V} .1998$, leg. L. Herman, litter above flood line of stream (2, AMNH); CHINA: Hubei, E Chongqing, Shiruguan, pass $5 \mathrm{~km}$ NW Taiyanghe, $30.65^{\circ} \mathrm{N}, 109.50^{\circ} \mathrm{E}$, ca. $1700 \mathrm{~m}, 1-9 . \mathrm{VII} .2003$, leg. J. Turna, pitfall traps (1 $\delta$, NHMW); Jiangxi prov., Jinggangshan Mts., Xiangzhou (forested valley S of the village), $26^{\circ} 35.5^{\prime} \mathrm{N}, 114^{\circ} 16.0^{\prime} \mathrm{E}, 374 \mathrm{~m}, 26 . \mathrm{IV} .2011$, leg. M. Fikaček \& J. Hájek (MF08), accumulation of moist leaf litter along the stream and on the steep slope above the stream in the sparse secondary forest, sifting ( 1 , NMPC); Yunnan, Mengla, Wangtianshu Scenic Area, $21^{\circ} 37^{\prime} 39.4^{\prime \prime} \mathrm{N}, 101^{\circ} 35^{\prime} 16.2^{\prime \prime} \mathrm{E}, 717 \mathrm{~m}$, 27.IX.2012, leg. D. Rédei (2), sifted from litter under banana trees $(1 \AA$, HNHM).

Description - Measurements (in mm, $\mathrm{n}=10)$ : $\mathrm{HW}=0.64(0.61-0.68) ; \mathrm{TW}=0.60(0.56-$ 0.65); $\mathrm{PW}=0.91$ (0.85-0.99); $\mathrm{EW}=0.98$ (0.94-1.02); $\mathrm{AW}=0.99$ (0.92-1.07); $\mathrm{HL}=0.51$ (0.49- 


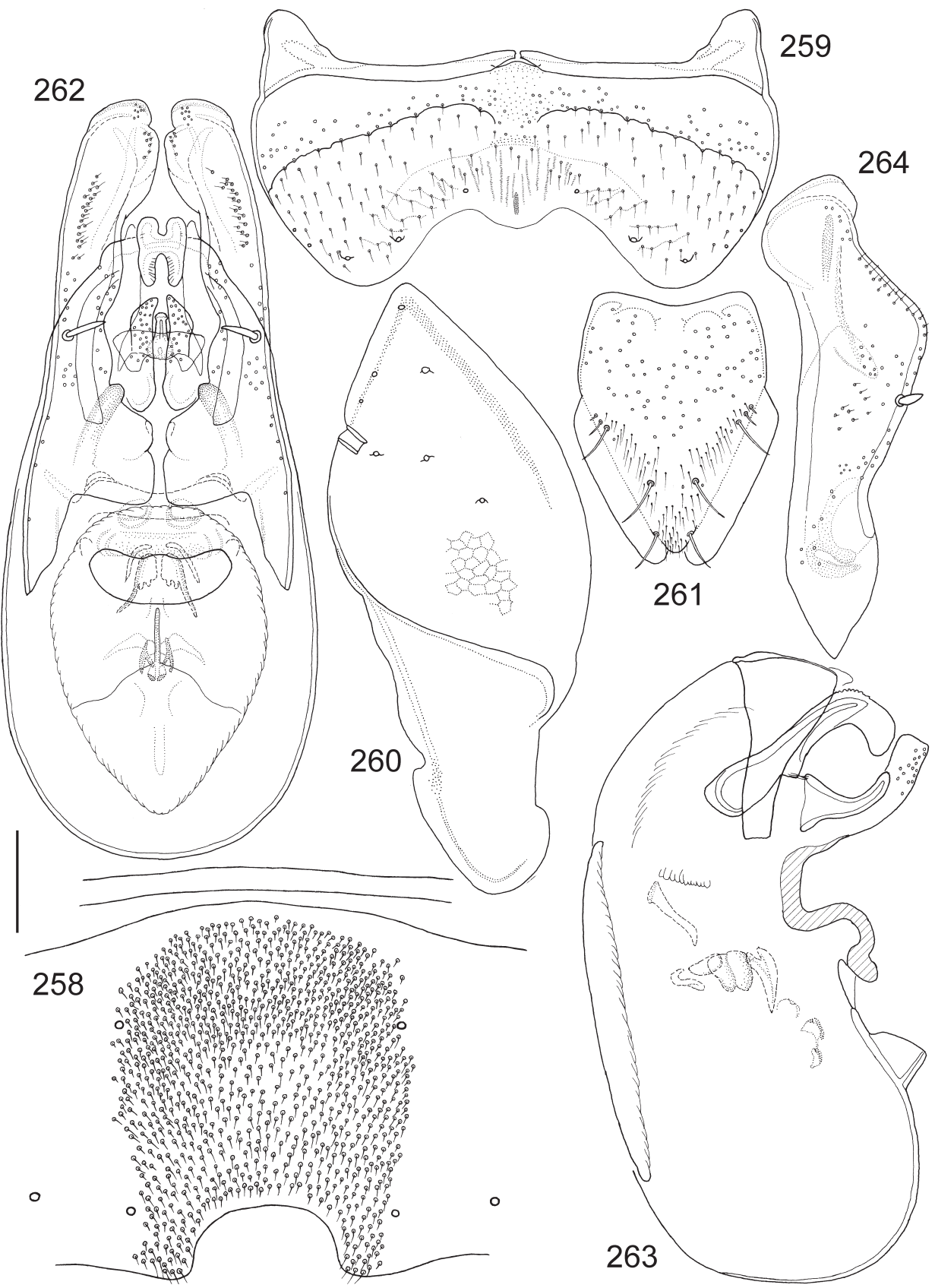

Figs 258-264. Anotylus topali sp. n., male. $258=$ median part of sternite VII, $259=$ sternite VIII, 260 = tergite IX, 261 = tergite X, 262 = aedeagus, frontal view , $263=$ median lobe, lateral view, 264 = paramere, lateral view. Scales: $0.085 \mathrm{~mm}$ for Fig. 258, $0.10 \mathrm{~mm}$ for Figs 262-264, $0.13 \mathrm{~mm}$ for Fig. 261, $0.145 \mathrm{~mm}$ for Fig. 260, $0.17 \mathrm{~mm}$ for Fig. 259 
$0.55) ; \mathrm{EL}=0.15(0.14-0.16) ; \mathrm{TL}=0.18(0.17-0.20) ; \mathrm{PL}=0.54(0.51-0.58) ; \mathrm{SC}=0.61(0.58-0.65)$; $\mathrm{FB}=1.71(1.65-1.80) ; \mathrm{BL}=3.26$ (3.06-3.48). Habitus as in Fig. 93. Forebody moderately shining despite sculpture, abdomen with very fine and dense microsculpture obscuring moderately large but extremly superficial punctures, surface with greasy lustre. Forebody reddish dark brown, clypeal area and explanate part of pronotum indistinctly lighter, mouthparts, legs, antennae and abdomen reddish medium to dark brown. Head with an extremely thin rim on anterior margin, latter truncate in middle, very slightly angled also at supraantennal prominences, these as moderately elevated oblique ridges, disc slightly impressed near eyes. Epistomal suture forming subcircular impression (almost unpunctured inside), upper surface without tactile setae, eyes moderately small and very slightly bulging from sideline of head; in dorsal view temples $(1.2 \times$ length of eye) alatiform, laterally rounded and posteriorly constricted to form distinct neck, not separated dorsally by occipital furrow, only by neck being microsculptured rather than punctured. Antennomere 1 spoon-shaped and lightly microsculptured, segment 2 with broad base and narrowing

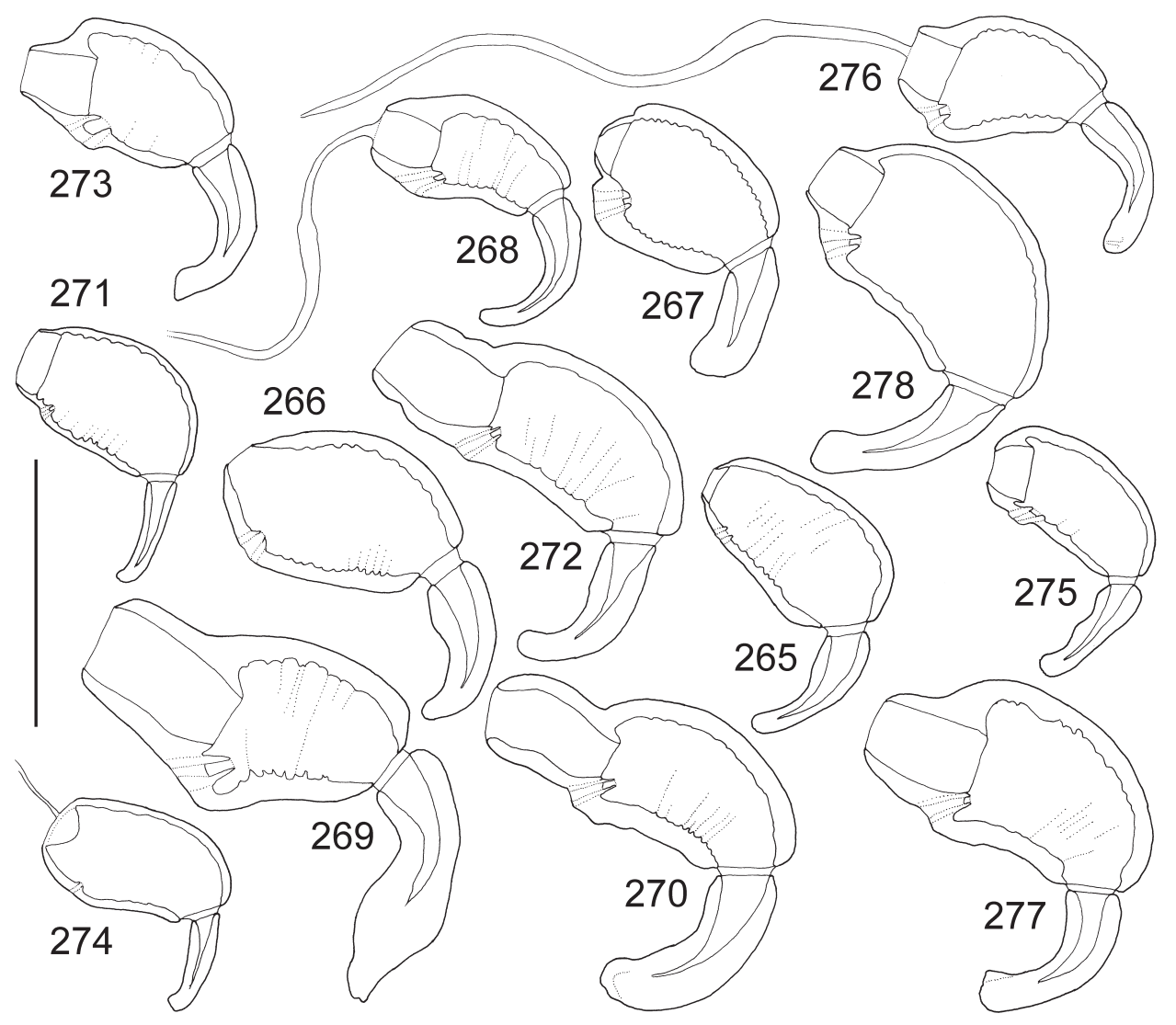

Figs 265-278. Spermathecae of Anotylus species: $265=$ A. coonoor sp. n., $266=$ A. ganapat sp. n., $267=A$. hartmanni sp. n., $268=A$. ijen sp. n., $269=A$. jambi sp. n., $270=A$. kabasi sp. n., $271=$ A. linaxi sp. n., $272=A$. riedeli sp. n., $273=$ A. rurukan sp. n., $274=A$. schawalleri sp. n., $275=$ A. schillhammeri sp. n., $276=$ A. schuelkei sp. n., $277=$ A. tanator sp. $\mathrm{n}, 278=$ A. topali sp. n. Scale: $0.10 \mathrm{~mm}$ 
apically, segment 3 stout club-shaped, segment 4 transverse discoid, segments 5-11 with basal dishes, transverse except last (segments $6-8$ almost $2 \times$ broader than long, while articles 9-10 less than 1.5× broader than long). Head and pronotum with mostly alveolate sculpture. Pronotum with lateral portion explanate and slightly up-turned, edge alatiform with sparse short setae; lateral margin unevenly arched with a number of small, irregularly distributed denticles, posterior corners rather shallowly incised; disc medially with two posteriorly nearly confluent longitudinal ridges, impressed alongside them, laterally strongly impressed around middle of sides. Elytra with membranous posterior margin extending from sutural corner to 3/4 of hind margin. Elytral epipleural ridge present with upturned lateral alatiform portions, disc longitudinally impressed alongside; shoulders well developed, surface foveolate, in between punctures torulose. Legs short, pro- and mesotibia with several spinulose rows, metatibia with longitudinal ctenidium of spinules in distal half. Abdomen with sides moderately arcuate, second segment with paratergites strongly broadening posteriorly (abdomen appearing constricted at base), those of segments III-VII thin, mesal paratergites broad; posterior edge of tergite VII with thin palisade fringe, median part of sternite VII as in Fig. 258, sternite VIII (Fig. 259), tergites IX (Fig. 260), X (Fig. 261) and aedeagus as in Figs 262-264, spermatheca as in Fig. 278.

Etymology - The species is named after the collector of the holotype, the recently deceased Dr. György Topál (1931-2016).

\section{Distribution - The species is currently known from southern China and Vietnam. \\ Remarks - The Hubei record is suspicious, raising doubt about the cor- rectness of the label (especially considering the rather battered condition of the sole specimen).}

Acknowledgements - The author would like to express thanks to the collectors who provided a lot of additional information, especially A. Riedel (Karlsruhe, Germany), A. Smetana (Ottawa, Canada) and J. Schmidt (Admannshagen, Germany). The manuscript was checked very carefully by Liang Lü (Beijing, China) and Harald Schillhammer (Wien, Austria), latter should also be thanked for the colour habitus photography done on many days in the course of about two years. Finally, Giulio Cuccodoro arranged several visits of the author at MHNG (2012, 2015, 2016), Wolfgang Schawaller one at SMNS (2013), these collections provided the bulk of the examined material.

\section{REFERENCES}

Bernhauer, M. (1926): Fauna Buruana. Zur Staphylinidenfauna der Molukken. - Treubia 7(3): 311-327.

Bernhauer, M. (1934): Siebenter Beitrag zur Staphylinidenfauna Chinas. - Entomologisches Nachrichtenblatt (Troppau) 8(1): 1-20.

Bernhauer, M. (1938): Zur Staphylinidenfauna von China u. Japan (9. Beitrag). - Entomologisches Nachrichtenblatt (Troppau) 12(1): 17-39. 
Biswas, D. N. \& Sengupta, T. (1980): Indian species of Oxytelopsis (Coleoptera: Staphylinidae: Oxytelinae). - Oriental Insects 14(3): 391-395. https://doi.org/10.1080/00305316. 1980.10434819

Cameron, M. (1925): New Staphylinidae from the Dutch East Indies. - Treubia 6(2): 174-198.

Cameron, M. (1928): Staphylinidae from Mt. Poi \& Mt. Penrissen, with descriptions of new species. - Sarawak Museum Journal 3(IV)(11): 399-412.

Cameron, M. (1930): The fauna of British India including Ceylon and Burma. Coleoptera. Staphylinidae. Vol. I. - Taylor and Francis, London, i-xvii + 471 pp.

Cameron, M. (1933): Staphylinidae (Col.) from Mount Kinabalu. - Journal of the Federated Malay States Museums 17(2): 338-360.

Cameron, M. (1934): Nouveaux Staphylinidae orientaux. - Bulletin de la Société Entomologique de France 39(2): 31-32.

Cameron, M. (1936): Fauna Javanica. The Staphylinidae (Col.) collected by Mr. F. C. Drescher. - Tijdschrift voor Entomologie 79(1-2): 25-54.

Coiffait, H. (1982): Contribution à la connaissance des Staphylinides de l'Himalaya (Népal, Ladakh, Cachemire) (Insecta: Coleoptera: Staphylinidae). - Senckenbergiana Biologica 62: 21-179.

Coiffait, H. (1984): Staphylinides (Col.) de la région himalayenne et de l'Inde. II. Tachyporinae, Oxytelinae et Aleocharinae. - Entomologica Basiliensia 9: 116-157.

Coiffait, H. (1986): Liste des exemplaires typiques de la collection Coiffait déposée au Muséum d'Histoire Naturelle de Paris. - Bulletin de la Société d'Histoire naturelle de Toulouse 125: 127-142.

Fauvel, A. (1895): Staphylinides nouveaux de l'Inde et de la Malaisie. - Revue d'Entomologie (Caen) 14: 180-286.

Fauvel, A. (1904): Staphylinides exotiques nouveaux. 2e Partie. - Revue d'Entomologie (Caen) 23: 76-112.

Hammond, P. M. (1975): Coleoptera: Staphylinidae Oxytelini from Ceylon. Report No. 34 from the Lund University Ceylon Expedition in 1962. - Entomologica Scandinavica Supplementum 4(1973-75): 141-178.

Hammond, P. M. (1976a): A review of the genus Anotylus C. G. Thomson (Coleoptera: Staphylinidae). - Bulletin of the British Museum (Natural History), Entomology 33(2): 137-187.

Hammond, P. M. (1976b): The crassicornis species-group of the genus Anotylus (Staphylinidae). - Systematic Entomology 1: 19-51. https://doi.org/10.1111/j.1365-3113.1976. tb00029.x

Hammond, P. M., Morgan, A., Morgan, A. V. (1979): On the gibbulus group of Anotylus, and fossil occurrences of Anotylus gibbulus (Staphylinidae). - Systematic Entomology 4: 215-221. https://doi.org/10.1111/j.1365-3113.1979.tb00637.x

Hammond, P. M. (1984): An annotated check-list of Staphylinidae (Insecta: Coleoptera) recorded from Borneo. - The Sarawak Museum Journal 33(54): 187-218.

Herman, L. H. (1970): Phylogeny and reclassification of the genera of the rove-beetle subfamily Oxytelinae of the World (Coleoptera, Staphylinidae). - Bulletin of the American Museum of Natural History 142(5): 343-454.

Herman, L. H. (2001): Catalog of the Staphylinidae (Insecta: Coleoptera). 1785 to the end of the second Millennium. - Bulletin of the American Museum of Natural History 265: 1-4218. https://doi.org/10.1206/0003-0090(2001)264<0003:NCITSI>2.0.CO;2

Iто, T. (1987): Two new species of the genus Oxytelopsis from Japan and Taiwan (Coleoptera, Staphylinidae). - The Entomological Review of Japan 42(Supplement): 75-79. 
Iто, T. (2007): Additional Distribution of Oxytelopsis shibatai T. Ito (Coleoptera: Staphylinidae). - The Entomological Review of Japan 42(1): 173-174.

Makranczy, GY. (2006): Systematics and phylogenetic relationships of the genera in the Carpelimus group (Coleoptera: Staphylinidae: Oxytelinae). - Annales historico-naturales Musei nationalis hungarici 98: 29-119.

Makranczy, Gy. (2011): Four new Neotropical species of Anotylus with an interesting sexual dimorphism (Coleoptera, Staphylinidae: Oxytelinae). - Annales historico-naturales Musei nationalis hungarici 103: 43-64.

Makranczy, Gy. (2015): New nomenclatural and taxonomic acts, and comments: Staphylinidae: Oxytelinae. P. 22. In: Löbl, I. \& Löbl, D. (eds): Catalogue of Palaearctic Coleoptera. Volume 2/1. Hydrophiloidea - Staphylinoidea. Revised and updated edition. Volume 1. Brill, Leiden and Boston, i-xxv, 900 pp.

Makranczy, Gy. (2016): Review of the genus Paraploderus Herman, 1970 (Coleoptera: Staphylinidae: Oxytelinae). - Acta Zoologica Academiae Scientiarum Hungaricae 62(1): 59-116. https://doi.org/10.17109/AZH.62.1.59.2016

Paulian, R. (1940): Un nouveau Coléoptère staphylinide des Indes Anglaises. - Bulletin de la Société Entomologique de France 45: 57-59.

Paulian, R. (1941): Les premiers états des Staphylinoides (Coleoptera) étude de morphologie comparée. - Mémoires du Muséum National d'Histoire Naturelle (n. sér.) 15: 1-361.

ScheERpeltz, O. (1965): Wissenschaftliche Ergebnisse der Schwedischen Expedition 1934 nach Indien und Burma. Coleoptera Staphylinidae (except. Megalopsidiinae et Steninae). (5. Beitrag zur Kenntnis der orientalischen Staphyliniden). - Arkiv för Zoologi (2)17(2): 93-371.

Ubaidillah, R. (2002): A list of the recent insect type-specimens in the Museum Zoologicum Bogoriense. - Research Center for Biology - LIPI, Bogor, 204 pp.

Received July 17, 2016, accepted September 17, 2016, published June 19, 2017 